ABSTRACT

$\begin{array}{ll}\text { Title of dissertation: } & \text { MEASUREMENT OF THE W BOSON } \\ & \text { MASS AND WIDTH USING A NOVEL } \\ & \text { RECOIL MODEL }\end{array}$

Matthew J Wetstein

Doctor of Philosophy, 2009

Dissertation directed by: Professor Sarah C. Eno

Department of Physics

This dissertation presents a direct measurement of the $W$ boson mass $\left(M_{W}\right)$ and decay width $\left(\Gamma_{W}\right)$ in $1 \mathrm{fb}^{-1}$ of $W \rightarrow e \nu$ collider data at $\mathrm{D} \varnothing$ using a novel method to model the hadronic recoil. The mass is extracted from fits to the transverse mass $M_{\mathrm{T}}, p_{\mathrm{T}}(e)$, and $E_{T}$ distributions. The width is extracted from fits to the tail of the $M_{\mathrm{T}}$ distribution. The electron energy measurement is simulated using a parameterized model, and the recoil is modeled using a new technique by which $\mathrm{Z}$ recoils are chosen from a data library to match the $p_{\mathrm{T}}$ and direction of each generated $\mathrm{W}$ boson. We measure the the $W$ boson mass to be $M_{W}=80.4035 \pm 0.024($ stat $) \pm 0.039($ syst $)$ from the $M_{\mathrm{T}}, M_{W}=$ $80.4165 \pm 0.027($ stat $) \pm 0.038($ syst $)$ from the $p_{\mathrm{T}}(e)$, and $M_{W}=80.4025 \pm 0.023($ stat $) \pm 0.043($ syst $)$ from the $E_{T}$ distributions. $\Gamma_{W}$ is measured to be $\Gamma_{\mathrm{W}}=2.025 \pm 0.038($ stat $) \pm 0.061$ (syst) GeV. 


\title{
MEASUREMENT OF THE W BOSON MASS AND WIDTH USING A NOVEL RECOIL MODEL
}

\author{
by \\ Matthew J Wetstein \\ Dissertation submitted to the Faculty of the Graduate School of the \\ University of Maryland, College Park in partial fulfillment \\ of the requirements for the degree of \\ Doctor of Philosophy \\ 2009
}

Advisory Committee:

Professor Sarah C. Eno, Chair/Advisor

Professor Nick Hadley

Professor Hassan Jawahery

Associate Professor Kaustaub Agashe

Professor William McDonough 


\section{Dedication}

B"H. To my family, friends, and teachers. 


\section{Acknowledgments}

To start, I would like to thank my parents for supporting and encouraging my interest in science from the beginning. I would not have come this far without them. Thanks to Jonathan for being a great brother, for his genuine curiosity about my work, and for putting up with all the times I lectured him on science when we were kids. My fiance Karen was amazing through these challenging years in my career, counseling me throughout, and giving me her utmost excitement and enthusiasm.

Thank you to all of my amazing teachers in the Parsippany School system for giving me the necessary skills to succeed in life. I am also truly grateful to the faculty of the Rutgers Physics department for the fantastic undergraduate education I received. The physics department at University of Maryland also has my gratitude for challenging me and for nurturing my growth into a mature scientist. I am indebted to the staff and scientists at Fermilab and DØ for making the $\mathrm{W}$ mass and width measurements possible, and for providing me with an unparalleled scientific environment to grow in. Thanks also to my friends at the Mercury Cafe for letting me use their space as my second office throughout the writing of this dissertation, and for providing me with ample, high-quality caffeinated beverages.

I want to thank Sally Megonigal and Jane Hessing for their grace, kindness, and patience in helping me navigate the University of Maryland graduate program throughout these 8 years. I could not have survived without them.

I would like to acknowledge Dick Kellog, who provided me with my first hands-on graduate experience in HEP, working on the test-beam at CERN. I still fondly recall my first day when he walked me through the trigger electronics with an oscilloscope.

To my University of Maryland Professors, colleagues and friends: I would like to express my appreciation to the Professors Nick Hadley, Andris Skuja, and Drew Baden for their mentorship and leadership in the Maryland HEP group. Many thanks also to Terry Toole, Michiel Sanders, Lei Wang, and Ming Yang for their company and guidance during my tenure at Fermilab. I want 
to thank Chad Jarvis for his friendship along the journey from College Park, Maryland to Batavia. Lastly, I would like to acknowledge Marco Verzocci, office-mate and teacher, who pushed me hard on my first year with DØ and prepared me for the intense work that would follow.

I would like to thank my friends and colleagues among the entire $\mathrm{W}$ mass group: Tim Andeen, Mikolaj Cwiok, Sarah Eno, Martin Grunewald, Feng Guo, Jun Guo, Mike Hildreth, John Hobbs, Bob McCarthy, Alex Melnitchouk, Jyotsna Osta, Pierre Petroff, Michael Rijssenbeek, Heidi Schellman, Jan Stark, Sahal Yacoob and Junjie Zhu. This measurement was a team effort and we could not have done it without the collaboration of the whole group. Junjie Zhu has served at least five different roles as my predecessor, colleague, convener, teacher, and friend.

I want to thank our W mass conveners, Pierre Petroff and Jan Stark for their tireless work and dedication. Jan deserves special mention as my most significant teacher at Fermilab. I owe him a debt of gratitude for his insightful instruction in precision physics.

Finally, I would not have had the opportunity to realize my full potential without the mentorship, guidance, and council of my advisor, Sarah Eno. I am forever grateful to her for pushing me to work hard and stay on target, and for her invaluable physics insights and career advice. I could not have hoped for a better advisor. 


\section{TABLE OF CONTENTS}

List of Figures viii

List of Abbreviations $\quad$ xiv

1 Introduction 1

2 Review of Theoretical Particle Physics 3

2.1 The Standard Model . . . . . . . . . . . . . . . . . . . . . . 3

2.2 Production and Decay of the $\mathrm{W}$ and $\mathrm{Z}$ Boson $\ldots \ldots \ldots \ldots$. . . . . . . 7

2.3 The $W$ boson Mass and Width . . . . . . . . . . . . . . . . . . . 11

3 Measurement Strategy 16

3.1 Kinematics and Observables . . . . . . . . . . . . . . . . . . . 16

3.2 Template Production . . . . . . . . . . . . . . . . . . . . . . 20

3.2 .1 Generator . . . . . . . . . . . . . . . . . . 20

3.2 .2 Detector Simulation . . . . . . . . . . . . . . . . . . . 21

3.2 .3 MC Testing . . . . . . . . . . . . . . . . . . . . . . 21

3.3 Basic Fit Strategy . . . . . . . . . . . . . . . . . . . . . . . 22

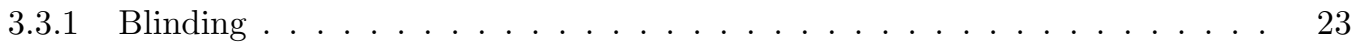

4 Experimental Setup 24

4.1 The FermiLab Accelerator . . . . . . . . . . . . . . . . . . . . . . . . . . 24

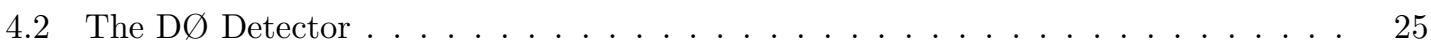

4.2 .1 Tracking System . . . . . . . . . . . . . . . . . . . 26

4.2.2 The Silicon Microstrip Tracker . . . . . . . . . . . . . . . . . . . . 27

4.2 .3 The Central Fiber Tracker . . . . . . . . . . . . . . . . . . . . . . 30

4.2 .4 Preshower Detectors . . . . . . . . . . . . . . . . . . . . . . . . . 31

4.2 .5 Calorimeter . . . . . . . . . . . . . . . . . . . . . 31

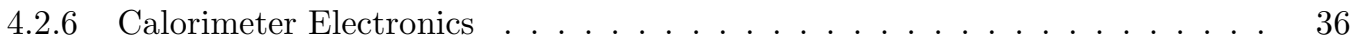

4.2 .7 Muon System . . . . . . . . . . . . . . . . . . . . . . 36

4.2 .8 Luminosity Counters . . . . . . . . . . . . . . . . . . . . 38

5 Optimizing the RunII Detector 39

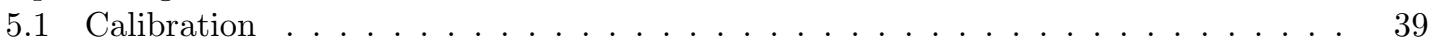

5.1.1 Intercalibration In $\phi$ Using Generic Collider Data . . . . . . . . . . . . . . 41

5.1.2 Absolute Calibration of Rings in $\eta$ Using $Z \rightarrow e e$ data . . . . . . . . . . . . 42

5.1 .3 Result of Calibration . . . . . . . . . . . . . . . . . . . 44

5.2 Energy Loss Corrections . . . . . . . . . . . . . . . . . . . . . 47

5.3 Material Studies . . . . . . . . . . . . . . . . . . . . 47

6 Event Selection 59

6.1 Data . . . . . . . . . . . . . . . . . . . . . . 59

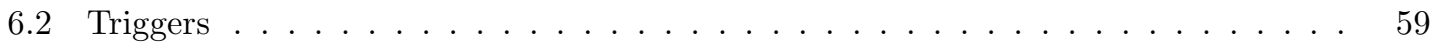

6.3 Track and Vertex Reconstruction . . . . . . . . . . . . . . . . . . . . . . 60

6.4 EM Reconstruction and Clustering Algorithm . . . . . . . . . . . . . . . . 60

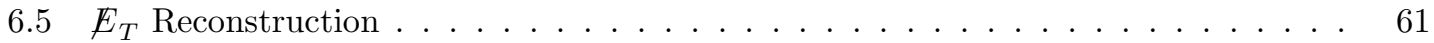

6.6 Particle Identification $\ldots \ldots \ldots \ldots$

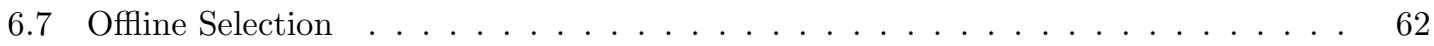


7 Electron Measurement and Simulation 65

7.1 Electron Energy Measurement . . . . . . . . . . . . . . . . . . . . . 65

7.1.1 Parameterized Energy Response . . . . . . . . . . . . . . . . . . 65

7.1 .2 Parameterized Energy Resolution . . . . . . . . . . . . . . . . . . 66

7.1.3 Underlying Energy Corrections . . . . . . . . . . . . . . . . . . . . 69

7.2 Electron Position Resolution . . . . . . . . . . . . . . . . . . . . . . . . 70

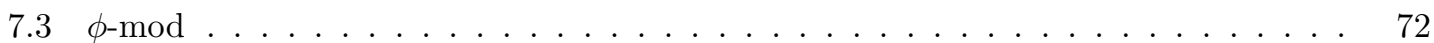

7.4 Final State Photon Merging . . . . . . . . . . . . . . . . . . . . 72

7.5 Primary Vertex Simulation _. . . . . . . . . . . . . . . 76

7.6 Luminosity and Run Number Simulation . . . . . . . . . . . . . . . 76

8 Efficiencies 79

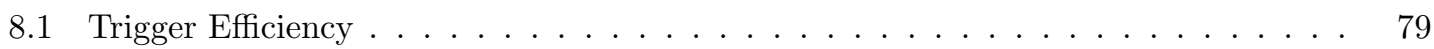

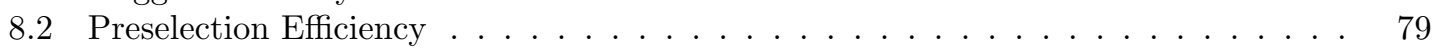

8.3 Track Matching Efficiency . . . . . . . . . . . . . . . . . . . . . . 81

8.4 EMID (Hmatrix) Efficiency . . . . . . . . . . . . . . . . . . . . . 81

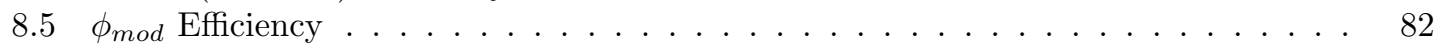

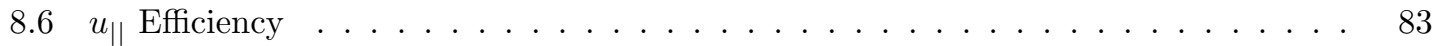

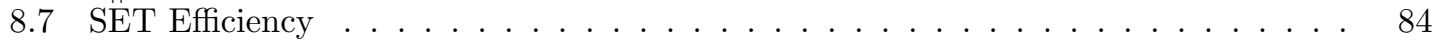

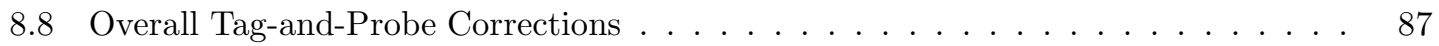

9 Recoil Measurement and Simulation $\quad 89$

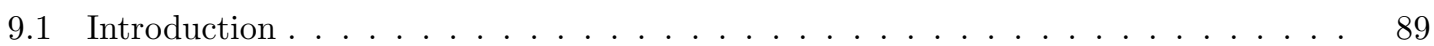

9.2 Recoil Library Method . . . . . . . . . . . . . . . . . . . . . . . . . . . . 90

9.2 .1 Overview . . . . . . . . . . . . . . . . . . . . 90

9.2 .2 Preparing the Recoil Library _ . . . . . . . . . . . . . . . . . . . 90

9.2 .3 Removing The Two Electrons from $Z$ Decays . . . . . . . . . . . . . . 90

9.2.4 Minimizing the Effects of FSR Photons . . . . . . . . . . . . . . . . 91

9.2.5 Correcting for Electron Selection Efficiencies _ . . . . . . . . . . . . 92

9.3 Bayseian Unfolding . . . . . . . . . . . . . . . . . . . . . . . . . . . 92

9.3 .1 Unfolding Method . . . . . . . . . . . . . . . . . . . . . . 92

9.3.2 Multidimensional Unfolding Using Bayes Theorem . . . . . . . . . . . . . . 93

9.3.3 Unfolding the Recoil Distribution . . . . . . . . . . . . . . . . . . . . . 94

9.4 A Brief Description of the Parameterized Recoil Model . . . . . . . . . . . . . . . 99

9.5 Biases and Uncertainties Particular to the Recoil Library Method . . . . . . . . . . 99

9.5.1 Fast MC Closure and Limited Statistical Power of the $Z$ Recoil Sample . . 99

9.5.2 Systematic Effects . . . . . . . . . . . . . . . . . . . . . . . . . . 101

9.5 .3 Unmerged FSR Photons . . . . . . . . . . . . . . . . . . . . . 103

9.5.4 Differences in Geometric Acceptance . . . . . . . . . . . . . . . . . . . 103

9.5.5 Efficiency Related Biases _. . . . . . . . . . . . . . . . . . . . 103

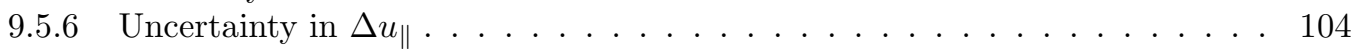

9.5.7 Uncertainties Due to Implementation of Unfolding . . . . . . . . . . . . . 105

9.5.8 Total Systematic Uncertainties Due to the Recoil System Simulation . . . . 105

10 Backgrounds $\quad 107$

10.1 Fake Rate . . . . . . . . . . . . . . . . . . . . . . 107

10.2 Backgrounds . . . . . . . . . . . . . . . . . . 107

$10.2 .1 \quad Z \rightarrow e e$ background . . . . . . . . . . . . . . . . 107

$10.2 .2 W \rightarrow \tau \nu$ Background . . . . . . . . . . . . . . . . . . 108

10.2 .3 QCD Background . . . . . . . . . . . . . . . . . . . . . . . . 108

10.2.4 Final Background Distributions . . . . . . . . . . . . . . . . 109

11 Full Monte Carlo Closure Tests 112 
12 Systematic Uncertainties 116

12.1 Theoretical Uncertainties _. . . . . . . . . . . . . . . . . . 116

12.1.1 PDF Uncertainties . . . . . . . . . . . . . . . . . . . . 116

12.1 .2 Boson $p_{\mathrm{T}}$ Model . . . . . . . . . . . . . . . . . . . 117

12.1.3 QED Model . . . . . . . . . . . . . . . . . . . . . . . . . 117

12.1 .4 W Mass . . . . . . . . . . . . . . . . . . . . . . . . . . . 118

12.2 Experimental Uncertainties . . . . . . . . . . . . . . . . . . . . . 118

12.2.1 Electron Energy Scale . . . . . . . . . . . . . . . . . . . . . . 118

12.2.2 Electron Energy Resolution . . . . . . . . . . . . . . . . . . . . 119

12.2 .3 Non-Linearity . . . . . . . . . . . . . . . . . . . . . . . . . . . . . . . 119

12.2 .4 Hadronic Model . . . . . . . . . . . . . . . . . . . . . . . . . . . . 119

12.2 .5 Efficiencies . . . . . . . . . . . . . . . . . . . . . . 119

12.2 .6 Backgrounds . . . . . . . . . . . . . . . . . . . . 120

12.3 Summary of Systematic Uncertainties . . . . . . . . . . . . . . . 120

13 Results 124

13.1 Final Result for the $W$ Boson Mass . . . . . . . . . . . . . . . . . . . . . . . . . . . 124

13.2 Cross-Checks and Comparison Plots for $M_{W} \ldots \ldots \ldots \ldots \ldots \ldots$. . . . . 124

13.3 Final Result for the $W$ Boson Decay Width . . . . . . . . . . . . . . . . . . . . . 134

13.4 Cross-Checks and Comparison Plots for $\Gamma_{W} \ldots \ldots \ldots \ldots \ldots \ldots \ldots$

14 Conclusions and Future Prospects 144

A Propagating the Effects of FSR in the Recoil File to $W \rightarrow e \nu$ Templates 149

A.1 Statistical Power and the Continuity Assumption . . . . . . . . . . . . . . . 151

$\begin{array}{ll}\text { B D } \varnothing \text { Author List } & 157\end{array}$

$\begin{array}{ll}\text { Bibliography } & 163\end{array}$ 


\section{LIST OF FIGURES}

2.1 The particles of the Standard Model. . . . . . . . . . . . . . . . . 4

2.2 Allowed interactions in the Standard Model. . . . . . . . . . . . . . . . . 5

2.3 Basic production and leptonic decay for $W / Z$ bosons with radiated gluons. . . . . 7

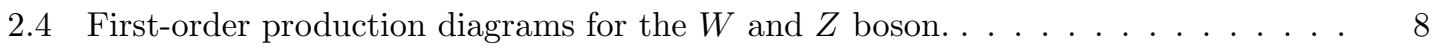

2.5 Momentum fractions carried by partons in a proton at $Q^{2}=10^{2} \mathrm{GeV} \ldots \ldots$. . . 9

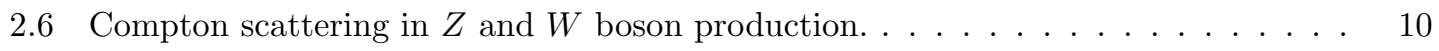

2.7 Initial-state gluon radiation in $Z$ and $W$ boson production. . . . . . . . . 11

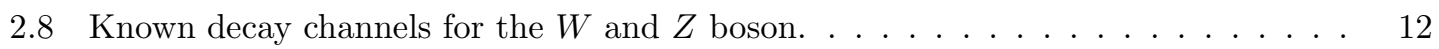

2.9 Higher order correction on the $W$ boson mass from a top-quark loop. . . . . . . . 13

2.10 Higher order correction on the $W$ boson mass from a Higgs loop. . . . . . . . . . 13

2.11 Constraints on the Higgs mass from $M_{W}$ and $M_{t o p} \ldots \ldots \ldots \ldots \ldots$

3.1 Anatomy of the transverse energy in the $D$ detector in a typical $W \rightarrow e \nu$ event. . $\quad 17$

$3.2 \quad M_{\mathrm{T}}$ (left) and $p_{\mathrm{T}}(e)$ (right) spectra for $W$ bosons with $p_{\mathrm{T}}^{W}=0$ (solid line), with the correct $p_{\mathrm{T}}^{W}$ spectrum (points), and with detector resolutions (shaded area). . . . . 18

3.3 Definition of $u_{\|}$and $u_{\perp}$. It should be noted that $u_{\|}$is defined as negative when opposite the electron direction. . . . . . . . . . . . . . . . . . . 19

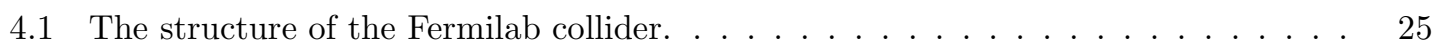

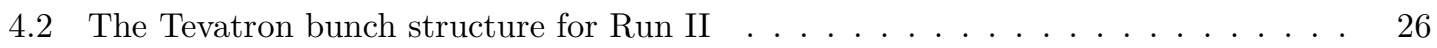

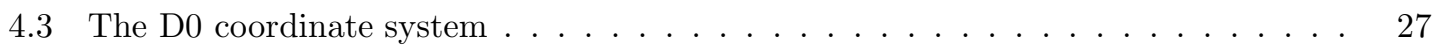

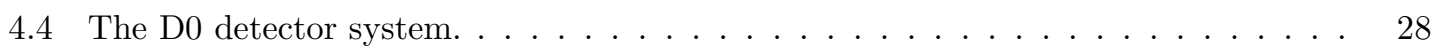

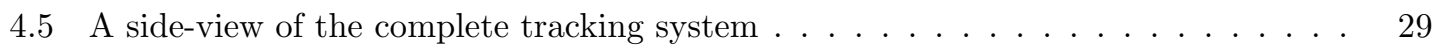

4.6 The D0 Silicon Microstrip Tracking detector. . . . . . . . . . . . . . . . . . . 30

4.7 A side-view of the D0 Central Fiber Tracking detector. . . . . . . . . . . . 31

4.8 A 3-dimensional view of the $\mathrm{D} 0$ calorimeters. . . . . . . . . . . . . . . 33

4.9 A side-view of one quarter of the detector, showing the projective tower structure in $\eta$ and depth. The lines extending from the center of detector correspond to constant

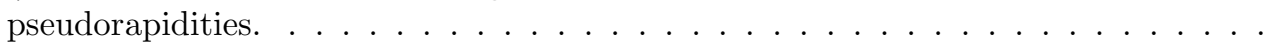

4.10 The schematic view of a typical unit cell, showing the gap structure, grounded absorber plates, and read-out boards. . . . . . . . . . . . . . 
4.11 Map showing the configuration of calorimeter cells as a function of detector $\eta$ and

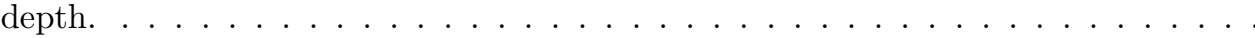

4.12 Schematic of the electronic read-out system. . . . . . . . . . . . . . . . . 37

4.13 Typical signal shapes and various stages of the electronic readout. . . . . . . . .

5.1 Comparison between the four EM fraction distributions of an uncalibrated tower (points) and the average distributions for the eta ring (solid) $\ldots \ldots \ldots \ldots$

5.2 Comparison between the four EM fraction distributions of an uncalibrated tower (points) and the average distributions for the eta ring (solid), after calibration . . .

5.3 Result of the calibration fit to data. The horizontal axis represents ieta and the vertical axis represents the fit result for $c_{\text {ieta }(j)}$. The grey areas show regions in which we do not attempt to determine $c_{\operatorname{ieta}(j)}$. The point at ieta $=-27$ represents the combined $c_{\text {ieta }(j)}$ for $-37 \leq$ ieta $\leq-27$, and the point at ieta $=27$ represents the combined $c_{\operatorname{ieta}(j)}$ for $27 \leq$ ieta $\leq 37$ (see text). The triangles represent the result for data taken before the Sept-Nov 2003 shutdown, and the dots represent the result for data taken after that shutdown. . . . . . . . . . . . . . . . . .

5.4 Temperature plots of the inter-calibration constants for EM1 (upper left), EM2 (upper right), EM3 (lower left), and EM4 (lower right). . . . . . . . . . . .

5.5 The Width of the Z-peak for two CC elections, before (top) and after (bottom) the in-situ calibration was performed. Resolution improves by roughly $10 \%$. . . . . .

5.6 The average energy deposited at a function of depth (in radiation lengths) for 45 $\mathrm{GeV}$ single-electrons at normal incidence, as estimated using a parameterized shower

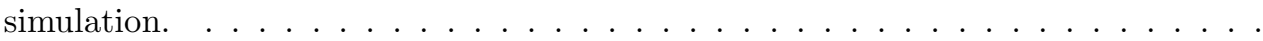

5.7 The energy deposited at a function of depth (in radiation lengths) for $45 \mathrm{GeV}$ single-electrons at normal incidence (left) and $\eta=1.0$ (right) as estimated using a parameterized shower simulation. Each line represents one particular shower drawn from a parameterized model for shower fluctuations [63]. One clearly sees the shift of the shower earlier in the calorimeter as the angle of incidence increases. . . . . .

5.8 The energy deposited at a function of depth (in radiation lengths) for $45 \mathrm{GeV}$ (left) and $5 \mathrm{GeV}$ (right) single-electrons at normal incidence. Each line represents one particular shower drawn from a parameterized model for shower fluctuations [63]. One clearly sees the large shift of the shower earlier in the calorimeter at lower energies. . . . . . . . . . . . . . . . . .

5.9 Fractional Energy Resolution for $45 \mathrm{GeV}$ electrons as a function of physics $\eta$, in GEANT Monte Carlo. The data points are generated from full GEANT Monte Carlo of single electrons. The solid curve is a fit using the parameterization described in Chapter 7 and the dashed line shows a $1 / \sqrt{\sin \theta}$ dependence. . . . . . . . . .

5.10 The fractional energy resolution due to sampling effects for electrons at normal incidence as a function of energy. The data points are generated from full GEANT Monte Carlo of single electrons. The dashed line represents a $1 / \sqrt{E}$ dependence and the solid line is a fit using the new parameterization described in Chapter 7 . .

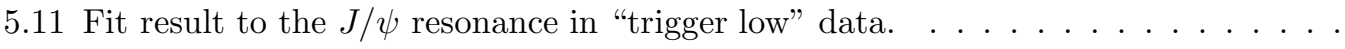


5.12 Sampling resolution measured at the $J / \psi$ versus kinematic category. The dark error bars indicate the systematic uncertainty and the light error bars are the total uncertainty. The dashed line indicates where the GEANT derived sampling resolution is. The discrepancy points to missing material in the full simulation. . . . . . . . . .

5.13 Likelihood function for the final fit results for missing $X_{0}$ in the full detector simulation. 56

5.14 Results of fits for missing $X_{0}$ in each EM layer, independently. $\ldots \ldots \ldots \ldots$

5.15 Comparison between data and an MC model of the longitudinal shower profile for category $23 Z$ data before missing material is added. . . . . . . . . . .

5.16 Comparison between data and an MC model of the longitudinal shower profile for category $23 Z$ data after missing material is introduced. . . . . . . . . . . .

7.1 The distribution of $M_{\mathrm{Z}}$ vs. $f_{\mathrm{Z}}$ in full Monte Carlo events. . . . . . . . . . 67

7.2 The profile plot of $M_{\mathrm{Z}}$ vs. $f_{\mathrm{Z}}$ in full Monte Carlo events. . . . . . . . . 67

7.3 Log plot of the energies in the window of towers rotated to a position azimuthally adjacent to the electron window. . . . . . . . . . . . . .

7.4 Figures showing the dependence on instantaneous luminosity (top) and $u_{\|}$of the underlying energy correction (bottom) . . . . . . . . . . . .

7.5 Scatter plot of the shift between track position and calorimeter cluster center as a function of extrapolated track $\phi$-mod. . . . . . . . . . . . . . . . .

7.6 Profile plot of the shift between track position and calorimeter cluster center as a function of extrapolated track $\phi$-mod. . . . . . . . . . . . . . .

7.7 The selection efficiencies for electrons in FSR events as a function of the fraction of electron energy carried by the leading photon, for bins in $\Delta R(e \gamma)$, proximity of the

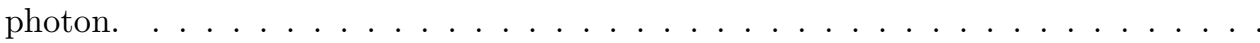

7.8 The fraction of FSR photon energy lost from the electron as a function of Efrac $\gamma$

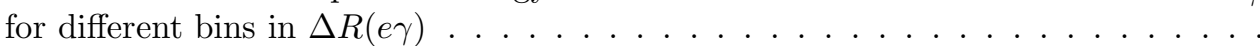

8.1 The trigger efficiency versus electron $p_{\mathrm{T}}$ for four different run trigger periods: v11 (upper left), v12 (upper right), v13 (lower left, and v14 (lower right). . . . . . . .

8.2 The tracking efficiency as a function of $\eta$ and vertex $z$ position shown as a lego plot

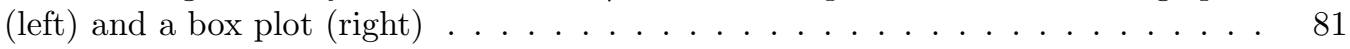

8.3 The Hmatrix efficiency versus $\eta_{\text {det }} \ldots \ldots \ldots \ldots \ldots \ldots \ldots$. . . . . . . . . 82

8.4 The distribution of $M_{\mathrm{Z}}$ vs. $f_{\mathrm{Z}}$ in full Monte Carlo events. . . . . . . . . . . 83

8.5 The $u_{\|}$efficiency for $Z \rightarrow e e$ (top) and $W \rightarrow e \nu$ (bottom) events for full (black points) and fast (blue points) MC. Good agreement is found. . . . . . . . . . . 85

8.6 The overall reconstruction efficiency as a function of SET for $W$ and $Z$ events. . . $\quad 86$

8.7 The $p_{\mathrm{T}}(e)$ based corrections on the overall SET efficiency, as a function of SET. . . 87 
8.8 Left: $p_{T}$ dependence of HMx efficiency for CC electrons in data(black) and full $\mathrm{MC}(\mathrm{red})$; Right: Ratio between the black and red curve in the left plot. . . . . . .

8.9 Left: $p_{T}$ dependence of track match efficiency for CC electrons in data(black) and full MC(red); Right: Ratio between the black and red curve in the left plot. . . . .

9.1 The distribution of the probabilities that a reconstructed $p_{T}^{Z}$ of $7 \mathrm{GeV}$ with corresponding $u_{T}$ of $3.5 \mathrm{GeV}$ came from various true $p_{T}^{Z}$ bins. . . . . . . . .

9.2 Mean recoil $u_{T}$ versus true $p_{T}^{Z}$ (black filled points) compared with mean recoil $u_{T}$ versus the estimate of the true $p_{T}^{Z}$ (red open boxes) when using (a) the two smeared electrons directly and (b) using the unfolded map. . . . . . . . . . . . . . . .

9.3 Mean projection of the recoil along the boson direction versus true $p_{T}^{Z}$ (black filled points) compared with mean projection of the recoil along the boson direction versus the estimate of the true $p_{T}^{Z}$ (red open boxes) when using (a) the two smeared electrons directly and (b) using the unfolded map. . . . . . . . . . . . .

9.4 RMS of the opening angles between the recoil and the boson versus true $p_{T}^{Z}$ (black filled points) compared with RMS of the opening angle between the recoil and the boson versus the estimate of the true $p_{T}^{Z}$ (red open boxes) when using (a) the two smeared electrons directly and (b) using the unfolded map. . . . . . . . . . . .

9.5 RMS of the recoil $u_{T}$ versus true $p_{T}^{Z}$ (black filled points) compared with RMS of the recoil $u_{T}$ versus the estimate of the true $p_{T}^{Z}$ (red open boxes) when using (a) the two smeared electrons directly and (b) using the unfolded map. . . . . . . . . . .

9.6 Angle between the measured recoil and the true $Z$ boson direction (solid line) and angle between the measured recoil and the measured $Z$ boson direction (points with error bars) when using (a) the two smeared electrons directly and (b) using the unfolded map for $Z$ events with a true $p_{T}^{Z}$ of 4.0 to $4.25 \mathrm{GeV}$. . . . . . . . . .

9.7 Mean $W$ boson mass measured in 100 ensemble tests for each template generated from a recoil file. The black line is a fit using a Gaussian function. All toy models were generated with an input $W$ boson mass of $80.419 \mathrm{GeV}$ and the fitted gaussian function has a mean value of $80.420 \pm 0.001 \mathrm{GeV}$ and the fitted gaussian width is

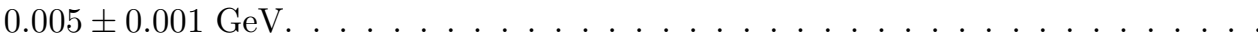

9.8 Mean $W$ boson width measured in 100 ensemble tests for each template generated from a recoil file. The black line is a fit using a Gaussian function. All toy models were generated with an input $W$ boson width of $2.039 \mathrm{GeV}$ and the fitted gaussian function has a mean value of $2.040 \pm 0.001 \mathrm{GeV}$ and the fitted gaussian width is $0.040 \pm 0.003 \mathrm{GeV} \ldots \ldots \ldots \ldots \ldots \ldots \ldots$

10.1 The three background shapes for the $M_{\mathrm{T}}$ distribution: QCD (black), $Z \rightarrow e e$ (red),

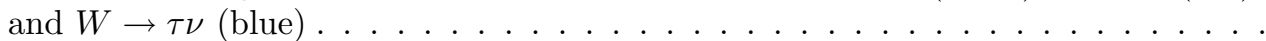

10.2 The three background shapes for the $E_{T}$ distribution: QCD (black), $Z \rightarrow e e$ (red),

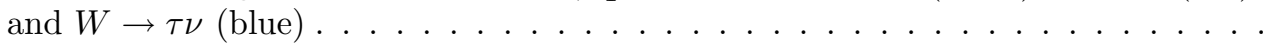

10.3 The three background shapes for the $p_{\mathrm{T}}(e)$ distribution: QCD (black), $Z \rightarrow e e$ (red), and $W \rightarrow \tau \nu$ (blue) $\ldots \ldots \ldots \ldots \ldots \ldots$ 
11.1 Comparison plots between full MC (points) and fast $\mathrm{MC}$ (lines) for the $\mathrm{W} M_{\mathrm{T}}$ distribution (top), $\mathrm{W}$ electron $p_{\mathrm{T}}$ distribution (middle), and MET distribution (bottom). Corresponding $\chi$ plots are shown on the right side. . . . . . . . .

11.2 Comparison plots between full MC (points) and fast MC (lines). Top left: Z mass distribution. Top right: $\mathrm{Z} p_{\mathrm{T}}$ distribution, reconstructed from the di-electron $p_{\mathrm{T}}$. Lower left: Z Recoil $p_{\mathrm{T}}$ distribution. Lower right: Z electron $p_{\mathrm{T}}$ distribution. . . .

12.1 Ratio of the $p_{\mathrm{T}}$ dependence of the HMx efficiency between data and full MC (points), fit with the nominal fit function (black line) and with plus (red) and minus (blue) 1 sigma on the $p_{\mathrm{T}}$ dependent slope. . . . . . . . . . . . . .

13.1 Comparison of $Z \rightarrow e e$ invariant mass spectrum between data and fast MC. . . . 126

13.2 Comparison of $Z \rightarrow e e p_{\mathrm{T}}(e)$ spectrum between data and fast MC. . . . . . .

13.3 Comparison of $Z \rightarrow e e$ recoil $p_{\mathrm{T}}$ spectrum between data and fast MC. . . . . . .

13.4 Comparison plots between data (points) and fast $\mathrm{MC}$ (lines) for the $\mathrm{W} M_{\mathrm{T}}$ distribution. . . . . . . . . . . . . . . . . . . . .

13.5 Negative log-likelihood plot for the $W$ boson mass fit to the $M_{\mathrm{T}}$ observable. . . .

13.6 Comparison plots between data (points) and fast $\mathrm{MC}$ (lines) for the $p_{\mathrm{T}}(e)$ distribution. 130

13.7 Comparison plots between data (points) and fast MC (lines) for the $E_{T}$ distribution. 131

13.8 Left: $\mathrm{W}$ mass as measured from $M_{\mathrm{T}}, p_{\mathrm{T}}(e)$, and $E_{T}$ observables for two independent run periods. Middle: The equivalent fits for the $\mathrm{Z}$ mass from the di-electron invariant mass spectrum. Right: The fractional change in the $\mathrm{W} / \mathrm{Z}$ mass ratio measured from $M_{\mathrm{T}}, p_{\mathrm{T}}(e)$, and $E_{T}$ observables. The green line represents the nominal value. . . .

13.9 Left: $\mathrm{W}$ mass as measured from $M_{\mathrm{T}}, p_{\mathrm{T}}(e)$, and $E_{T}$ observables for two data subsets, corresponding to different luminosities. Middle: The equivalent fits for the $\mathrm{Z}$ mass from the di-electron invariant mass spectrum. Right: The fractional change in the $\mathrm{W} / \mathrm{Z}$ mass ratio measured from $M_{\mathrm{T}}, p_{\mathrm{T}}(e)$, and $E_{T}$ observables. The green line represents the nominal value. . . . . . . . . . . . . . . . .

13.10Left: $\mathrm{W}$ mass as measured from $M_{\mathrm{T}}, p_{\mathrm{T}}(e)$, and $E_{T}$ observables for two data subsets, corresponding to positive and negative $u_{\|}$. Middle: The equivalent fits for the $\mathrm{Z}$ mass from the di-electron invariant mass spectrum. Right: The fractional change in the $\mathrm{W} / \mathrm{Z}$ mass ratio measured from $M_{\mathrm{T}}, p_{\mathrm{T}}(e)$, and $E_{T}$ observables. The green line represents the nominal value.

13.11Left: $\mathrm{W}$ mass as measured from $M_{\mathrm{T}}, p_{\mathrm{T}}(e)$, and $E_{T}$ observables for two different $U_{T}$ cuts. Middle: The equivalent fits for the $\mathrm{Z}$ mass from the di-electron invariant mass spectrum. Right: The fractional change in the $\mathrm{W} / \mathrm{Z}$ mass ratio measured from $M_{\mathrm{T}}, p_{\mathrm{T}}(e)$, and $E_{T}$ observables. The green line represents the nominal value. . . .

13.12Left: $\mathrm{W}$ mass as measured from $M_{\mathrm{T}}, p_{\mathrm{T}}(e)$, and $E_{T}$ observables for two different $\eta_{\text {det }}$ cuts. Middle: The equivalent fits for the $\mathrm{Z}$ mass from the di-electron invariant mass spectrum. Right: The fractional change in the $\mathrm{W} / \mathrm{Z}$ mass ratio measured from $M_{\mathrm{T}}, p_{\mathrm{T}}(e)$, and $E_{T}$ observables. The green line represents the nominal value. . . . 
13.13Comparison plots between data (points) and fast $\mathrm{MC}$ (lines) for the $\mathrm{W} M_{\mathrm{T}}$ distribution on a $\log$ scale. . . . . . . . . . . . . . . . . . . . .

13.14Comparison plots between data (points) and fast MC (lines) in the tail of the W $M_{\mathrm{T}}$ distribution on a $\log$ scale. . . . . . . . . . . . . . . . .

13.15Comparison plots between data (points) and fast MC (lines) in the tail of the W $p_{\mathrm{T}}(e)$ distribution on a $\log$ scale. . . . . . . . . . . . . . . . . . .

13.16Comparison plots between data (points) and fast MC (lines) in the tail of the W $E_{T}$ distribution on a $\log$ scale. . . . . . . . . . . . . . . .

13.17Plots showing the blinded $W$ boson width measurements for various sub-sets of the data: separated into independent run-ranges (upper left), for different $U_{T}$ cuts (upper right), separated into independent sets of instantaneous luminosity (middle left), for different $\eta_{\text {det }}$ cuts (middle right), separated into positive and negative $u_{\|}$ (lower left), and for different $\phi_{m o d}$ cuts (lower right). . . . . . . . . . . . .

13.18Plots showing the variations of the measured $W$ boson width for different values for the lower range of the fit (top) and different values of the upper range (bottom) with statistical error-bars. The yellow bands correspond to the fits using the Recoil Library Method, and the red points correspond to the Parameterized Recoil Model.

13.19Fraction of events in the tail region $[100,200]$ to events in the body $[0,100]$ of the $W$ boson $M_{\mathrm{T}}$ distribution versus blinded $W$ boson width for fast MC templates. The black lines represent the known tail-to-body ratio for the data, and $\pm 1 \sigma$ of that ratio. The data line intersects with the graph at roughly $2.27 \mathrm{GeV}$, which agrees with the blinded width value obtained through negative log-likelihood fits. . . . . .

13.20Plots showing the variations of the measured $W$ boson width for different values for the lower range of the fit (top) and different values of the upper range (bottom) with statistical error-bars. The yellow bands correspond to the fits using the Recoil Library Method, and the red points correspond to the Parameterized Recoil Model.

14.1 The new D0 $W$ boson mass measurement compared with various other measurements and the updated world average (yellow band). . . . . . . . . . . . .

14.2 The new D0 $W$ boson width measurement compared with various other measurements and the current world average (yellow band). . . . . . . . . . . . . .

14.3 Plots showing the restricted regions where the Higgs mass is expected. . . . . . . . 146

A.1 The projection of unmerged photon $p_{T}$ along the direction of the $Z$ boson, versus the $p_{T}$ of the $Z \ldots \ldots \ldots \ldots \ldots \ldots \ldots \ldots$

A.2 Distribution of the means of $\eta$-imbalance distributions for 1000 toy $W$ sets, each generated from an independent recoil histogram containing $18 \mathrm{k}$ entries, with no hadronic smearing using the parameterized approach (a) and using the binned approach (b). Note how the RMSs of these two distributions are roughly equal. Since fluctuations in the mean $\eta$-imbalance represent fluctuations in the mean response for a given recoil file, the parameterized and binned approaches both model the mean response with the same precision. . . . . . . . . . . . . . . . . 
A.3 The RMS's of the of $\eta$-imbalance distributions for 1000 toy $W$ sets, each generated from and independent recoil histogram containing 18k events, and no hadronic resolution. The solid line corresponds to the RMSs of the $\eta$-imbalance distributions using the parameterized approach. The dashed line shows the RMSs using the binned approach. Note that the average RMS of the $\eta$-imbalance distribution for the binned approach is ten times larger than that of the parameterized approach. . 
List of Abbreviations

$\begin{array}{ll}\text { SM } & \text { Standard Model } \\ \text { EM } & \text { Electromagnetic } \\ \text { EW } & \text { Electroweak } \\ \text { MET } & \text { Missing Transverse Energy } \\ \text { SMT } & \text { Silicon Microstrip Tracker } \\ \text { CFT } & \text { Central Fiber Tracker } \\ \text { VLPC } & \text { Visible Light Photon Counter } \\ \text { WLS } & \text { Wave length Shifting } \\ \text { CPS } & \text { Central Preshower } \\ \text { FPS } & \text { Forward Preshower } \\ \text { MIP } & \text { Minimum Ionizing Particle } \\ \text { EC } & \text { Endcap Calorimeter } \\ \text { CC } & \text { Central Calorimeter } \\ \text { FH } & \text { Fine Hadronic } \\ \text { CH } & \text { Coarse Hadronic } \\ \text { ICD } & \text { Inner Cryostat Detector } \\ \text { ICR } & \text { Inner Cryostat Region } \\ \text { PDT } & \text { Proportional Drift Tube } \\ \text { MDT } & \text { Mini Drift Tube } \\ \text { PMT } & \text { Photo Multiplier Tube } \\ \text { MUC } & \text { Central Muon Detector } \\ \text { MUF } & \text { Forward Muon Detector } \\ \text { L1, L2, L3 } & \text { Level 1, 2, 3 (trigger) } \\ \text { ADC } & \text { Analog to Digital Converter } \\ \text { DCA } & \text { Distance of Closest Approach } \\ \text { HTF } & \text { Histogram Track Finder } \\ \text { AA } & \text { Alternative Algorithm } \\ \text { SET } & \text { Scalar E } \\ & \\ & \\ & \end{array}$




\section{Chapter 1}

\section{Introduction}

The $W$ and $Z$ bosons are the massive gauge bosons that, along with the photon, mediate electroweak interactions. The properties of these bosons are not only interesting in their own right; they are also important because they could point to unobserved physics such as the spontaneous symmetry breaking through which the $W$ and $Z$ bosons are hypothesized to acquire mass. The mass of the $W$ boson is sensitive to radiative corrections from the Higgs and potentially other exotic particles. Precision measurements of the $W$ boson mass, combined with those of the top quark mass and other electroweak observables, can place limits on the expected mass of the Standard Model (SM) Higgs boson.

This dissertation presents a measurement of the $W$ boson mass $\left(M_{W}\right)$ and decay width $\left(\Gamma_{W}\right)$ using $W \rightarrow e \nu$ events from $1 \mathrm{fb}^{-1}$ of collider data collected in the $\mathrm{D} \varnothing$ detector at the Tevatron. Extracting $M_{W}$ and $\Gamma_{W}$ from $W \rightarrow e \nu$ requires a physics model for the production and decay of the $W$ boson, as well for detector effects in measuring the relevant observables. The two main components of a $W \rightarrow e \nu$ decay are the decay electron and the measured hadronic recoil, which consists of the "hard recoil" that balances the momentum of the boson transverse to collision and any other hadronic energy produced in the detector. The electron is a single particle, with a well-understood energy response in a collider detector like DØ. The measured hadronic recoil, on the other hand, is a complicated admixture of several different effects, making it difficult to model on first principles. We have developed an alternative, heuristic approach to modelling the recoil. Simulated $W \rightarrow e \nu$ events are overlaid with data recoils chosen from a library of $Z \rightarrow e e$ events alligned to match the direction and transverse momentum of the generated $W$ boson. This approach requires no a priori understanding of the recoil, has no tunable parameters, and reproduces all of the complex detector effects observed in data. This method provides an excellent cross-check on the $W$ boson mass measurement, and is a useful alternative for the measurement 
of $\Gamma_{W}$, where the recoil model is the dominant systematic.

Versions of the Recoil Library Method have been proposed in the past [1]. What is of particular interest in this dissertation is a Bayesian unfolding technique for removing detector effects on the measured momentum of the $Z$ boson. Implementation of the recoil library method requires that the data recoil library describe the measured recoil in relation to the true boson momentum and direction. However, data gives the measured recoil in relation to the measured $Z$ boson $p_{\mathrm{T}}$, as reconstructed from the two decay electrons, which can fluctuate non-trivially from the true momentum in both magnitude and direction. Early use of recoil libraries neglected this effect, since statistical uncertainties dominated. To the level of precision demanded at the Run II Tevatron and expected at the LHC, this effect is large compared to statistical uncertainties.

In this dissertation we will cover the entire Run IIa program for measuring the $W$ boson mass and width, with special attention paid to the novel recoil model used.

In the next chapter, we will briefly survey the important topics in theoretical particle physics. Chapter 3 provides a detailed discussion of the $M_{W}$ and $\Gamma_{W}$ measurement strategy. Chapter 4 contains a description of the Tevatron accelerator and the DØ detector. A great deal of work was done in Run II to improve our understanding, and thus the perfomance, of the upgraded detector. We describe this work in Chapter 5. The data are described in Chapter 6. Chapter 7 outlines the parametric model used to simulate electron measurement, and Chapter 8 covers the efficiency models. Chapter 9 outlines the implementation of the heuristic Recoil Library Method and its systematics, as well as a brief treatment of the parametric recoil model. Chapter 10 discusses the relevant backgrounds. We test our methodology on realistic a GEANT-based $W \rightarrow e \nu$ simulation in Chapter 11. Chapter 12 discusses systematic uncertainties for the $M_{W}$ and $\Gamma_{W}$ measurements, and Chapter 13 presents the results. Finally in Chapter 14 we discuss conclusions and future prospects from the measurements. The appendices contain several detailed discussions relevant to the recoil model presented in this dissertation. 


\section{Chapter 2}

\section{Review of Theoretical Particle Physics}

In this Chapter, we will review aspects of theoretical physics relevant to the measurement of the $W$ boson mass and width, and provide context for its significance in the larger program of experimental particle physics.

\subsection{The Standard Model}

The goal of elementary particle physics is to provide a consistent, first-principles description of the fundamental constituents of matter and energy with minimal starting assumptions and input parameters. The "Standard Model" is the name given to our best complete description to date. Further reading on the topic can be found in $[2,3,4]$.

The Standard Model divides fundamental matter particles into quarks and leptons, both spin- $1 / 2$ fermions. The quarks and leptons are then further divided into three generations, each consisting of two quarks, one charged lepton, and one nearly massless neutral lepton, known as a neutrino. The quarks and charged leptons in each generation are successively more massive. All known, stable matter is comprised of the first and lightest generation, which consists of the up-quark, down-quark, electron, and electron neutrino.

The fundamental interactions between matter particles are traditionally divided into four basic forces: strong force, electromagnetism, weak force, and gravitation, listed in order of decreasing strength. The quantum mechanical model of these forces describes them as the exchange of mediating particles called gauge bosons. The properties of these gauge bosons determine the nature of the fundamental forces. Electromagnetism is mediated by a massless spin-1 particle known as the photon (represented by $\gamma$ ), which couples to electric charge. The quantum theory describing electromagnetic interactions, Quantum Electrodynamics or QED, is a triumph of modern physics, able to predict electromagnetic phenomena with remarkable precision. The weak-force 
Elementary

Particles

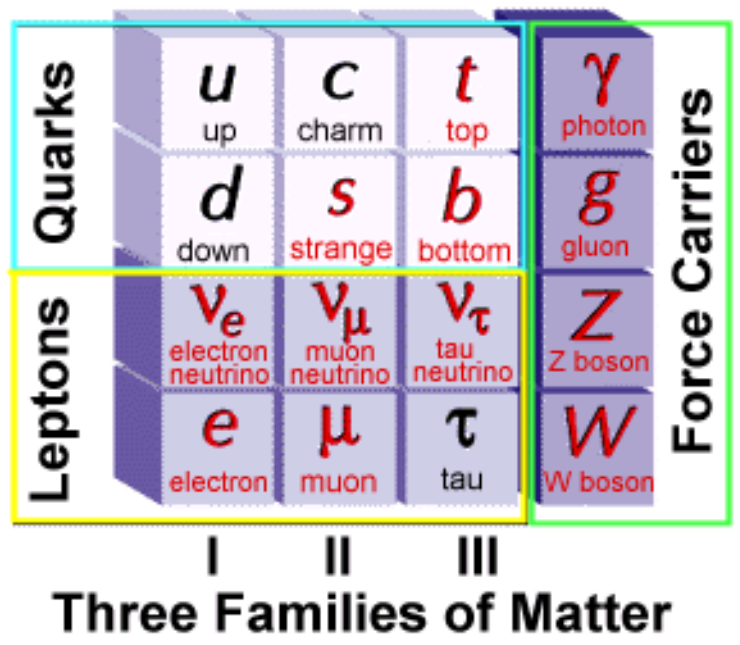

Figure 2.1: The particles of the Standard Model.

is a short-range force carried by the massive $(\approx 80 \mathrm{GeV})$ charged $W^{ \pm}$boson and the massive $(\approx 90$ $\mathrm{GeV}$ ) neutral $Z^{0}$ boson. The weak force couples to weak isospin. "Electroweak Theory" provides a unified framework for describing both the electromagnetic and weak forces, and is a major pillar of the Standard Model. The strong force, carried by massless bosons called gluons, couples to 3 "color" charges, so named as a matter of convention and not because of any connection with optical color. Like the weak force, the strong force has a very short range. The theoretical framework for understanding strong force is called Quantum Chromodynamics or QCD. The gravitational force, which couples to mass, is not described by the Standard Model.

Quarks are strongly interacting particles, although they can also interact electroweakly. The strong force only permits "color neutral" bound states of either quark-antiquark pairs or triplets of three quarks (each one a different color), and has the unusual property that its strength increases with distance. As a consequence, single quarks cannot stably exist. Quark matter is called "hadronic" matter and bound states are called hadrons. The most common hadrons are protons and neutrons which consist of up and down quarks (uud and ddu, respectively).

Leptons interact primarily through electroweak interactions, with charged leptons interact- 
ing both electromagnetically and weakly. Charge-neutral neutrinos can only interact via the weak force, which is why they are so difficult to detect. The gauge bosons can also interact with each other, while gluons and $W$ bosons can even self-couple. Figure 2.2 shows the possible interactions allowed between quarks, leptons, and bosons.

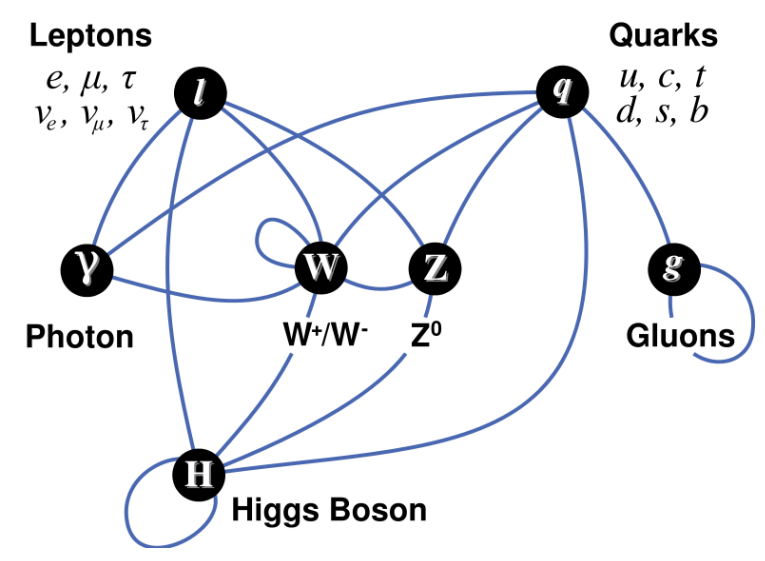

Figure 2.2: Allowed interactions in the Standard Model.

One of the outstanding questions of electroweak physics is the origin of mass. Gauge theoretic descriptions of the electroweak force, built around symmetry arguments, predict three massless gauge bosons. However, in nature we observe one massless photon and two very massive weak bosons. The Standard Model framework for addressing the origin of this mass hypothesizes the existence of a quantum field that spontaneously breaks electroweak symmetry, introducing mass terms into the Lagrangian [5]. This Higgs field is given by the Lagrangian:

$$
\mathcal{L}_{\text {Higgs }}=\left(D^{\mu} \phi\right)^{\dagger}\left(D^{\mu} \phi\right)-V\left(\phi^{\dagger} \phi\right)
$$

where $\mathrm{V}$ has the form,

$$
V\left(\phi^{\dagger} \phi\right)=-\mu^{2}\left(\phi^{\dagger} \phi\right)+\frac{1}{4}|\lambda|\left(\phi^{\dagger} \phi\right)^{2}
$$

This potential has a non-zero vacuum expectation value, and the degenerate set of true minima do not respect the electroweak symmetry. In quantum field theory all physical fields must be expanded around the real vacuum. Gauge freedom allows us to chose one of the degenerate 
minima about which to expand the new electro-weak fields. Quadratic terms in the new Lagrangian built around this minimum introduce masses to two of the three electroweak bosons and correctly predict their relative masses (for further reading, see [6, 7]):

$$
\mathrm{M}_{Z}=M_{W} / \cos \theta_{W}, \quad \mathrm{M}_{A}=0
$$

where $\theta_{W}$ is a parameter called the "weak mixing angle". The corresponding boson to the Higgs field is the last remaining particle predicted by the Standard Model that has not yet been observed. Ongoing searches at LEP and the Tevatron have greatly reduced the range of masses a standard model (SM) Higgs is expected to have. A low mass Higgs could be detected at the Tevatron, and will almost certainly be discovered at the LHC, if it exists.

Quantum Electroweak Theory predicts that, at the $\approx 2 \%$ level, the $W$ boson mass is sensitive to higher order corrections from Higgs loops. Precision measurements of $M_{W}$, combined with those of the top quark mass significanly constrain the likely mass of the SM Higgs, if it exists. These results are of great importance to the physics world. They stand among the major goals of Run II at the Tevatron and could be among its lasting legacies. In addition the $W$ boson mass would be subject to corrections from other exotic physics beyond the Standard Model, such as Supersymmetry.

The $W$ and $Z$ boson were first hypothesized by Glashow, Salaam, and Weinberg in the 1960's [8]. They were first observed by the UA1 and UA2 collaborations in the 1980's [9]. With roughly 17 million $\mathrm{Z}$ decays collected at LEP, and 600,000 at SLAC, the properties of the $\mathrm{Z}$ are very well measured. For example, the $Z$ mass is known to a precision of $2.1 \mathrm{MeV}$ [10]. In contrast, because of the low cross-section for $e-e+\rightarrow W^{+} W^{-}$at the lepton collider, measurements of the $W$ boson mass remain less precise, with an uncertainty on the current world average of only 25 $\mathrm{MeV}[11]$.

The measurements presented in this dissertation represent a continuation of these electroweak efforts and a modest step towards the completion of electroweak theory. 


\subsection{Production and Decay of the $\mathrm{W}$ and $\mathrm{Z}$ Boson}

In hadron colliders, at leading order in $\alpha_{s}$, the $Z$ and $W$ bosons are produced by quark anti-quark annihilation with no momentum in the plane transverse to the beam. However, higher order processes can include radiated gluons or quarks that balance the transverse momentum of the boson. These processes are similar for both the $W$ and $Z$ bosons, as are the resulting boson $p_{\mathrm{T}}$ spectra, with maxima around $3 \mathrm{GeV}$ and broad tails in the high $p_{\mathrm{T}}$ region [13]. Figure 2.3 shows an example diagram for the production of $W / Z$ bosons with two radiated gluons. Figure 2.4 shows the typical first-order process.

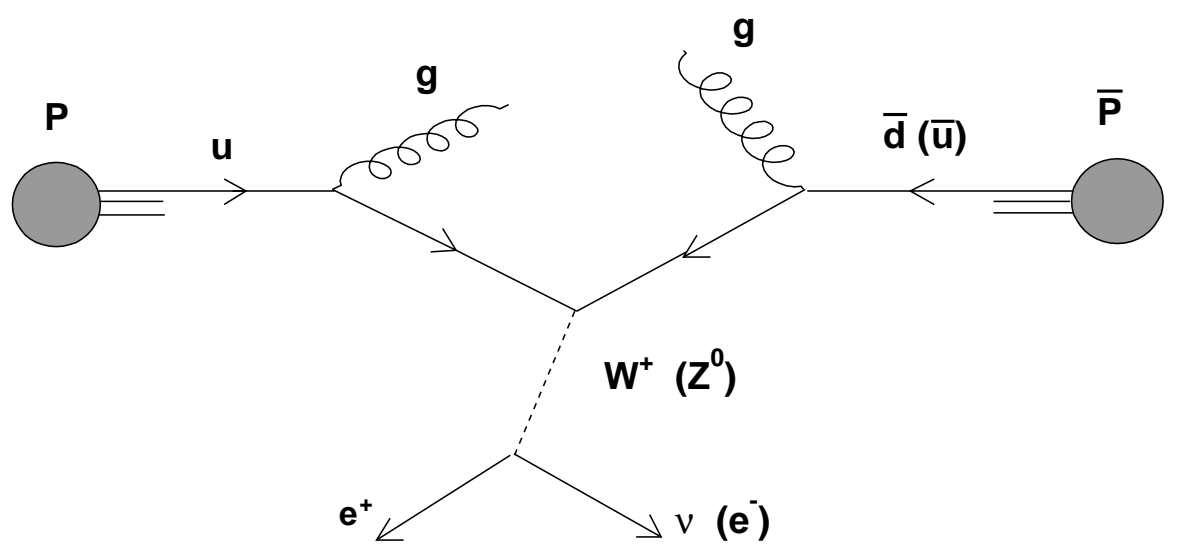

Figure 2.3: Basic production and leptonic decay for $W / Z$ bosons with radiated gluons.

The quark anti-quark pairs that produce the $W$ and $Z$ bosons can come from a $p \bar{p}$ collisions. Conceptually the proton and anti-proton are imagined as consisting of three quarks each, uud in the case of the proton and $\bar{u} \bar{u} \bar{d}$ for the anti-proton. In actuality, these hadrons contain not only the three "valence" quarks, but also gluons, which carry roughly half of the proton momentum, and a flux of virtual quark and anti-quark pairs, called "sea" quarks. Both gluons and sea quarks can contribute to $W$ and $Z$ boson production. In addition, once the proton and anti-proton are 

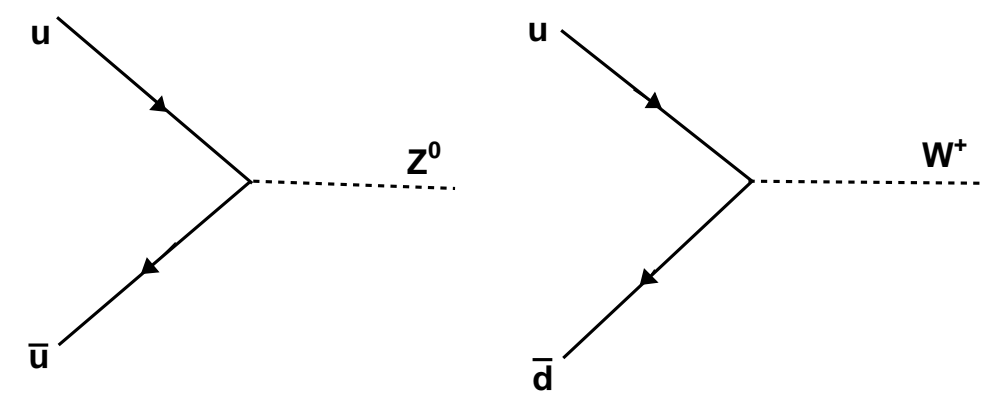

Figure 2.4: First-order production diagrams for the $W$ and $Z$ boson.

fragmented after producing a $W$ or $Z$ boson, the sea quarks and gluons help to drive "hadronization" of the remaining quarks into color-neutral pairs and triplets. The hadronic material from the partons not involved in boson production typically consists of 6 isolated, low $p_{\mathrm{T}}$ pions per unit rapidity at Tevatron energies, and constitutes what is known as the "underlying event" [14].

The parton-level cross-section $\left(\sigma_{i j}(\hat{s})\right)$ for two spin $1 / 2$ quarks producing a spin-1 boson are given by the Relativistic Bret-Wigner Resonance:

$$
\sigma_{i j}(\hat{s})=\frac{1}{3} \frac{\left|V_{i j}\right|^{2}}{3 \pi}\left(\frac{G M_{V}^{2}}{\sqrt{2}}\right)^{2} \frac{\hat{s} \Gamma_{0}^{2} / M_{V}^{2}}{\left(\hat{s}-M_{V}^{2}\right)^{2}+\left(\hat{s} \Gamma_{0} / M_{V}\right)^{2}}
$$

where $\hat{s}$ is the parton center-of-mass energy, $G$ is the Fermi constant $V_{i j}$ is the CKM Matrix named after Cabbibo, Kobayashi, and Maskawa that describes quark mixing, and $M_{V}$ and $\Gamma_{0}$ are the mass and width of boson, respectively.

While the Tevatron collides the protons and anti-protons at $\sqrt{(s)}=1.96 \mathrm{TeV}$, with center of mass in the lab frame, the constituent quarks or gluons in each parton carry varying fractions of the total parton momentum. Consequently, the quark or gluon collisions that produce the bosons are not typically at rest with respect to the detector, nor is the collision energy typically 
at the full $1.96 \mathrm{TeV}$. The momentum fractions $x_{1}$ and $x_{2}$ of two partons $q_{1}=x_{1} P_{1} q_{2}=x_{2} P_{2}$ where $P_{1}$ and $P_{2}$ are the proton and anti-proton momenta. At the center-of-mass frame of the two partons, $\sqrt{\hat{s}}=\sqrt{x_{1} x_{2} s}$. Figure 2.5 shows the momentum fractions carried by partons in a proton at $Q^{2}=10^{2} \mathrm{GeV}$

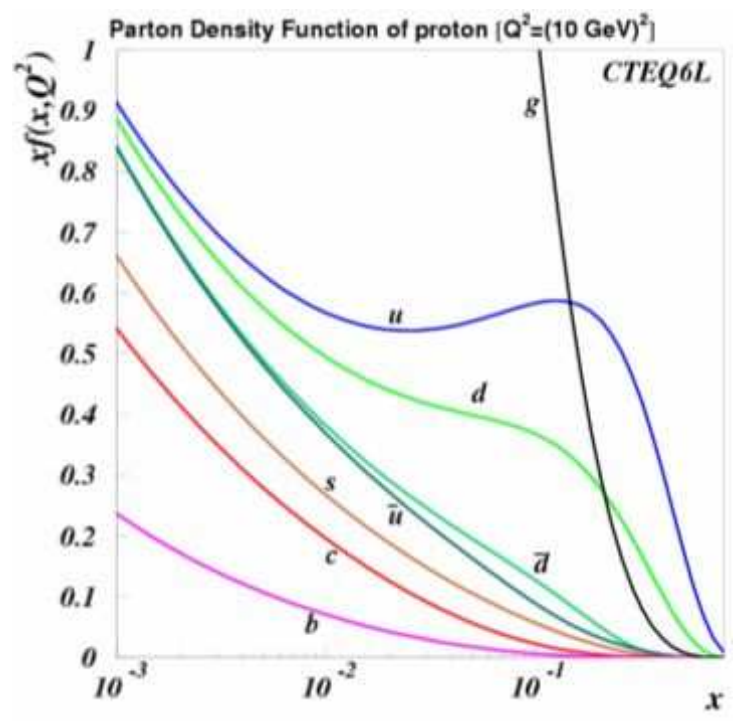

Figure 2.5: Momentum fractions carried by partons in a proton at $Q^{2}=10^{2} \mathrm{GeV}$.

Then, the total cross-section is then given by the sum over all parton-level cross-sections, convoluted with the parton distribution functions, giving the momenta fractions of all possible parton combinations:

$$
\sigma=\sum_{i j} \int d x_{1} d x_{2} f_{i}\left(x_{1}, Q^{2}\right) f_{j}\left(x_{2}, Q^{2}\right) \hat{\sigma}(i j)
$$

and the differential cross-section with respect to boson $p_{\mathrm{T}}$ and rapidity $(y)$ is given by:

$$
\frac{d^{2} \sigma}{d P_{\mathrm{T}} d y}=\sum_{i j} \int d x_{1} d x_{2} f_{i}\left(x_{1}\right) f_{j}\left(x_{2}\right) \frac{d^{2} \sigma(i j \rightarrow V)}{d P_{\mathrm{T}} d y}
$$

Figures 2.6 and 2.7 show several of the next-to-leading-order (NLO) production diagrams for $Z$ and $W$ bosons. Radiated quarks and gluons in these, as well as higher-order diagrams, contribute to the transverse momentum of the bosons [12]. For relatively high boson $p_{\mathrm{T}}\left(p_{\mathrm{T}} \sim M_{V}\right)$, 
the production cross section can be calculated perturbatively, and the resulting differential crosssection has the form:

$$
\frac{d^{2} \sigma}{d P_{\mathrm{T}} d y} \sim \alpha_{W} \alpha_{S}\left(a_{1}+a_{2} \alpha_{S}+a_{3} \alpha_{S}^{2}+\ldots\right)
$$

where $\alpha_{W}$ is the weak coupling constant and $\alpha_{S}$ is the strong coupling constant.

The dominant contributions to these higher order effects are of the form:

$$
\frac{d^{2} \sigma}{d P_{\mathrm{T}} d y} \sim \frac{\alpha_{W} \alpha_{S}}{p_{\mathrm{T}}^{2}} \ln \left(\frac{Q^{2}}{p_{\mathrm{T}}^{2}}\right)\left[v_{1}+v_{2} \alpha_{S} \ln ^{2}\left(\frac{Q^{2}}{p_{\mathrm{T}}^{2}}\right)+v_{3} \alpha_{S}^{2} \ln ^{4}\left(\frac{Q^{2}}{p_{\mathrm{T}}^{2}}\right)+\ldots\right]
$$

where $Q^{2}$ is the square of the boson mass. For low momenta $\left(p_{\mathrm{T}} \rightarrow 0\right)$, these perturbative calculations break down, because the $\frac{1}{p_{\mathrm{T}}^{2}} \ln ^{2}\left(\frac{Q^{2}}{p_{\mathrm{T}}^{2}}\right)$ terms diverge. In this non-perturbative regime, a method called "resummation" must be used to estimate the $p_{\mathrm{T}}$ spectrum [15].

Because the $W$ and $Z$ bosons share similar QCD production mechanisms and masses, the resulting $p_{\mathrm{T}}$ spectra are similar. We exploit this fact later, in our development of the Recoil Library Method.
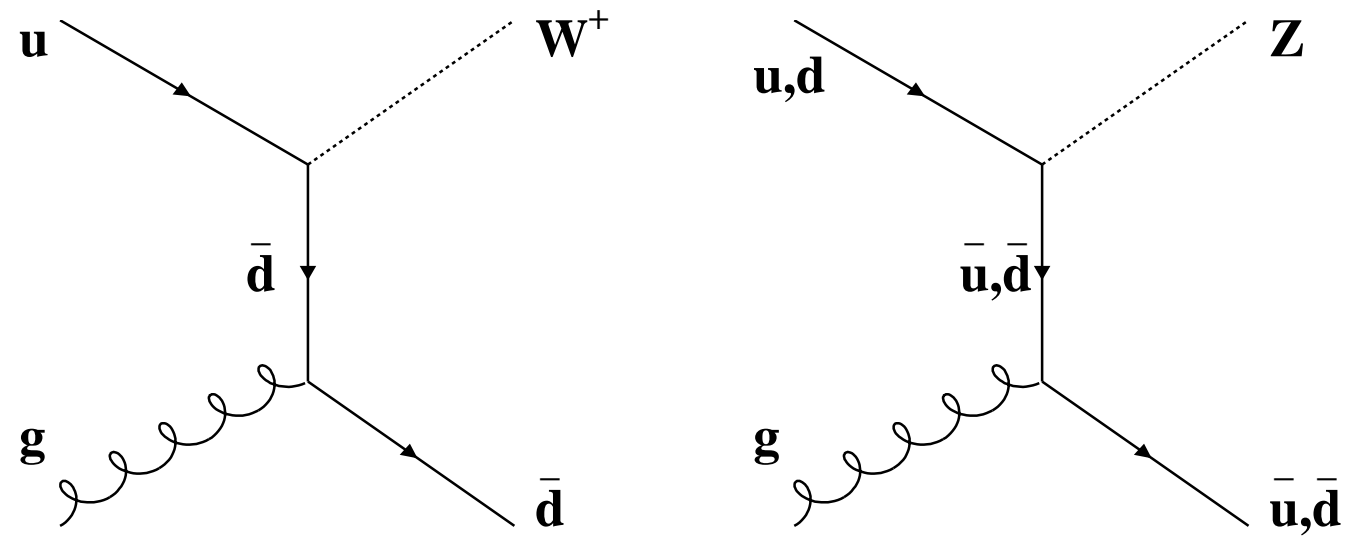

Figure 2.6: Compton scattering in $Z$ and $W$ boson production.

Figure 2.8 shows the known, leading-order decay channels of the $W$ and $Z$ bosons. In this 

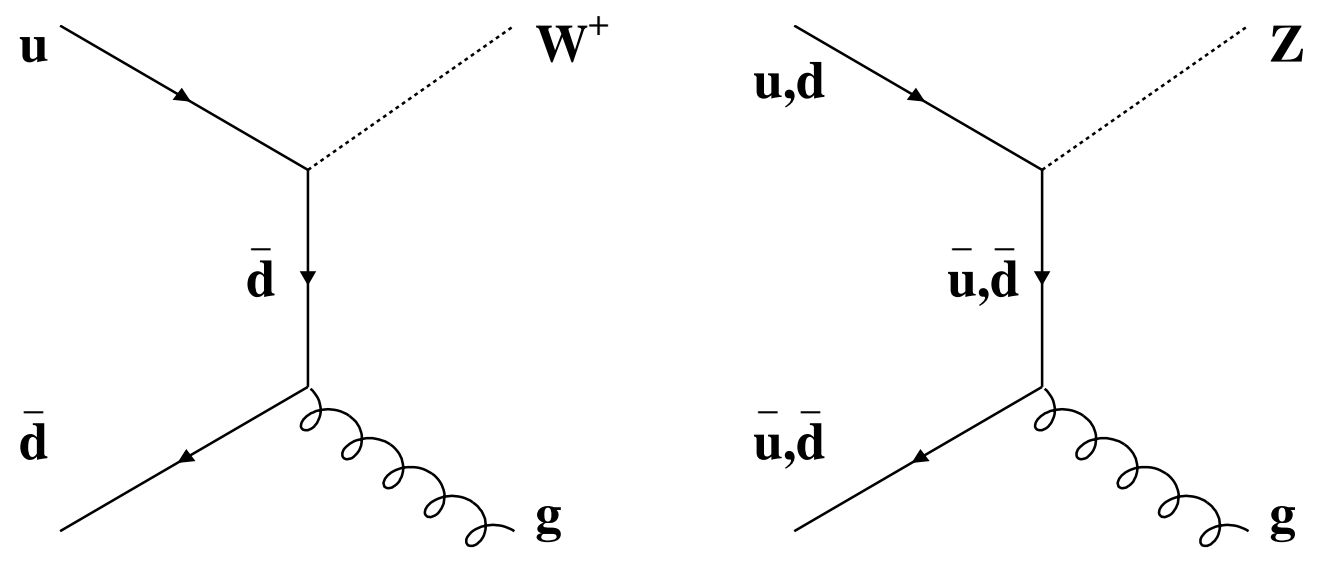

Figure 2.7: Initial-state gluon radiation in $Z$ and $W$ boson production.

thesis, we study the $W \rightarrow e \nu$ and $Z \rightarrow e e$ channels exclusively. These channels provide the cleanest signal to QCD backgrounds, and the D $\varnothing$ detector is best suited for measuring electron energies.

\subsection{The $W$ boson Mass and Width}

The $W$ and $Z$ boson masses are given by electroweak symmetry breaking as

$$
\mathrm{M}_{W}=g \nu / 2, \quad \mathrm{M}_{Z}=M_{W} / \cos \theta_{W}
$$

where $\nu$ is the vacuum expectation value of the Higgs field, $g$ is the electroweak coupling, and $\theta_{W}$ is the weak mixing angle. The tree-level $W$ boson mass is given by:

$$
M_{W}^{\text {tree }}=\left(\frac{\pi \alpha}{\sqrt{2} G_{F}}\right)^{1 / 2} \frac{1}{\sin \theta_{w}} \approx 79 G e V
$$

where $G_{F}$ is the Fermi coupling constant, which can be measured precisely from muon decays. Similarly, the tree-level $Z$ mass is roughly $89 \mathrm{GeV}$. The precise masses of these particles must include higher order diagrams, such as the Top quark and Higgs boson loops shown in Fig 2.10 and 2.9. The formula for the $W$ mass, including these effects can be given by: 


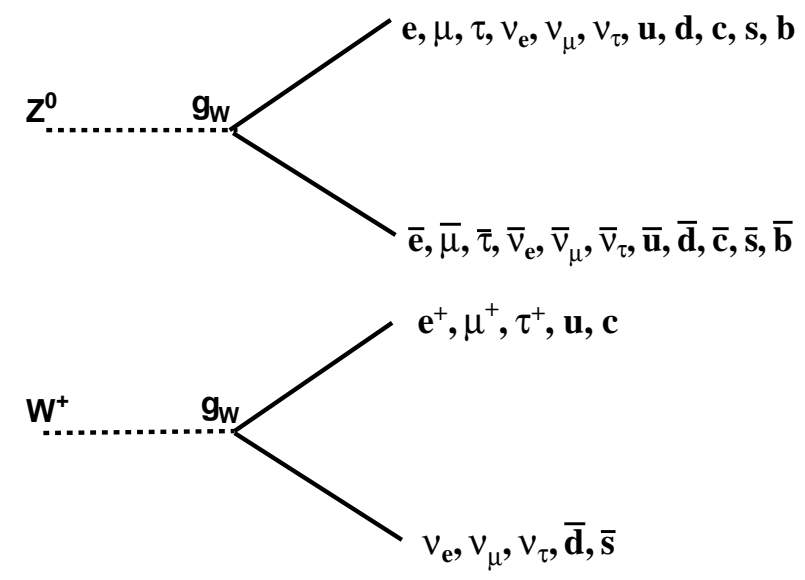

Figure 2.8: Known decay channels for the $W$ and $Z$ boson.

$$
M_{W}=\left(\frac{\pi \alpha}{\sqrt{2} G_{F}}\right)^{1 / 2} \frac{1}{\sin \theta_{w} \sqrt{1-\Delta r}}
$$

where $\Delta r$ are the radiative corrections from higher order effects [16], and account for a modification on the tree-level mass of a few percent. The correction to the $W$ boson mass $\Delta M_{W}=M_{W}-M_{W}^{t r e e}$ is related to the top quark mass and Higgs boson mass by $\Delta M_{W} \propto M_{\text {top }}^{2}-M_{\text {bottom }}^{2}$ and $\Delta M_{W} \propto$ $\log M_{H}$. From these relationships, we are able to compare the necessary precision of the $W$ boson mass measurement to that of the top quark mass in constraining the Higgs boson mass [17], given by $\Delta M_{W} \approx 0.006 \Delta M_{t}$. With the most precise single measurements of the top quark mass reaching uncertainties of around $1.6 \mathrm{GeV}$, we would need a precision on $M_{W}$ approaching $10 \mathrm{MeV}$ to achieve the equivalent constraint on the Higgs. This is the challenge for the combined $W$ boson mass measurements at $\mathrm{D} \varnothing$ and $\mathrm{CDF}$ in Run II of the Tevatron.

Additional contributions to $\Delta r$ can arise from physics beyond the Standard Model, such as SUSY. Thus, the $W$ boson mass serves both as a test of the Standard Model, and a probe for new physics.

The $W$ boson decay width $\left(\Gamma_{W}\right)$ is another significant standard model parameter $[18,19]$. Direct measurements of $\Gamma_{W}$ are important as complements to the indirect measurements extracted 


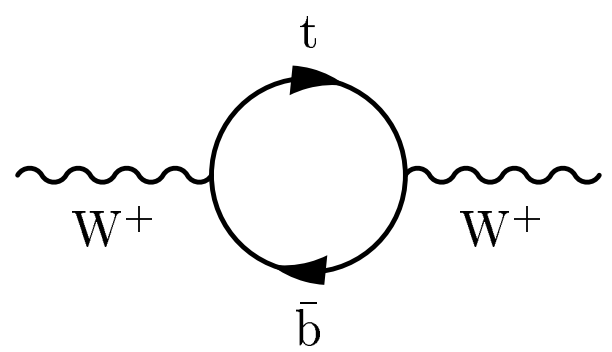

Figure 2.9: Higher order correction on the $W$ boson mass from a top-quark loop.

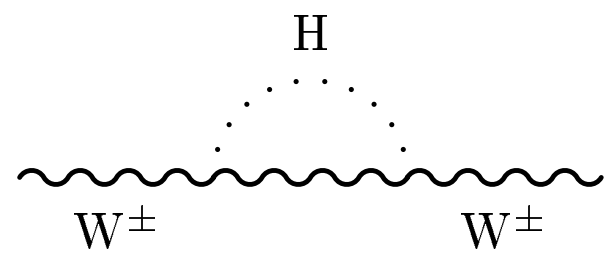

Figure 2.10: Higher order correction on the $W$ boson mass from a Higgs loop.

from the cross-section ratio $R$. The direct measurement has very different systematics from the indirect approach. Many of these systematics will scale down with more statistics in our calibration samples. Direct measurement of the $W$ boson width does not require theoretical inputs for $\sigma_{W} / \sigma_{Z}$ and $\Gamma(W \rightarrow e \nu)$ which might be sensitive to physics beyond the Standard Model. Because the width measurement looks at kinematic regions high above the mass pole, it is sensitive to new physics such as an additional heavy vector boson $\left(W^{\prime}\right)$. The partial width $\Gamma_{W}(W \rightarrow e \nu)$ is given by:

$$
\Gamma(W \rightarrow e \nu)=\frac{G_{\mu} M_{W}^{3}}{6 \pi \sqrt{2}}\left[1+\delta_{S M}\right]
$$

where $G_{\mu}=(1.16639 \pm 0.00002) \times 10^{-5} \mathrm{GeV} / \mathrm{c}^{2}$ is the muon decay constant, and $\delta_{S M}$ corresponds to small higher-order SM corrections.

The measurement described in this thesis assumes the Standard Model value for the ratio $\Gamma_{t o t}(W) / \Gamma(W \rightarrow e \nu)$, predicted to be

$$
\frac{\Gamma_{t o t}(W)}{\Gamma(W \rightarrow e \nu)}=3+6\left[1+\alpha_{s}\left(M_{W}\right) / \pi\right]
$$




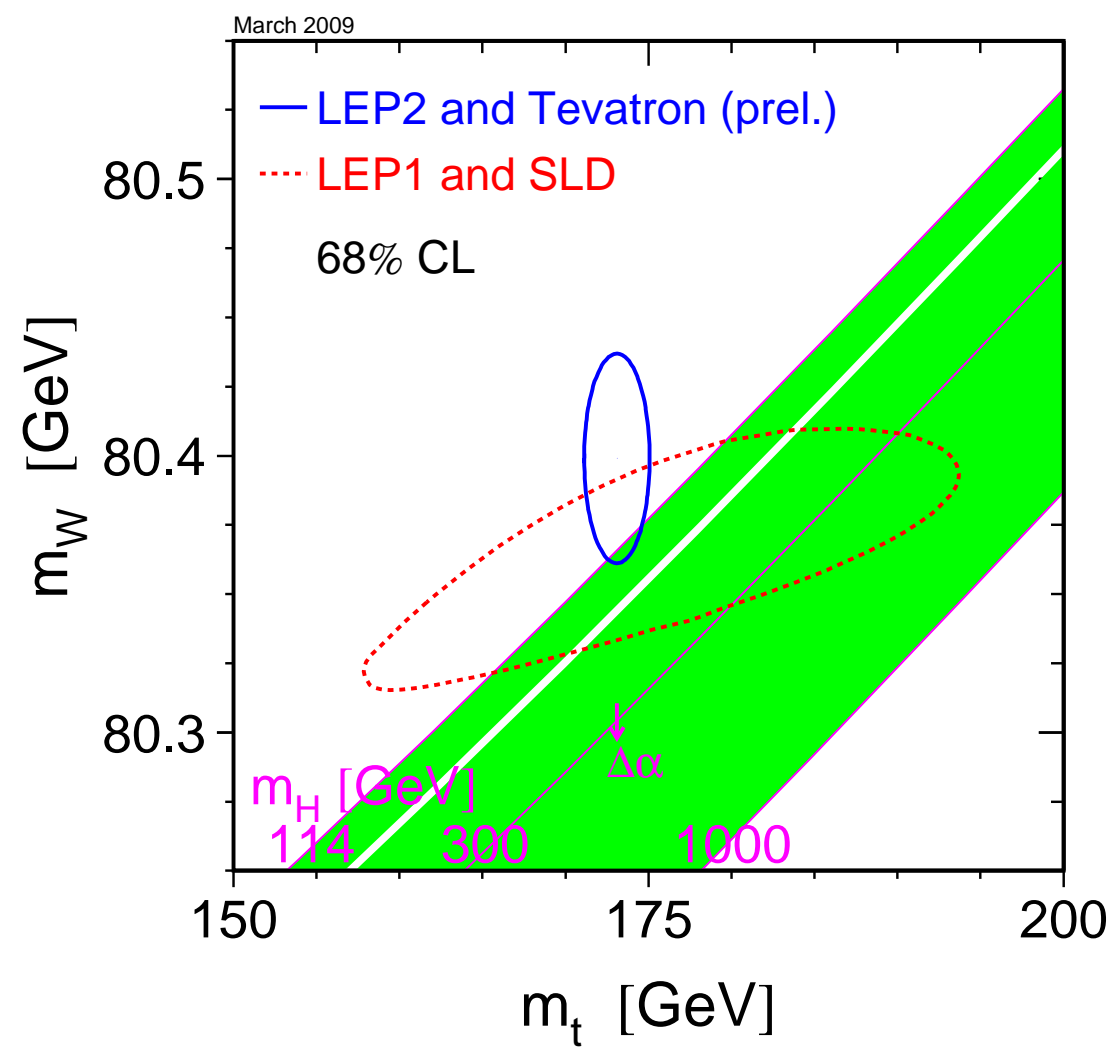

Figure 2.11: Constraints on the Higgs mass from $M_{W}$ and $M_{t o p}$. 
and is therefore sensitive to exotic decay channels that would raise this ratio [20]. Thus, $\Gamma_{W}$, like the mass, is a good probe for new physics. 


\section{Chapter 3}

\section{Measurement Strategy}

The $W$ boson mass and decay width are measured from data distributions of observables that are sensitive to $M_{W}$ and $\Gamma_{W}$. We compare the data distributions with simulated distributions generated at various mass or width values, until we find the mass or width that gives the closest comparison. A prerequisite for precision $W$ measurements is an accurate, fast MC method for producing these template distributions. This fast simulation must reproduce the underlying physics for the relevant processes. It must also accurately reflect the detector response for measuring the observables. Both the lepton energy and the measured recoil need to be well described by the fast MC in order to accurately extract the $W$ mass and width. For further reading, see [21]-[25] on the $W$ boson mass, and [26]-[28] on the direct width measurement. In this chapter we look at the kinematic observables used to extract $M_{W}$ and $\Gamma_{W}$. We describe the overall strategy for producing the template distributions that we compare with data, and the fit method used to extract the mass and width from these template fits.

\subsection{Kinematics and Observables}

In typical hadron colliders like the Tevatron, only the leptonic $W$ boson decay channel $(W \rightarrow e \nu, l=\mathrm{e}, \mu)$ provides a sufficiently clean detector signature over QCD backgrounds. For similar reasons, the $Z$ data used in our analyses are $Z \rightarrow l l$ decays. In this dissertation we focus on the electron channel, specifically.

Since neutrinos in $W \rightarrow e \nu$ decays cannot be directly measured, their energy must be inferred using momentum conservation. Since typical hadron collider detectors are uninstrumented in the forward region along the beam pipe $(|\eta|>\sim 4.0)$, some of the forward momentum is lost, and we can only evoke conservation of momentum in the transverse plane with respect to the beam direction. Typical observables include the transverse momentum of the electron $\left(p_{\mathrm{T}}(\mathrm{e})\right)$, missing 
transverse energy $\left(E_{T}\right)$, and a kinematic quantity known as the transverse mass $\left(M_{\mathrm{T}}\right)$, given by:

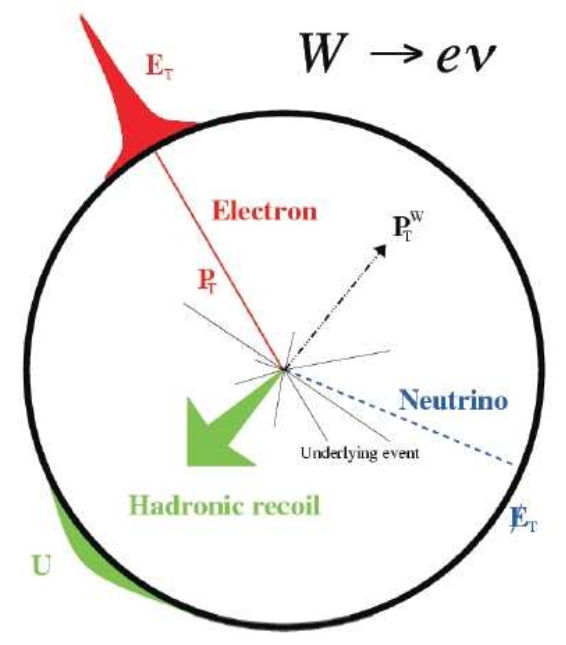

Figure 3.1: Anatomy of the transverse energy in the $D$ detector in a typical $W \rightarrow e \nu$ event.

$$
M_{\mathrm{T}}=\sqrt{2 p_{\mathrm{T}}(e) E_{T}(1-\cos (\Delta \phi))},
$$

where $\Delta \phi$ is the opening angle between the charged lepton and $E_{T}$ in the transverse plane. This resembles the form for the invariant mass:

$$
M=\sqrt{2 p\left(e_{1}\right) p\left(e_{2}\right)(1-\cos (\Delta \Omega))},
$$

Figure 3.2 shows typical $M_{\mathrm{T}}$ and $p_{\mathrm{T}}(e)$ distributions for a $W$ boson. The $E_{T}$ distribution looks similar to the electron $p_{\mathrm{T}}(e)$. The key features of these distributions are the sharp "Jacobian Edge" followed by a long tail in the high $M_{\mathrm{T}}$ or $p_{\mathrm{T}}$ regions. These regions are most sensitive to the underlying physics of the $W$ boson, whereas the broad shoulder in the low $M_{\mathrm{T}}$ or $p_{\mathrm{T}}$ regions is mostly driven by kinematics and selection efficiencies. The Jacobian Edge for all three observables is most sensitive to $M_{W}$, whereas the fraction of events in the tail is most sensitive to the natural width, $\Gamma_{W}$.

$W$ boson mass measurements extracted from the $M_{\mathrm{T}}, p_{\mathrm{T}}(e)$, and $E_{T}$ distributions have different systematic sensitivities, and thus complement each other. One can see from Fig. 3.2, 
that the $M_{\mathrm{T}}$ distribution is very robust over wide variations in the input $p_{\mathrm{T}}^{W}$ spectrum, whereas the $p_{\mathrm{T}}(e)$ spectrum varies significantly for different input boson momenta. This makes conceptual sense, since the effects of a boost on the electron and $E_{T}$ should cancel in the calculation $M_{\mathrm{T}}$, while the $p_{\mathrm{T}}(e)$ spectrum is explicitly dependent on the momentum of the decaying boson. In contrast, we see that the $M_{\mathrm{T}}$ observable is sensitive to detector resolution effects, which the $p_{\mathrm{T}}(e)$ is relatively stable.
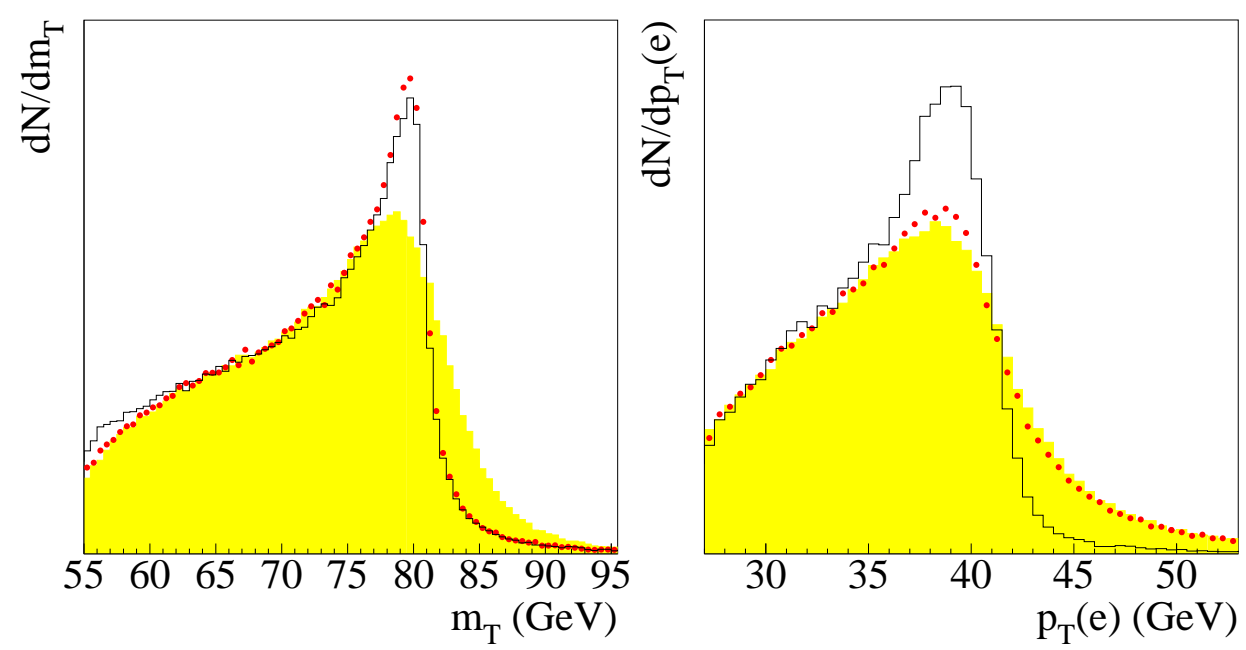

Figure 3.2: $M_{\mathrm{T}}$ (left) and $p_{\mathrm{T}}(e)$ (right) spectra for $W$ bosons with $p_{\mathrm{T}}^{W}=0$ (solid line), with the correct $p_{\mathrm{T}}^{W}$ spectrum (points), and with detector resolutions (shaded area).

We identify $W \rightarrow l \nu$ decays by selecting events with one high transverse energy lepton and large $E_{T}$, corresponding to the neutrino. Conceptually the $E_{T}$ is reconstructed by vectorially summing the lepton transverse momentum and the "measured recoil" (or "recoil system") which comprises all of the additional transverse momentum in the detector besides the lepton.

The recoil system consists of the hard recoil from gluon or quark radiation and underlying event, as well as energy from additional collisions, "pileup" (which we define as residual energy from previous crossings), overlap with electron energy, and noise.

The hadronic recoil is difficult to model on first principles because it is not a singular object, like the decay lepton. The various components of this measured recoil system all have different dependences on instantaneous luminosity. For example, pile-up and additional $p \bar{p}$ interactions scale 
with instantaneous luminosity, while the underlying event is luminosity independent. Moreover, detector effects such as zero-suppression cuts can introduce correlations between the calorimeter response to the hard recoil and any additional hadronic energy in the event. Because of this crosstalk, the hard recoil and various soft recoil components cannot be treated as independent effects in the detector.

Kinematically, the true momentum of a boson and that of its hard recoil should be equal and opposite. Because the detector response to hadronic energy is very different than the response to lepton energy $(e / h \approx 1 / 2)$, the measured recoil and measured boson momentum are not typically the same. Consequently, the recoil is relatively poor measure of $p_{\mathrm{T}}^{W}$. However, the $p_{\mathrm{T}}$ of the $Z$ boson can be reconstructed as the sum of the momenta of the two decay leptons. Since lepton energies are well measured and well understood in collider detectors, the dilepton $p_{\mathrm{T}}$ provides a good first approximation of the true boson momentum.

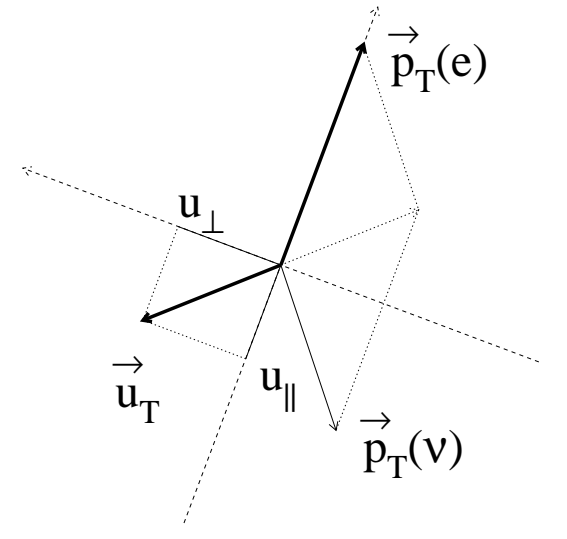

Figure 3.3: Definition of $u_{\|}$and $u_{\perp}$. It should be noted that $u_{\|}$is defined as negative when opposite the electron direction.

For the $W$ boson, we can only approximate the boson momentum using the recoil, since the momentum of the neutrino is lost. We often refer to the recoil $p_{\mathrm{T}}$ of the event as $u_{\mathrm{T}}$.

Two other useful kinematic quantities used in our efficiency model and to check the accuracy of our recoil model are $u_{\|}$and $u_{\perp}$, which are defined as the parallel and perpendicular components of the recoil with respect to the electron direction in the transverse plane. Figure 3.3 illustrates 
this definition.

\subsection{Template Production}

We use a fast Monte Carlo (MC) model to generate template distributions of various $W$ boson observables at varying mass and width values. This fast Monte Carlo Model must accurately simulate the underlying physics for the production and decay of $W$ bosons, and detector effects.

For testing and cross-check purposes we have two different fast Monte Carlo methods, built around a common event generator and parametric model for the electron measurement, but with different recoil models. In this section we describe the main common elements of both fast MC models.

\subsubsection{Generator}

We use two event generator configurations as inputs to our fast Monte Carlo Model, PYTHIA, and a combination Resbos [29] and Photos [30]. The combined REsbos + PHOTOs is used for our main data analysis. PYTHIA [31] is used in our studies from a GEANT-based detector model (described at the end of this section), because it has a hadronization model that describes the complete event, even though its boson $p_{\mathrm{T}}$ model is known to be inadequate. In this thesis, we will focus mainly on the event generation used in the data analysis.

RESBOS, which stands for RESummed BOSon production and decay, computes the differential cross-section for production of $W$ and $Z$ bosons in $p \bar{p}$ collision, including soft-gluon, resummed QCD corrections. At large $p_{\mathrm{T}}$, where resummation techniques fail, RESBOs uses a fixed, nextto-leading order, perturbative calculation. Рнотоs is used to model final state radiation (FSR). Recent D $\varnothing$ analyses have found that this RESBOS + PHOTOS combination is successful in accurately describing boson $p_{\mathrm{T}}$ spectra [32]. The inputs to this generator and their uncertainties will be discussed further in Chapter 12. 


\subsubsection{Detector Simulation}

The two main components of the detector simulation are the electron measurement and the recoil measurement. Our electron model is parametric, whereas the main recoil model used in this thesis is the "Recoil Library Method", which extracts recoils from a library of data or MC $Z \rightarrow e e$ recoils . There is also a parameterized recoil model used for the default $W$ boson mass analysis ("Parameterized Recoil Method"), but we use that model only as a cross-check on the Recoil Library Method in this thesis. Both the electron model and recoil simulations will be described at length in Chapters 7 and 9. In addition to recoil and electron simulations we apply efficiency models (Chapter 8) and backgrounds (Chapter 10). Adjustable parameters in the fast MC are tuned to an independent but kinematically similar data set, namely the $Z \rightarrow e e$ sample. The recoil library used in the Recoil Library Method also comes from this sample. Accordingly, many of the systematic uncertainties in the $W$ boson mass and width measurements are limited by the precision of our tuning to the roughly $18 \mathrm{k}$ central $Z \rightarrow e e$ events we have available.

We generate fast MC in steps of different mass or width values for the template sets used in our fits. Rather than entirely regenerating our fast MC for each different mass and width value, we generate our fast Monte Carlo at the nominal mass value and width in REsBos, and rewieght the events by the probability that each generated event at the nominal mass and width could occur at the various mass and width values of the templates. This reweighting factor is determined by the Breit-Wigner Equation, and allows us to make all of our templates analytically from one set of simulated events.

\subsubsection{Testing}

To test our fast MC methods and asses systematic uncertainties, we often generate "toy" data or "psuedo experiments" using the same fast MC model that we used to make the template distributions for our fits. We measure the $M_{W}$ or $\Gamma_{W}$ in these toy samples to study statistical effects in our measurement procedure or systematic effects due to variations in the tunable MC parameters. We test for biases in the implementation of the Recoil Library Method on "toy" $W$ 
data and $Z$ recoil libraries, made from samples created using the Parameterized Recoil Method, and extracting the $\mathrm{W}$ mass and width from these fast $\mathrm{MC}$ samples.

We also test both the Recoil Library Method and the Parameterized Method on a more realistic MC sample produced using a GEANT based full detector model with full offline reconstruction as in data. The full simulation uses PYTHIA to simulate the production and decay of the W boson, as well as the underlying event and any final state radiation. The four-vectors are then propagated through an accurate material description of the detector using GEANT. GEANT is a detector description and simulation tool that propagates generated high energy particles through an accurate material description of the detector, calculating the probabilities of atomic and nuclear interactions, based on their well-known cross sections for each step of the particles' paths [33]. Real zero-bias data with the same instantaneous luminosity profile as the $W \rightarrow e \nu$ sample is overlaid on the full detector simulation to model additional collisions and noise in the detector. The raw signal is then processed and reconstructed as it would be in data. This full detector simulation will be referred to as "full MC" for the rest of this thesis.

\subsection{Basic Fit Strategy}

The $W$ boson mass is extracted from fits to the template sets of $M_{\mathrm{T}}, p_{\mathrm{T}}(e)$, and $E_{T}$ distributions using a binned negative log-likelihood method [34] and a custom made utility called wzfitter [35], built around the MINUIT minimization package [36]. High statistics template distributions are generated using the model described in this dissertation for different values of the $W$ Mass in $10 \mathrm{MeV}$ steps. Polynomial splining is used to interpolate between those steps and generate $W \rightarrow l \nu$ templates for a continuum of mass values. This is an appropriate approach to interpolation, since all of the template distributions are made from the same, single set of generated $\mathrm{W}$ events (re-weighted according the the Breit-Wigner distribution) and thus vary smoothly. We compare the data to these simulated distributions for varying mass values, until we find the $W$ mass which minimizes the negative log-likelihood. Our fit ranges are $[32,48] \mathrm{GeV}$ for the $p_{\mathrm{T}}(e)$ and $E_{T}$ distributions, and $[60,90]$ for the $M_{\mathrm{T}}$ distribution. For the $W$ boson width we fit the $M_{\mathrm{T}}$ 
distribution over the range $[100,200] \mathrm{GeV}$.

We approach normalization differently for the $\mathrm{W}$ mass and $\mathrm{W}$ width measurements, since we are looking at different subranges of the relevant observables. For the $\mathrm{W}$ width fit, we normalize up to but excluding the tail region, and then fit over the tail region of the transverse mass distribution. For the $\mathrm{W}$ mass measurement, we normalize over the same subrange that we fit, regardless of whether we use $M_{\mathrm{T}}, p_{\mathrm{T}}(e)$, or $E_{T}$ as our observables.

For the fits to the various $W$ and $Z$ boson observables, we almost exclusively use the negative log-likelihood, given by the following formula:

$$
L=\sum_{i=0}^{N}-d_{i} \log t_{i}+t_{i}
$$

Where $i=0$ is the first bin of the histogram, $N$ is the total number of bins, including the overflow, $d_{i}$ is the content of the $i$ th bin in data, and $t_{i}$ is the content of the ith bin in the template distribution we are comparing with the data.

\subsubsection{Blinding}

One other important feature is also built into the wzfitter code, which is used in the data analysis only. The data analysis described in this thesis is a "blind analysis" [37]. The actual $M_{W}$ or $\Gamma_{W}$ value of the fit is hidden from the analyzers, to prevent any biased predisposition to adjust the model based on the expected PDG $W$ boson mass or width. Instead, the fitter provides us the fit values with a hidden offset added on. We cannot know the true fit results to $M_{W}$ or $\Gamma_{W}$, but we can still test the consistency of the fits over different fit ranges and sub-sets of the data, which should hopefully give the same blinded result. We have also incorporated the blinding into our plotting software, so we can compare data with fast MC generated at the actual fit value, given the blinded value. Once these various cross-checks are performed and the analyzers are confident in there results, the blinding is turned off and the final fits values revealed. 


\section{Chapter 4}

\section{Experimental Setup}

\subsection{The FermiLab Accelerator}

The accelerator at Fermilab is a $p \bar{p}$ collider with $1.96 \mathrm{TeV}$ center of mass energy, the most luminous hadron accelerator and highest energy collider of any kind until the turn-on of the LHC at CERN [39]. The main ring of the accelerator, where protons and anti-protons reach their maximum energies of $980 \mathrm{GeV}$, is the $6.28 \mathrm{~km}$ circumference Tevatron. However, the Tevatron is actually the end of a multi-part chain of accelerators, storage rings, and switch-points used to produce and accelerate the hadrons. This finely tuned system is run day and night by hundreds of engineers who monitor and adjust its roughly 200,000 control parameters. Figure 4.1 illustrates the the Fermilab accelerator.

Protons are generated from a source of $H^{-}$ions accelerated initially by a Cockroft-Walton generator and, then, a linear accelerator known as the Linac. In the Linac the ions reach an energy of $400 \mathrm{MeV}$ before passing through a thin carbon foil, which strips off the electrons, leaving a pure proton beam. The protons are stored at $8 \mathrm{GeV}$ and organized into a bunch structure in the Booster ring before being passed to the Main Injector. Anti-protons are produced by colliding $120 \mathrm{GeV}$ protons from the Main Injector into a nickel fixed target. Roughly 1 anti-proton is generated for every $10^{5}$ protons. The anti-protons are collected and then cooled to $8 \mathrm{GeV}$, stored, and organized into a bunch structure in the Debuncher and Accumulator Ring.

Both protons and anti-protons are accelerated to $150 \mathrm{GeV}$ in the Main Injector before entering the Tevatron to reach the final $980 \mathrm{GeV}$. These charged particles are accelerated by alternating electric fields and held in their circular path by super-conducting magnets. The beam of protons and anti-protons is organized into 36 bunches. These 36 bunches are divided into 3 super-bunches, of 12 bunches each. The bunches within each super-bunch are separated by 


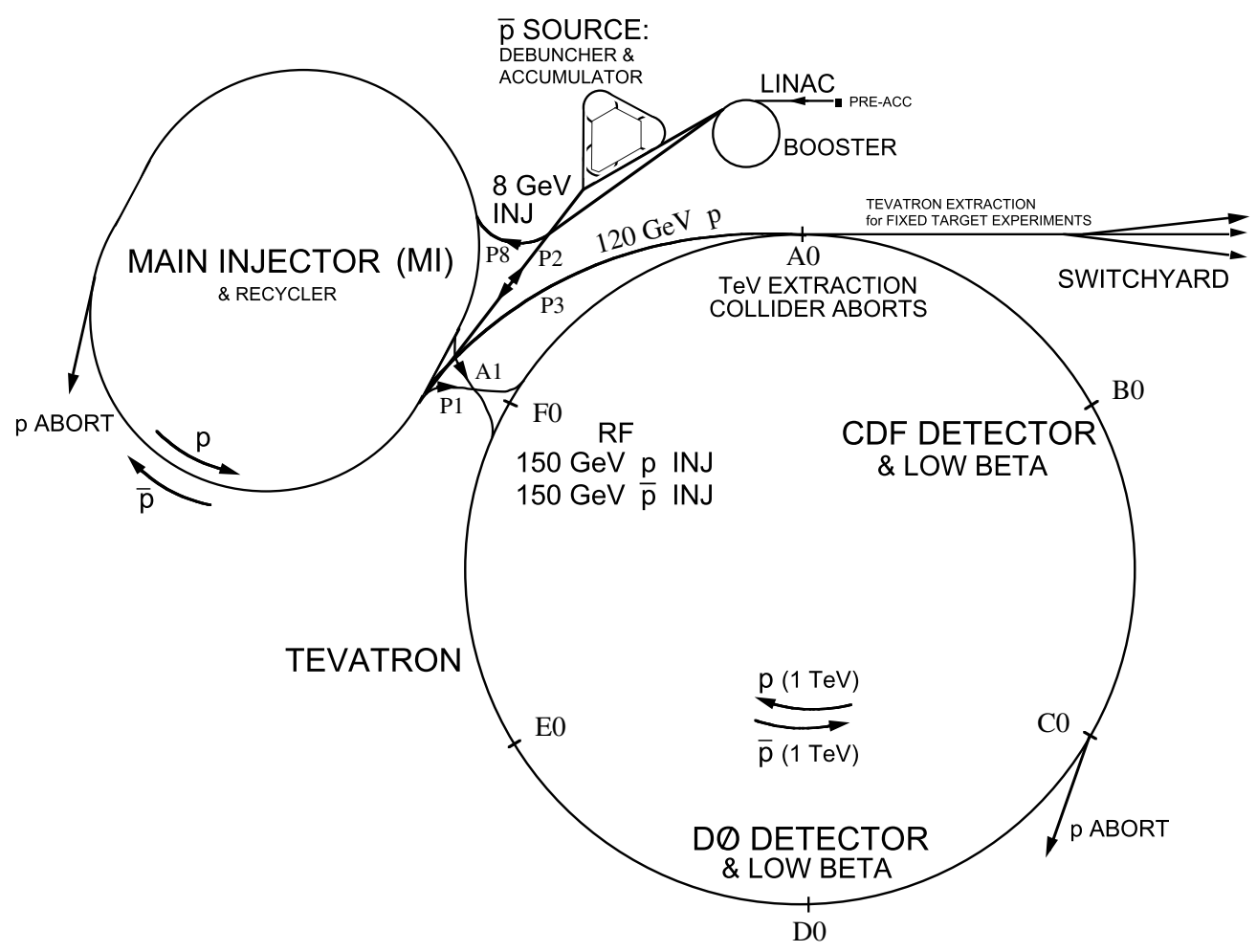

Figure 4.1: The structure of the Fermilab collider.

3, 132ns "ticks" and the super-bunches are each separated by 17 such ticks. This structure is illustrated in Fig 4.2.

The proton and anti-proton beam circle in opposite directions, separated from each other along helical trajectories. The beams are compressed by focusing quadrapole magnets and collide at two points along the Tevatron, $\mathrm{B} 0$ where $\mathrm{CDF}$ is located, and $\mathrm{D} \varnothing$. The cross section of the beam at these collision points is roughly $10^{-5} \mathrm{~cm}^{2}$, and the length of the luminous region is roughly 30 $\mathrm{cm}$.

\subsection{The DØ Detector}

The $\mathrm{D} \varnothing$ detector, shown in Fig 4.4 is a multipurpose array of subdetectors, each designed to measure different properties of the high energy particles produced in $p \bar{p}$ collisions. The main components are the tracking system used for position and momentum measurement of charged 


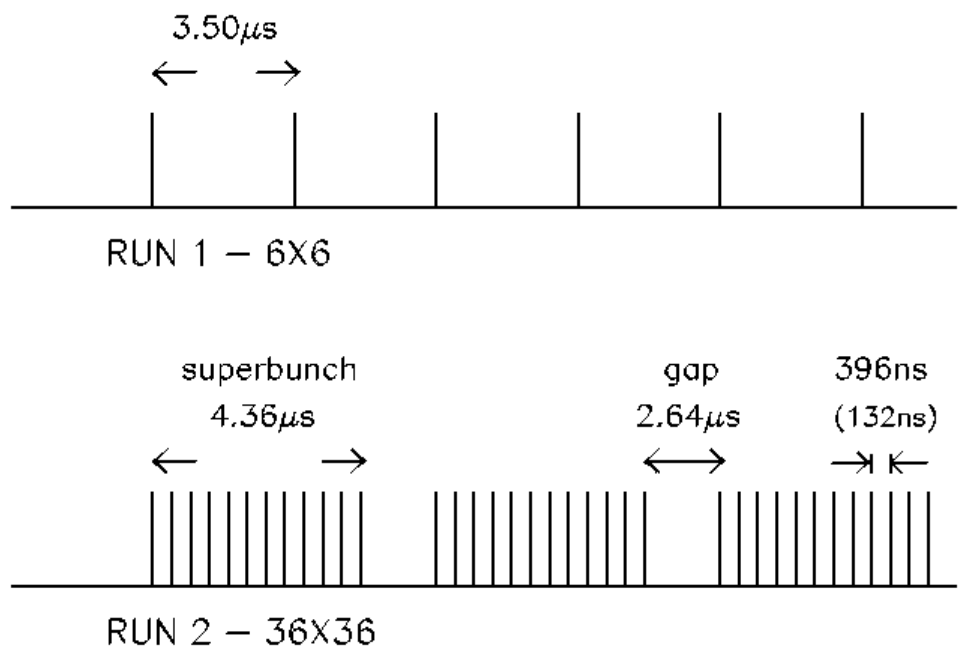

Figure 4.2: The Tevatron bunch structure for Run II

particles, the pre-shower detectors and calorimeters used to measure energy, the muon system for identification and momentum measurement of muons, the luminosity counters, and trigger system. The detector follows a cylindrical geometry, wrapped around the beam axis, which we define as the z-axis. The coordinate system is shown in Fig 4.3. More detailed detector descriptions can be found in $[40,41,42]$.

Because the beam pipe through the $\mathrm{z}$ axis of the detector is left un-instrumented, particles with high forward or backward momentum can escape undetected. As a consequence of this, the full energy and momentum of a collisions cannot be measured and we are forced to use transverse observables, as was discussed in Chapter 3.

\subsubsection{Tracking System}

The tracking system, used for precision position and momentum measurements, consists of three primary components: a silicon microstrip detector (SMT) [43] a central fiber tracker (CFT) [44], both surrounded by a 2 Tesla solenoid magnet. Figure 4.5 shows the complete tracking system.

The solenoid is a superconducting magnet, consisting of coiled niobium-titanium wire and 


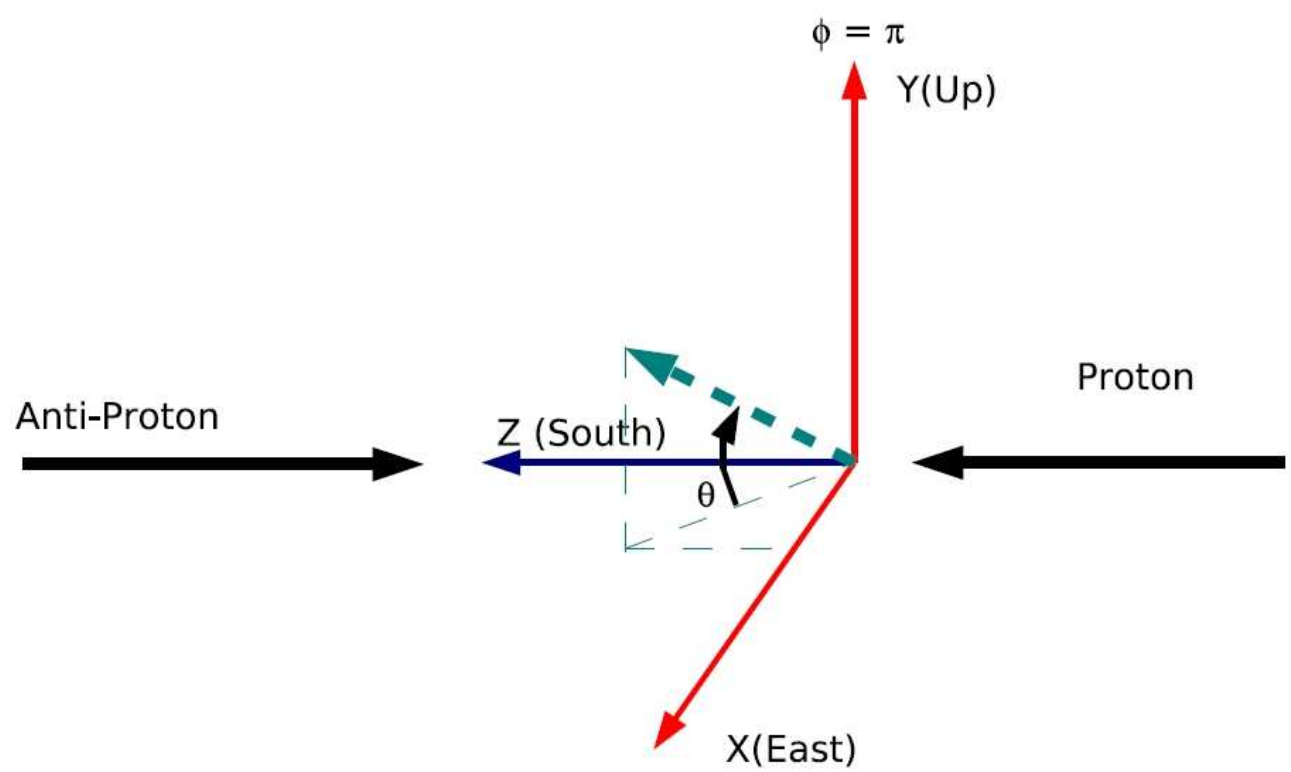

Figure 4.3: The D0 coordinate system

encased in an aluminum cylinder. The magnet is chilled to 4.2 Kelvin with liquid helium. The solenoid is 2.8 meters in length, $1.42 \mathrm{~m}$ in diameter and has an average thickness of $60 \mathrm{~cm}$. The solenoid and cryostat wall together contribute around 1.0 radiation lengths of material in front of the calorimeter.

Charged particles bend in the magnetic field provided by the solenoid. This curvature is measured by the SMT and CFT and used to determine the transverse momentum per charge $p_{T} / \mathrm{q}$ according to the relationship:

$$
\frac{p_{\mathrm{T}}}{q}=R B
$$

Where $\mathrm{R}$ is the radius of curvature and $\mathrm{B}$ is the magnetic field.

\subsubsection{The Silicon Microstrip Tracker}

The silicon microstrip detector, shown in Fig 4.6 is the first detector encountered by particles produced in the Tevatron collisions. It consists of 6 concentric barrels, $12 \mathrm{~cm}$ in length, with 16 interspersed disks (12 F-disks and $4 \mathrm{H}$-disks) normal to the beam line. Generally, the barrel 


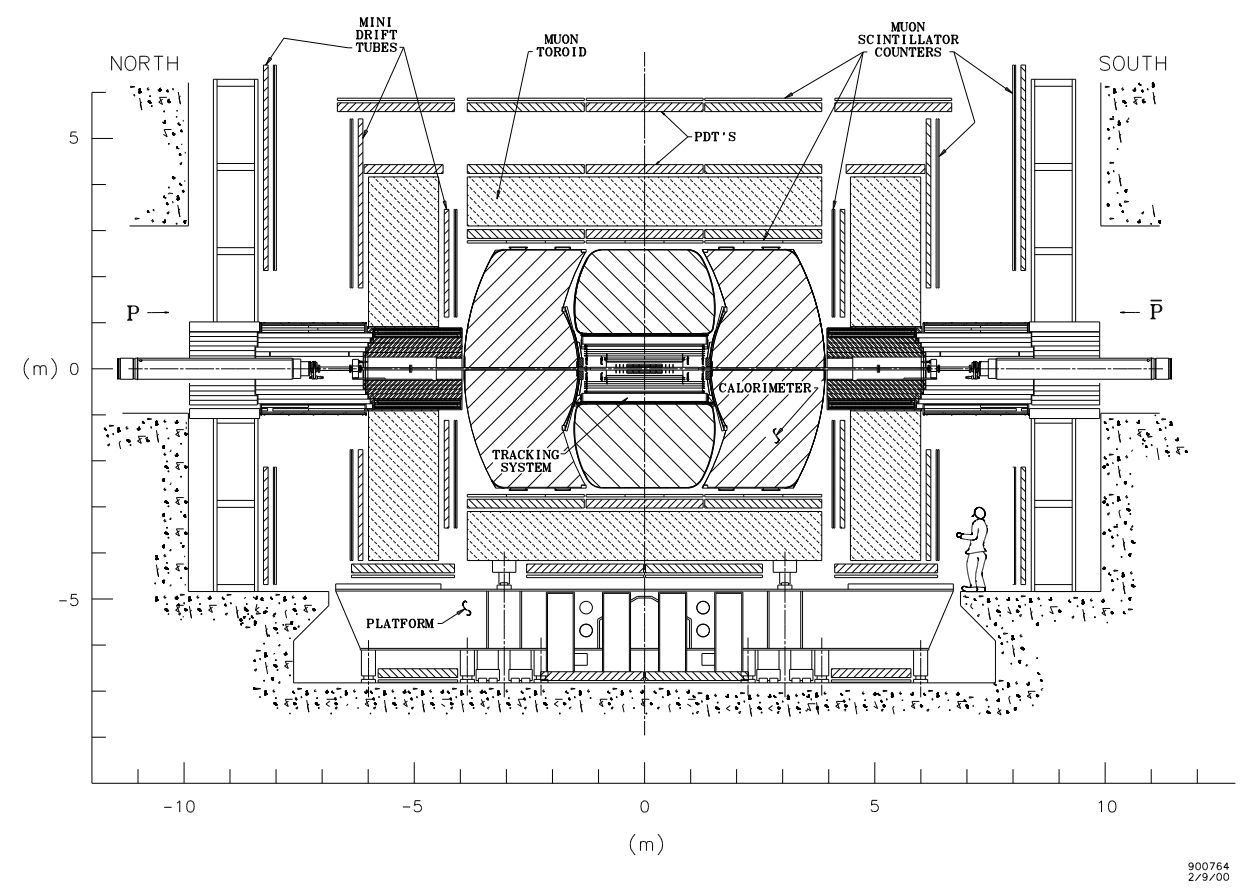

Figure 4.4: The D0 detector system. 


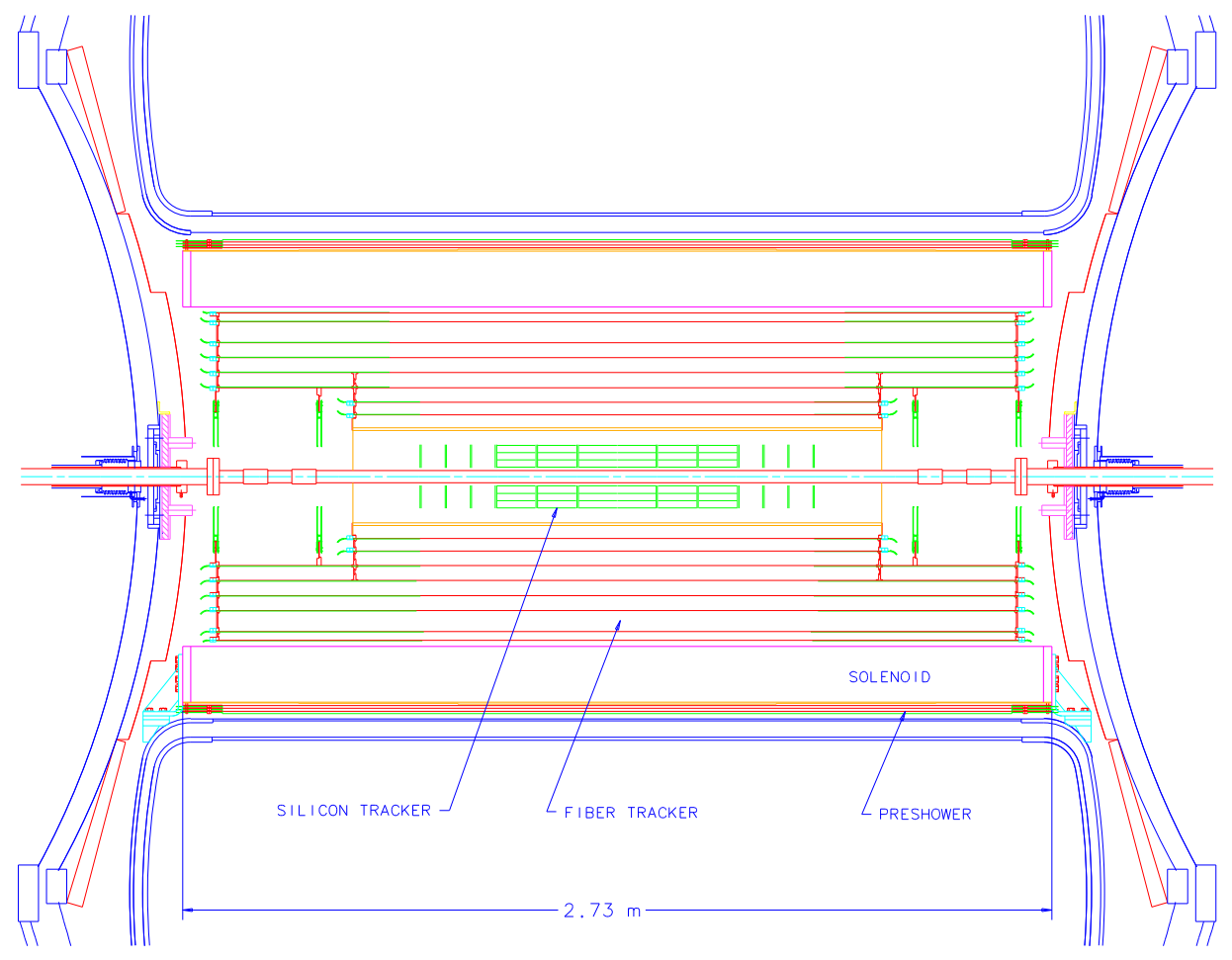

Figure 4.5: A side-view of the complete tracking system 
provides good tracking measurements for transverse particles, whereas the disks are used to measure the tracks very forward particles.

Silicon tracking systems typically work by measuring the trail of ions left by high energy charged-particles passing through sheets of silicon, collected on metallic strip contacts. The charge distribution on these strips provides position resolution in the direction perpendicular to the strips. By using two, alternating and orthogonal strip directions, spaced at various radial distances from the beam (in the case of the barrel) or positions in the z-direction along the beam line (in the case of the disks), we are able to reconstruct a three dimensional track. The D $\varnothing$ SMT has approximately 793,000 such readout channels, and provides an $r-\phi$ hit resolution of roughly $10 \mu \mathrm{m}$.

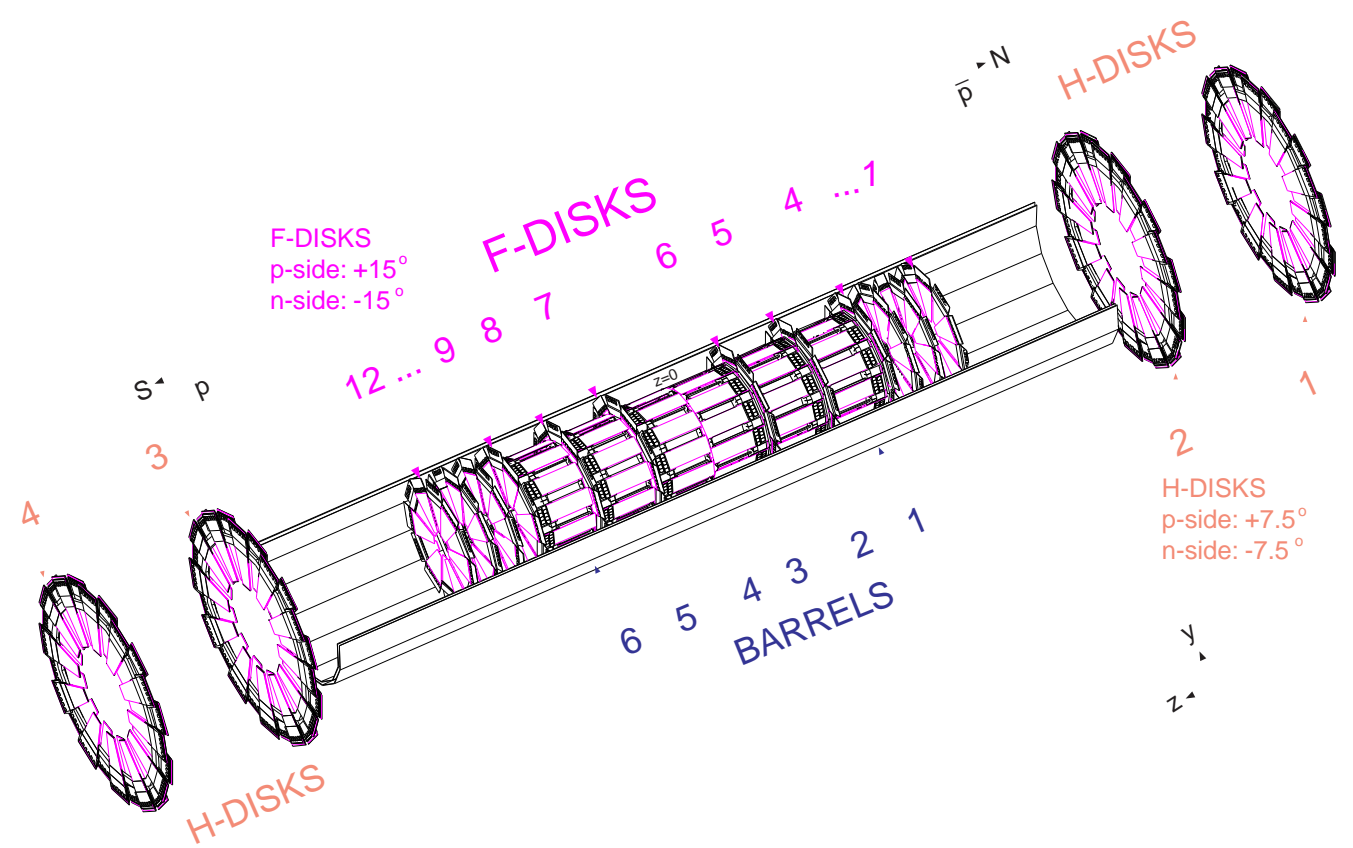

Figure 4.6: The D0 Silicon Microstrip Tracking detector.

\subsubsection{The Central Fiber Tracker}

Surrounding the SMT (Fig. 4.7) is the DØ Fiber tracking system, which extends radially 20 to $52 \mathrm{~cm}$ from the beam pipe and covers up to $|\eta|<1.7$. The SMT detector consists of two layers of scintillating fibers, one set along the axial direction to provide the $\phi$ coordinate, and the outer 
layer consists of two layers of "stereo" fibers set at \pm 3 degrees to help resolve the $\eta$ coordinate of a track. Each ionizing particle produces around 10 photons, on average, which are then detected by a Visible Light Photon Counter (VLPC) which converts the light into an electrical signal.

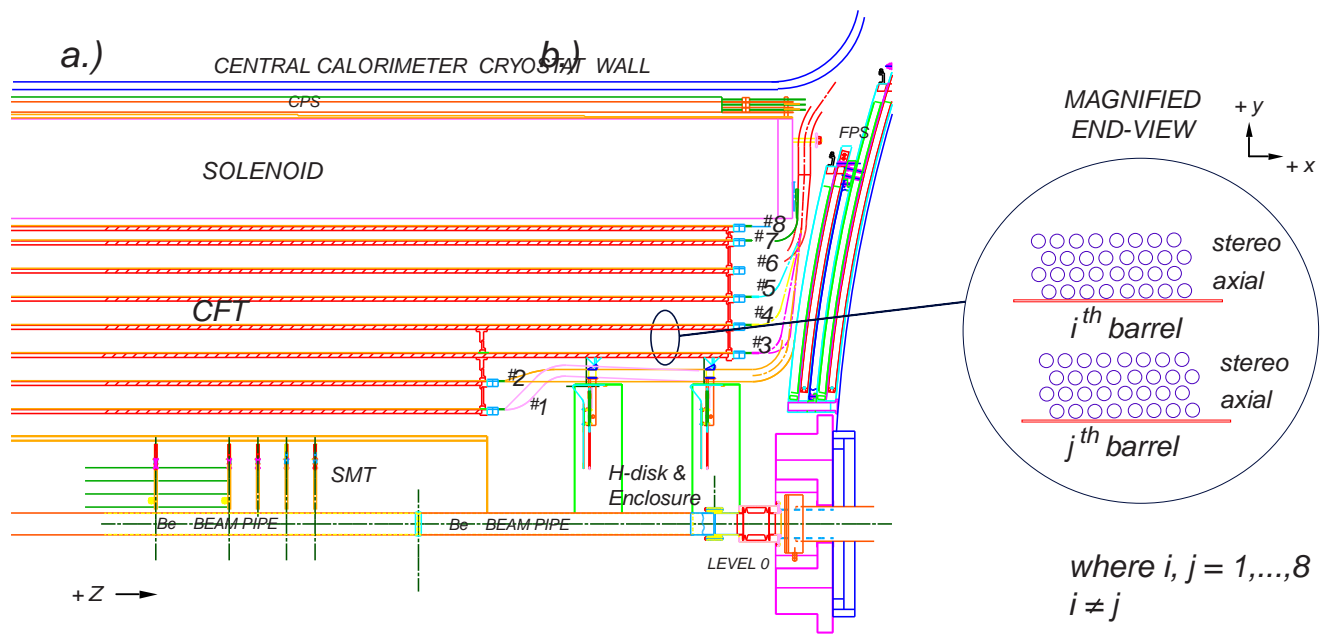

Figure 4.7: A side-view of the D0 Central Fiber Tracking detector.

\subsubsection{Preshower Detectors}

With a great deal more material in front of the calorimeter in Run II, a new detector subsystem was introduced, just in front of the calorimeter, to recover some of the shower energy before the cryostat wall. The pre-shower detectors consist of lead radiator, $1 X_{0}$ thick at normal incidence, and triangular scintillator strips to sample the shower energy. The central preshower (CPS) [45] covers $|\eta|<1.3$ and the two forward preshowers (FPS) [46] cover $|\eta|<2.5$. Unfortunately, signals delivered to the electronics for this detector subsystem saturated during the Run IIa period over which the data for this analysis was collected [47]. Consequently, the preshower is not used in this dissertation.

\subsubsection{Calorimeter}

The D0 calorimeter is a sampling calorimeter [48]. Thick plates of Uranium alternate with active layers of liquid argon, containing signal boards to collect charge. Energetic particles passing 
through materials tend to produce cascading showers of secondary particles. In the calorimeter, the formation of these showers is driven by the dense, uninstrumented Uranium layers, while the active layers of liquid argon measure discrete "samples" of the showers at various stages of their development. We calibrate the calorimeter so that we can recover a measurement of a given particle's total energy from the portion of that energy sampled in the liquid argon. We can use Monte Carlo simulations of shower development, along with test beam data and in-situ $Z \rightarrow e e$ data, to determine the appropriate layer weights and proportionality constants necessary to perform this conversion from layer signals to proper energy $[50,51]$.

A typical unit cell of the calorimeter, shown in Fig 4.10, consists of a alternating layers of absorber material and signal boards separated by a $23 \mathrm{~mm}$ liquid argon gap. Charged particles produced from the showering in the Uranium drift through the liquid argon to the signal boards, kept at $2.0 \mathrm{kV}$ with respect to the grounded absorber plated. The sizes of the unit cells are designed to compare with the transverse size of showers, $\Delta \mathrm{R} \sim 0.2$ for electromagnetic showers and $\Delta \mathrm{R} \sim 0.5$ for hadronic showers.

There are three distinct calorimeters, each housed in a separate cryostat. The central calorimeter or CC, which covers $|\eta|<1.1$, and two "end-cap" calorimeters for the forward region $1.5<|\eta|<4.2$. An "inter cryostat detector" (ICD) is located in the two massless gaps between the three calorimeters, covering $1.1<|\eta|<1.4$, to recover shower energy that would otherwise be lost in that region. In this analysis, we exclusively use central electrons. The $E_{T}$ is measured using both the CC and EC calorimeters, and the ICD is not used.

Longitudinally, the calorimeter is divided into three regions: electromagnetic (EM), fine hadronic $(\mathrm{FH})$, and course hadronic $(\mathrm{CH})$. These regions each use different absorber materials: pure depleted Uranium for the EM, Uranium-niobium alloy (\%2) for the FH, and either copper (in the $\mathrm{CC}$ ) or stainless steel (EC) for the $\mathrm{CH}$. Each of these regions are further divided into multiple layers, each with its own unit cells. The EM calorimeter consists of the first four physical layers, the fine hadronic is three layers thick, and the last layer corresponds to the course hadronic calorimeter. 


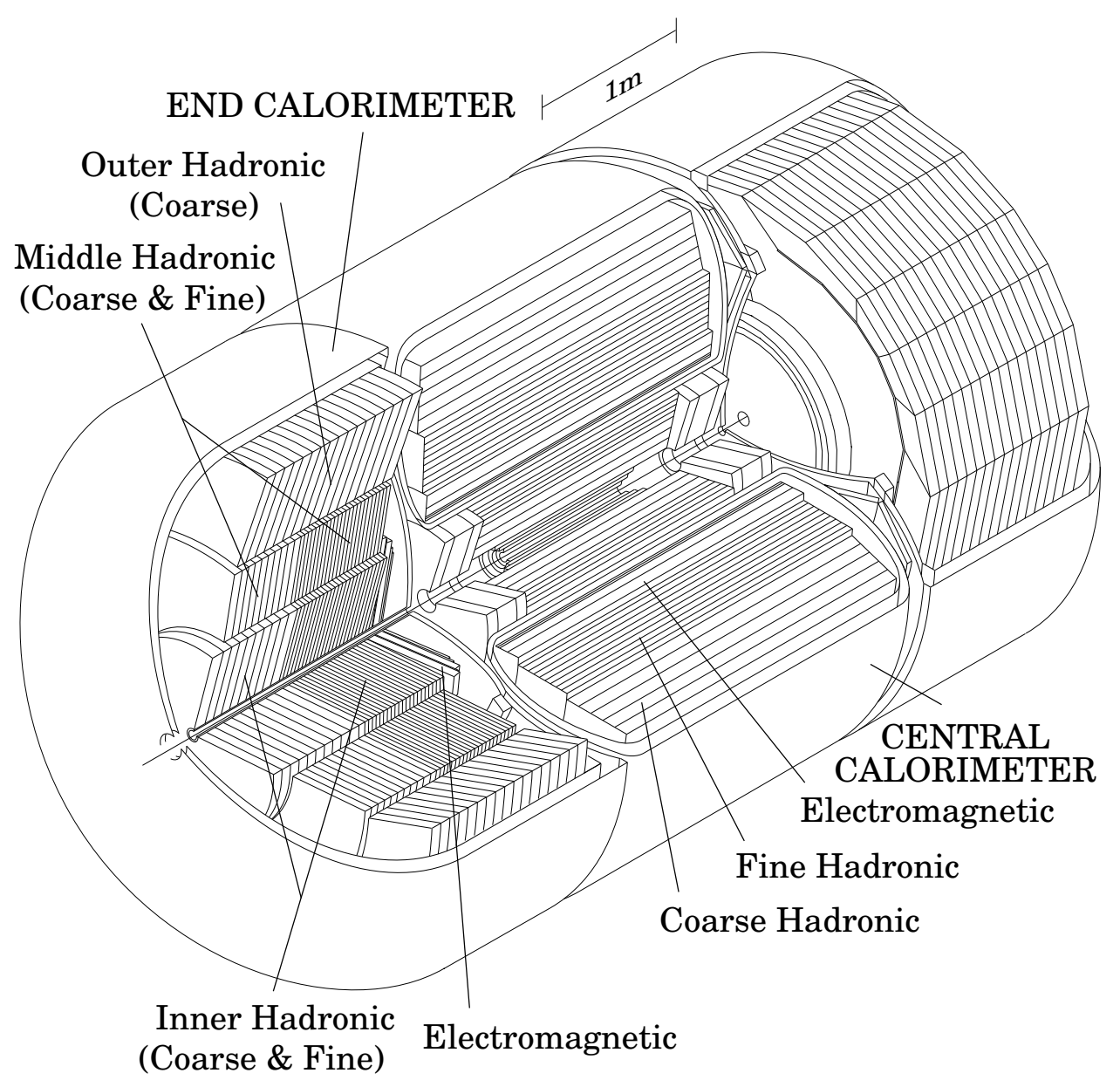

Figure 4.8: A 3-dimensional view of the D0 calorimeters. 


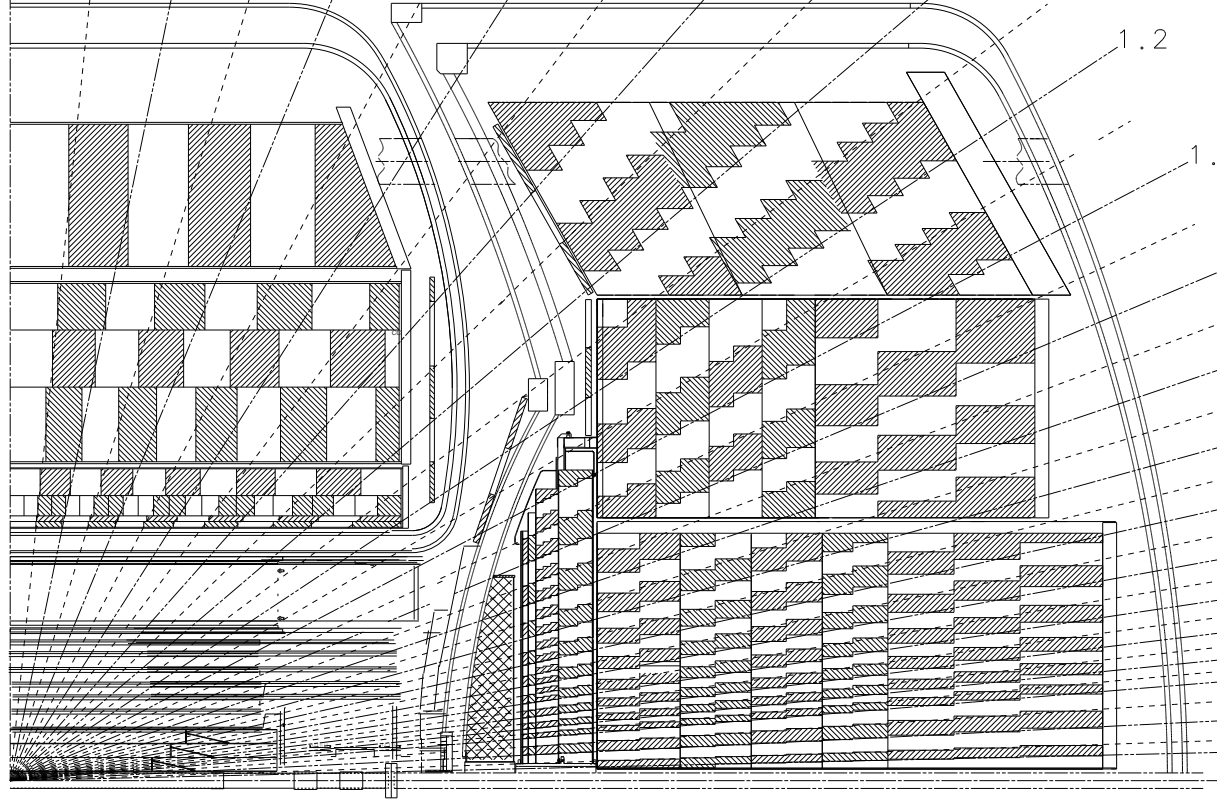

Figure 4.9: A side-view of one quarter of the detector, showing the projective tower structure in $\eta$ and depth. The lines extending from the center of detector correspond to constant pseudorapidities.

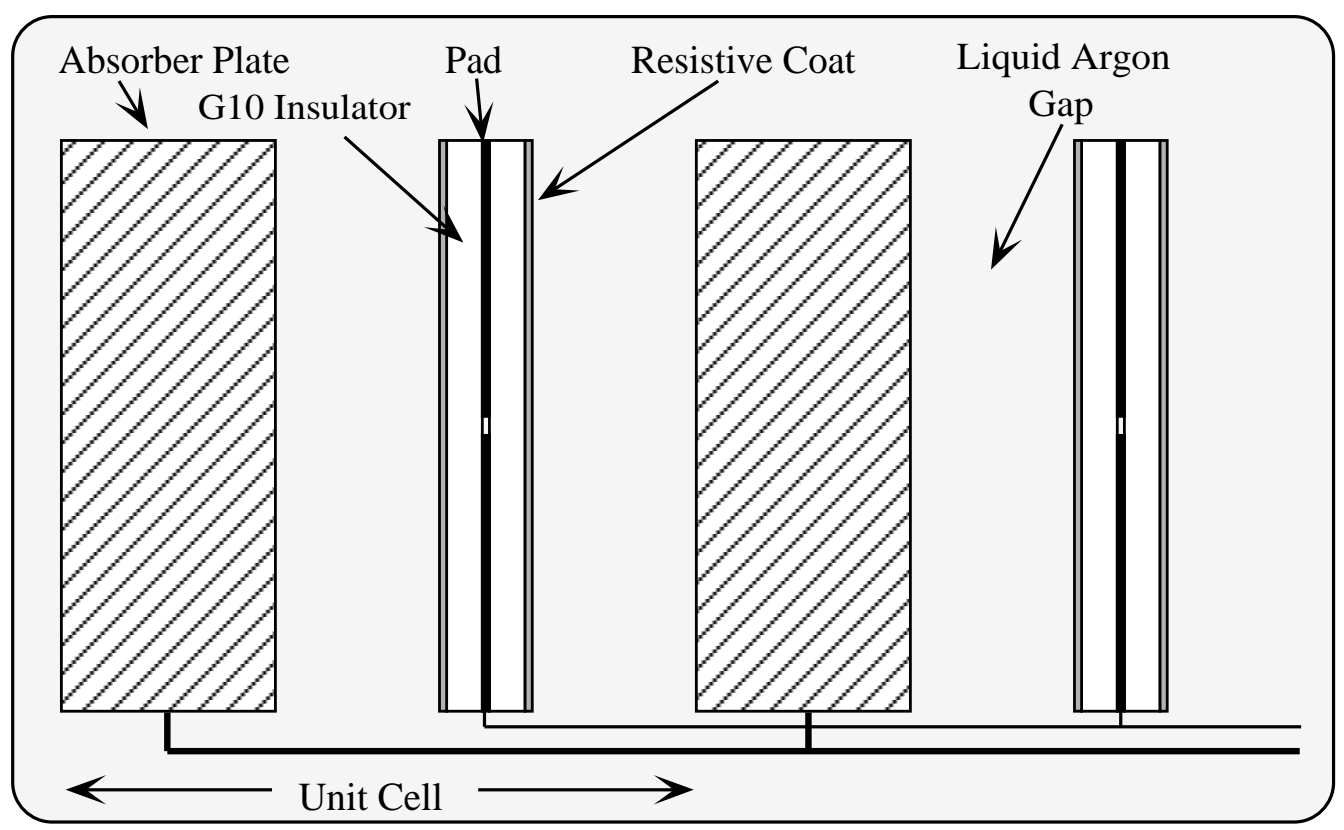

Figure 4.10: The schematic view of a typical unit cell, showing the gap structure, grounded absorber plates, and read-out boards. 


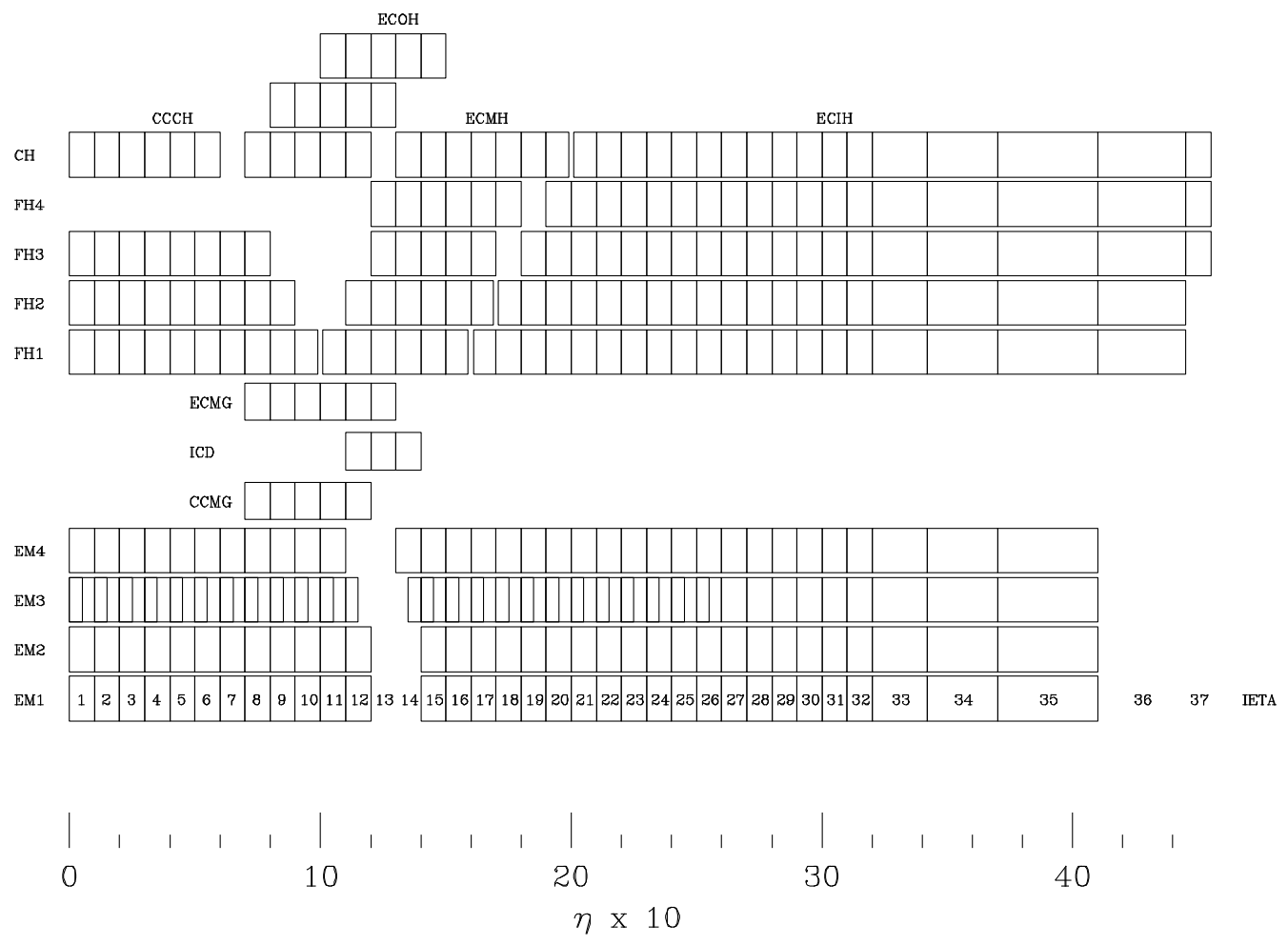

Figure 4.11: Map showing the configuration of calorimeter cells as a function of detector $\eta$ and depth. 
Each of these layers is further segmented into towers in $\eta$ and $\phi$. The precision towers divide the central calorimeter into 64 segments in the $\phi$ direction and 72 segments in $\eta$. The third physical layer [EM3] of the precision tower is further divided into four (2x2) cells for better shower shape information where the shower maximum was in Run I. The calorimeter is also divided into trigger towers, which are $2 \times 2$ arrays of precision towers, dividing the calorimeter in 32 segments in phi, and 37 in eta. Trigger towers are the smallest calorimeter units seen by the Level 1 Trigger. [40]

\subsubsection{Calorimeter Electronics}

The faster bunch crossing interval (132 ns) of Run II necessitated faster readout electronics [52]. Our precision measurements, nonetheless require low-noise and uniform performance from channel to channel.

The signal from calorimeter readout channels is triangular, with a very fast rise time and 400 ns decay time. This signal passes through a pre-amp which converts the charge to a voltage. The output signal approximates a step function with $400 \mathrm{~ns}$ rise time and $15 \mu$ s fall time. This signal is then sent through a filter/shaper that shortens the signal to a 320 ns rise time and 500 ns decay. This signal is sampled every $132 \mathrm{~ns}$ and the discrete sample signals are stored in switchedcapacitor arrays (SCA's), which serve as analog memory. Because residual signal from previous bunch crossings may remain in the electronics, the signal from the SCA's passes through baseline subtraction (BLS) boards, which measures the difference in voltage between the nominal signal and the last sample of the previous signal. The baseline-subtracted signal is stored in the level-2 SCA's, and on a positive trigger decision this signal is finally converted to a digital signal by ADC (analog-to-digital converter) cards [53].

\subsubsection{Muon System}

Muons produced with momenta higher than $1 \mathrm{GeV}$ at Tevatron collisions are minimum ionizing. These massive, high-energy particles with large mass-to-charge ratios deposit a roughly constant and minimal amount of energy when passing through materials. As a consequence, they are among the only particles to penetrate past the calorimeter system. The muon system is the 


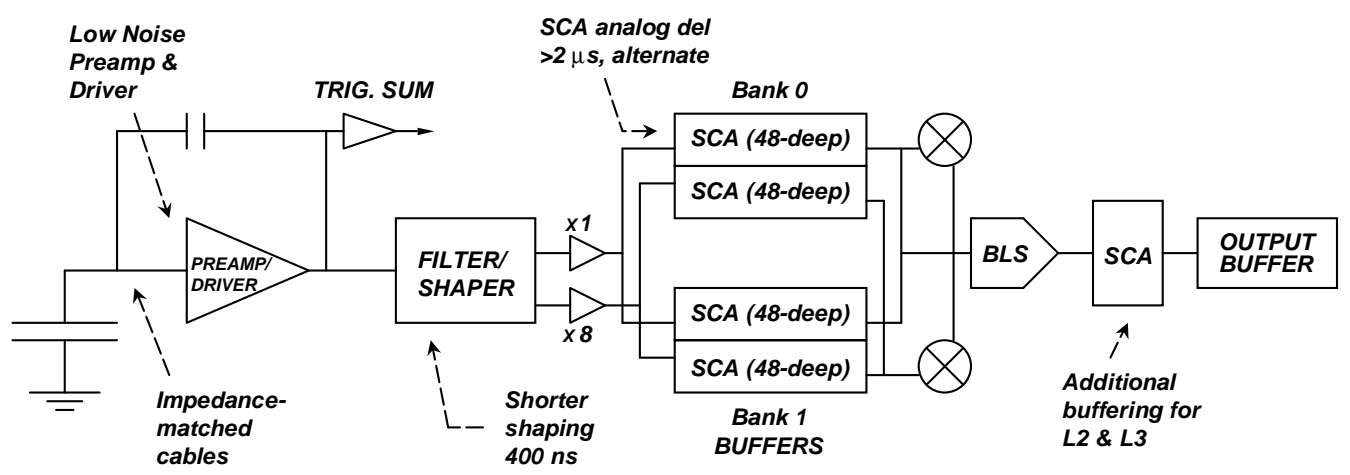

Figure 4.12: Schematic of the electronic read-out system.

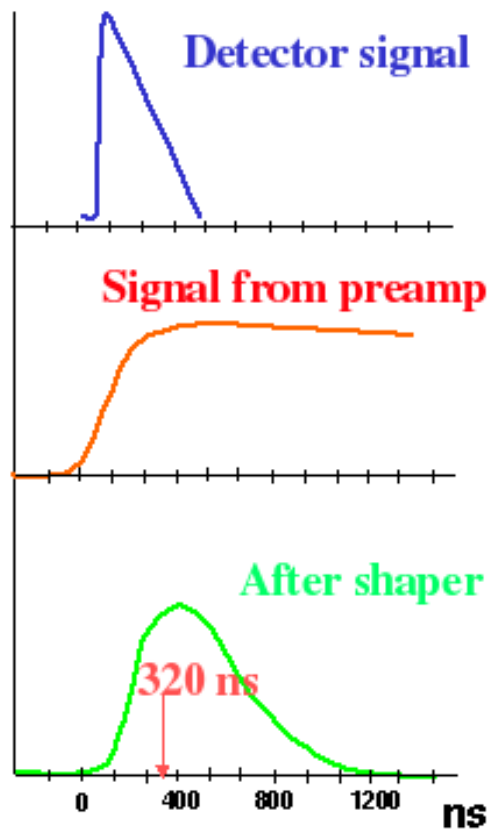

Figure 4.13: Typical signal shapes and various stages of the electronic readout. 
outermost subsystem of the D0 detector, designed to help identify muons and to provide additional tracking and momentum measurements.

The muon system consists of three layers, arranged with a rectangular geometry, with an iron toroid between the first and second layer. The toroid bends the muon trajectories and the deflection angle measured between the first and second layers can be used to determine the muon $p_{\mathrm{T}}$. The first two layers $(\mathrm{A}$ and $\mathrm{B})$ are proportional drift chambers. Drift chambers consist of vessels filled with gas and a wire anode. Charged particles ionize the gas, and the electrons drift towards the anode. The drift time is use to resolve position within the tube. The outermost layer (C) consists of scintillating pixels, used for triggering and position measurement.

Because this analysis uses the electron decay channel of $\mathrm{W}$ and $\mathrm{Z}$ events, we do not really use the muon system, although cosmic muons were used to study detector alignment.

\subsubsection{Luminosity Counters}

The luminosity monitors are used to detect inelastic $p \bar{p}$ collision. Positioned in the farforward region of the detector, this system consists of 24 plastic scintillating wedges at $\mathrm{z}= \pm$ $140 \mathrm{~cm}$ from the center of the D0 detector, radially surrounding the beam pipe and covering $2.7<|\eta|<4.4$. The luminosity monitor is used to measure the instantaneous luminosity in the detector. For the parameterized recoil model, the instantaneous luminosity is used as a parameter to describe the activity in the detector due to additional collisions, beyond the collision which produced the $\mathrm{W}$ or $\mathrm{Z}$ bosons. 


\section{Chapter 5}

\section{Optimizing the RunII Detector}

Upgrades to the $\mathrm{D} \varnothing$ detector in Run II have introduced a great deal more material in front of the calorimeter, along with new electronics, shorter integration times, and tighter zero-suppression cuts. Consequently, Run II detector performance differs greatly from Run I, when many of our analysis techniques were developed. The new inner tracking system with its $2 \mathrm{~T}$ solenoid and preshower detector, combined with material like the cryostat wall that was already present in Run I, total 4.0 radiation lengths of material before the first active layer of liquid argon [41]. In this chapter we discuss some of the calibrations and measurements undertaken to improve and better understand detector performance.

\subsection{Calibration}

The effects of physical and electronic non-uniformities in the calorimeter limit our ability to make precision measurements in Run II. Miscalibrations in the towers and cells of our finely segmented calorimeter have the effect of smearing the average energy resolution of the detector. This effect on our resolution corresponds to the "Constant Term" in traditional parameterizations of detector resolution, as will be discussed in Chapter 7 of this thesis. Since the effect of mechanical non-uniformities on the fractional resolution is energy independent, they are the dominant smearing

effect for high energy electrons. Moreover, extreme outliers among miscalibrated segments of the calorimeter introduce measurable, non-Gaussian effects that could also affect precision physics. The calibration process consists of two parts: calibration of the readout electronics, and calibration of the mechanical variations in the detector.

The electronics calibration consists of sending a pulse of known charge into the readout, and observing the measured charge. Since the pulser signal is inserted between the calorimeter and the electronics, reflections off of the calorimeter return to the readout and alter the shape and 
behavior of the pulse, thereby affecting the accuracy of the electronics calibration. Simulations of these reflections are still needed in order to fully understand and compensate for these differences. The technical details of the electronics calibration can be found in $[54,55]$.

The "gain calibration", which corrects for physical variations in the detector, involves adjusting the relative weights of the smallest possible calorimeter segments until their actual energy responses match their expected responses to within our desired precision. All of our calibration methods assume a proportional energy response in the calorimeter. Therefore, any non-linearities that remain after the electronics calibration cannot be corrected by the methods presented in this paper.

The best standard candle for absolute calibrations is the Z-peak, which is well known and provides relatively high statistics. In $1 \mathrm{fb}^{-1}$, one will typically find approximately 20,000 central Z-candidates after background subtraction [56]. Nonetheless, we still lack sufficient statistics to use the Z-peak alone for calibrating at the tower level or finer. We need a more inclusive sample to increase our statistical power. We proceed as follows:

1. We expect a uniform calorimeter response in $\phi$, since the beam physics of the Tevatron is not polarized. We look at generic collider events with EM energies above a threshold [57], and adjust the weights of calorimeter segments in $\phi$-rings of fixed $\eta$ to achieve a uniform relative response in that direction. This process is called "intercalibration" or "inter-phi calibration" [58, 59, 60]. Enough data was collected to intercalibrate the CC region to within one percent on a precision tower basis and to within $2 \%$ on a layer level.

2. Once we have eliminated the $\phi$ degree of freedom, we have enough $Z \rightarrow e e$ events to absolutely calibrate each intercalibrated $\eta$ ring.

Using this procedure we have succeeded in providing calibration constants on the precisiontower level in the EC region and on a per-layer basis in the CC region. These calibration constants, along with an independent electronics calibrations have been implemented into the p17 release series of the D0 offline reconstruction program. More details on the calibrations used in this analysis can be found in [61]. 


\subsubsection{Intercalibration In $\phi$ Using Generic Collider Data}

At the precision tower level, we equalize the occupancy of events above an energy cut $L$ for each tower. Monte Carlo studies have shown that little statistical power is gained by using shape information. Each tower $i$ in the $\phi$-ring has its transverse energies $E_{i}$ corrected by a multiplicative constant:

$$
E_{\mathrm{T}}^{i \prime}=\alpha_{i} E_{\mathrm{T}}^{i}
$$

The parameters, $\alpha_{i}$ are adjusted so that the number of events with the energy $E_{i}^{\prime}$ above $L_{i}$ for each tower is equal to the average occupancy over all towers in the ring.

We also calibrate each of the four physical layers [EM1,EM2,EM3,and EM4] within each precision tower. The basis for our physical layer calibration is a fit each layer's unique fraction of the total tower energy. The first of the four layers is kept at a fixed calibration of unity, and the relative calibrations, $\beta_{i}^{2}, \beta_{i}^{3}$, and $\beta_{i}^{4}$ of the other three layers are adjusted so that the distribution of fractional energy in a particular layer of a particular tower matches the shape of the average distribution for that layer over all $\phi$.

Figures 5.1 and 5.2 show an example of the energy fraction distributions for the layers of a tower compared to the averaged reference distributions, before and after calibration.

Minimization is performed by the SIMPLEX algorithm of MINUIT [36], an iterative search method that is available in the CERN program library.

The challenge with combining the tower and layer intercalibrations is that the tower calibration can move events in and out of the offline cuts, changing the final value of the layer calibrations and vice versa. As a result, one iteration of the intercalibration process does not produce stable, self-consistent constants. Rather, only after many iterations of the calibration procedure do the fluctuations from the two independent calibrations (layer and tower level) dampen and converge to fixed values. Experience has shown that 30 iterations of the calibration procedure is a safe number to insure convergence to stable set of constants. 

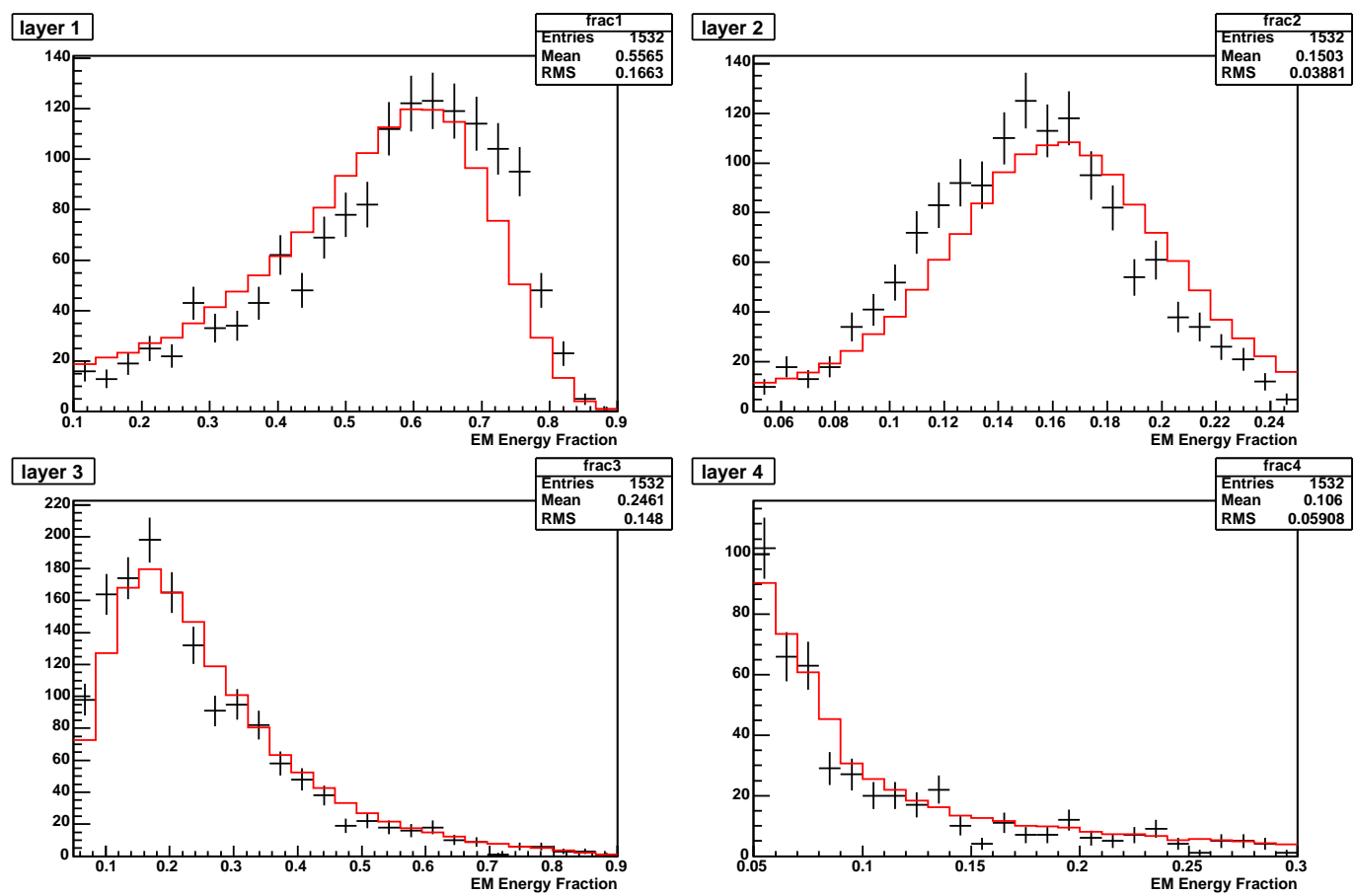

Figure 5.1: Comparison between the four EM fraction distributions of an uncalibrated tower (points) and the average distributions for the eta ring (solid)

\subsubsection{Absolute Calibration of Rings in $\eta$ Using $Z \rightarrow e e$ data}

Absolute calibration of the rings in $\eta$ is performed using a $Z \rightarrow e e$ sample. Multiplicative calibration constants $c_{i \eta(j)}$ on the raw energies for electrons that fall in the given $\phi$-ring of fixed $i \eta=j$. We apply these constants to the raw electron energies in each $i \eta$, and compute the invariant $Z$ boson mass, given by

$$
m_{Z}^{i}=E_{\text {elec } 1}^{i} E_{\text {elec } 2}^{i}(1-\cos \delta \theta)
$$

where $E_{\text {elec } j}^{i}$ is the reconstructed electron energy for the $j$ th electron in the $i$ th event after applying various material-based and geometric based energy-loss corrections to the raw-energies, which are given by

$$
E_{\text {elec } j}^{i}=E_{\text {elec } j}^{i}\left(c_{i \eta(j)} \operatorname{Eraw}_{\text {elec } j}^{i}, \alpha\right)
$$



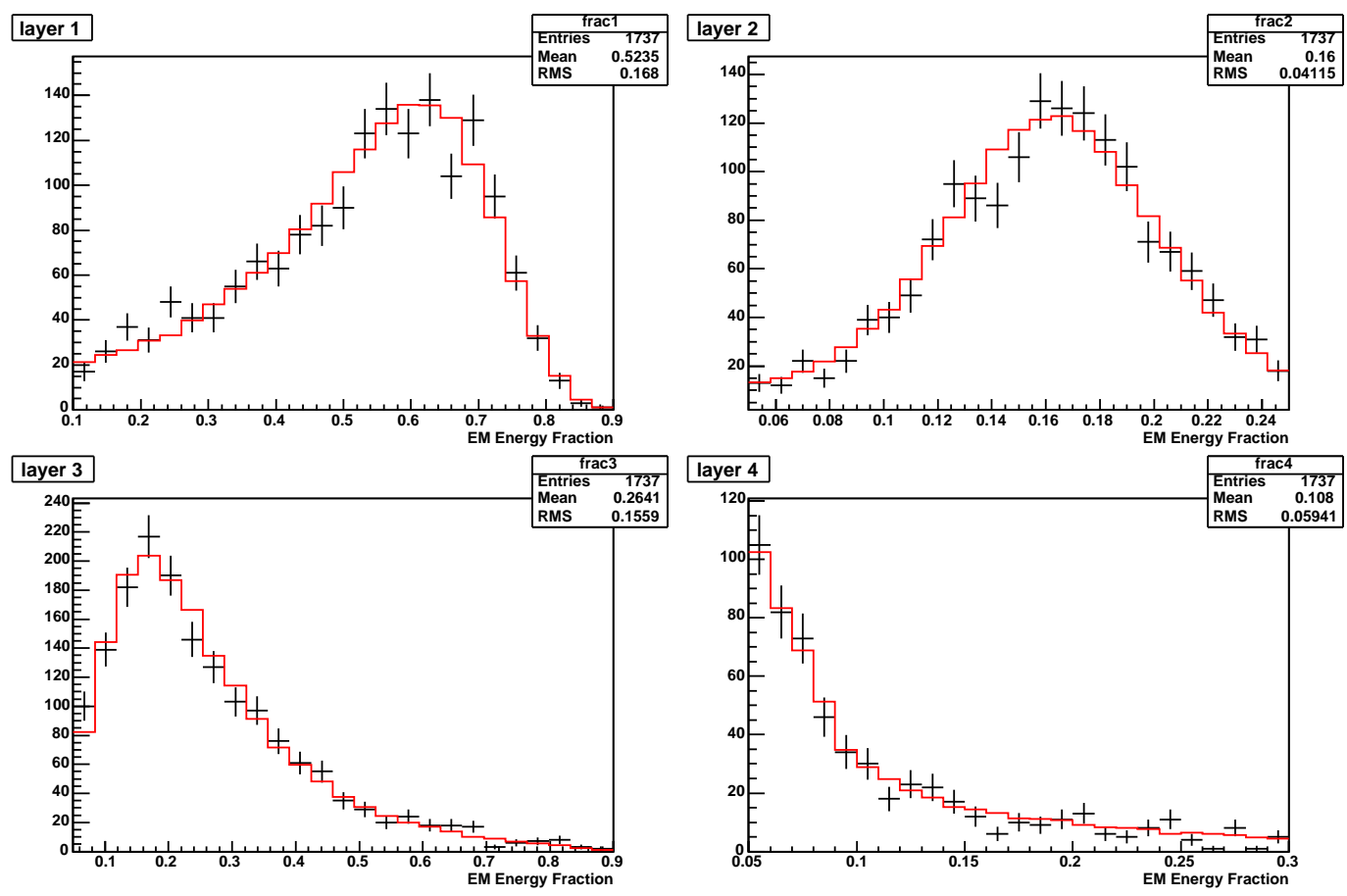

Figure 5.2: Comparison between the four EM fraction distributions of an uncalibrated tower (points) and the average distributions for the eta ring (solid), after calibration

and $\alpha$ is the array of parameters for those energy loss corrections.

The invariant mass distribution of these $m_{i}$ for all events $i$, should approximate a voigtian distribution with central value equal to the experimentally known $Z$ boson mass $M_{Z}$ and width determined from full $\mathrm{MC}$ expectations of the detector resolution, combined with the appropriate backgrounds.

We adjust these calibration constants $c_{i \eta(j)}$ and recompute the invariant mass distribution until the central value approaches the LEP $Z$ boson mass value and the width of the distribution is minimized to the full MC predicted resolution.

We do this for the first $26 i \eta$ values, and $c_{27}$ corresponding to $27 \geq|i \eta| \leq 37$. 


\subsubsection{Result of Calibration}

Resulting temperature plots of the layer level calibration are shown in Fig 5.4. The effects of physical non-uniformities can be seen. For example, the red band of tower calibration constants for $\phi=34,35$ in the first two layers, corresponds to a known deformity that occurred in the assembly of $\phi$-module 17 of the calorimeter.

The final effect of applying these calibration constants was a $10 \%$ improvement in the resolution of the $Z$ boson invariant mass distribution, which is now close to the $2 \%$ precision the detector was designed for. The $Z$ boson invariant mass distributions before and after the calibration are shown in Fig 5.5.

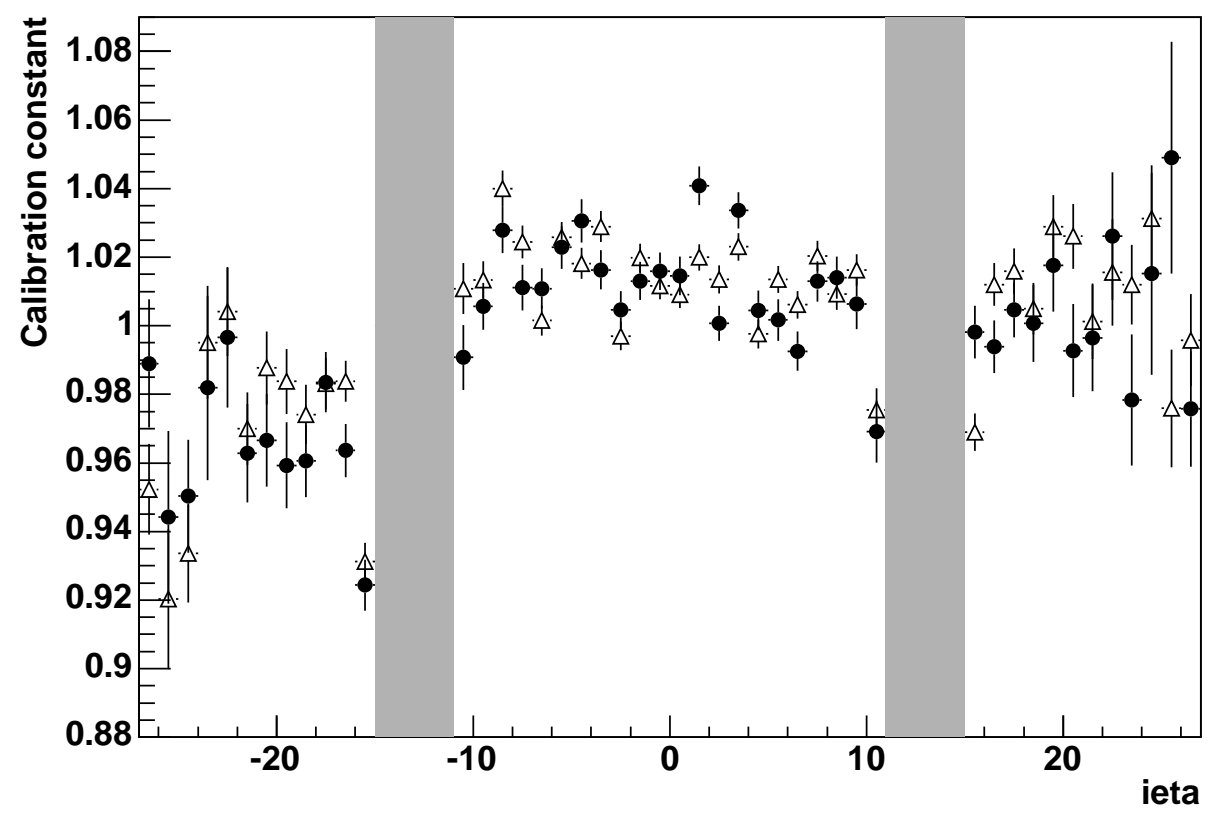

Figure 5.3: Result of the calibration fit to data. The horizontal axis represents ieta and the vertical axis represents the fit result for $c_{\text {ieta }(j)}$. The grey areas show regions in which we do not attempt to determine $c_{\text {ieta }(j)}$. The point at ieta $=-27$ represents the combined $c_{\text {ieta }(j)}$ for $-37 \leq$ ieta $\leq-27$, and the point at ieta $=27$ represents the combined $c_{\text {ieta }(j)}$ for $27 \leq$ ieta $\leq 37$ (see text). The triangles represent the result for data taken before the Sept-Nov 2003 shutdown, and the dots represent the result for data taken after that shutdown. 

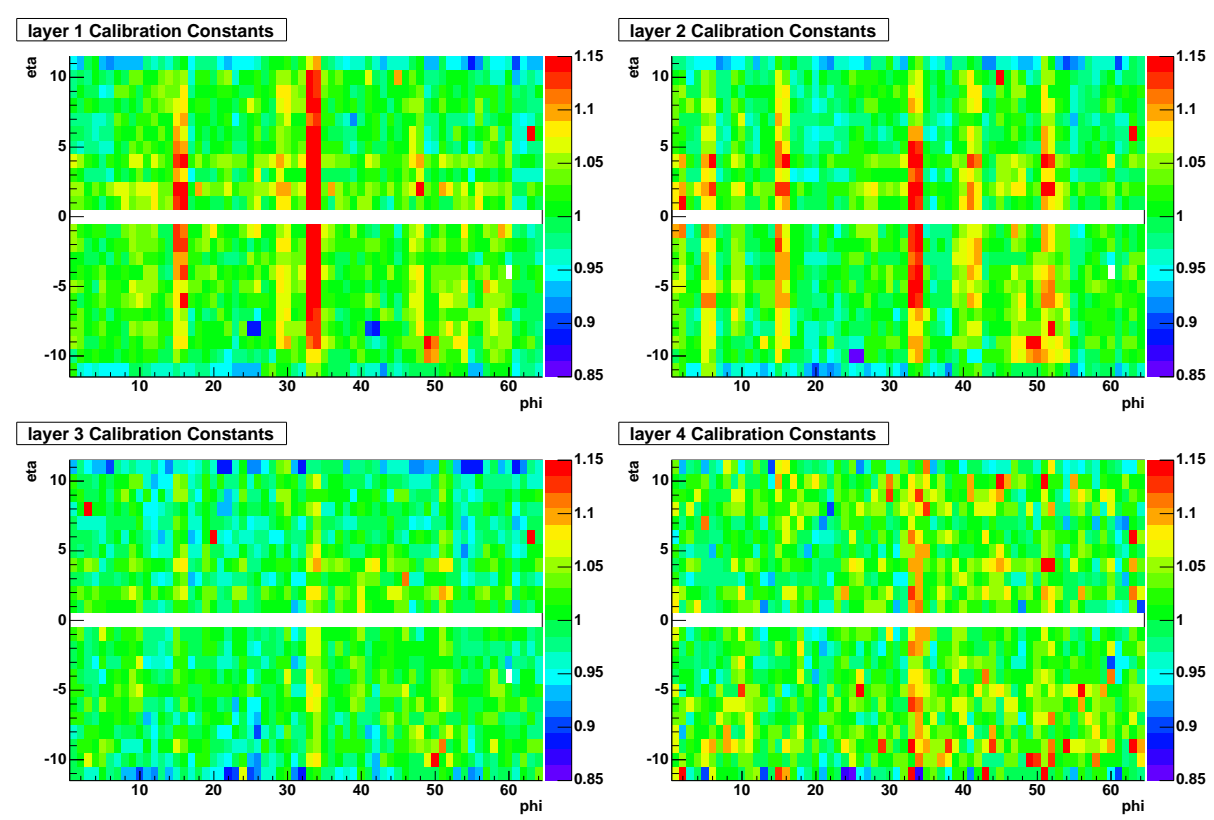

layer 4 Calibration Constants

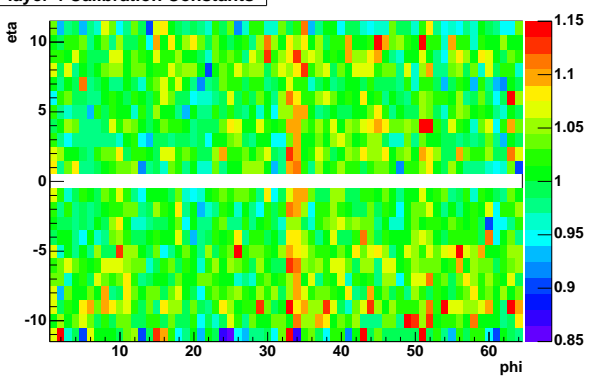

Figure 5.4: Temperature plots of the inter-calibration constants for EM1 (upper left), EM2 (upper right), EM3 (lower left), and EM4 (lower right). 

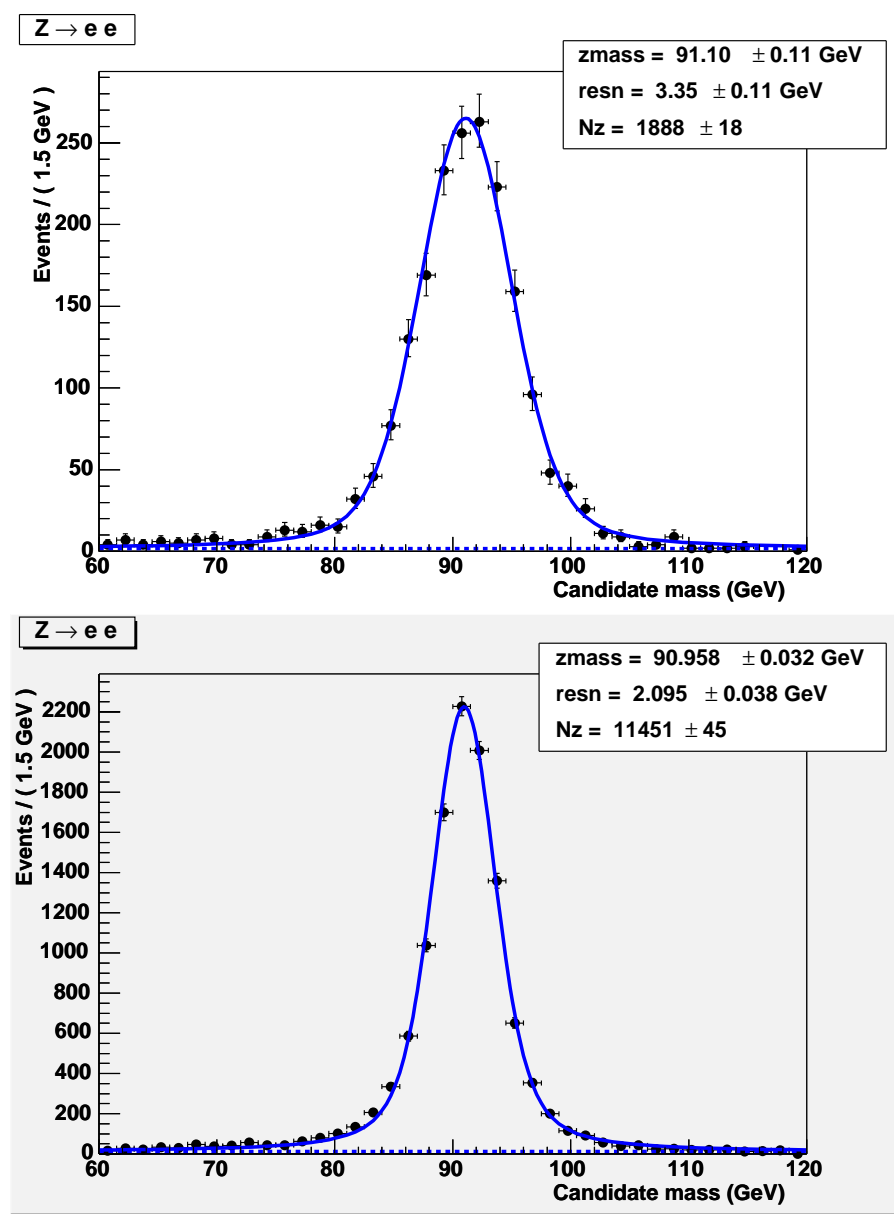

Figure 5.5: The Width of the Z-peak for two CC elections, before (top) and after (bottom) the in-situ calibration was performed. Resolution improves by roughly $10 \%$. 


\subsection{Energy Loss Corrections}

With so much material in front of the calorimeter in Run II, the actual energy response of the detector is very non-linear. For example, one would not expect the energy of a $5 \mathrm{GeV}$ electron passing through the roughly $4 X_{0}$ of dead material before the first active layer to be proportional to the energy response of a $30 \mathrm{GeV}$ electron. GEANT-based energy loss corrections based on our best material description of the calorimeter are applied to our reconstructed electron energies to linearized the energy response.

\subsection{Material Studies}

Studies using GFLASH [63], a parameterized shower simulation suggest that new material has changed the nature of the calorimeter's EM shower development and, with it, the kinematic dependences of the resolution. Figure 5.6 shows the average shower development as a function of depth produced using a simple parameterized shower simulation. Two major features stand out. First, much of the energy at the beginning of the shower is lost in dead material. Second, the shower maximum is no longer entirely contained in EM3 as it was in Run I. Figure 5.7 shows the longitudinal shower development of 10 individual showers at two different pseudorapidities. Not only does the longitudinal profile fluctuate event-by-event, but the nature of those fluctuations is different for the two angles of incidence. This implies a strong angular dependence of the sampling resolution not observed in the Run I. Figure 5.8 shows 10 fluctuations as measured at two different fixed energies. These fluctuations show a strong dependence on energy, as well.

Using Run II GEant Monte Carlo, we have re-examined the energy and $\eta$ dependence of the resolution. Figure 5.9 shows the $\eta$ dependence of the energy resolution for single electrons in GEANT, fit with a curve and compared with the Run I dependence. Figure 5.10 shows the equivlent plots of the energy resolution due to sampling fluctuation as a function of energy for single CC electrons in GEANT. The results of these detector simulations are markedly different from those observed Run I. These new dependences have motivated the development of a new parameterized model for the energy resolution of electromagnetic particles, described further in Chapter 7. 
The new parameterization contains too many degrees of freedom to tune to the limited set of available data observables alone. Ideally, the model can be tuned to the GEANT-based, full detector simulation. However, the standard GEANT-based model must first be checked against data. Observed disagreements between the data and full Monte Carlo suggest that there is material missing from the full detector model [64]. Thus, in order to build an accurate parametric description of the electron energy resolution, the amount of material missing from the full MC must first be determined. The missing material can then be included in a special, corrected full MC model that is used to tune the Run II parameterization.

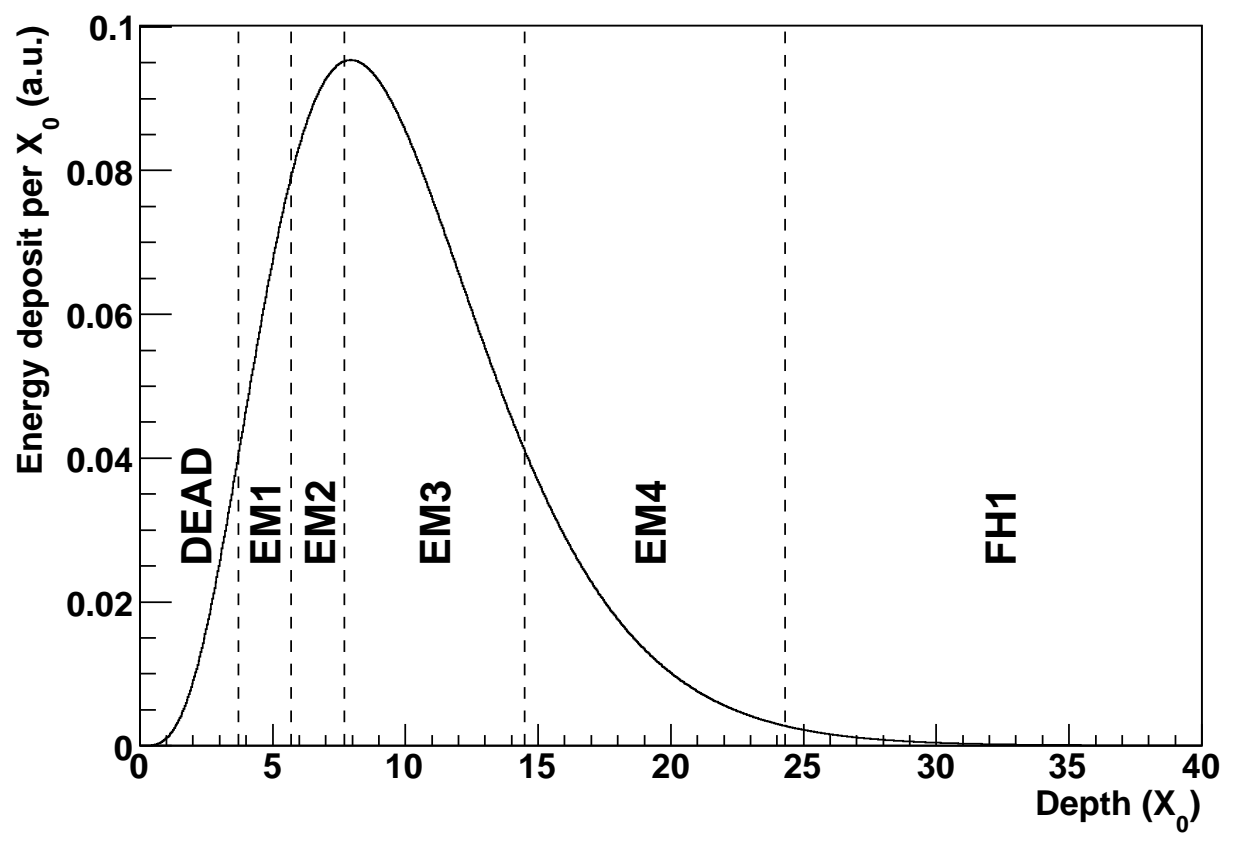

Figure 5.6: The average energy deposited at a function of depth (in radiation lengths) for $45 \mathrm{GeV}$ single-electrons at normal incidence, as estimated using a parameterized shower simulation.

Two data samples are examined in depth: $Z \rightarrow e e$ decays and $J / \psi \rightarrow e e$. Both of these samples contain electrons that explore the full angular dependence of the resolution, and decay electrons from the two resonances have very different $p_{\mathrm{T}}$ spectra, helping to constrain the energy dependence of the resolution. The $J / \psi \rightarrow$ ee resonance, is at low enough energies that the resolution is dominated by material effects on the energy sampled. Longitudinal shower profiles in 

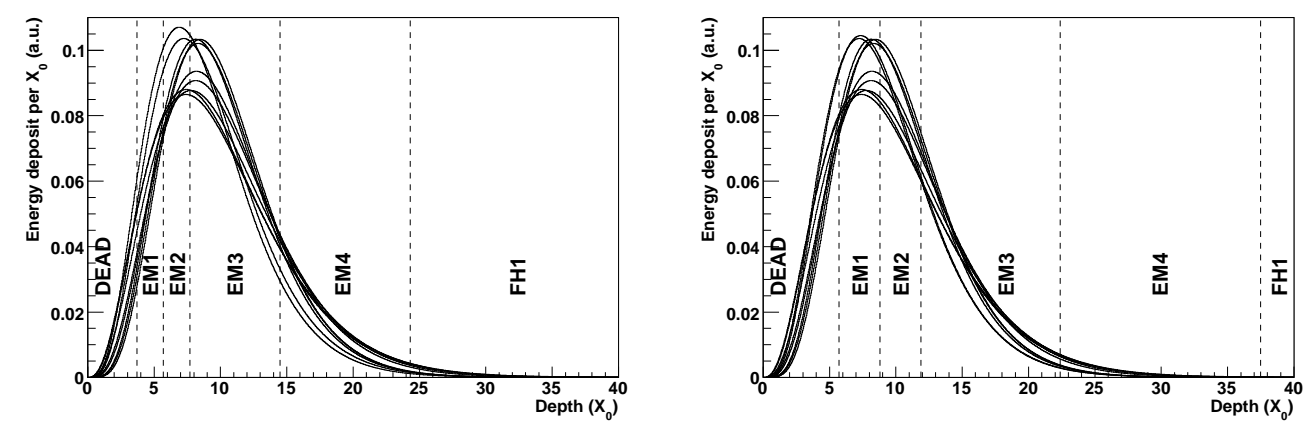

Figure 5.7: The energy deposited at a function of depth (in radiation lengths) for $45 \mathrm{GeV}$ singleelectrons at normal incidence (left) and $\eta=1.0$ (right) as estimated using a parameterized shower simulation. Each line represents one particular shower drawn from a parameterized model for shower fluctuations [63]. One clearly sees the shift of the shower earlier in the calorimeter as the angle of incidence increases.

$Z \rightarrow e e$ data are particularly sensitive to material in the detector.

Both samples contain two electrons, each with its own pseudo-rapidity, so we cannot measure resolution directly as a function of angle. Rather, we divide our data into kinematic categories corresponding to the various permutations of $\left|\eta_{\text {phys }}\right|$ of both electrons. We start by dividing $|\eta|$ into five bins. Table 5.1 shows the eta ranges corresponding to each bin. Then, the 15 categories correspond to all of the possible, unique combinations of the $\eta$ bins in which the two electrons can fall. The numbering convention for these categories is described in Table 5.2. For example, category 11 represents one electron in bin 1 and one electron in bin 2. Similarly, category 12 refers to one electron in bin 1 and one electron in bin 3 .

We produce template distributions of the measured $J / \psi$ resonance, at varying sampling resolutions, and use these templates to fit for the sampling resolution as a function of the kinematic categories in $\eta_{\text {phys }}$. Template production starts with generated $J / \psi \rightarrow e e$ events and generated events corresponding to the nearby $\psi(2 s)$ resonance. These two resonances, along with a generic background are combined in the proper proportions, as measured using data with tracking information. Because the low $p_{\mathrm{T}}$ decay electrons from the $J / \psi$ sit close to our trigger turn-ons at D $\varnothing$, 


\begin{tabular}{l|l} 
Eta Bin & Physics Eta Range \\
\hline 0 & 0. to 0.2 \\
1 & .0 .2 to 0.4 \\
2 & 0.4 to 0.6 \\
3 & 0.6 to 0.8 \\
4 & more than 0.8
\end{tabular}

Table 5.1: The five bins into which we divide the $\left|\eta_{\text {phys }}\right|$ of central electrons

\begin{tabular}{|c|c|c|}
\hline Category & Electron 1 Bin & Electron 2 Bin \\
\hline 10 & 0 & 0 \\
\hline 11 & 0 & 1 \\
\hline 12 & 0 & 2 \\
\hline 13 & 0 & 3 \\
\hline 14 & 0 & 4 \\
\hline 15 & 1 & 1 \\
\hline 16 & 1 & 2 \\
\hline 17 & 1 & 3 \\
\hline 18 & 1 & 4 \\
\hline 19 & 2 & 2 \\
\hline 20 & 2 & 3 \\
\hline 21 & 2 & 4 \\
\hline 22 & 3 & 3 \\
\hline 23 & 3 & 4 \\
\hline 24 & 4 & 4 \\
\hline
\end{tabular}

Table 5.2: Definitions of the kinematic categories in $\left|\eta_{\text {phys }}\right|$ that we use to label CC-CC dielectron events 

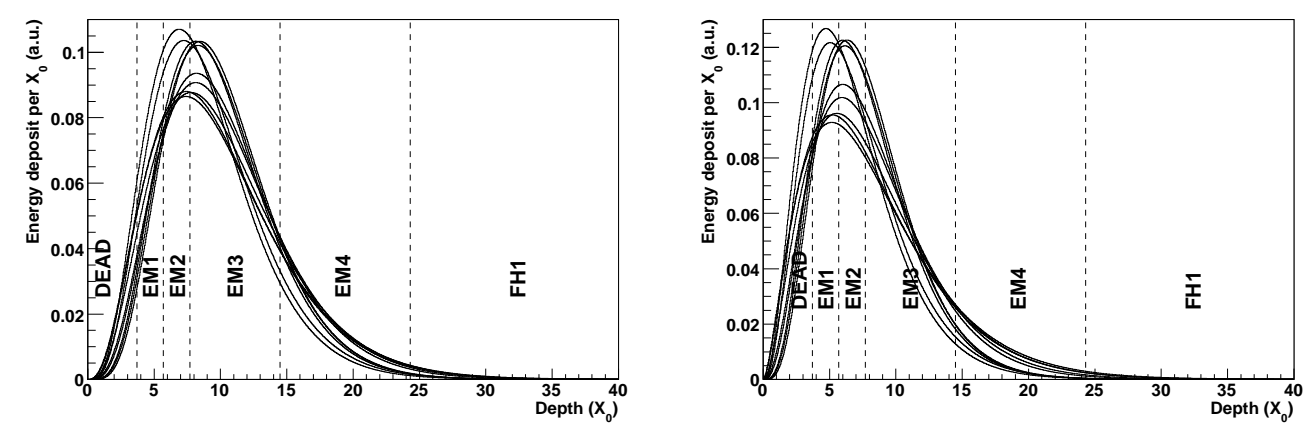

Figure 5.8: The energy deposited at a function of depth (in radiation lengths) for $45 \mathrm{GeV}$ (left) and $5 \mathrm{GeV}$ (right) single-electrons at normal incidence. Each line represents one particular shower drawn from a parameterized model for shower fluctuations [63]. One clearly sees the large shift of the shower earlier in the calorimeter at lower energies.

a sophisticated trigger-efficiency model is also necessary. Finally we apply a simple detector model to produce $J / \psi \rightarrow e e$ at various values of the sampling resolution. Since energy scale and offset were not applied, we also kept a floating energy offset and fit our data with a 2-parameter fit to templates that vary in both resolution and energy offset, although we ignore the offset value.

Figure 5.11 shows the fit to a $J / \psi \rightarrow e e$ sample over all kinematic categories, using our model. Although the shape is complicated and non-gaussian, we are able to accurately reproduce its key features. We measure the sampling resolution versus kinematic categories in $\eta$ in two different data-sets, corresponding to different trigger definitions. We ultimately use the results from the measurement of what we call the "trigger-low" data set, and use the "trigger high" set as a cross-check. Figure 5.12 shows the results for the "trigger low" set, with overall and statistical error bars. A more in-depth treatment of this measurement can be found in [66]. The average sampling resolution over all categories is much larger than predicted by GEANT (shown by the dashed-line), suggesting that indeed there is more material in the real detector.

We fit for missing material in the GEANT model by simultaneously comparing the fractional energy in each of EM layers of the detector for all bins in $\left|i \eta_{\text {det }}\right|$. We adjust the amount of unknown material until the longitudinal shower develop versus $\eta$ of the full MC matches that of the data. 


\section{Fractional energy resolution at $\mathrm{E}=\mathbf{4 5} \mathrm{GeV}$}

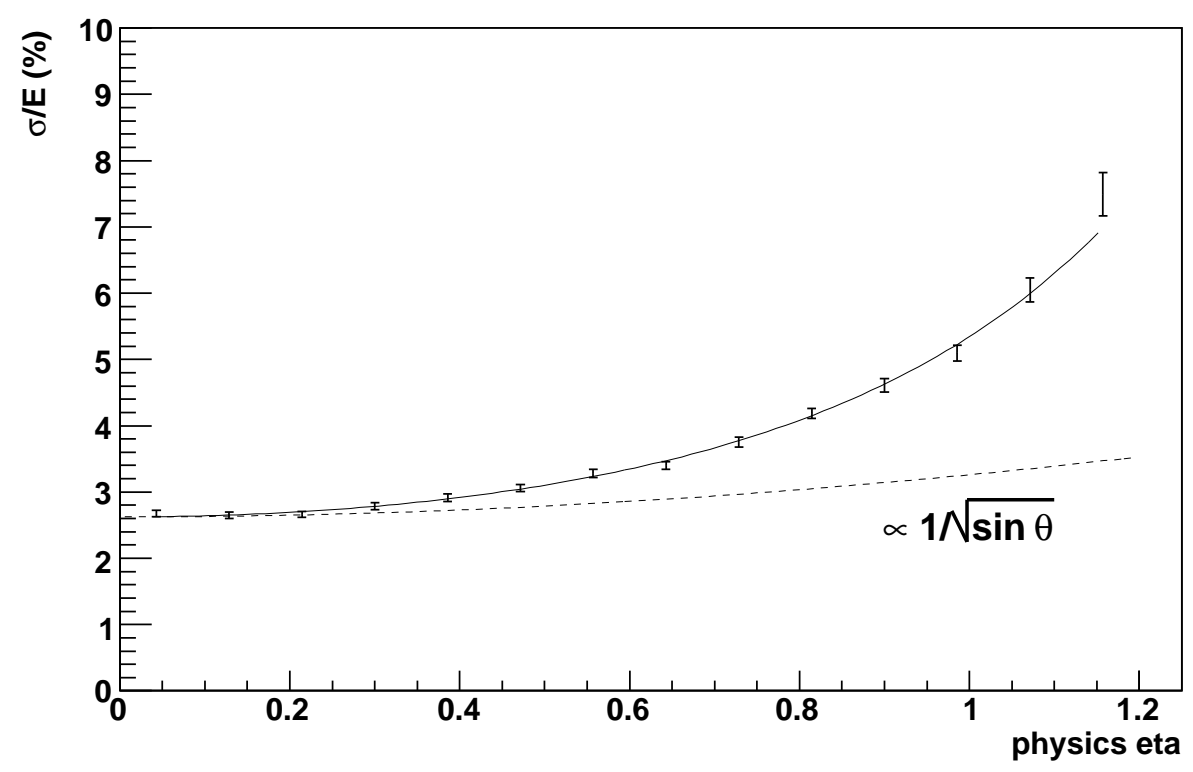

Figure 5.9: Fractional Energy Resolution for $45 \mathrm{GeV}$ electrons as a function of physics $\eta$, in GEANT Monte Carlo. The data points are generated from full GEANT Monte Carlo of single electrons. The solid curve is a fit using the parameterization described in Chapter 7 and the dashed line shows a $1 / \sqrt{\sin \theta}$ dependence.

We hypothesize that the missing material comes from a slight under-estimate of the thickness of cylindrically uniform bulk material in the detector, likely the solenoid coil, which is difficult to model. Copper is chosen as the material to vary. Comparisons using our $J / \psi$ and $Z$ measurements, after the missing material is inserted, show that this assumption gives very good data agreement for many different kinematic categories and two different data samples.

Figure 5.13 shows the fit result for missing material. Figure 5.14 shows the fit result for missing material in each layer of the calorimeter independently. Event fitting based on the fractional energy distribution in each individual layer gives consistent results. We find that GEANT is missing an amount of material equivalent to a uniform, cylindrical distribution of copper with a thickness in radiation lengths of $n X_{0}=0.1633 \pm 0.0095$.

Figures 5.15 and 5.16 show MC-data comparisons of the longitudinal shower profile for 


\section{Fractional energy resolution at normal incidence}

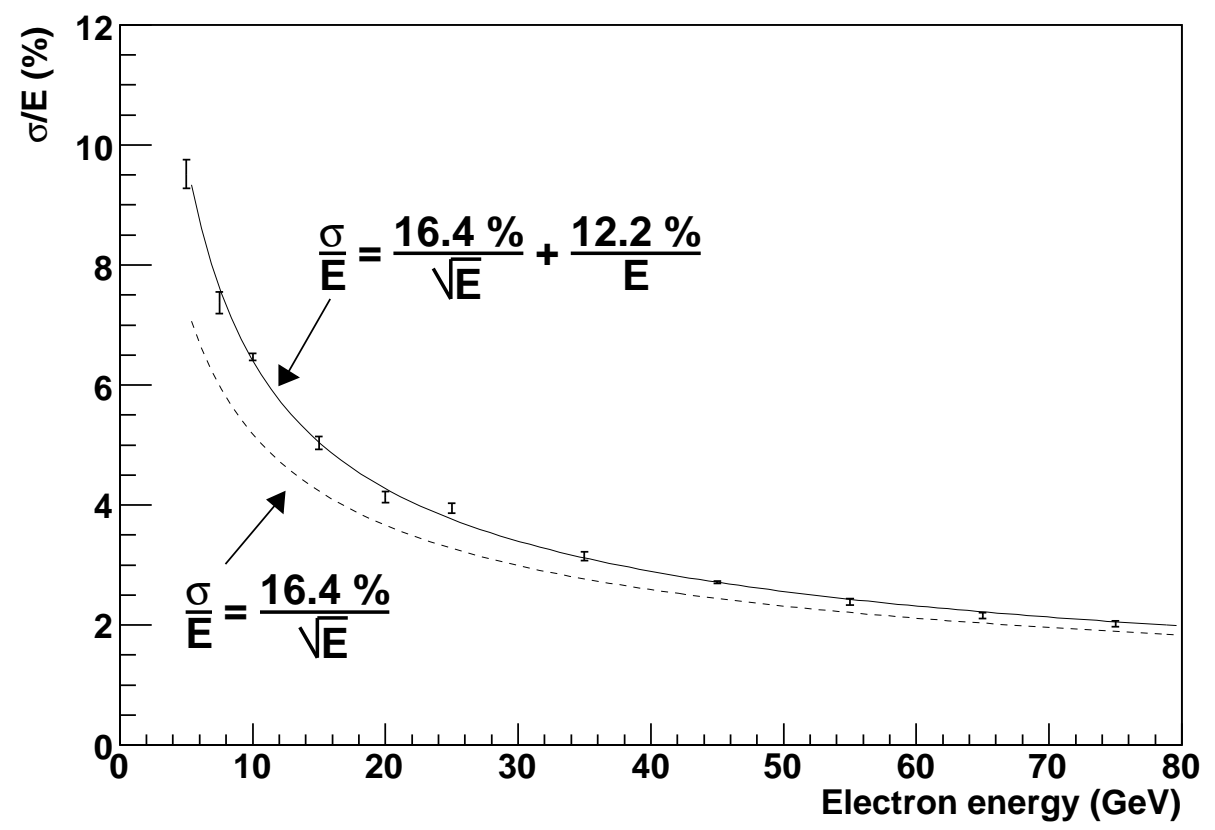

Figure 5.10: The fractional energy resolution due to sampling effects for electrons at normal incidence as a function of energy. The data points are generated from full GEANT Monte Carlo of single electrons. The dashed line represents a $1 / \sqrt{E}$ dependence and the solid line is a fit using the new parameterization described in Chapter 7 .

category-23 $Z$ electrons before and after the missing material is added back in. The missing material simultaneously corrects the fractional energy distributions in each EM layer of the calorimeter. 


\section{JPsi Resonance for LOW Triggers (Entire CC)}

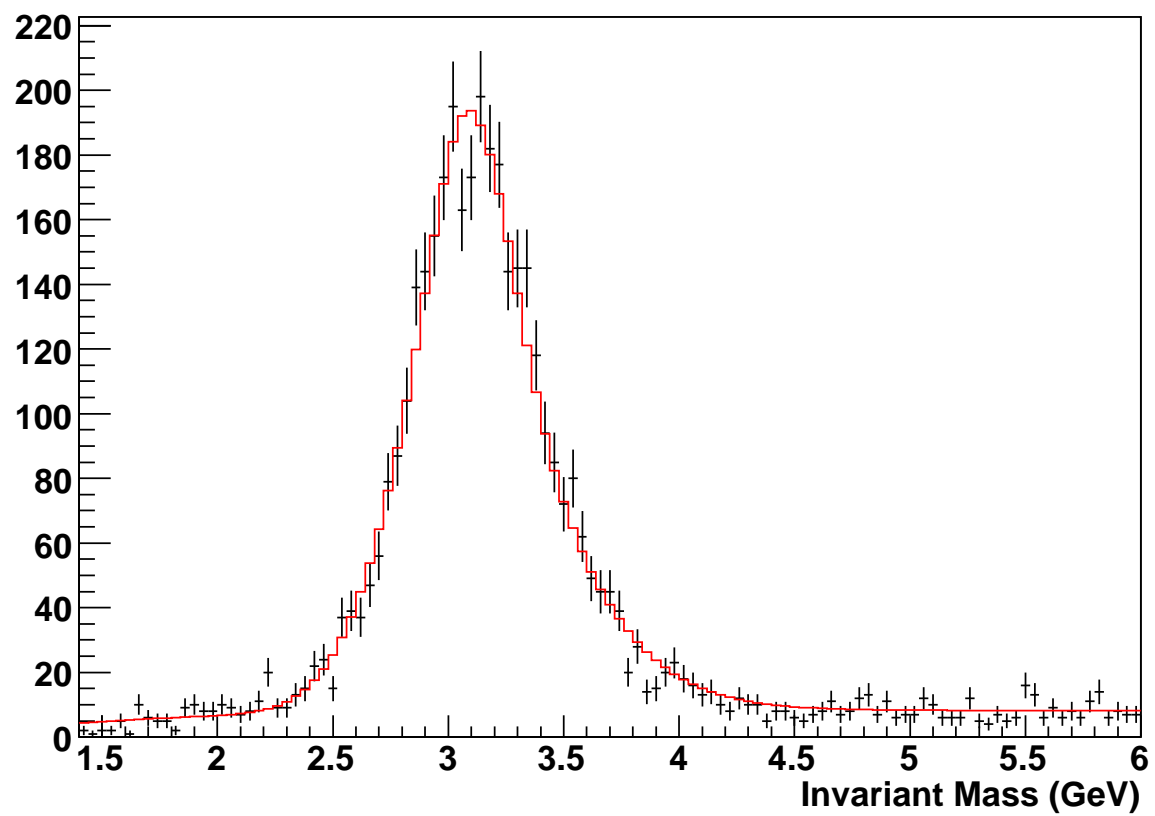

Figure 5.11: Fit result to the $J / \psi$ resonance in "trigger low" data. 


\section{Sampling Term vs Kinematic Category}

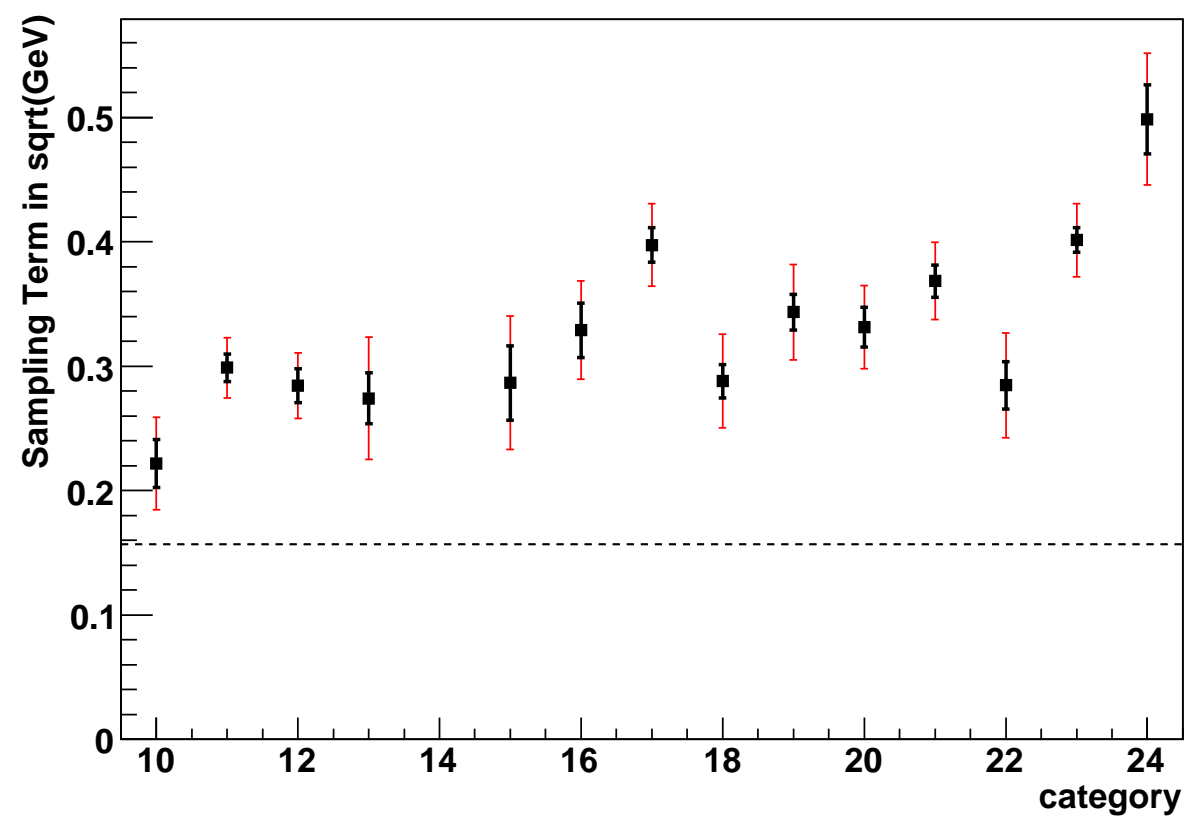

Figure 5.12: Sampling resolution measured at the $J / \psi$ versus kinematic category. The dark error bars indicate the systematic uncertainty and the light error bars are the total uncertainty. The dashed line indicates where the GEANT derived sampling resolution is. The discrepancy points to missing material in the full simulation. 


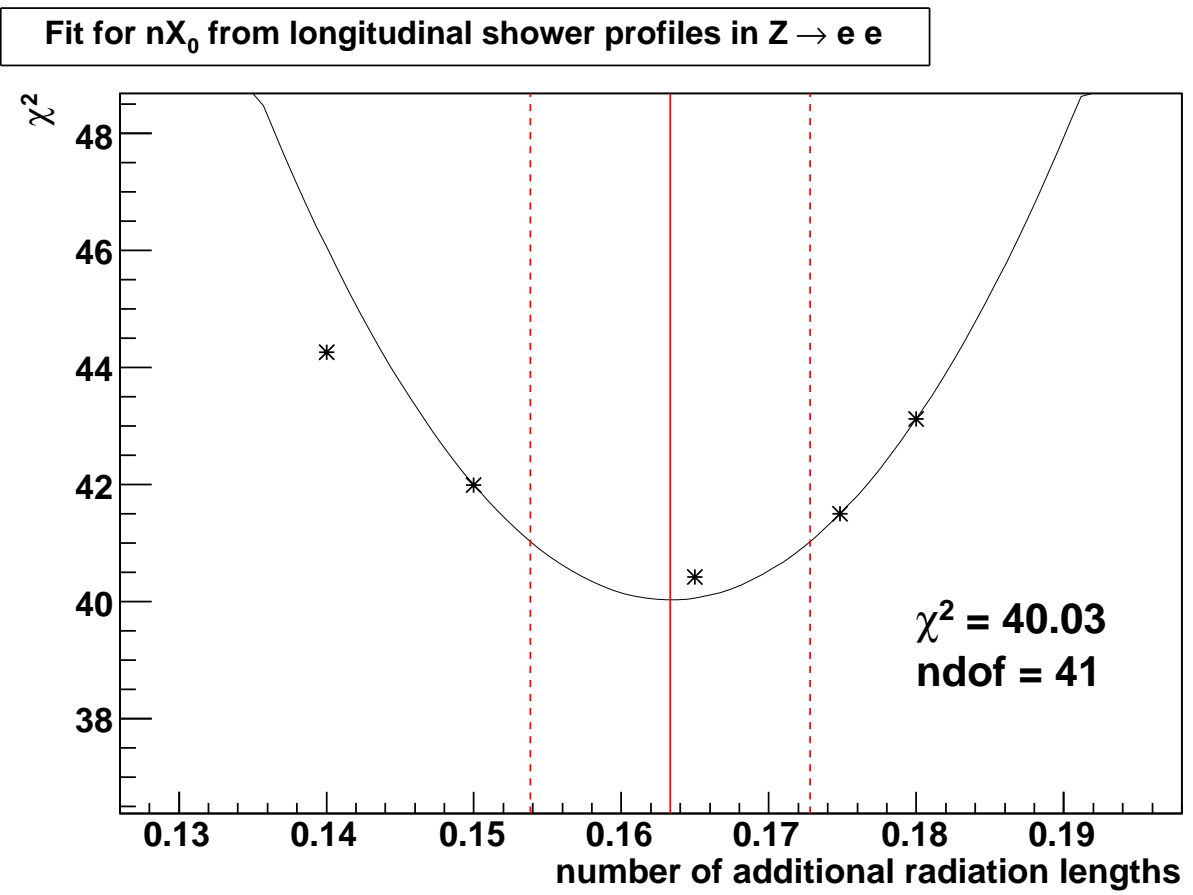

Figure 5.13: Likelihood function for the final fit results for missing $X_{0}$ in the full detector simulation.

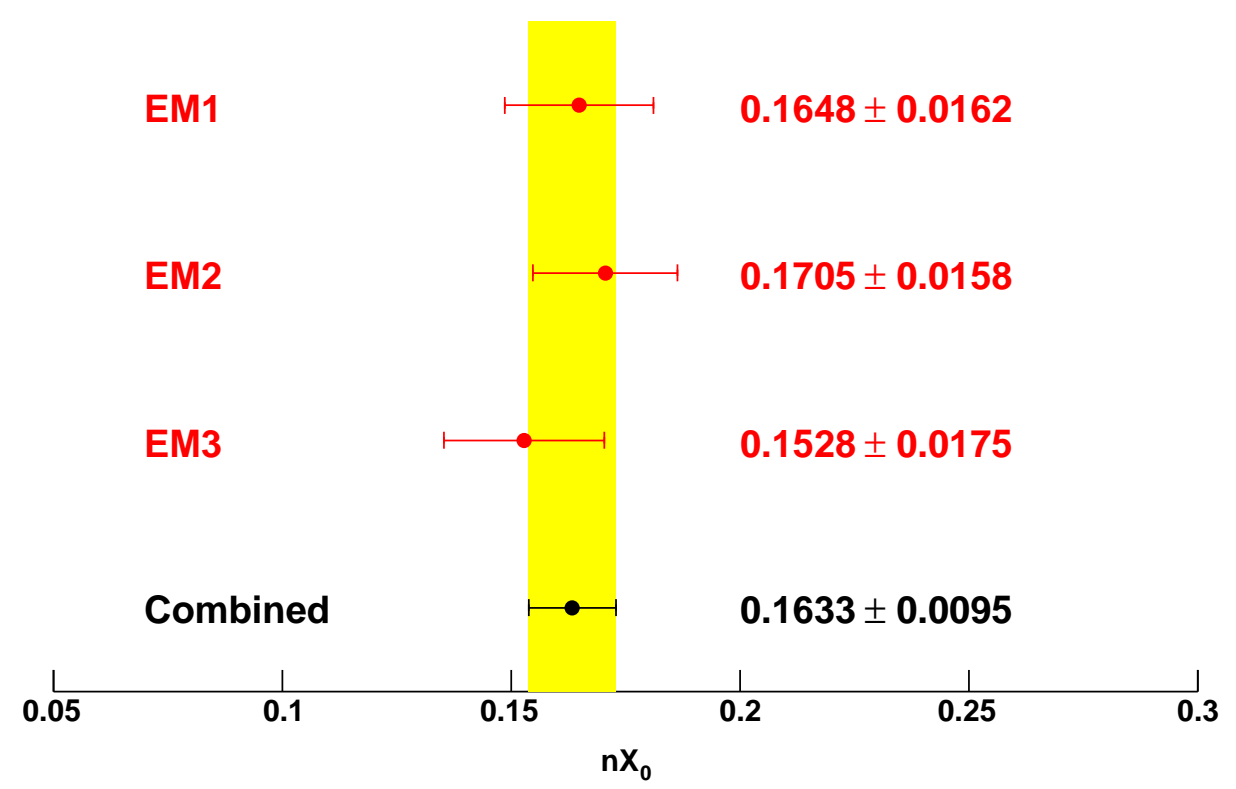

Figure 5.14: Results of fits for missing $X_{0}$ in each EM layer, independently. 


\section{TOYEemf_1_23}

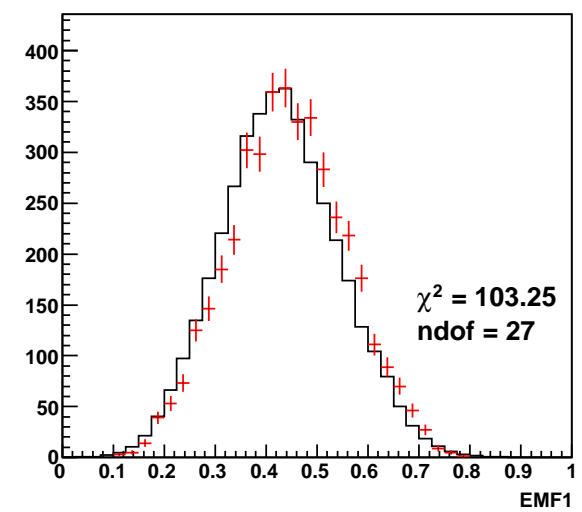

TOYEemf_3_23

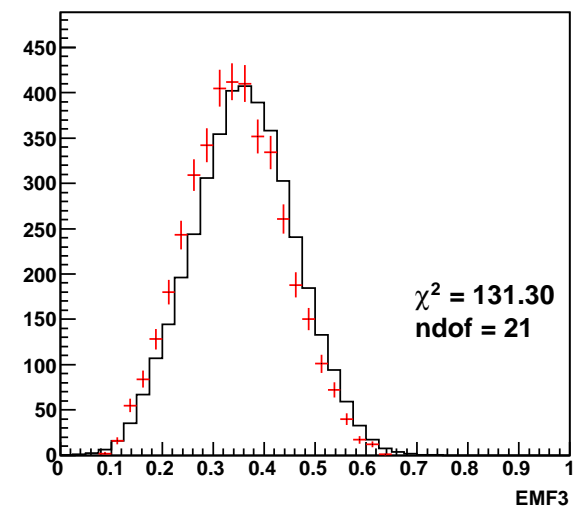

\section{TOYEemf_2_23}

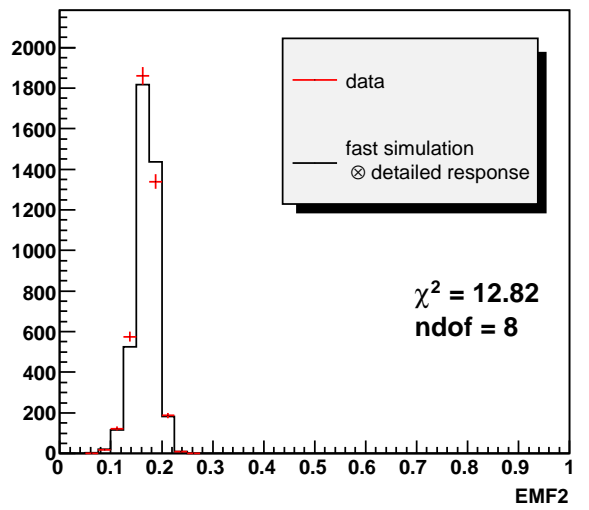

TOYEemf_4_23

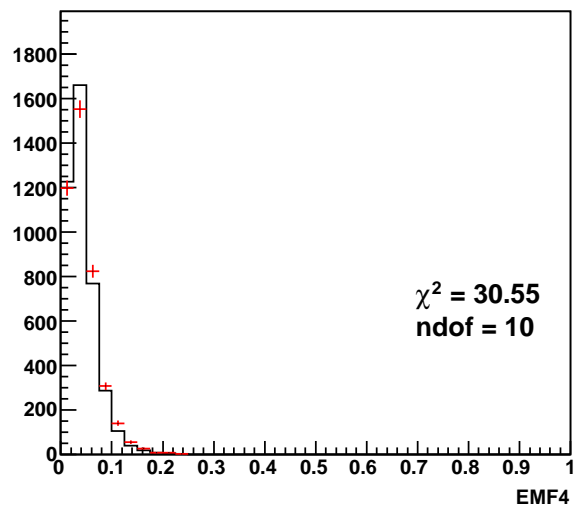

Figure 5.15: Comparison between data and an MC model of the longitudinal shower profile for category $23 Z$ data before missing material is added. 


\section{TOYEemf_1_23}

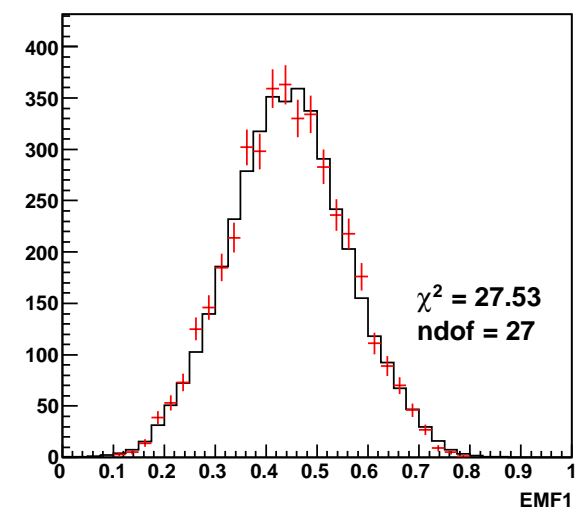

TOYEemf_3_23

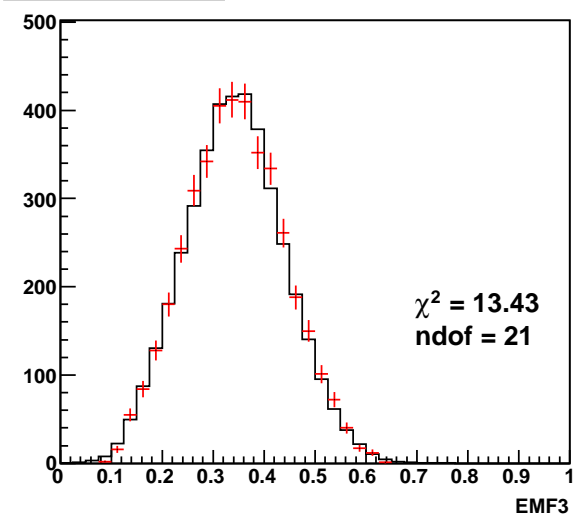

\section{TOYEemf_2_23}

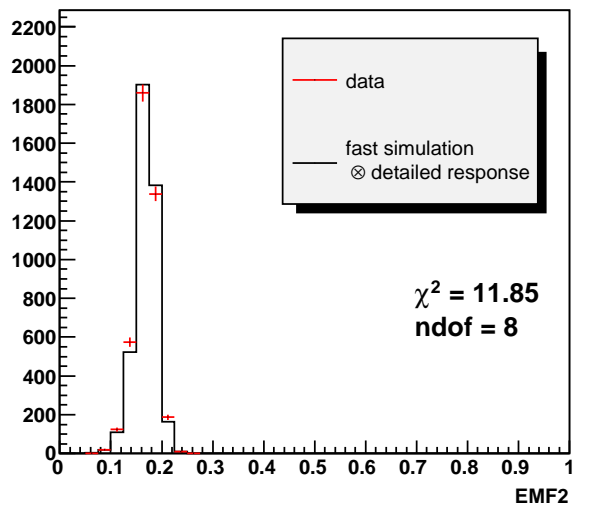

TOYEemf_4_23

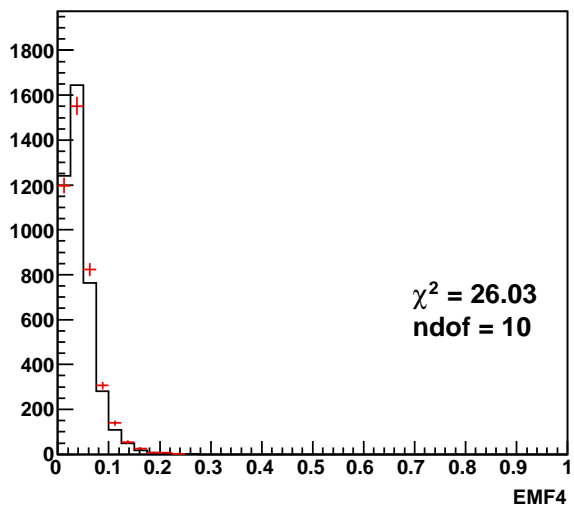

Figure 5.16: Comparison between data and an MC model of the longitudinal shower profile for category $23 Z$ data after missing material is introduced. 


\section{Chapter 6}

\section{Event Selection}

\subsection{Data}

The $\mathrm{W}$ mass and width measurement discussed in this dissertation use data samples from a run period generally referred to as Run IIa. These data were collected between July 26th 2002 and July 22nd 2006, between the run numbers 160582 and 215670, and comprise roughly $1 \mathrm{fb}^{-1}$ of integrated luminosity [67]. Our samples are obtained from the Common Sample Group's "EM Inclusive" skim. In addition to the $W \rightarrow e \nu$ sample used for our W mass and width fits, we heavily rely on a sample of independent, but kinematically similar $Z \rightarrow e e$ events for detector calibration and Monte Carlo tuning, and an "EM + jet" sample to study the probability of a jet faking an electron $[68,69]$.

\subsection{Triggers}

The collision rate of $2.5 \mathrm{MHz}$ produces data at a much higher bandwidth than the $\mathrm{D} \varnothing$ data acquisition system can write to disk. To reduce this rate to manageable levels, we use the three-tiered trigger system to make low-level decisions regarding what collisions are most interesting $[70,71,72,73]$.

The recorded data used in this analysis are collected from events that pass a combination of single EM trigger conditions. As luminosity increased, these conditions had to be modified to accommodate new run conditions. The trigger list for the $W \rightarrow e \nu$ and $Z \rightarrow e e$ data sets is divided into four periods. Table 6.2 shows the trigger definitions used for this analysis. 


\subsection{Track and Vertex Reconstruction}

SMT and CFT hits are used to reconstruct the trajectories of charged particles in the detector, as well as the vertex from which they originate. The quality of a track is determined by a $\chi^{2}$ fit of a track to nearby SMT and CFT hits. The track finding algorithm first looks for track segments in each layer, before matching these segments to a global track.

The interaction points of a given event are called the "event vertices". There are two kinds of event vertex, "primary" and "secondary". The primary vertex, as its name implies, refers to the original point of interaction, and has the largest number of track originating from it. In the $\hat{x}$ and $\hat{y}$ directions, the plane orthogonal to the beam direction, the primary vertex is typically close to the origin, since the cross-section of the beam is small $(\approx 40 \mu m)$. In the $\hat{z}$ direction along the beamline, however, the location of the vertex can vary significantly, with mean $\hat{z}$ component close to zero, but RMS close to $30 \mathrm{~cm}$. A primary vertex must contain at least 3 good tracks, each with at least one hit in the SMT. The secondary vertex is a displaced vertex with fewer tracks, due to the decay of a long live meson such as the $B, K_{s}$, or $D$, and only requires two tracks. Secondary vertices are not used in this analysis. More detailed treatment of tracking at D $\varnothing$ can be found in References $[74,75,76,77]$.

\subsection{EM Reconstruction and Clustering Algorithm}

EM showers are found using a "simple cone" clustering algorithm that searches through a list of the calorimeter trigger towers $(0.1 \times 0.1$ in $\eta \times \phi)$ with the highest EM energy. The EM energy of a tower is defined that the energy in the first four "EM layers", and the fifth, "course hadronic", layer of the calorimeter. The highest energy tower is selected as the seed in a pre-cluster. Adjacent towers above a $50 \mathrm{MeV}$ threshold and within a $5 \times 5$ window in $i \eta \times i \phi$ are added onto the seed tower. The centroid of the cluster is recomputed and used as the new cluster seed. The process is repeated until the centroid becomes stable. The cluster energy is then calculated from all of the cells in the cone. 


\section{$6.5 E_{T}$ Reconstruction}

The raw missing $E_{\mathrm{T}}$ is constructed as a vector sum of all calorimeter cells above the zerosuppression threshold. Once we have identified good EM clusters, electron energy corrections are applied and the $E_{T}$ is recomputed.

\subsection{Particle Identification}

Electrons in our data are identified as isolated showers of EM energy with $p_{\mathrm{T}}>25 \mathrm{GeV}$ and pseudorapidity $\left|\eta_{\text {det }}\right|<1.05$ that conform to shower shape criteria and match a track in our tracking system. In the following section we describe these criteria in more detail.

Electrons must pass a basic isolation condition. For electrons, we require $f_{\text {iso }}<0.2$, where isolation is defined as:

$$
f_{\text {iso }}=\frac{E_{\text {cone }}-E_{\text {core }}}{E_{\text {core }}}
$$

The EM fraction, $f_{E M}$ is defined as the ratio of shower energy in the EM layers of the calorimeter to total energy in the cluster, and is must be above 0.90 for electrons.

The H-Matrix is a shape-based discriminant used to distinguish between electron showers, and those of other particles in the calorimeter. In the central calorimeter, we use a $7 \times 7$ covariant matrix to compare calorimeter showers with those of Monte Carlo electrons in our full detector simulation, based on 7 variables: the fractional energies in the first four EM layers, shower width in the $\eta$ direction, the logarithm of the cluster energy, and location of the primary vertex. The H-Matrix is the inverse of this matrix and gives a $\chi^{2}$ for the comparison between that calorimeter shower and typical full MC electron showers. Thus, the lower the H-Matrix value, the more electron-like the shower. For the CC electrons used in this analysis, we equire $\operatorname{Hmx} 7<12$.

Good electrons must have $p_{\mathrm{T}}>25 \mathrm{GeV}$ and pseudorapidity $\left|\eta_{\text {det }}\right|<1.05$. In addition, we exclude the edges near module boundaries of the calorimeter to avoid edge-effects.

We require the electrons to match hits in SMT and CFT, with track $p_{\mathrm{T}}>10 \mathrm{GeV}$ matched 
to an EM cluster to within 0.05 in eta and phi. The quality of the match is given by

$$
\chi^{2}=\left(\frac{\Delta \phi}{\sigma_{\phi}}\right)^{2}+\left(\frac{\Delta z}{\sigma_{z}}\right)^{2}
$$

Where $\Delta \phi$ and $\Delta z$ are the difference between the electron centroid in the calorimeter and the track extrapolated to the third EM layer, and $\sigma_{z}$ and $\sigma_{\phi}$ are the measured resolutions.

\subsection{Offline Selection}

The final offline selection criteria are given in Tables 6.7 and 6.7. After all selection criteria are applied there are $18,725 Z \rightarrow e e$ events and 499,830 W $\rightarrow e \nu$ events left in the DØ Run IIa sample. 


\begin{tabular}{|l|l|l|l|}
\hline Trigger & L1 & L2 & L3 \\
\hline EM_HI_SH & $\operatorname{CEM}(1,10)$ & $\operatorname{EM}(1,12)$ for runs $>169523$ & ELE_LOOSE_SH_T $(1,20)$ \\
\hline E1_SHT20 & $\mathrm{CEM}(1,11)$ & none & ELE_NLV_SHT $(1,20)$ \\
\hline E1_SHT22 & $\mathrm{CEM}(1,11)$ & $\mathrm{EM}(1,15)$ & ELE_NLV_SHT $(1,22)$ \\
\hline E1_SHT25 & $\mathrm{CEM}(1,12)$ & $\mathrm{EM}(1,15)$ & ELE_NLV_SHT $(1,25)$ \\
\hline
\end{tabular}

L1 triggers

\begin{tabular}{|l|l|}
\hline $\operatorname{CEM}(1,10)$ & one EM trigger tower $E_{T}>10 \mathrm{GeV}$ \\
\hline $\operatorname{CEM}(1,11)$ & one EM trigger tower $E_{T}>11 \mathrm{GeV}$ \\
\hline $\operatorname{CEM}(1,12)$ & one EM trigger tower $E_{T}>12 \mathrm{GeV}$ \\
\hline
\end{tabular}

\section{L2 triggers}

\begin{tabular}{|l|l|}
\hline $\operatorname{EM}(1,12)$ & one EM candidate with $E_{T}>12 \mathrm{GeV}$ (not present for runs below 169523) \\
\hline $\operatorname{EM}(1,15)$ & one EM candidate with $E_{T}>15 \mathrm{GeV}$ \\
\hline
\end{tabular}

\section{L3 triggers}

\begin{tabular}{|l|l|}
\hline ELE_LOOSE_SH_T(1,20) & $\begin{array}{l}\text { one electron with }|\eta|<3.0 \text { and } E_{T}>20 \mathrm{GeV} \text { passing loose requirements } \\
\text { including shower shape }\end{array}$ \\
\hline ELE_NLV_SHT(1,20) & one electron with $|\eta|<3.6$ and $E_{T}>20 \mathrm{GeV}$ passing tight shower shape \\
\hline ELE_NLV_SHT(1,22) & one electron with $|\eta|<3.6$ and $E_{T}>22 \mathrm{GeV}$ passing tight shower shape \\
\hline ELE_NLV_SHT(1,25) & one electron with $|\eta|<3.6$ and $E_{T}>25 \mathrm{GeV}$ passing tight shower shape \\
\hline
\end{tabular}

Table 6.1: Single EM triggers used in this analysis. 


\section{W events must satisfy:}

- Event must have one tight, CC electron;

$-p_{\mathrm{T}}>25 \mathrm{GeV}$

$-\operatorname{Hmx} 8<20$

- EM fraction $>.9 ;$

- isolation $<0.15$

$-\left|\eta_{\text {det }}\right|<1.05$, where $\left|\eta_{\text {det }}\right|$ is evaluated in EM3;

- matching track;

- Event must have corrected MET > $25 \mathrm{GeV}$;

- Event must have a recoil $p_{\mathrm{T}}<15 \mathrm{GeV}$;

- Event must have a transverse mass between $50 \mathrm{GeV}$ and $200 \mathrm{GeV}$;

\section{$\mathrm{Z}$ events must satisfy:}

- Event must have two tight electrons, at least one in the CC;

$-p_{\mathrm{T}}>25 \mathrm{GeV}$

$-\operatorname{Hmx} 8<20$, or $\operatorname{Hmx} 7<12$

- EM fraction $>.9 ;$

- isolation $<0.15$;

- $\left|\eta_{\text {det }}\right|<1.05$, or $\left|\eta_{\text {det }}\right|$ is between 1.5 and 2.3, where $\left|\eta_{\text {det }}\right|$ is evaluated in EM3;

- matching tracks;

- Event must have a recoil $p_{\mathrm{T}}<15 \mathrm{GeV}$;

- Event must have a transverse mass between $50 \mathrm{GeV}$ and $200 \mathrm{GeV}$; 


\section{Chapter 7}

\section{Electron Measurement and Simulation}

In this Chapter we explore those detector effects involved in electron measurement, and the parameterized model we use to simulate them.

In data and full Monte Carlo, reconstructed electrons correspond to clusters of energetic cells in the calorimeter. These clusters cover a $0.5 \times 0.5$ region in $\eta \times \phi$ and the first five calorimeter layers. The precision and accuracy of electron energy measurements is limited by the granularity and finite calibration of the segments in these clusters, as well as contributions from additional activity in the detector.

\subsection{Electron Energy Measurement}

The measured energy of an electron is described by the following equation:

$$
E_{\text {Meas }}=\left(\alpha E_{\text {True }}+\beta\right) \oplus \sigma_{E M}\left(E_{\text {True }}, \eta\right)+\Delta u_{\|}\left(u_{\|}, L\right)
$$

A linear form consisting of an energy scale, $\alpha$ and offset $\beta$ is used to describe the energy response of the detector to an electron at a given true energy. This energy is then smeared with a gaussian resolution $\sigma_{E M}$ that depends on the energy and pseudo-rapidity of the electron. Finally a correction $\Delta u_{\|}$is applied to account for contributions to the measured electron energy from the hadronic recoil and additional activity in the detector.

\subsubsection{Parameterized Energy Response}

We adopt a linear model to describe the overall EM energy response of the calorimeter, which accurately describes the detector behavior as observed in collider and test beam data. The measured energy is related to the true energy by the following equation: 


$$
E_{\text {Meas }}=\alpha E_{\text {True }}+\beta
$$

We tune this model to observables found in $Z \rightarrow e e$ data. One observable that we use is the known location of the $\mathrm{Z}$ mass peak itself. However, since the energy scale and offset are strongly correlated, we need further constraints to separate the two terms. The reconstructed $\mathrm{Z}$ mass distribution ignores the $p_{\mathrm{T}}$ spectra of the constituent decay electrons. Knowing that these electrons are not mono-chromatic, we construct another observable to provide additional information in constraining our scale and offset fit.

$$
f_{Z}=\frac{E_{\text {true }}\left(e_{1}\right)+E_{\text {true }}\left(e_{2}\right)}{M_{\text {true }}(Z)}(1-\cos \theta)
$$

This new observable, $f_{Z}$ relates the mass of the energy scale and offset with both the measured mass and the $p_{\mathrm{T}}$ spectra of the two decay electrons.

$$
M_{o b s}=\alpha M_{\text {true }}(Z)+\beta f_{Z}+O\left(\beta^{2}\right)
$$

The final fit for $\alpha$ and $\beta$ is a 2-parameter fit to minimize the $\chi^{2}$ between both the central values and resolutions of the $\mathrm{Z}$ mass for bins of $f_{Z}$. This profile plot in Fig 7.2 shows an example of the profile plots used in the $\chi^{2}$. Figure 7.1 shows the 2-dimension distribution of $f_{Z}$ versus $m_{Z}$ from which the profile is derived. More details on this measurement can be found in [78].

\subsubsection{Parameterized Energy Resolution}

Traditionally, we describe the fractional resolution of the calorimeter using a parameterization with three primary terms, corresponding to the different contributions to the precision of our EM energy measurement:

$$
\frac{\sigma}{E}=\sqrt{\frac{N^{2}}{E^{2}}+\frac{S^{2}}{E}+C^{2}}
$$

$N$ represents the noise term. This term describes resolution effects due to electronics noise, latent radioactivity from the Uranium, and underlying events. The fractional resolution effects due to 


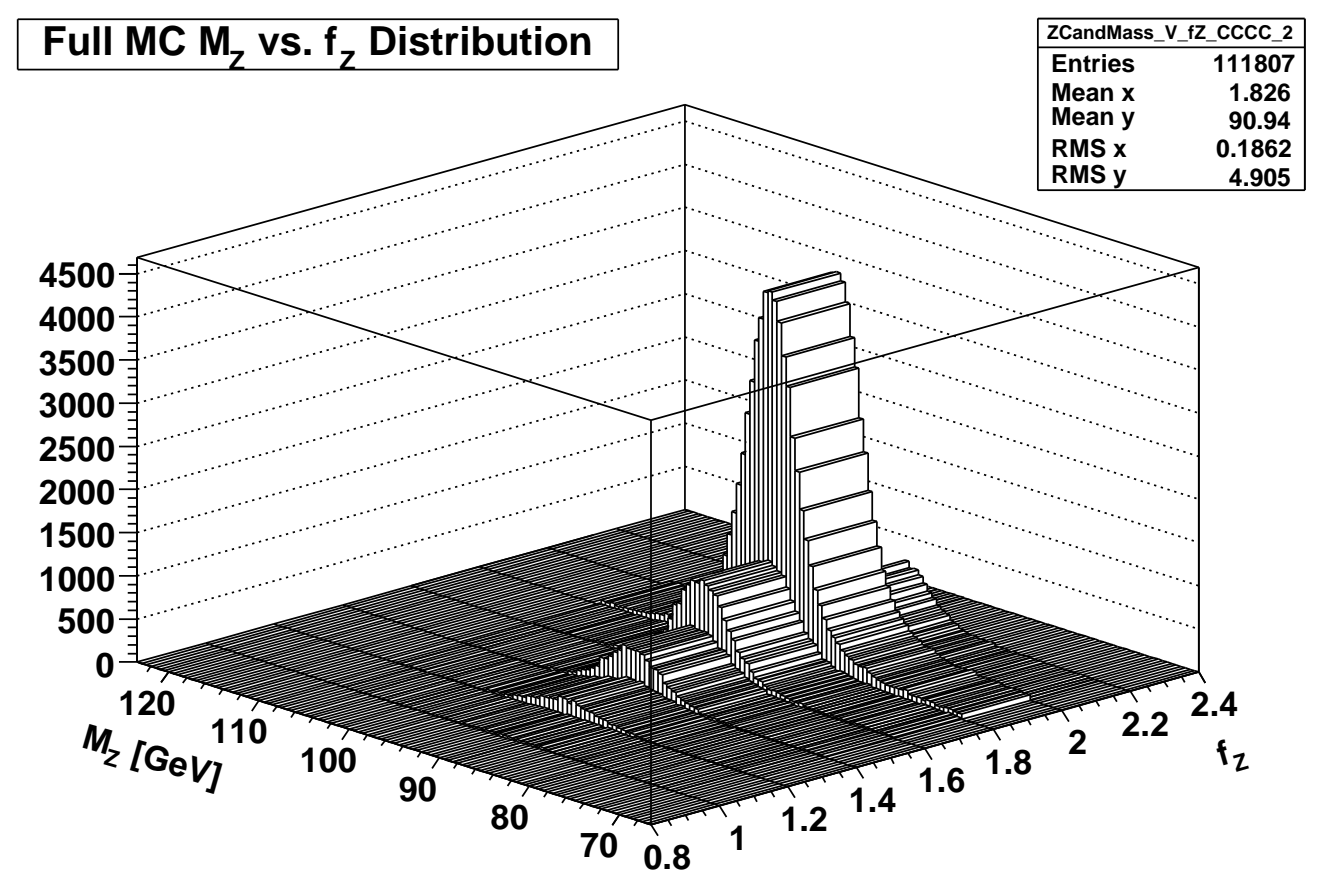

Figure 7.1: The distribution of $M_{\mathrm{Z}}$ vs. $f_{\mathrm{Z}}$ in full Monte Carlo events.

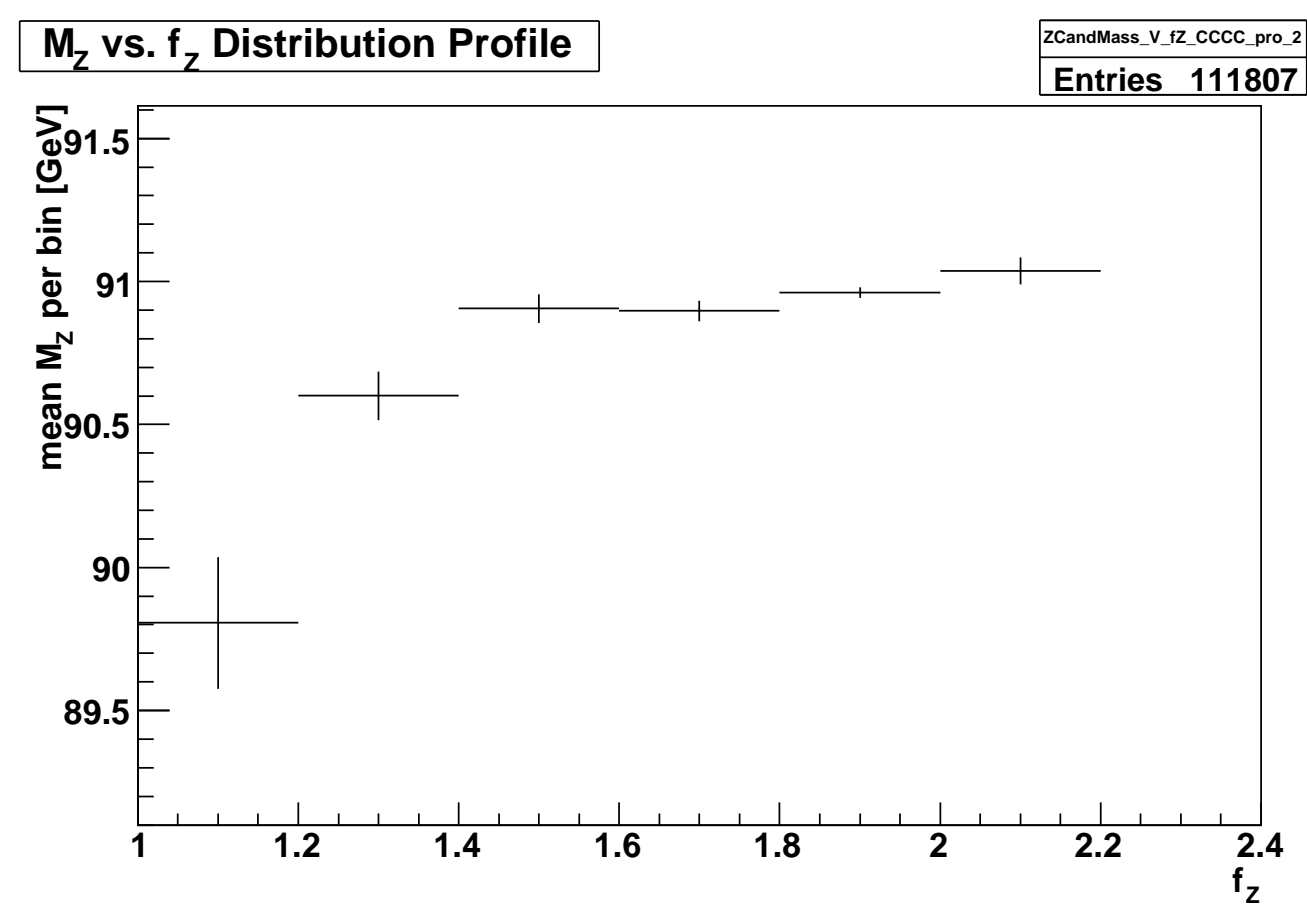

Figure 7.2: The profile plot of $M_{\mathrm{Z}}$ vs. $f_{\mathrm{Z}}$ in full Monte Carlo events. 
the noise term are proportional to $1 / \mathrm{E}$ and are therefore insignificant at high energies. $C$ is the constant term which describes smearing effects due to relative miscalibrations of the calorimeter $\eta-\phi$ segmentations. This term has a constant effect on the fractional resolution, independent of the energy, and is therefore the dominant effect at high energies. $S$ corresponds to the sampling term. Because we only collect only a fraction of the total energy as signal, we are subject to statistical fluctuations in shower development and punch-through of the inactive layers. The sampling term describes the resolution effects due to these fluctuations.

It is important to get the energy dependence of our resolution right, because we tune it at the energy of $Z$ decay electrons and need to extrapolate down to the energy of $W$ decay electrons.

To account for the new dependences of the resolution on incident angle and energy due to new material in front of the detector, we further parameterize the sampling resolution as a function of electron energy and $\eta_{d e t}$, so the formula for fractional resolution becomes:

$$
\frac{\sigma}{E}=\sqrt{\frac{N^{2}}{E^{2}}+\frac{S_{n e w}(E, \theta)^{2}}{E}+C^{2}}
$$

where,

$$
S_{n e w}=S(E, \theta)=\left(\frac{S_{1}}{\sqrt{E}}+S_{2}\right) \frac{e^{S_{e x p} / \sin \theta}}{e^{S_{\text {exp }}}}
$$

and

$$
S_{e x p}=S_{3}-S_{4} / E-S_{5}^{2} / E^{2}
$$

These parameters are tuned to the special full MC sample constrained to $Z \rightarrow e e$ and $J / \psi \rightarrow e e$ data, and depend on the amount of "missing material" in our detector model, as described in Chapter 5. More information on the resolution measurements can be found in [66, 79]. 


\subsubsection{Underlying Energy Corrections}

Some of the energy we attribute to the electron, is not directly related to the lepton; It consists of hadronic energy and other detector activity that overlaps with the calorimeter cells containing the electron. To account for this effect, we must add this "underlying energy" $\left(\Delta u_{\|}\right)$ onto our simulated electrons, after we smear them parametrically. The underlying energy model must account for known dependences on pseudorapidity, luminosity, and $u_{\|}$, the projection of the hadronic recoil along the electron direction. The model should also account for zero-suppression induced correlations when combining the pure electron with underlying energy contributions.

The underlying energy model used in this analysis is built around a combination of data measurements and full MC studies. In $Z \rightarrow e e$ data, we measure the energy in an adjacent $5 \times 5$ (i $\eta \times \mathrm{i} \phi)$ window rotated away from the data electron in $\phi$. This measurement helps to approximate how much activity we expect beneath the electron for that event $\left(\Delta U E_{r o t}\right)$. The $\Delta U E_{\text {rot }}$ for each event is stored in one of $13 \times 13$ array of histograms corresponding to 13 bins in luminosity and 13 bins in $u_{\|}$, the projection of the recoil along the electron. Figure 7.3 shows the distribution of energies in the rotated window over all events. We would expect the mean energy of the $13 \times 13$ histograms to increase for higher luminosity bins, since there will be more additional activity in the detector. Likewise, for higher $u_{\|}$bins we expect to see higher average energies in a window near the electron, since more of the recoil system overlaps with the electron. Figure 7.4 shows plots of $\Delta U E_{\text {rot }}$ as a function of luminosity and $u_{\|}$. The expected dependencies are indeed observed.

$\Delta U E_{\text {rot }}$ is a good first approximation of the energy beneath the electron cone. However, the combined electron and underlying energies will push more cells in the electron cone above the zero-suppression threshold, increasing the electron response and the effective underlying energy. To understand the effect, we use single electron full MC, with and without zero-bias overlay, and with and without zero-suppression. We measure the $\Delta u_{\|}$correction that must be applied beneath an electron cone as a function of $\Delta U E_{\text {rot }}$ and $\eta$.

In the fast $\mathrm{MC}$ model used to generate templates, we chose random $\Delta U E_{\text {rot }}$ values from the appropriate data histograms corresponding to the $u_{\|}$and instantaneous luminosity of each 
simulated event. Full MC derived functions are used to extrapolate $\Delta U E_{r o t}$ to the actual, zerosuppressed $\Delta u_{\|}$to be applied beneath the fast MC electron. More details on this model can be found in $[80,81]$.

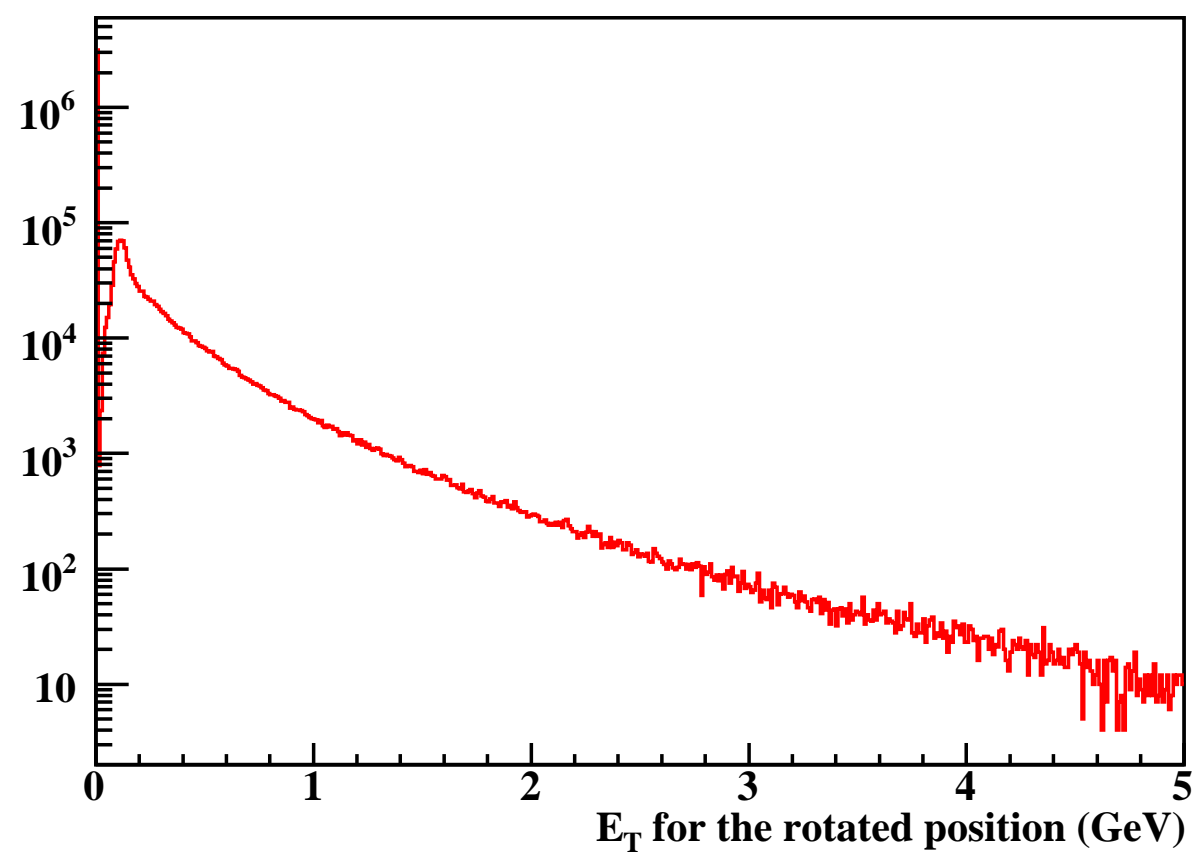

Figure 7.3: Log plot of the energies in the window of towers rotated to a position azimuthally adjacent to the electron window.

\subsection{Electron Position Resolution}

The electron position resolution is described by two parameters in fast $\mathrm{MC}, \sigma_{\eta}$ and $\sigma_{\phi}$ corresponding to the $\eta$ and $\phi$ resolution in the tracking system. These parameters, measured from cosmic muon events to be $\sigma_{\eta}=0.002$ and $\sigma_{\phi}=0.4 \mathrm{mrad}$, are used to smear the generator-level directions of electrons. 

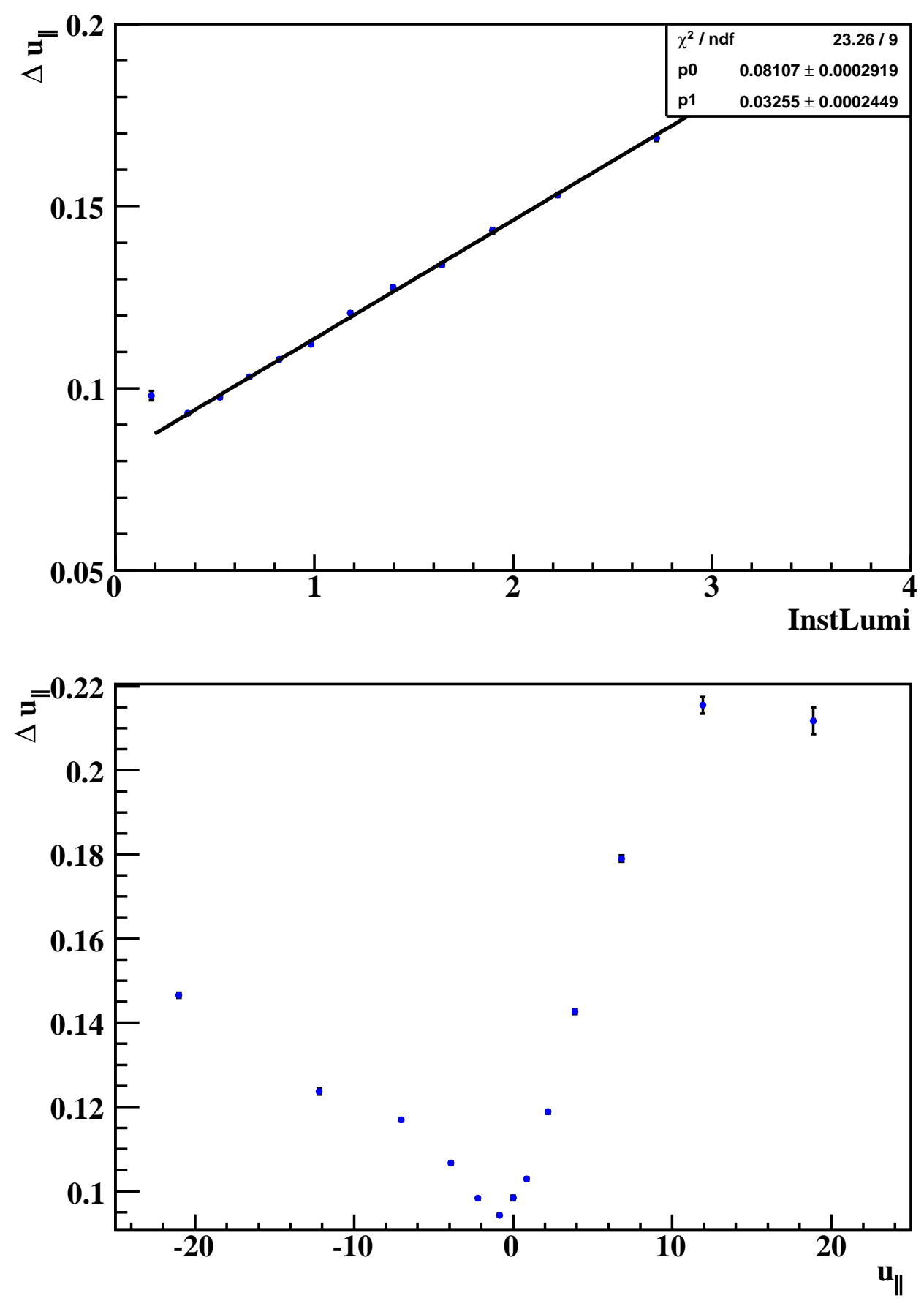

Figure 7.4: Figures showing the dependence on instantaneous luminosity (top) and $u_{\|}$of the underlying energy correction (bottom). 


\section{$7.3 \phi-\bmod$}

The central calorimeter is assembled from 32 separate modules in the azimuthal direction. Gaps in the signal boards and non-uniform electric fields in these boundaries tend to bias the electron clustering algorithm in the calorimeter towards module centers. We reduce this bias with a fiducial cut of $10 \%$ on either side of the module boundaries. This $\phi$-crack cut reduces the overall acceptance in the azimuthal direction by $20 \%$. Nonetheless, some bias still remains and must be accounted for in our electron model.

We describe this bias in terms of a variable called $\phi_{\text {mod }}$, which is given by:

$$
\phi_{\text {mod }}=\bmod (32 \phi / 2 \pi) .
$$

which varies from 0 to 1 . A $\phi_{\text {mod }}$ value of 0.5 corresponds to the center of the $\phi$ modules and a $\phi_{m o d}$ of 0 or 1 corresponds to module boundaries. We determine the bias by measuring the difference between the position of the matching track extrapolated from collision point, and the cluster center of the calorimeter as measured in the EM3 layer. This difference is called the $\phi_{\text {mod }}$ shift. Figure 7.5 shows a scatter plot of the $\phi_{\text {mod }}$ shift versus $\phi_{m o d}$ for $Z \rightarrow e e$ electrons in the calorimeter. Figure 7.6 shows the equivalent profile plot. This bias is applied to the fast MC model [81].

\subsection{Final State Photon Merging}

Decay electrons can emit a photon in the final state. Low energy photons fail to penetrate the calorimeter and their energy is lost from the electron. However, if the radiated photon has sufficient energy to reach the calorimeter, one of three things can happen, depending on the energies of the photon and electron, as well as the proximity of the photon, given by:

$$
\Delta R(e \gamma)=\sqrt{[\phi(e)-\phi(\gamma)]^{2}+[\eta(e)-\eta(\gamma)]^{2}},
$$

If the photon is sufficiently near to the electron, the two EM particles are seen as a single EM 


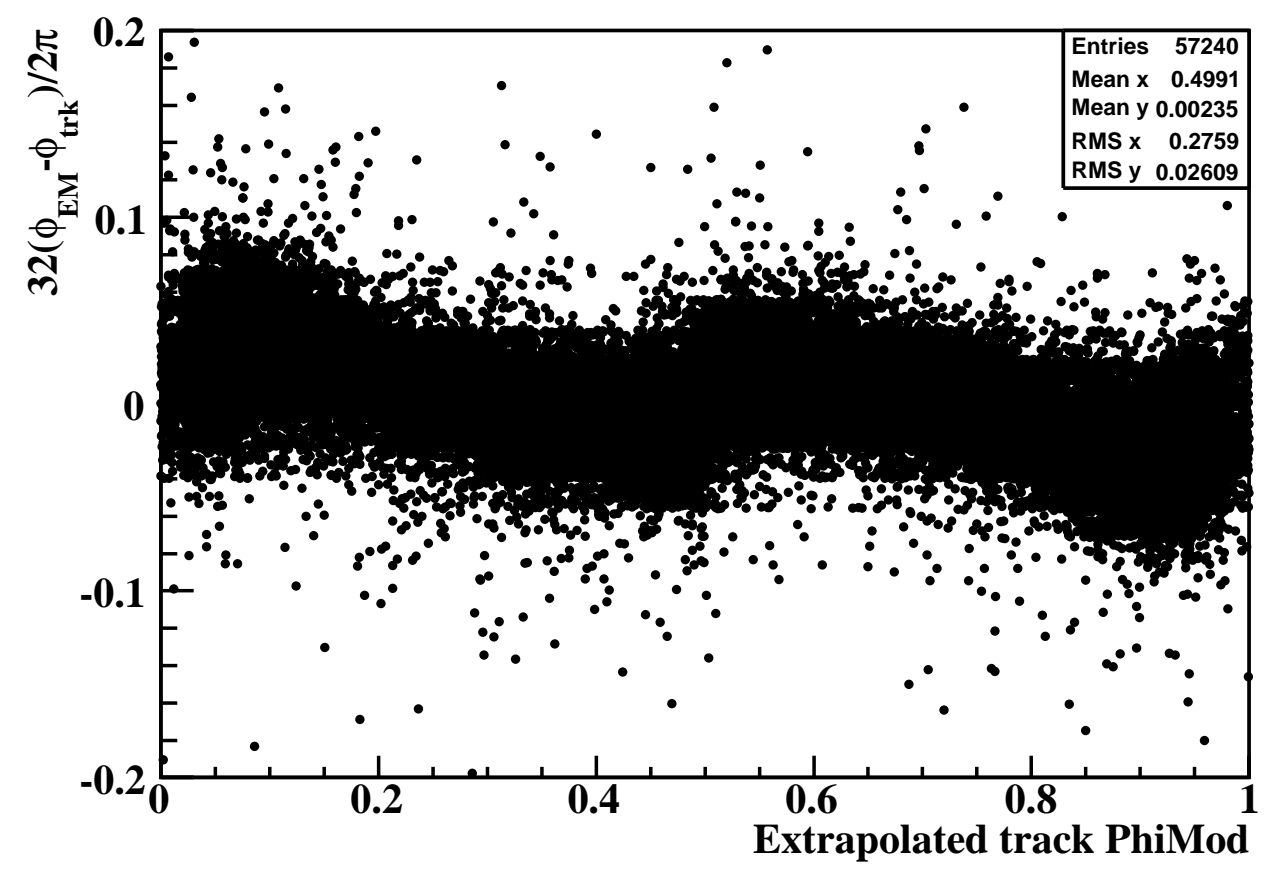

Figure 7.5: Scatter plot of the shift between track position and calorimeter cluster center as a function of extrapolated track $\phi$-mod. 


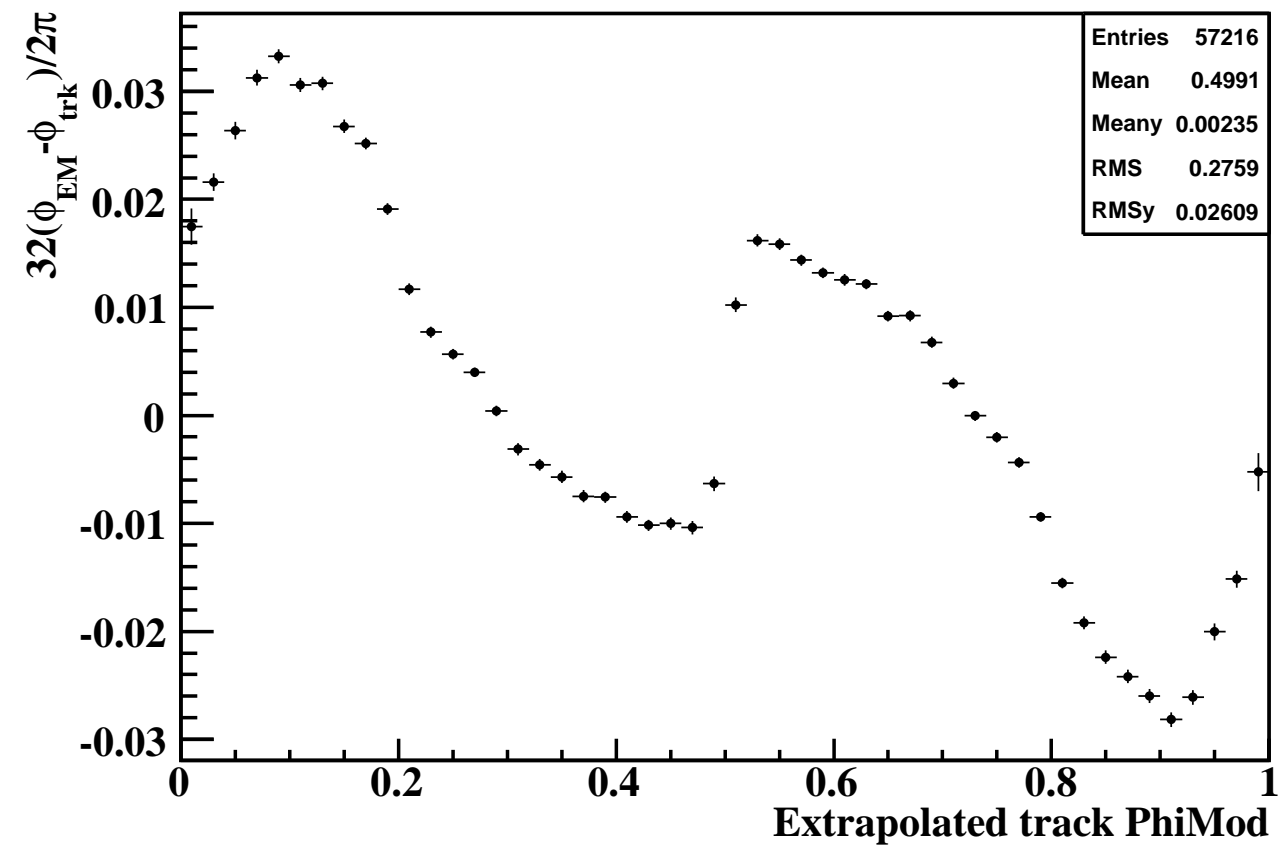

Figure 7.6: Profile plot of the shift between track position and calorimeter cluster center as a function of extrapolated track $\phi$-mod. 
cluster corresponding to the electron. In this case the reconstructed electron energy reflects the merged energy of both the electron and the photon, which is equivalent to the energy of the electron as it would have been reconstructed if it did not radiate a photon. If the photon is sufficiently far from the electron, it is no longer reconstructed as part of the electron energy but instead added on to the measured recoil energy. For intermediate distances between the photon and electron, the energy of the reconstructed photon can interfere with the isolation and shape-based electron selection cuts, and the event is thrown away.

Our final state radiation (FSR) model must therefore not only describe the QED effects at the four-vector level, but also detector effects on the measured kinematics. It must describe the probability of photons penetrating the calorimeter, the probability that the photon and electron will be merged, and the probability that the radiated photon will interfere will interfere with selection cuts and kill the event.

The probability of a photon penetrating the calorimeter comes from the well know properties of high energy photons passing through matter.

Figure 9.1 shows the electron ID efficiency for bins of $\Delta R(e \gamma)$ as a function of the fraction of the electron energy carried by the leading FSR photon $\left(\operatorname{Efrac}_{\gamma}\right)$. The features of these overall efficiency plots are the result of effects from several different selection criteria. The first several bins (corresponding to a very close photon) are driven by the track matching requirement. For the very distant photons of the last few bins, the FSR does not directly interfere with selection criteria, except that for large $\operatorname{Efrac}_{\gamma}$, so much of the electron energy is lost to the photon that it begins to fail basic $p_{\mathrm{T}}$ cuts. The complicated features for the intermediate $\Delta R(e \gamma)$ bins are driven by the HMx shower shape requirement, which was optimized for single electrons and not electrons with nearby photons.

We correct the electron energy by removing the fraction of FSR photon energy lost. This fraction, $\kappa$ is defined as

$$
\kappa=\frac{\left(E_{\text {reco }}[\text { withFSR }]-E_{\text {reco }}[n o F S R]\right) /\left(E_{\text {true }}[n o F S R]\right)}{E \operatorname{frac}_{\gamma}}
$$


Figure 7.8 shows the $\kappa$ fraction as a function of $\mathrm{Efrac}_{\gamma}$ for different bins in $\Delta R(e \gamma)$. As we would expect, for large $\Delta R(e \gamma), \kappa=-1$, since all of the photon energy is lost from the electron.

\subsection{Primary Vertex Simulation}

In order to calculate the $\eta_{\text {det }}$ of a given electron, we need to know both $\eta_{\text {phys }}$ and the position of the vertex. Our fast MC method for generated template distributions must model the vertex distribution observed in data. The shape of the vertex distribution depends on the shape of the luminous region, which varies as a function of hardware changes and instantaneous luminosity. We therefore divide the data into 15 epochs that track major changes over time. The vertex distribution in zerobias data from each epoch is fit with a 3-parameter formula. These 15 sets of three parameter functions are used to describe the vertex distribution in fast $\mathrm{MC}$ as a function of run number [83].

\subsection{Luminosity and Run Number Simulation}

The $\Delta u_{||}$corrections depend on the instantaneous luminosity, and the vertex model depends on run number. In fast MC, the luminosity and run number are randomly chosen from a 2dimensional histogram storing the luminosities and run numbers of of minbias events taken over the same run period as the $W$ and $Z$ data. 

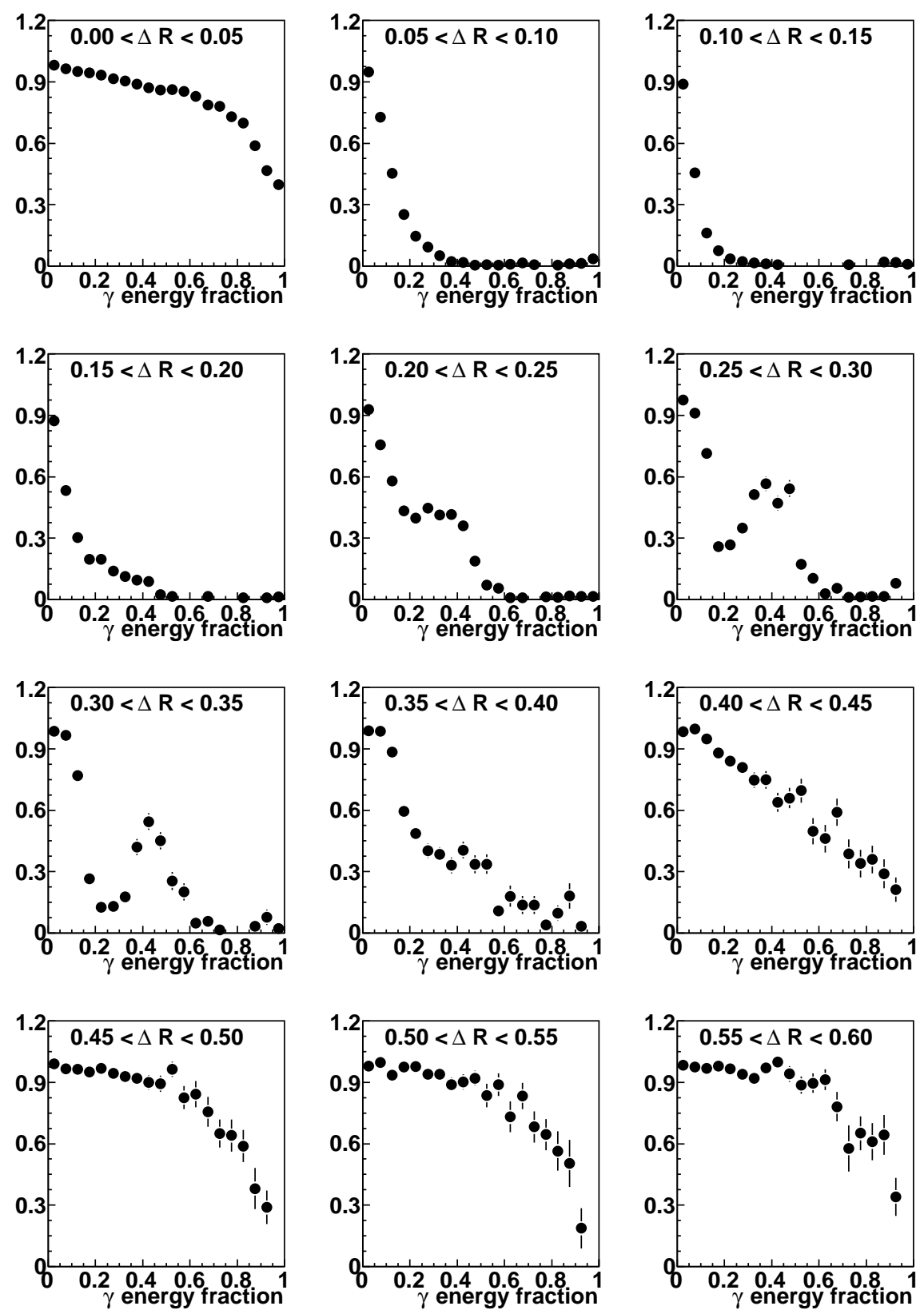

Figure 7.7: The selection efficiencies for electrons in FSR events as a function of the fraction of electron energy carried by the leading photon, for bins in $\Delta R(e \gamma)$, proximity of the photon. 

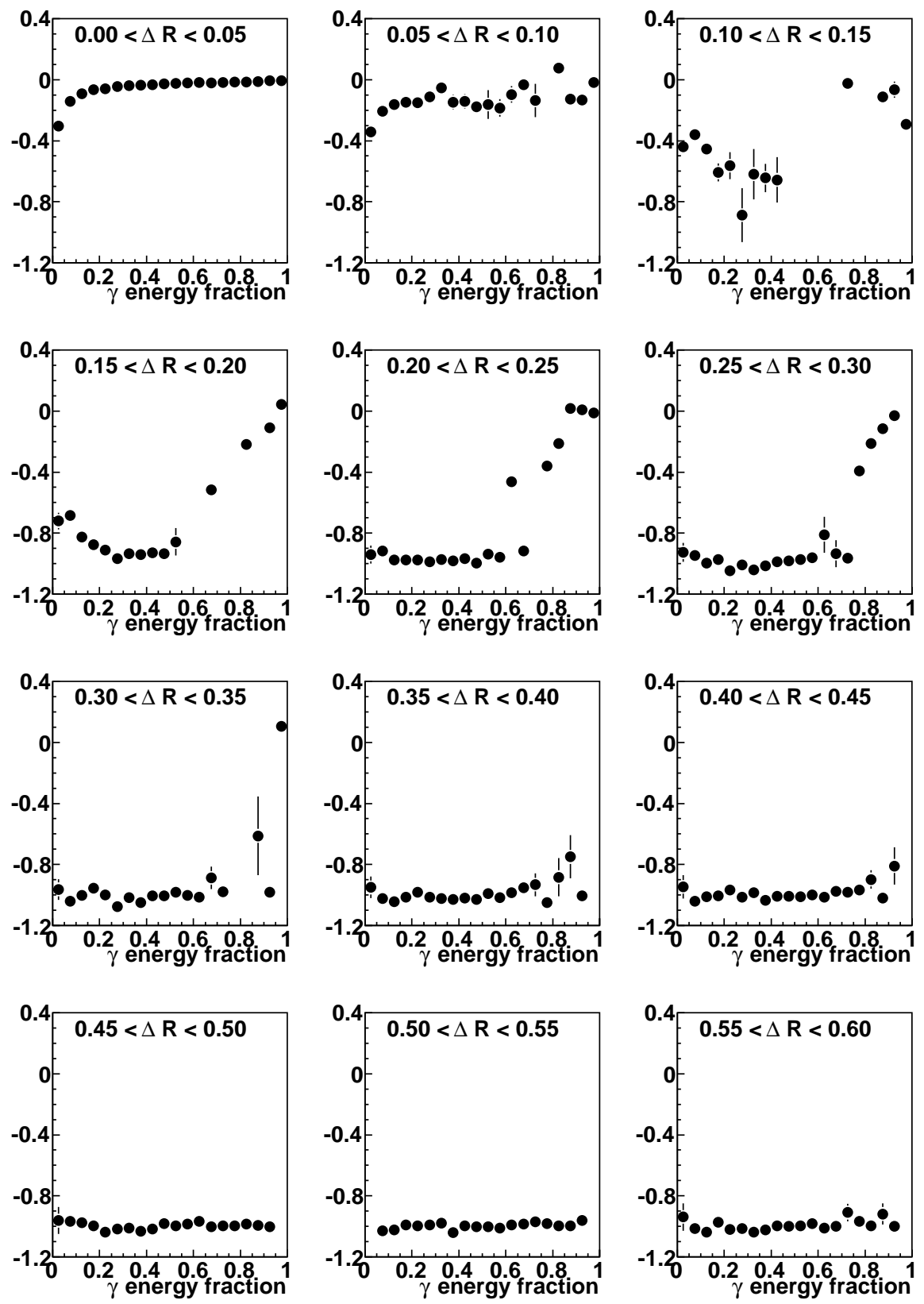

Figure 7.8: The fraction of FSR photon energy lost from the electron as a function of $\mathrm{Efrac}_{\gamma}$ for different bins in $\Delta R(e \gamma)$ 


\section{Chapter 8}

\section{Efficiencies}

Beside the geometric and kinematic cuts imposed on the data, it is necessary to model the electron identification (ID) efficiencies. Absolute inefficiencies in our selection of good $W \rightarrow e \nu$ events do not affect the measurement of the $W$ boson mass and width, except by reducing the statistical power. Rather, our concern is with efficiencies that affect the shape of the relevant distributions by introducing additional dependencies on kinematic variables such as $p_{\mathrm{T}}(e)$, or $\eta_{\text {phys. }}$. In this section we discuss electron ID efficiencies.

\subsection{Trigger Efficiency}

We derive separate efficiencies for the four trigger periods used in this analysis using the "tag-and-probe" method [84]. We take $Z \rightarrow e e$ where one electron (the "tag") is required to pass all of our strict selection cuts and the other electron (the "probe") need only pass a loose requirement that is uncorrelated with the selection criteria being tested. We then study whether the probe electron passes the particular selection cut being tested. Figure 8.1 shows the trigger selection efficiencies for the four different trigger periods.

\subsection{Preselection Efficiency}

Preselection is defined as the efficiency for an EM cluster passing isolation (0.15) and EM fraction (0.9) cuts. The preselection is also measured using tag-and-probe, where the probe is only required to match a track with track $p_{\mathrm{T}}>15 \mathrm{Gev}$. The resulting efficiency, shown in Fig 8.2 as a function of $\eta_{\text {det }}$, is almost flat over the region used in this analysis $\left(\left|\eta_{\text {det }}\right|<1.05\right)$. 

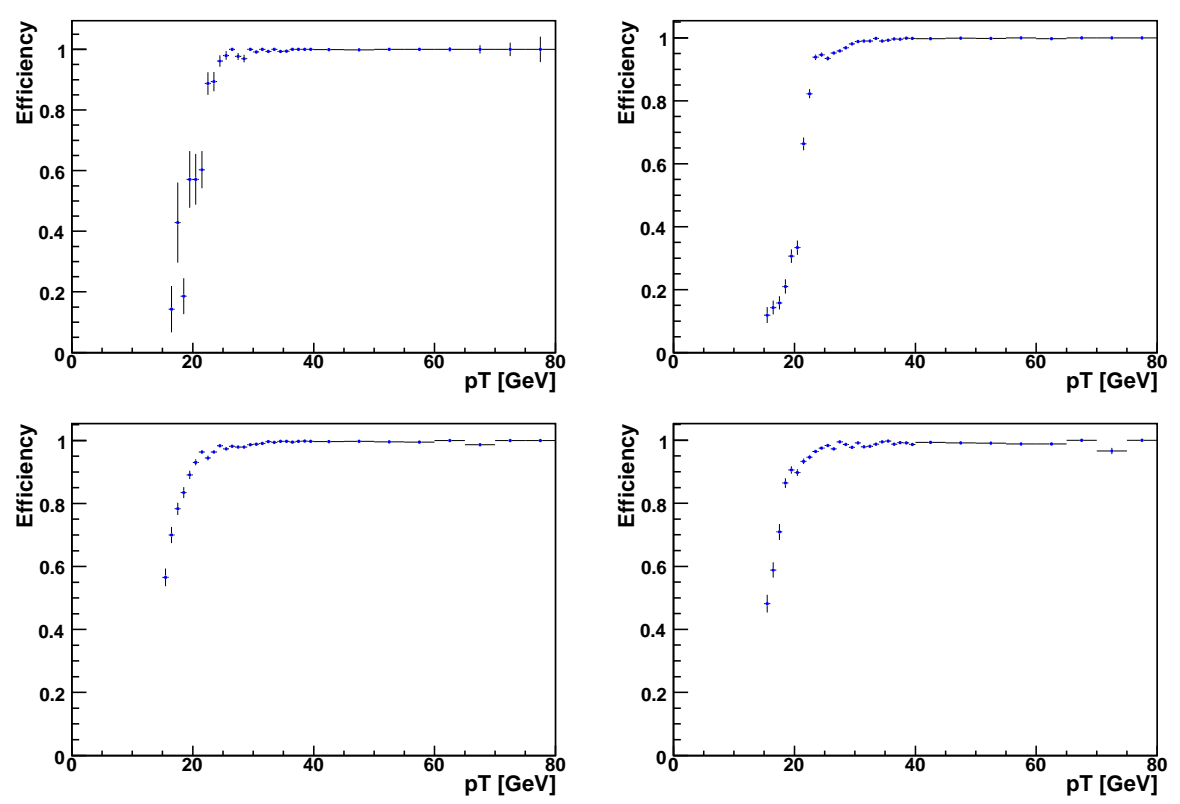

Figure 8.1: The trigger efficiency versus electron $p_{\mathrm{T}}$ for four different run trigger periods: v11 (upper left), v12 (upper right), v13 (lower left, and v14 (lower right).

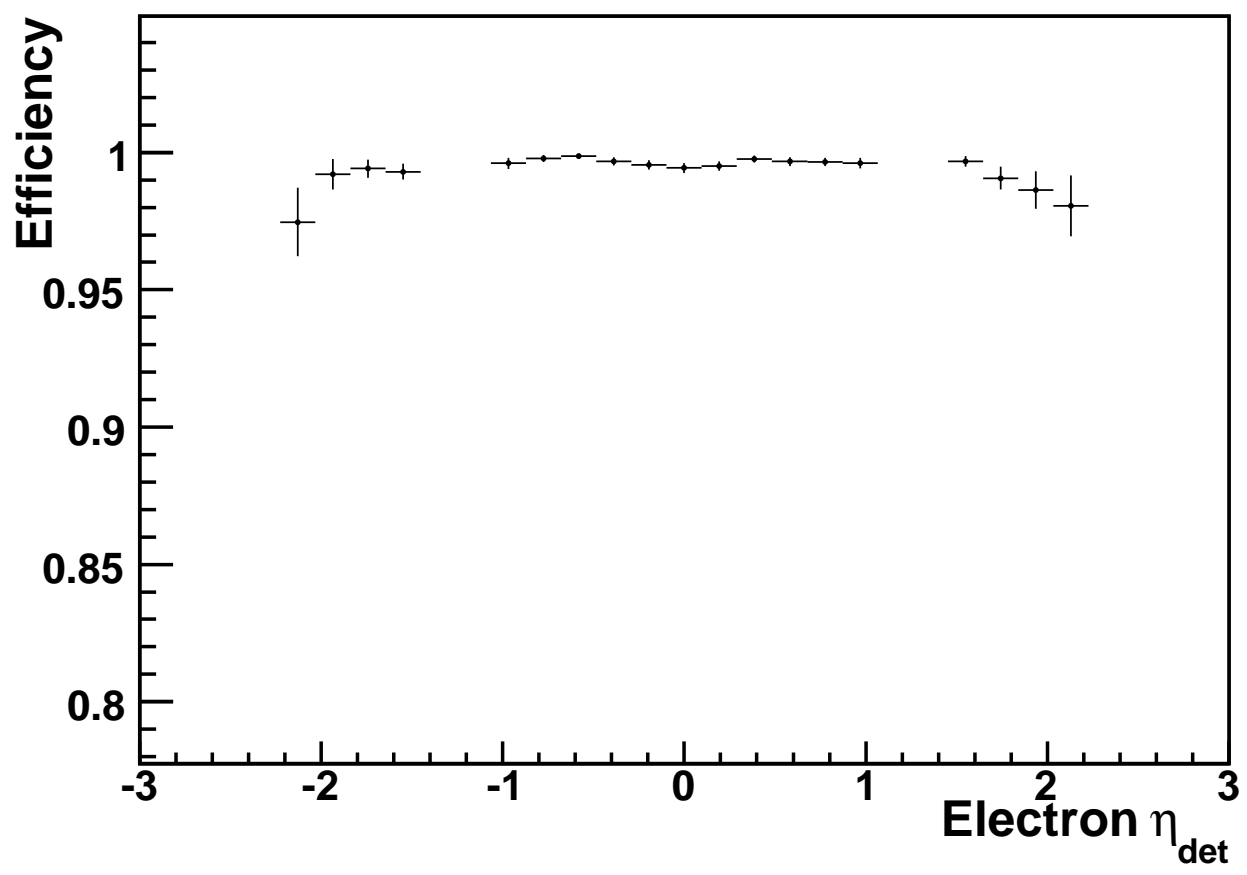

Preselection efficiency as a function of $\eta_{\text {det }}$. 


\subsection{Track Matching Efficiency}

The track matching efficiency is determined from data, again using the tag-and-probe method, as a function of $\eta_{\text {phys }}$, vertex position, and electron $p_{\mathrm{T}}$. Figure 8.2 shows this efficiency as a function of vertex and $\eta_{\text {det }}$. The large drop in efficiency at $40 \mathrm{~cm}$ is due to the requirement that electron match tracks observed in the SMT system.

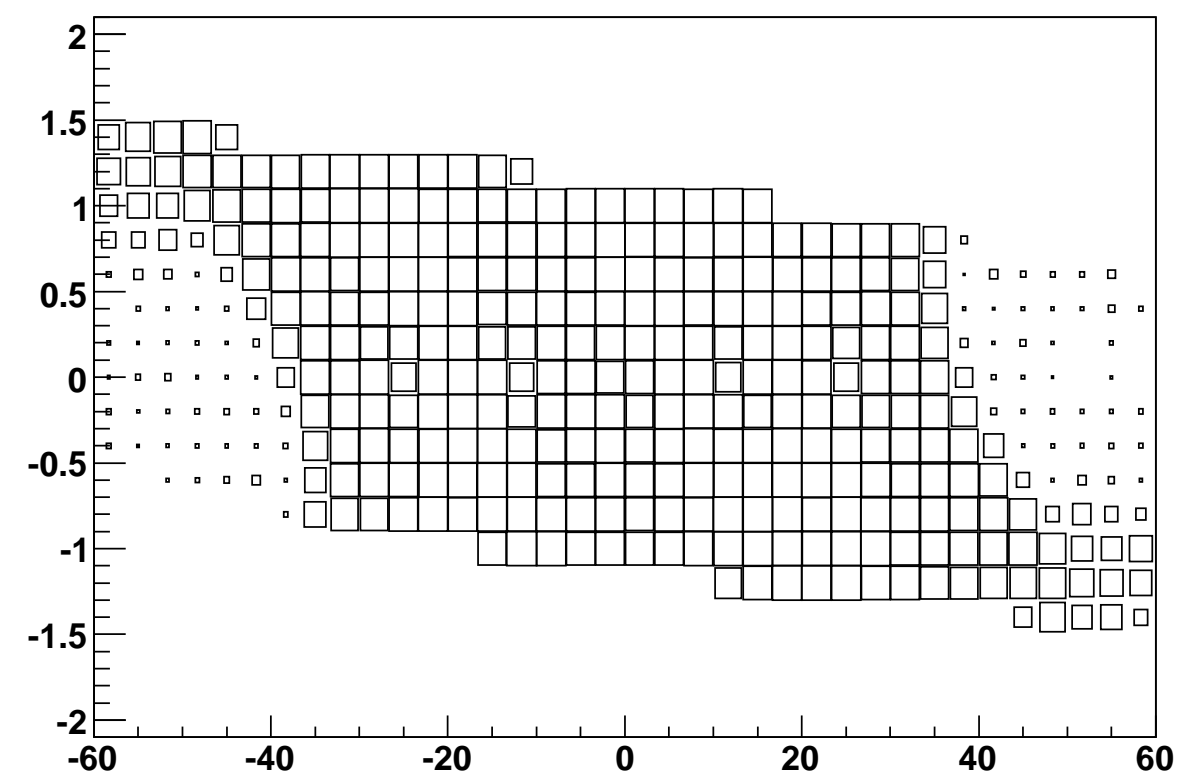

Figure 8.2: The tracking efficiency as a function of $\eta$ and vertex $z$ position shown as a lego plot (left) and a box plot (right)

\subsection{EMID (Hmatrix) Efficiency}

Once an EM cluster has passed the preselection cuts, shower-shape cuts are made on the Hmatrix (described in Chapter 6). The $\eta$ dependence of the Hmatrix efficiency is measured using tag-and-probe. The $p_{\mathrm{T}}(e)$ dependence is determined using a sample of single-electron full MC, taking the ratio of electrons that pass all ID cuts to those electrons that pass preselection [?]. These efficiencies are shown in Fig 8.3 and used as inputs to the fast MC model. 
Of course the full MC sample used to measure the Hmatrix efficiency consists of lone electrons. In $Z \rightarrow e e$ and $W \rightarrow e \nu$ events the presence of other activity in the detector - particularly hadronic activity from the recoil system - interfere with the Hmatrix efficiency. These effects are accounted for in our $u_{\|}$and SET efficiency models, described in the following sections.

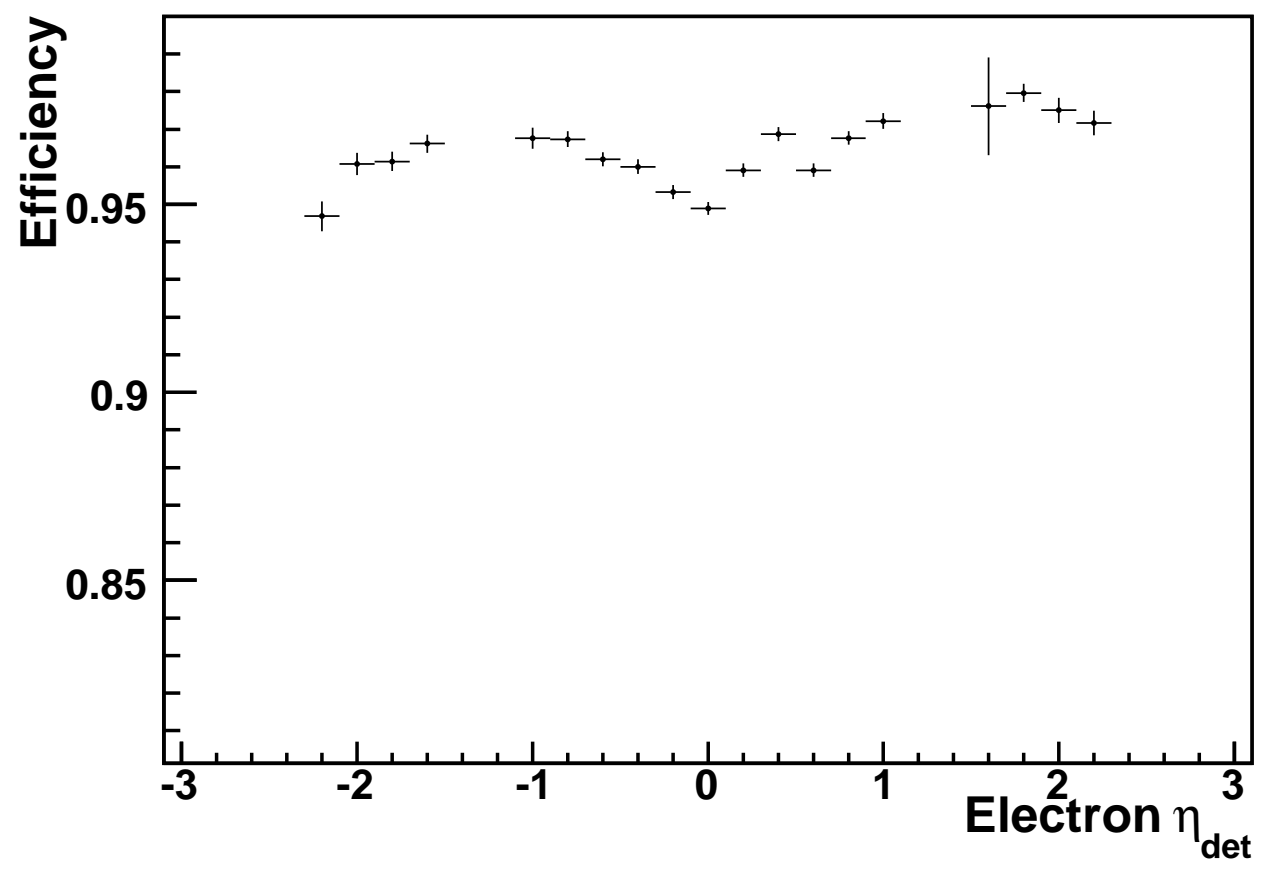

Figure 8.3: The Hmatrix efficiency versus $\eta_{\text {det }}$

\section{$8.5 \phi_{\text {mod }}$ Efficiency}

In addition to the observed $\phi_{m o d}$ shift, described in Chapter 7 , the selection efficiency for electrons decreases close to $\phi$-module boundaries. We determine this efficiency with tag-and-probe studies, using extrapolated track positions to determine the proximity of the electrons to module boundaries. Figure 8.4 shows the measured $\phi_{m o d}$ efficiency. 


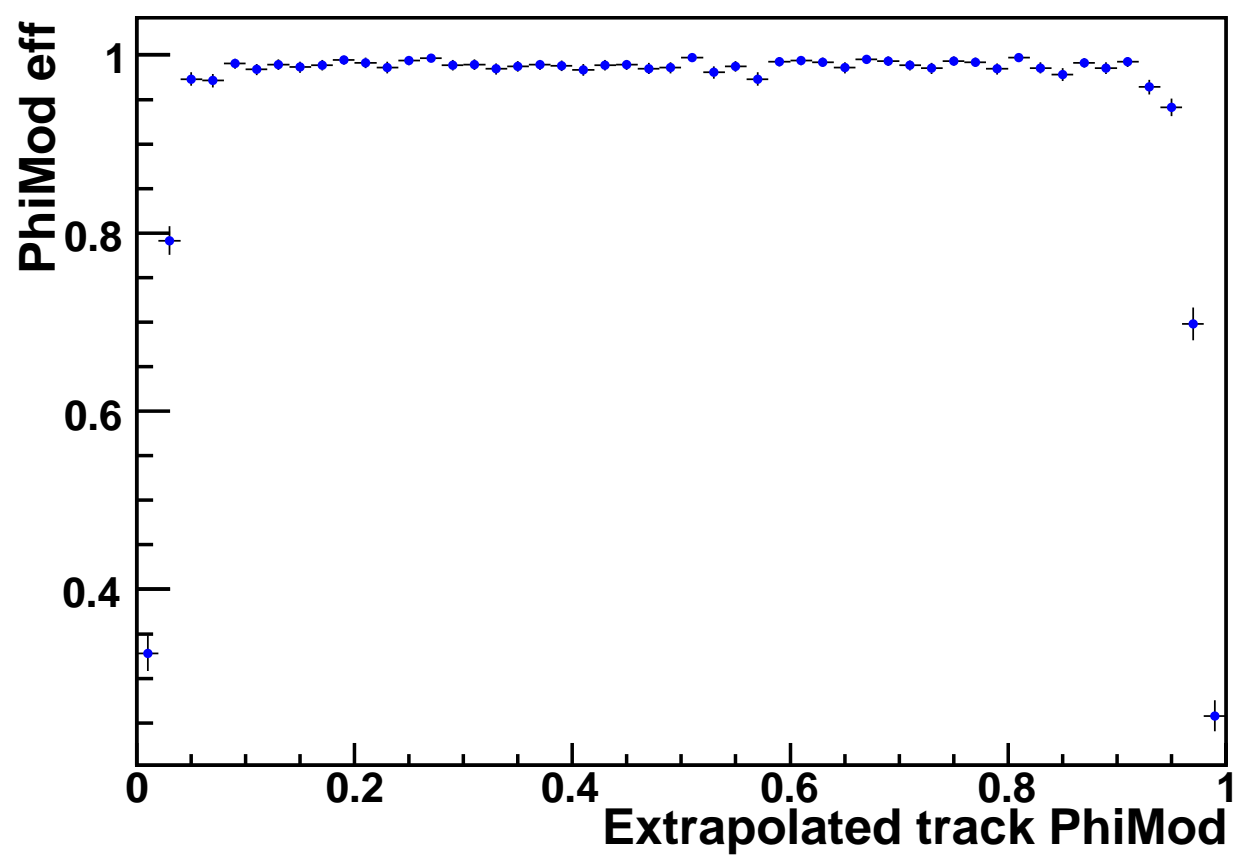

Figure 8.4: The distribution of $M_{\mathrm{Z}}$ vs. $f_{\mathrm{Z}}$ in full Monte Carlo events.

\section{$8.6 u_{\|}$Efficiency}

Electron reconstruction and identification is significantly impacted by the proximity of the recoil to the electron. The larger the quantity of hadronic energy that lies along the direction of an electron, the less likely that electron will pass electron isolation or shower-shape requirements. This effect is modeled in the parametric efficiency model as a function of $u_{\|}$the projection of the recoil along the electron direction.

The $u_{\|}$efficiency is described by a plateau and a negatively sloping line that meet a "kinkpoint" on the $u_{\|}$axis. For $u_{\|}$values below the kink-point, the recoil and electron are spatially separated enough that the electron id efficiency is unaffected by the recoil. After the kink-point, electron selection efficiency drops as the overlap between the electron and recoil increases. The absolute level of the plateau is not important, as it has no bearing on the shapes of relevant $W$ boson observables. The location of the kink point is taken directly from truth-level full MC studies. However, the slope of the $u_{\|}$efficiency is affected by the SET efficiencies and FSR, which we model 
independently in fast MC. Therefore, it cannot be extracted directly from full MC and we must proceed as follows. Once we fix the SET efficiency model (described in the next section), FSR model, and kink point, the slope of the $u_{\|}$efficiency is adjusted so that the overall $p_{\mathrm{T}}$ dependent efficiency between full MC and fast MC match. The fitted slope is roughly one-third of the slope measured directly from full MC. We call this process "double-counting removal", since we are removing the common $p_{\mathrm{T}}$ dependences between the independent SET, $u_{\|}$, and EMID efficiency models in the fast MC. Both the kink-point and slope are measured independently for $W$ and $Z$ events [85].

\subsection{SET Efficiency}

There is an observed discrepancy between the scalar $E_{\mathrm{T}}$ spectra in data and full MC that cannot be modelled by the other components of the efficiency model. This discrepancy suggests an unmodeled electron selection dependence on the scalar $E_{\mathrm{T}}$ (SET), which quantifies the total hadronic activity in the detector. The SET efficiency describes the probability of electron identification as a function of all non-leptonic energy in the detector. With more hadronic activity in the detector, the probability of finding a good electron will diminish.

The overall SET efficiency is derived in full MC $Z \rightarrow e e$ events, exploiting truth information, to obtain an overall event reconstruction efficiency as a function of SET. To whatever extent the full MC does not properly model the SET spectrum in data, tag-and-probe based data corrections are expected to fix the model. These corrections will be described in the next sub-section. Figure 8.6 shows the overall SET efficiency as a function of scalar $E_{\mathrm{T}}$. The SET efficiency differs for $Z \rightarrow e e$ and $W \rightarrow e \nu$ events, since the $Z \rightarrow e e$ data require two good electrons.

One would expect this effect to be more severe for low $p_{\mathrm{T}}$ electrons than for those with high $p_{\mathrm{T}}(e)$, since the electron energy is less significant compared to the background activity. This effect is particularly important for the $\Gamma_{W}$ measurement, since events in the tail of the $M_{\mathrm{T}}$ distribution typically have higher average SET, but also have higher electron $p_{\mathrm{T}}$ spectra. An overall SET efficiency with no consideration for electron momenta would model the tail less efficiently than one 

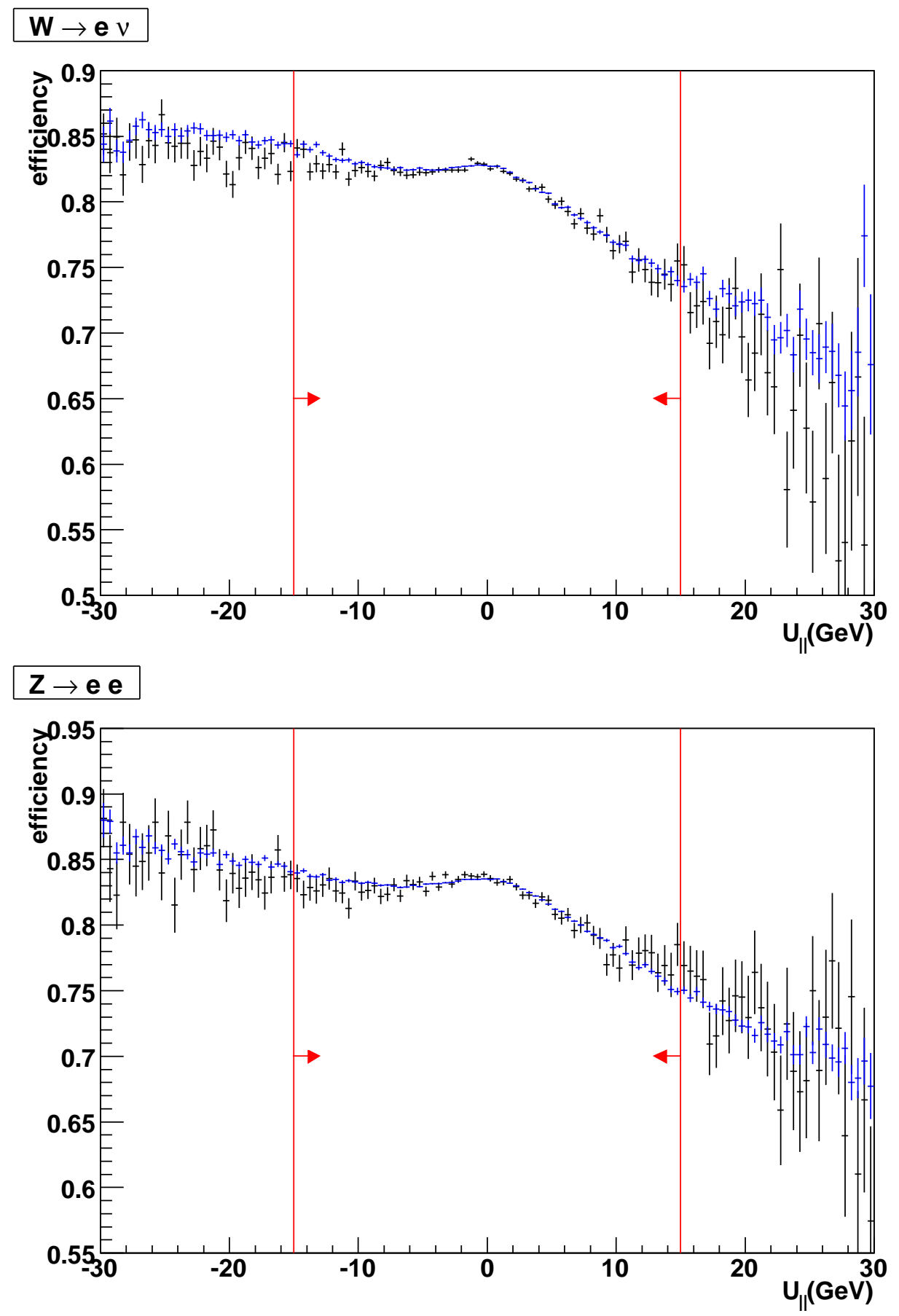

Figure 8.5: The $u_{\|}$efficiency for $Z \rightarrow e e$ (top) and $W \rightarrow e \nu$ (bottom) events for full (black points) and fast (blue points) MC. Good agreement is found. 


\section{SET efficiency}

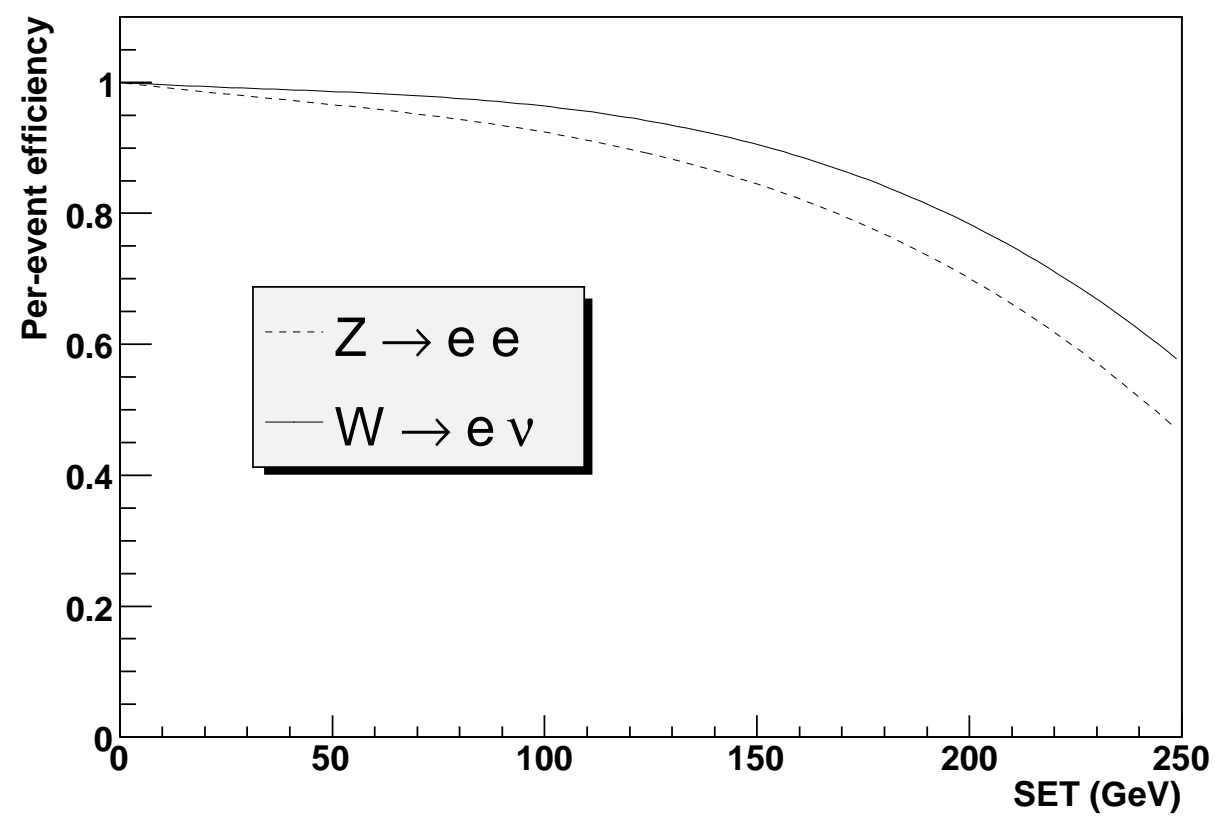

Figure 8.6: The overall reconstruction efficiency as a function of SET for $W$ and $Z$ events.

would expect in data. Corrections based $p_{\mathrm{T}}(e)$ are applied to modify the overall SET efficiency. These corrections are derived from polynomial fits to comparisons between fast and full MC for bins of electron $p_{\mathrm{T}}$ bins. Figure 8.7 shows these correction functions. Overall normalization factors are applied to the respective $p_{\mathrm{T}}(e)$ bins, so that the overall SET efficiency averaged of the $p_{\mathrm{T}}(e)$ spectrum remains the same with the perturbations applied.

The shape of the perturbation functions visibly changes for different $p_{\mathrm{T}}(e)$ bins and can be explained by competing effects between the soft and hard recoil components of the SET. Higher $p_{\mathrm{T}}$ electrons tend to come from more boosted boson decays. Because these events have a large boost, a more significant component of the SET is due to the hard recoil. But, since the hard recoil is more back to back with the electron, that scalar energy is less likely to interfere with the selection of the electron. 


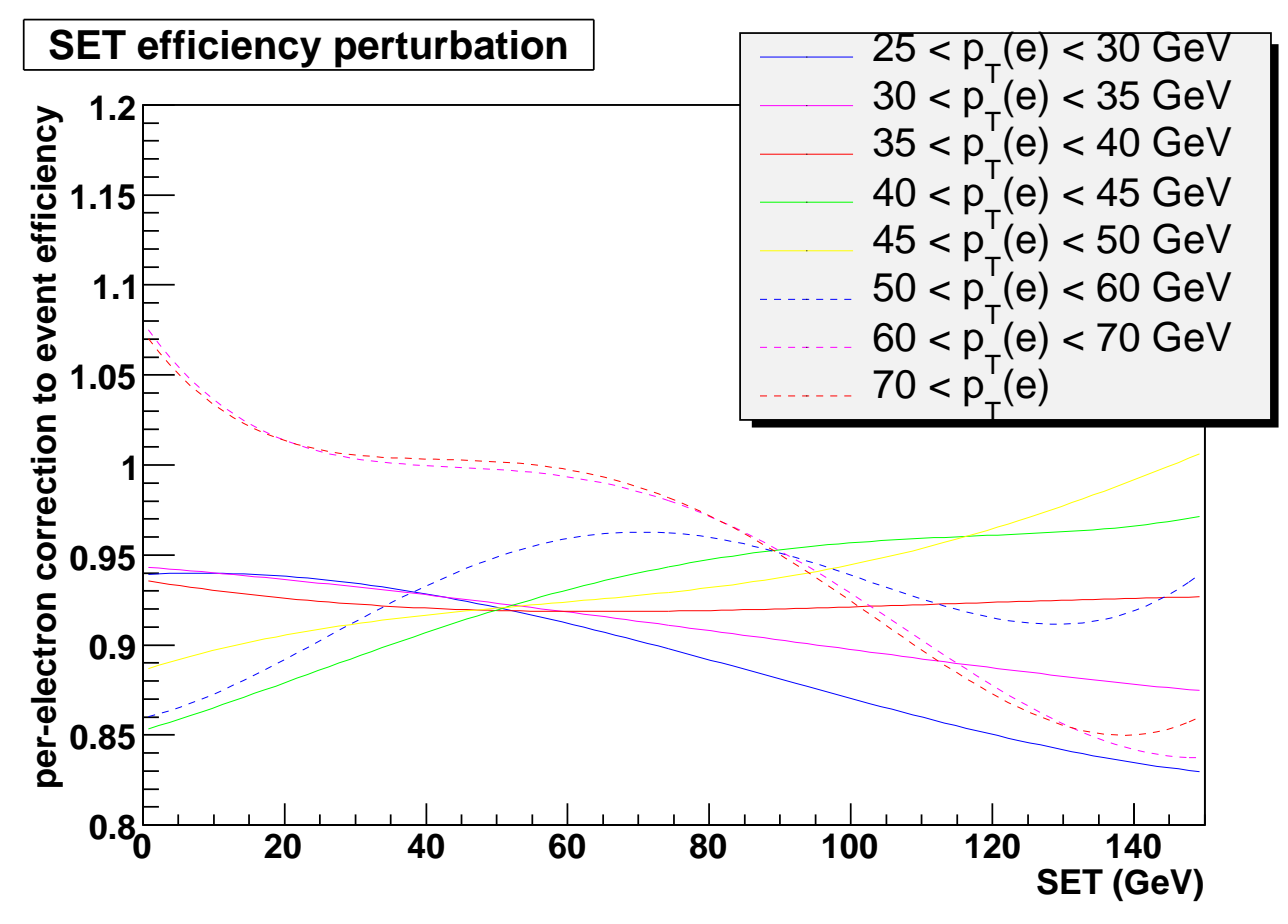

Figure 8.7: The $p_{\mathrm{T}}(e)$ based corrections on the overall SET efficiency, as a function of SET.

\subsection{Overall Tag-and-Probe Corrections}

Some of the strongest sources of $p_{\mathrm{T}}(e)$ dependence in our efficiency model are full $\mathrm{MC}$ derived, where we exploit truth information. As such, they cannot be individually tuned to data. We expect that the full Monte Carlo is realistic enough to tune these efficiencies, but we would still like to check them against data. We use the tag-and-probe method to generate $p_{\mathrm{T}}$ dependent efficiencies in both data and Monte Carlo. If the ratio of the fast MC and data efficiencies is flat, it means that the overall $p_{\mathrm{T}}$ dependence of our model is correct to within the statistical power of the data. If not, we could apply this ratio to the fast $\mathrm{MC}$ model, to fix the $p_{\mathrm{T}}$ dependence. Figure 8.8 and shows the fast MC comparisons with data for the HMx and track match efficiency. The ratio of the two efficiencies is consistent with a flat line. This is also true when we measures these efficiencies for bins of $\eta$ and SET. We are satisfied that our efficiency model accurately describes the data. Figures 8.8 and 8.9 show comparisons between the $p_{\mathrm{T}}$ dependence of the HMx and track match efficiency for full MC and data (left), and the ratio of the two efficiencies, fitted with a 
zero-order polynomial (right).
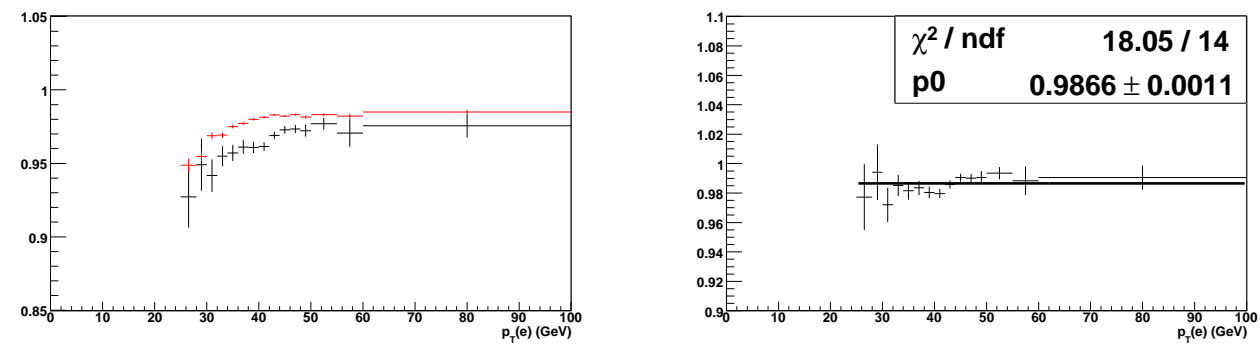

Figure 8.8: Left: $p_{T}$ dependence of HMx efficiency for CC electrons in data(black) and full MC(red); Right: Ratio between the black and red curve in the left plot.
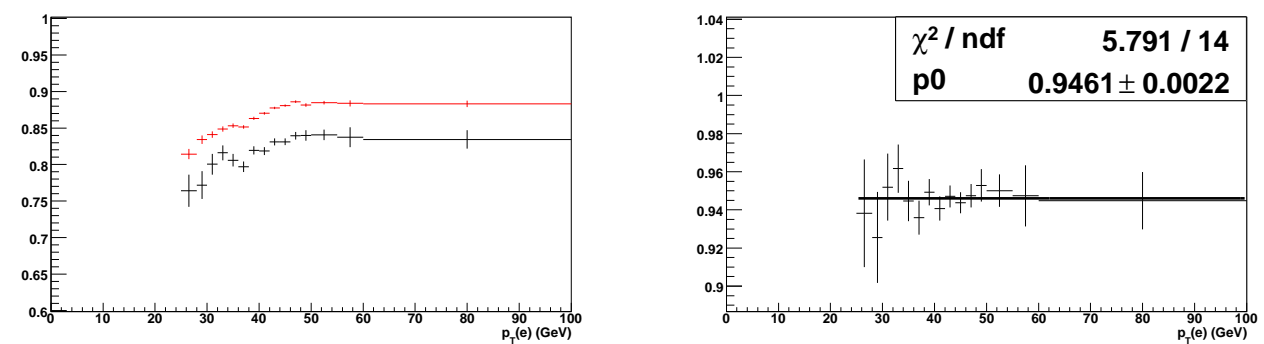

Figure 8.9: Left: $p_{T}$ dependence of track match efficiency for CC electrons in data(black) and full MC(red); Right: Ratio between the black and red curve in the left plot. 


\section{Chapter 9}

\section{Recoil Measurement and Simulation}

\subsection{Introduction}

Once we have an accurate description for measured electron energies in our $W \rightarrow e \nu$ model, it is necessary to describe the other key observable: the hadronic recoil.

Since the recoil is a multi-faceted object with many complex dependences, developing a parameterized model based upon first-principles is difficult and time-consuming. For this reason, we have developed a data-driven, heuristic approach to modeling the recoil. The QCD processes driving the formation of both the soft and hard hadronic content of an event are similar for $Z \rightarrow l l$ and $W \rightarrow l \nu$ events, a fact that is exploited in tuning any parameterized recoil model. The "Recoil Library Method" overlays simulated $W \rightarrow e \nu$ events with recoils extracted directly from $Z \rightarrow e e$ data, chosen to match the $\mathrm{W}$ kinematics as closely as possible. This approach has luminosity dependences and complex zero-suppression induced correlations built-in. It requires no a priori understanding of the hadronic energy content of $\mathrm{W}$ events, and has no adjustable parameters. This method is based on work done by D. P. Salzberg [1]. The details on our new implementation of the approach described in [86]

In this Chapter we will describe the Recoil Library Method, along the necessary Bayesian unfolding method, and we discuss its systematic uncertainties. We will also briefly describe the parameterized recoil model used in the Run II D0 W mass measurement, since we will use this model as a cross-check. The hadronic recoil plays a direct role in the calculation of two of the primary observables in the $\mathrm{W}$ mass measurement: the $M_{\mathrm{T}}$ and the $E_{T}$. It also figures indirectly in the electron $p_{\mathrm{T}}$ measurement through a kinematic requirement on our data the $p_{\mathrm{T}}(W)<15 \mathrm{GeV}$. Especially because the recoil model is one of the dominant systematics in the $W$ boson width measurement, it is important that we model it properly. 


\subsection{Recoil Library Method}

\subsubsection{Overview}

The recoil library is built from $Z \rightarrow e e$ events. Because the electron energy and angular resolutions are well measured, the measured $p_{T}^{Z}$ from the two electrons provides a good first approximation of the true $p_{T}^{Z}$. An unfolding procedure transforms the map between the measured $p_{T}^{Z}$ and the measured $u_{T}$ to a map between the true $p_{T}^{Z}$ and the measured $u_{T}$. The opening angle between the measured $p_{T}^{Z}$ and the measured $u_{T}$ is also unfolded to the opening angle between the true $p_{T}^{Z}$ and the measured $u_{T}$ during this procedure. A map between the true $p_{T}^{Z}$, the measured $u_{T}$, and the scalar $E_{T}(\mathrm{SET})$, which is the scalar sum of the transverse energies of all calorimeter cells outside the electron window, is also produced. This map is not used by the recoil model, but is needed by the electron efficiency model.

\subsubsection{Preparing the Recoil Library}

Before producing a binned recoil library, certain event-by-event corrections must be applied to the measured recoil system. We need to remove any biases in the measured recoil distribution due to the $Z$ selection requirements. Electron identification requirements, for example, preferentially reject events with high hadronic activity and thus poorer recoil resolution. Since $Z$ candidates contains two electrons while $W$ candidates only have one, the bias will not be the same. The $Z$ electrons also a higher average $p_{T}$ and a different $\eta$ distribution than $W$ electrons. To account for this, we remove the biases from the $Z$ selection, and then, when a $W$ event is made using the recoil library, the biases appropriate for a $W$ event are added, as described in Ref. [?]. In this section, we describe those corrections.

\subsubsection{Removing The Two Electrons from $Z$ Decays}

The recoil system for $Z \rightarrow e e$ events is defined as all of the energy in the calorimeter excluding the cells that belong to the two electrons from $Z$ decays. Since the recoil system will in general deposit energy in these cells, excluding them biases the component of the recoil in the 
electron directions. We correct this effect by adding back an approximation of the underlying energy.

This correction (denoted by $\Delta u_{\|}$) depends on $u_{\|}$(the projection of $\vec{u}_{T}$ along the electron transverse direction), instantaneous luminosity, and electron $\eta$, and is estimated using the momentum flow under equivalent cells separated in $\phi$ from the electron in $W \rightarrow e \nu$ events. In addition to correcting for the recoil energy under the electron cluster, we also correct for electron energy that leaks outside of the cluster. This energy leakage is subtracted from the recoil after the $\Delta u_{\|}$ is added back in. In Section 9.5 we estimate the uncertainty due to these corrections.

\subsubsection{Minimizing the Effects of FSR Photons}

The full MC simulation indicates that roughly $6 \%$ of the $Z \rightarrow e e$ events contain FSR photons with $E_{T}>400 \mathrm{MeV}$ that are far from the electrons so that the electron clustering algorithm does not merge them with the reconstructed electrons. These photons are thus incorrectly used in the measurement of $u_{T}$, instead of $p_{T}^{Z}$, resulting in a correlated bias. Since $Z \rightarrow e e$ events contain more FSR photons than $W \rightarrow e \nu$ events, the $Z$ recoil library will contain on average larger contributions from FSR photons.

Ideally, these FSR photons could be removed from the recoil file, and the effect could be separately modeled within the fast MC simulation. Since it is difficult to identify these FSR photons on an event-by-event basis, the effect is reduced by tightening the lower cut on the effective reconstructed di-electron invariant mass to $85 \mathrm{GeV}$, reducing the fraction of events with a high $p_{T}$ FSR photon by $25 \%$.

The effect of the remaining photons is small because, for low $p_{T} W$ boson, $M_{T} \approx 2 p_{T}(e)+u_{\|}$. Therefore, the photons will create a bias on the mass if they produce a bias in the component of $u_{T}$ parallel to the electron direction. While the overlaid recoil is rotated so that the direction of its corresponding $Z$ boson matches that of the simulated $W$ boson, the directions of the decay electrons from $Z$ and $W$ are largely uncorrelated and the bias is mostly canceled. In Section 9.5 the bias due to the FSR photons is measured. 


\subsubsection{Correcting for Electron Selection Efficiencies}

The selection criteria for $W$ and $Z$ events can introduce biases between the electron and the recoil system. Since the kinematic and geometric properties of $W$ candidates are not identical to those of $Z$ candidates, they have different biases.

The two components of the electron selection efficiency model that most strongly affect these biases are the SET efficiency and the $u_{\|}$efficiency. The SET efficiency describes the electron identification probability as a function of the overall activity in the detector. The $u_{\|}$efficiency describes the probability of electron identification as a function of $u_{\|}$. The probability decreases with increasing hadronic activity along the electron direction.

Since the recoil library is built from $Z \rightarrow e e$ events, we need to remove the biases introduced by the selection requirements on the two electrons. We correct for, or "invert" the $Z$ efficiencies by reweighting each event in the $Z$ recoil library by $1 / \epsilon_{u_{\|}} \times 1 / \epsilon_{S E T}$, where $\epsilon_{u_{\|}}$is the product of the efficiencies and $\epsilon_{S E T}$ is the product of the SET efficiencies for both electrons in each $Z$ event.

When $W$ events are produced from a fast MC using the recoil library, the map between the true $p_{T}^{Z}$, measured $u_{T}$, and SET is used to introduce the biases appropriate for $W$ 's from these efficiencies.

To simulate a $W$ boson event, a random recoil is chosen for the true $W$ boson $p_{T}$ from the recoil library, and a random SET is also chosen from the SET distribution corresponding to the true $W$ boson $p_{T}$ and the chosen recoil $u_{T}$. The $u_{\|}$efficiency and SET efficiency are then applied on the electron from $W$ boson decay.

\subsection{Bayseian Unfolding}

\subsubsection{Unfolding Method}

After the recoils have been corrected above, the transformation from measured $p_{T}^{Z}$ and measured $u_{T}$ to true $p_{T}^{Z}$ and measured $u_{T}$ can be done using a Bayesian unfolding technique. 


\subsubsection{Multidimensional Unfolding Using Bayes Theorem}

Unfolding is a mathematically challenging problem, since it involves the reversal of a random process. Because a given "true" state can fluctuate to many measured states or many different true states can fluctuate to the same measured state, we cannot unfold detector effects on an event-by-event basis. Rather, unfolding methods typically work with binned distributions.

For the recoil library method, we chose to use a Bayesian unfolding approach [87]. This approach suits our needs because it is intuitive, simple to implement, and easy to extend to the multidimensional case. The Bayesian technique uses conditional probabilities to determine the probability that a given measured state corresponds to a particular true state.

Consider a distribution of initial states $I_{i}\left(\right.$ true $\left.p_{T}^{Z}\right),\left\{i=1,2,3, \ldots, N_{I}\right\}$, given by $P\left(I_{i}\right)$ (the probability of events with initial state $I_{i}$ ) and a distribution of final states $F_{j}$ (measured $\left.p_{T}^{Z}\right),\left\{j=1,2,3, \ldots, N_{F}\right\}$ given by $P\left(F_{j}\right)$ (the probability of events with final state $F_{j}$ ). Given the measured distribution $P\left(F_{j}\right)$, and the probability for each initial state to fluctuate to each final state $P\left(F_{j} \mid I_{i}\right)$, we can determine the distribution of initial states $P\left(I_{i}\right)$ using

$$
P\left(I_{i}\right)=\sum_{j=1}^{N_{F}} P\left(F_{j}\right) P\left(I_{i} \mid F_{j}\right) .
$$

Using simulations, we can calculate $P\left(F_{j} \mid I_{i}\right)$, the likelihood of a true state fluctuating to a measured state. We calculate $P\left(I_{i} \mid F_{j}\right)$ from $P\left(F_{j} \mid I_{i}\right)$ using Bayes' theorem, which states:

$$
P(A \mid B)=\frac{P(B \mid A) P(A)}{P(B)}
$$

which for our particular example, with $N_{I}$ initial states and $N_{F}$ final states, Bayes' theorem gives us:

$$
P\left(I_{i} \mid F_{j}\right)=\frac{P\left(F_{j} \mid I_{i}\right) P\left(I_{i}\right)}{\sum_{k=1}^{N_{I}} P\left(F_{j} \mid I_{k}\right) P\left(I_{k}\right)}
$$

We can interpret this equation as follows: the probability that a given final state $F_{j}$ came from a particular initial state $I_{i}$ is proportional to the probability density of state $I_{i}$, multiplied by the probability that $I_{i}$ would fluctuate to $F_{j}$. The denominator normalizes the distribution.

Our Bayesian method requires us to make assumptions regarding the distribution of initial states, $P\left(I_{i}\right)$. Although we only use $P\left(I_{i}\right)$ to calculate the weights used to reweight the measured 
data, the quality of the unfolding could depend on our $P\left(I_{i}\right)$. To minimize this effect, the method is applied iteratively, starting with a guess distribution for $P_{0}$, and with each successive iteration using the previous iteration's unfolded distribution as the new input. As a cross-check the method is applied with several different initial guess distributions. The procedure for the iteration is:

1. Choose an initial input distribution for $P_{0}\left(I_{i}\right)$.

2. Using, $P_{0}\left(I_{i}\right)$ and $P\left(F_{j} \mid I_{i}\right)$, compute the weights $P\left(I_{i} \mid F_{j}\right)$, as derived using the Bayesian formula shown in Eqn 9.3.

3. Using these weights, recalculate the unfolded truth distribution $P_{1}\left(I_{i}\right)$ from the relationship $P_{1}\left(I_{i}\right)=\sum_{j=1}^{N_{F}} P_{0}\left(F_{j}\right) P\left(I_{i} \mid F_{j}\right)$ described in Eqn 9.1.

4. Repeat the above steps with $P_{1}\left(I_{i}\right)$ as the starting distribution.

5. Iterate until the unfolded $P\left(I_{i}\right)$ converges.

\subsubsection{Unfolding the Recoil Distribution}

The recoil vector is described by the coordinates $\left[u_{T}, \psi\right]$, where $u_{T}$ is the measured recoil transverse momentum, and $\psi$ is the angle between the measured recoil and the true boson direction in the transverse plane. These recoil vectors are stored in an array of 2-dimensional recoil histograms (binned in $u_{T}$ and $\psi$ ), each histogram corresponding to a discrete bin in true $p_{T}^{Z}$.

In the implementation of Eqn 9.3, the initial state $I$ is specified by $\left[\left(p_{T}^{Z}\right)_{i}^{t}, \psi_{j}, u_{T k}\right]$ and the final state $F$ is given by $\left[\left(p_{T}^{Z}\right)_{m}^{s}, \psi_{n}^{s}, u_{T k}\right]$, where $\left(p_{T}^{Z}\right)^{t}$ is the true $Z$ boson transverse momentum, $\left(p_{T}^{Z}\right)^{s}$ is the smeared $Z$ transverse momentum, and $\psi^{s}$ is the angle between the smeared $Z$ boson and the measured hadronic recoil.

We start with an initial guess distribution that is flat in $\left(p_{T}^{Z}\right)^{t}, \psi$ and $u_{T}$. We find that it takes fewer than 10 iterations for the unfolding method to attain convergence. The systematic error on the $W$ boson mass and width due to the unfolding procedure will be discussed further in Section 9.5. 
Figure 9.1 shows an example distribution of the probabilities that a $Z$ boson with a reconstructed $p_{T}^{Z}$ of $7 \mathrm{GeV}$ and a $3.5 \mathrm{GeV}$ recoil $u_{T}$ corresponds to different true $p_{T}^{Z}$ values. These probabilities are used to reweight the given recoil as we store it in the recoil histograms corresponding to the true $p_{T}^{Z}$ 's.

Figures 9.2-9.6 show various recoil observables plotted versus the true $p_{T}^{Z}$, obtained from the MC truth information of these MC samples compared with the same observables plotted versus the reconstructed $p_{T}^{Z}$, before and after the unfolding is applied. The unfolding corrects for average effects of $p_{T}^{Z}$ smearing on both the means and the RMS's of these recoil observables.

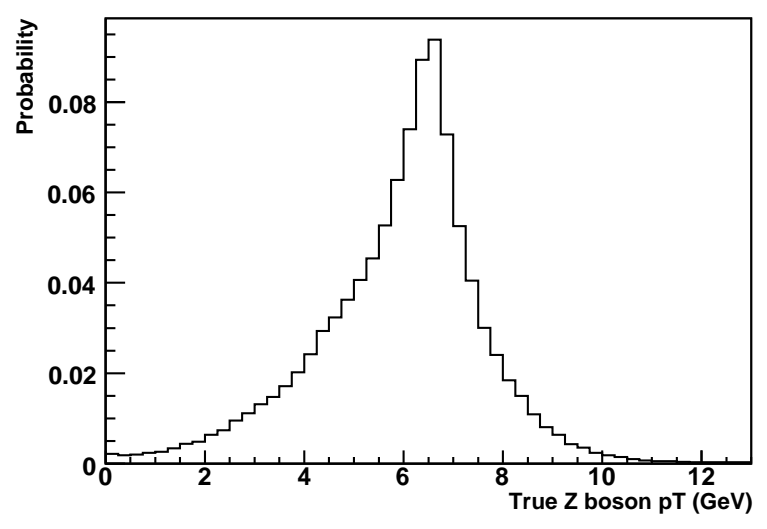

Figure 9.1: The distribution of the probabilities that a reconstructed $p_{T}^{Z}$ of $7 \mathrm{GeV}$ with corresponding $u_{T}$ of $3.5 \mathrm{GeV}$ came from various true $p_{T}^{Z}$ bins. 

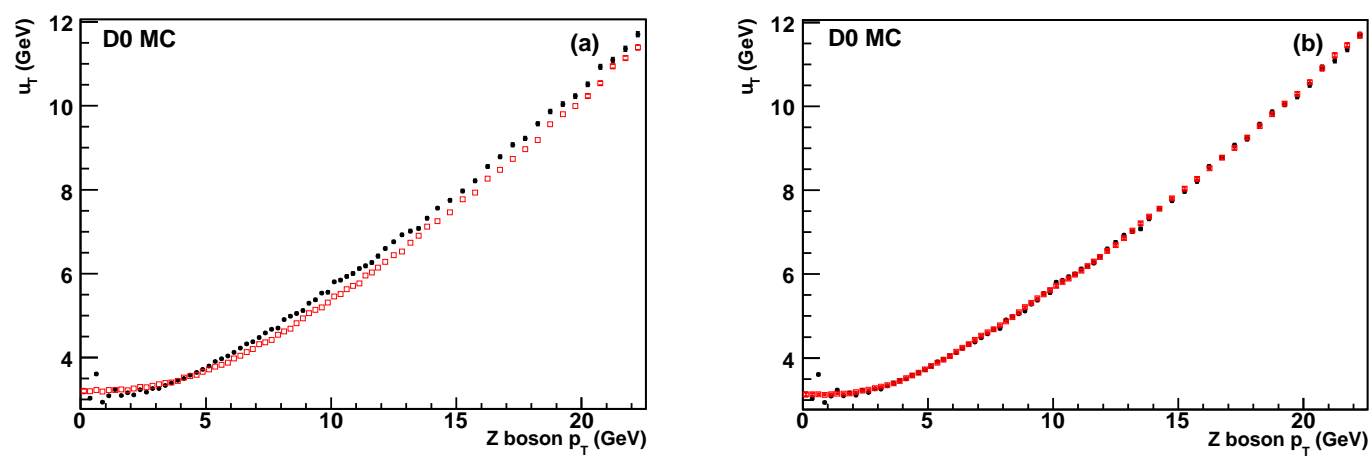

Figure 9.2: Mean recoil $u_{T}$ versus true $p_{T}^{Z}$ (black filled points) compared with mean recoil $u_{T}$ versus the estimate of the true $p_{T}^{Z}$ (red open boxes) when using (a) the two smeared electrons directly and (b) using the unfolded map.
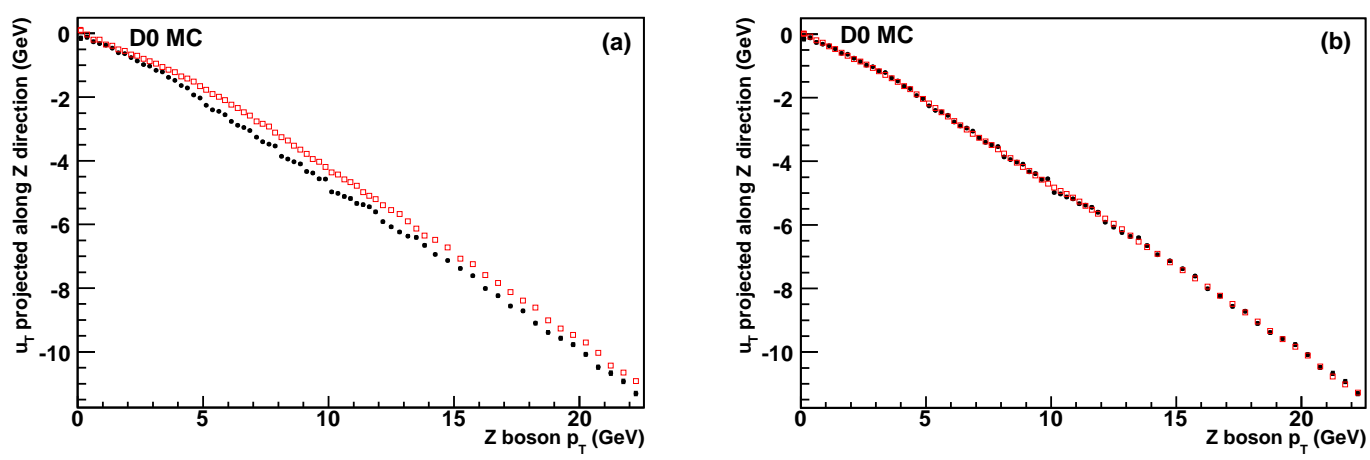

Figure 9.3: Mean projection of the recoil along the boson direction versus true $p_{T}^{Z}$ (black filled points) compared with mean projection of the recoil along the boson direction versus the estimate of the true $p_{T}^{Z}$ (red open boxes) when using (a) the two smeared electrons directly and (b) using the unfolded map. 

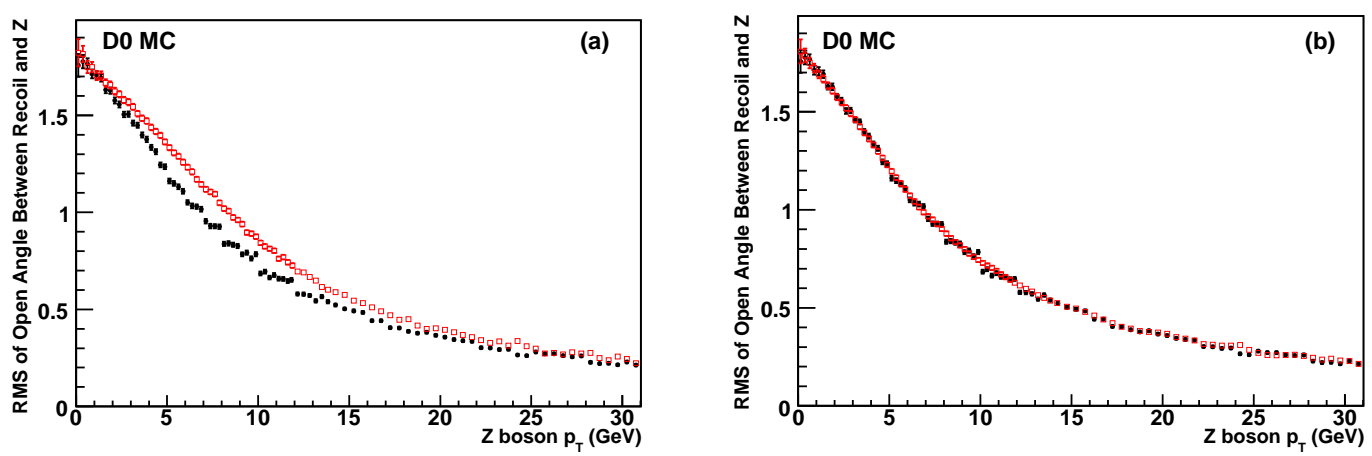

Figure 9.4: RMS of the opening angles between the recoil and the boson versus true $p_{T}^{Z}$ (black filled points) compared with RMS of the opening angle between the recoil and the boson versus the estimate of the true $p_{T}^{Z}$ (red open boxes) when using (a) the two smeared electrons directly and (b) using the unfolded map.
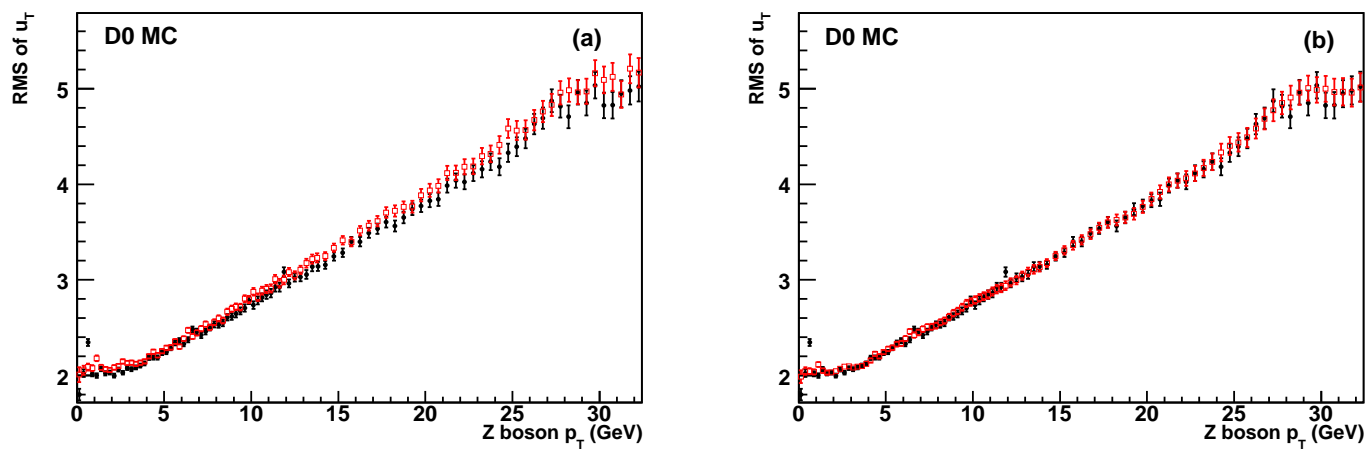

Figure 9.5: RMS of the recoil $u_{T}$ versus true $p_{T}^{Z}$ (black filled points) compared with RMS of the recoil $u_{T}$ versus the estimate of the true $p_{T}^{Z}$ (red open boxes) when using (a) the two smeared electrons directly and (b) using the unfolded map. 

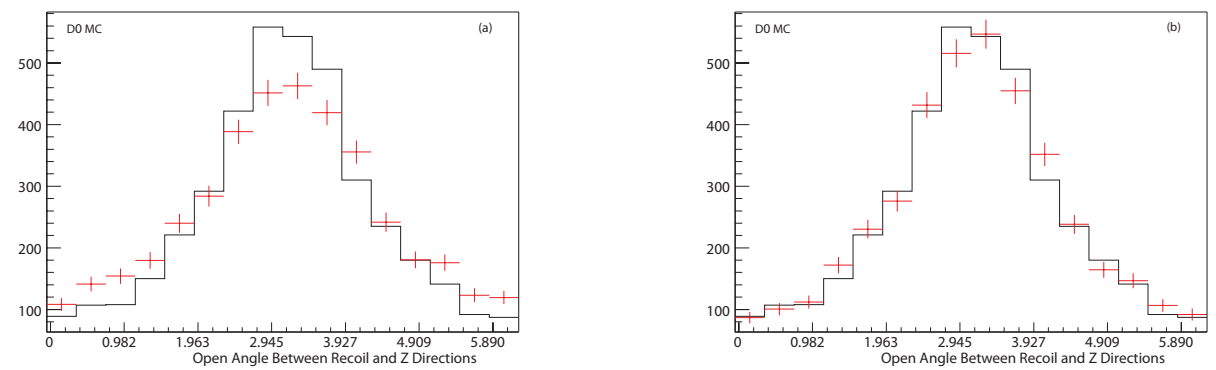

Figure 9.6: Angle between the measured recoil and the true $Z$ boson direction (solid line) and angle between the measured recoil and the measured $Z$ boson direction (points with error bars) when using (a) the two smeared electrons directly and (b) using the unfolded map for $Z$ events with a true $p_{T}^{Z}$ of 4.0 to $4.25 \mathrm{GeV}$. 


\subsection{A Brief Description of the Parameterized Recoil Model}

The Parameterized Recoil Method models the detector response to the hard recoil using a 2-dimensional parameterization that smears the true hard recoil in both direction and magnitude. The underlying event, as well as pileup and additional interactions are modeled using a combination of minbias and zerobias libraries taken from data with the same luminosity profile. These effects are then combined with the hard recoil and data-tuned corrections are applied to account on average for correlations between the "hard" and "soft" recoil due to detector effects such as zero suppression. The correction parameters are then tuned to either collider data or full MC, depending on the study.

\subsection{Biases and Uncertainties Particular to the Recoil Library Method}

In order to do very high statistics tests of the efficacy of the recoil library, we study the mass and width values obtained by comparing fast MC $W$ boson samples created using the parameterized recoil model and templates generated from simulated $W$ boson event samples incorporating $Z$ recoil libraries. The $Z$ recoil libraries were generated from $Z \rightarrow e e$ events created with the parameterized recoil model. By varying parameters in the simulation used to generate the $W$ boson samples while leaving the templates unchanged, we measure any biases and the statistical and systematic uncertainties on the extracted numbers.

\subsubsection{Fast MC Closure and Limited Statistical Power of the $Z$ Recoil Sample}

Because we use $Z$ recoils to model $W \rightarrow e \nu$ events, we face statistical limitations. In $1 \mathrm{fb}^{-1}$ of data, after the selection cuts, we expect roughly $18,000 Z \rightarrow e e$ events with both electrons in the central calorimeter, whereas in the same data we expect roughly 500,000 W $\rightarrow e \nu$ events with the electron in the central calorimeter. Templates made using the $Z$ recoil method will need to contain millions of events. In these events we chose recoil vectors from the same set of 18,000 $Z \rightarrow e e$ events. Our method is thus limited by the size of the $Z$ recoil sample and any statistical fluctuations it contains. If we are to rely on this method as an input to a precision measurement, we 
need to determine the extent to which the statistical limitations of the $Z \rightarrow e e$ sample propagate to an uncertainty on the measured $W$ boson mass and width.

We assess the statistical uncertainties of the recoil method using an ensemble of 100 fast MC simulations (toy models) resembling the statistical situation we expect in real data. We generate $W$ and $Z$ samples corresponding to $1 \mathrm{fb}^{-1}$ of data using the parameterized recoil method. For each set of $W$ and $Z$ samples, we use the $Z$ boson events to create a recoil library and then use the library to create templates for the recoil in the simulated $W$ boson events. These templates are then used to extract the $W$ mass and width. The statistical power is measured using the spread of extracted masses and widths from these toy models.

Figure 9.7 shows the measured $W$ boson masses from $M_{T}$ distributions from 100 toy models using the $M_{T}$ distribution. Figure 9.8 shows the corresponding distributions for the $W$ boson width. The mean fit value is in good agreement with the true value, showing that the recoil library can accurately model the parameterized recoil method. We test that the recoil library can model the full $\mathrm{MC}$ in a later section.

The statistical uncertainty on the mass measurement due to the recoil library method is $5 \mathrm{MeV}$ from the $M_{T}$ spectrum, $8 \mathrm{MeV}$ for the $p_{T}(e)$ spectrum, $17 \mathrm{MeV}$ for the $E_{T}$ spectrum. These agree with the statistical uncertainties on the parameterized recoil model, which are found to be $6 \mathrm{MeV}$ for the $M_{T}$ fit, $7 \mathrm{MeV}$ for the $p_{T}(e)$ fit and $19 \mathrm{MeV}$ for the $E_{T}$ fit. The statistical uncertainty on the width measurement due to the recoil library method is $40 \mathrm{MeV}$ using the $M_{T}$ spectrum, and agrees with the statistical uncertainty of $42 \mathrm{MeV}$ using the parameterized recoil model.

Even though one might naively expect that the additional information contained in the functional form used in the parameterized method would give it increased statistical power. The results listed above show that we do not lose substantial statistical power by directly using the limited $Z$ data for the recoil model. We use a simplified detector model of $W$ and $Z$ boson events with and without recoil energy resolution effects added, and then compare the " $\eta$-imbalance" distribution (defined as the difference between magnitudes of the boson $p_{T}$ and the projection 
of the recoil $u_{T}$ along the boson direction) for the parameterized and library methods. Due to the similar transverse momentum profiles of the $W$ and $Z$ bosons, we find that the means of the $\eta$-imbalance distribution agree with each other within statistical uncertainty. We also find that without recoil energy resolution effects, there is a clear but small $(\mathcal{O}(100) \mathrm{MeV})$ increase in the RMS of the $\eta$-imbalance distribution for the recoil library method, but with the resolution effects added, the RMS of the $\eta$-imbalance distribution increases to over $2 \mathrm{GeV}$ and masks any difference stemming from the difference of the parameterized and library methods.

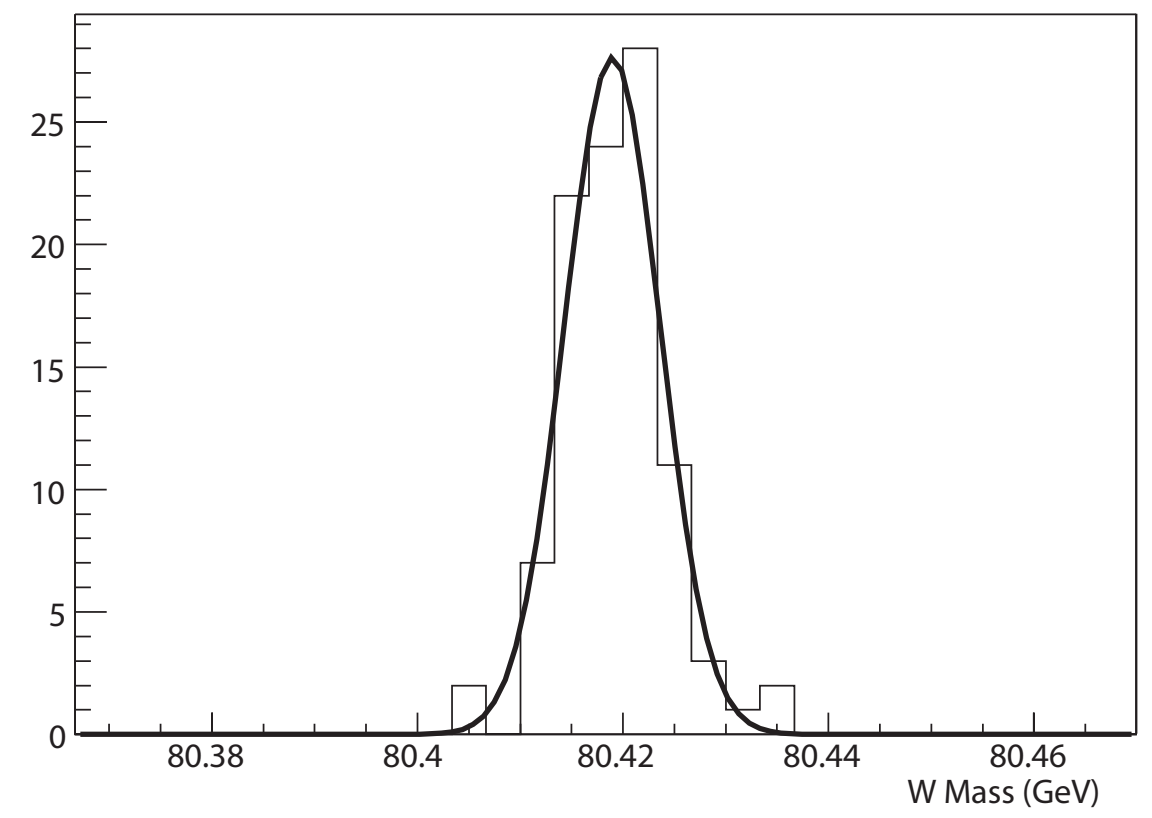

Figure 9.7: Mean $W$ boson mass measured in 100 ensemble tests for each template generated from a recoil file. The black line is a fit using a Gaussian function. All toy models were generated with an input $W$ boson mass of $80.419 \mathrm{GeV}$ and the fitted gaussian function has a mean value of $80.420 \pm 0.001 \mathrm{GeV}$ and the fitted gaussian width is $0.005 \pm 0.001 \mathrm{GeV}$.

\subsubsection{Systematic Effects}

We mentioned in Section 9.2 that several effects could potentially bias the recoil library method. These include unmerged FSR photons, acceptance differences between $Z$ and $W$ events, 


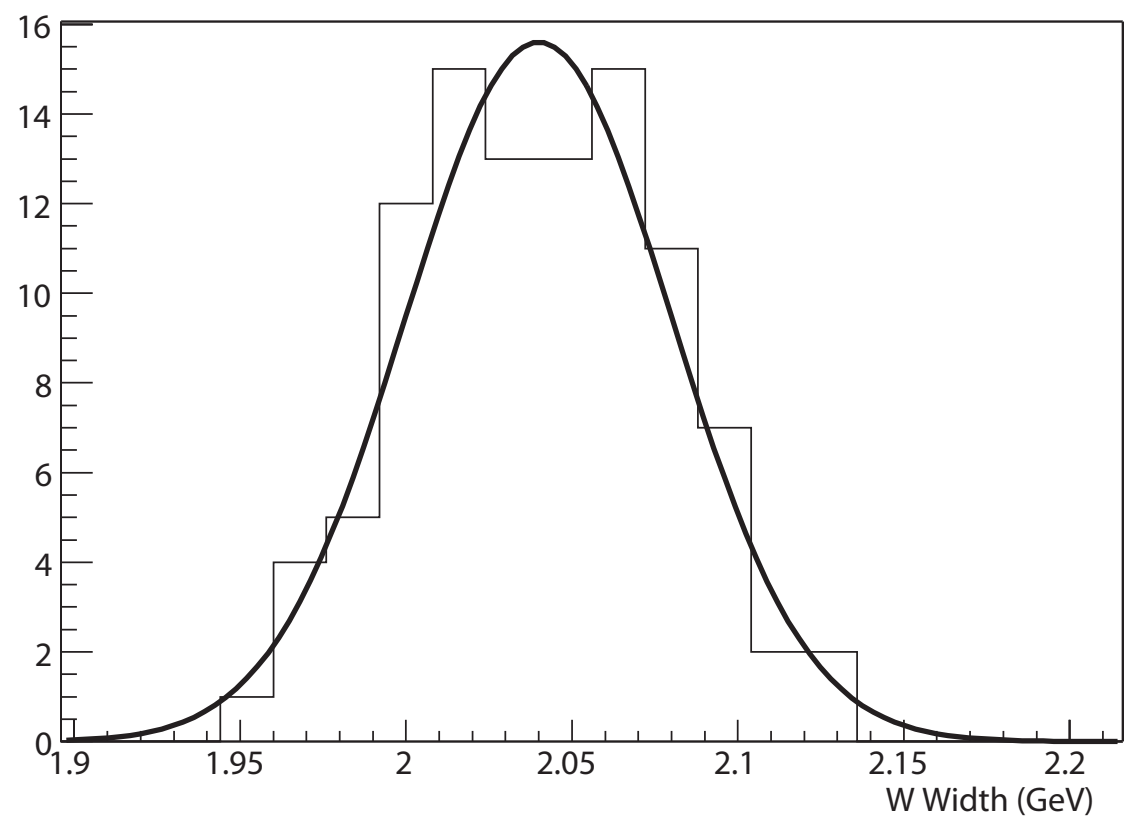

Figure 9.8: Mean $W$ boson width measured in 100 ensemble tests for each template generated from a recoil file. The black line is a fit using a Gaussian function. All toy models were generated with an input $W$ boson width of $2.039 \mathrm{GeV}$ and the fitted gaussian function has a mean value of $2.040 \pm 0.001 \mathrm{GeV}$ and the fitted gaussian width is $0.040 \pm 0.003 \mathrm{GeV}$. 
the unfolding process, residual efficiency-related correlations between the electron and the recoil system, and underlying energy corrections beneath the electron window. The closure tests using fast MC described in Section 9.5.1 show the overall bias from this method to be smaller than the statistical power of the tests. Nonetheless, we want to make sure that this small final bias is not due to the cancellation of larger individual biases and therefore examine each effect independently.

\subsubsection{Unmerged FSR Photons}

We measure the residual bias due to FSR photons by fitting two sets of toy data models, one made from an unfolded, high statistics recoil file with all FSR photons included, and one made from an equivalent recoil file with no FSR photons. We find that the mass shift between these two samples is $-1 \mathrm{MeV}$ for the $M_{T}$ fit, $-2 \mathrm{MeV}$ for the $p_{\mathrm{T}}(e)$ fit, $2 \mathrm{MeV}$ for the $E_{T}$ fit, and the width shift is less than $1 \mathrm{MeV}$.

\subsubsection{Differences in Geometric Acceptance}

For $W$ events, we only require the electron to be in the central calorimeter, while for $Z$ events used to create the library, we require both electrons to be in the central calorimeter. To test the bias due to this effect, we generate two recoil files. For one recoil file we restrict both electrons to the central region of the detector, as we would in data. For the other recoil file, we restrict only one electron and allow the other electron to be anywhere in the detector, like a neutrino. We make templates from the two independent recoil files and find that the difference in both measured mass and measured width is consistent with zero within the statistical uncertainty of this high-statistics study from the fast MC's.

\subsubsection{Efficiency Related Biases}

When we generate the truth-level and unfolded recoil files, we reweight the events by the reciprocals of the $u_{\|}$and SET efficiencies, as described in Section 9.2.5. To check if this approach introduces any biases, we perform fast MC closure measurements using templates made from truth-

level recoil files. We perform this test three times. Once, we only apply the $u_{\|}$efficiency to the toy 
model, the toy-recoil file, and the template set. Likewise, we only correct for the $u_{\|}$efficiency when making the recoil library. In the second test, we only apply the SET efficiency, and in the final test we apply both efficiencies. We conservatively estimate the uncertainty due to the efficiency model as being the maximum bias in the fitted mass or width over all three tests. Table 9.1 shows the biases for all three variations. The final uncertainty attributed to the efficiency corrections on the $W$ boson mass is $7 \mathrm{MeV}$ for the $M_{T}$ fit, $7 \mathrm{MeV}$ for the $p_{T}(e)$ fit, $8 \mathrm{MeV}$ for the $E_{T}$ fit. The uncertainty on the $W$ boson width is found to be $7 \mathrm{MeV}$.

Table 9.1: Difference between fitted values and input values for three variations of the truth-level, fast MC closure test: only $u_{\|}$efficiency applied, only SET efficiency applied, and both efficiencies applied.

\begin{tabular}{|l|c|c|c|c|}
\hline Test & $\Delta M_{W}(\mathrm{MeV})\left(M_{T}\right)$ & $\Delta M_{W}(\mathrm{MeV})\left(p_{T}(e)\right)$ & $\Delta M_{W}(\mathrm{MeV})\left(E_{T}\right)$ & $\Delta \Gamma_{W}(\mathrm{MeV})$ \\
\hline$u_{\|}$efficiency only & -4 & -7 & -1 & +5 \\
SET efficiency only & -7 & -2 & -8 & +4 \\
both efficiencies & 0 & +2 & -1 & +7 \\
\hline
\end{tabular}

\subsubsection{Uncertainty in $\Delta u_{\|}$}

In Section 9.2.2 we observed that by removing the electrons from the $Z \rightarrow e e$ recoil file, we also remove some of the recoil energy that underlies the electron cones. We correct for this effect by adding back the average energy, $\Delta u_{\|}$, expected beneath the electrons. We then subtract the electron energy that leaks outside of the electron cone that is wrongly attributed to the recoil energy.

We assess the systematic uncertainty due to these corrections as follows: We make $Z$ recoil files for each of three cases: (1) No energy corrections; (2) A constant energy correction for underlying hadronic energy beneath the electron cone and constant correction for the electron energy leakage; (3) The parameterized energy correction for underlying hadronic energy beneath 
the electron cone and constant correction for the electron energy leakage.

We then generate three set of templates from each of these three recoil files, and measure the shift in fitted $W$ boson mass between these three template sets. The $W$ boson mass shifts by $2 \mathrm{MeV}$ for the $M_{T}$ fit, $4 \mathrm{MeV} p_{T}(\mathrm{e})$ fit, $1 \mathrm{MeV}$ for the $E_{T}$ fit, and $7 \mathrm{MeV}$ for the width. We conservatively assign the magnitude of these maximum shifts as the error on this method due to the $\Delta u_{\|}$correction.

\subsubsection{Uncertainties Due to Implementation of Unfolding}

The specific choices made in implementing the unfolding could introduce biases to the final measurement. Our results may depend on our choice of initial distributions in $\left(p_{T}^{Z}\right)^{t}, \psi$ and $u_{T}$. They could also depend on the number of iterations of the unfolding procedure we apply to the recoil library.

It was found that starting with flat initial distributions in $\left(p_{T}^{Z}\right)^{t}, \psi$ and $u_{T}, 10$ iterations was sufficient to attain convergence. We generate unfolded recoil files using two different initial distributions and using 8, 10, and 12 iterations of the unfolding method. We find that the change in measured mass and width extracted from $M_{T}, p_{T}(e)$, and $E_{T}$ fits is negligible. In addition to unfolding the recoil file using a flat initial distribution for the recoil spectrum, we also tried several smoothly varying sinusoidal guess distributions, and again find that the variation in the final unfolded recoil file is negligible.

\subsubsection{Total Systematic Uncertainties Due to the Recoil System Simulation}

Tables 9.2 shows the estimated systematic uncertainties due to the recoil system simulation for $1 \mathrm{fb}^{-1}$ of MC data. The overall systematic uncertainties are found to be $9 \mathrm{MeV}$ using the $M_{T}$ fit, $12 \mathrm{MeV}$ using the $p_{T}(e)$ fit and $19 \mathrm{MeV}$ using the $E_{T}$ fit for the $W$ mass, and $41 \mathrm{MeV}$ using the $M_{T}$ fit for the $W$ width. 
Table 9.2: Estimated systematic uncertainties on the $W$ boson mass and width from the recoil library method, for $1 \mathrm{fb}^{-1}$ of $Z$ data.

\begin{tabular}{|l|c|c|c|c|}
\hline Source & $\sigma\left(M_{W}\right) M_{T}$ & $\sigma\left(M_{W}\right) p_{\mathrm{T}}(e)$ & $\sigma\left(M_{W}\right) E_{T}$ & $\sigma\left(\Gamma_{W}\right) M_{T}$ \\
$(\mathrm{MeV})$ & $(\mathrm{MeV})$ & $(\mathrm{MeV})$ & 4 \\
\hline Recoil statistics & 5 & 8 & 2 & 1 \\
FSR photons & 1 & 2 & 8 & 7 \\
Efficiency related bias & 7 & 7 & 1 & 7 \\
$\Delta u_{\|}$ & 2 & 4 & 1 & 1 \\
\hline Unfolding & 1 & 1 & 19 & 41 \\
\hline Systematic total & 9 & 12 & & 17 \\
\hline
\end{tabular}




\section{Chapter 10}

\section{Backgrounds}

In this Chapter we discuss backgrounds to our $W$ boson measurement.

\subsection{Fake Rate}

The fake rate gives the probability that a QCD jet will have a fake track match and be misidentified as an electron. The data sample we use to measure the fake rate, called "EM + jet" consists of events with two back-to-back jets where one jet passes the jet ID requirements and the other passes the EMID requirements. The fake rate $f_{Q C D}$ is the ratio of the number of "EM+jet" events with a matching track to the total number of events in the sample.

\subsection{Backgrounds}

Some fraction of collider events fake $W \rightarrow e \nu$ events, well enough to pass the strict selection criteria imposed. These events can alter the shape of the relevant fit observables and must be modeled in order to accurately extract the mass and width. The three dominant backgrounds in $W \rightarrow e \nu$ events are the $Z \rightarrow e e$ background, $W \rightarrow \tau \nu \rightarrow e \nu \nu \nu$, and QCD dijet events where one jet fakes an electron. In this section we will look at these backgrounds. Further information can be found in [89].

\subsection{1 $Z \rightarrow e e$ background}

On occasion one of the two electrons in a leptonic $Z$ boson decay will be lost in an uninstrumented region like a $\phi$-crack or the Inner Cryostat Region (ICR). In these cases, the $Z \rightarrow e e$ event will look very much like a leptonic $W$ boson decay. We estimate these backgrounds directly from data, because our GEANT detector model is not accurately modeling the ICR detector. We take data that pass our standard $W \rightarrow e \nu$ selection, and require an isolated high $p_{\mathrm{T}}$ track, back-to-back 
and oppositely charged with respect to the identified electron. We also require that the track have a high $\eta_{\text {det }}$ corresponding to the ICR, and that the invariant mass of the track and EM object be between 70 and $110 \mathrm{GeV}$. Our measured background fraction is found to be $(0.80 \pm 0.01) \%$.

\subsection{2 $W \rightarrow \tau \nu$ Background}

The $\tau$ lepton in a $W \rightarrow e \nu$ decay can decay into an electron and two neutrinos, thus mimicking the signature of a $W \rightarrow e \nu$ decay. Fortunately this background is largely suppressed by kinematics and the low branching fraction for the $\tau \rightarrow e \nu \nu$ decay. We study this background in full MC and find it to be small $(1.6 \pm 0.02) \%$.

\subsubsection{QCD Background}

The QCD background is determined for each bin of each observable distribution using the "Matrix Method". The Matrix Method starts with a "tight" and "loose" sample of $W$ boson events. For the tight sample, we use our standard $W \rightarrow e \nu$ data set, and we drop the track match requirement for the loose sample. We know both the track efficiency $\epsilon_{t r k}$ and the QCD fake-rate $f_{Q C D}$. The number of $W \rightarrow e \nu$ events in the two samples provides us with two equations to solve for the two unknowns, $N_{W}$ and $N_{Q C D}$, namely:

$$
N=N_{W}+N_{Q C D}
$$

and

$$
N_{t r k}=\epsilon_{t r k} N_{W}+f_{Q C D} N_{Q C D}
$$

where $N$ is the total number of events measured in the "loose" sample, $N_{W}$ is the number of signal events, $N_{Q C D}$ is the number of background events, and $N_{t r k}$ is the number of observed events in the "tight" sample with the track selection cut applied. This calculation can be performed in bins of the three fit observables to derive the shape of the background, in addition to the total number of events. 
10.2.4 Final Background Distributions

Figures 10.1, 10.2, and 10.3 show the shapes of the final background distributions for the $M_{\mathrm{T}}, E_{T}$, and $p_{\mathrm{T}}(e)$ fits, respectively.

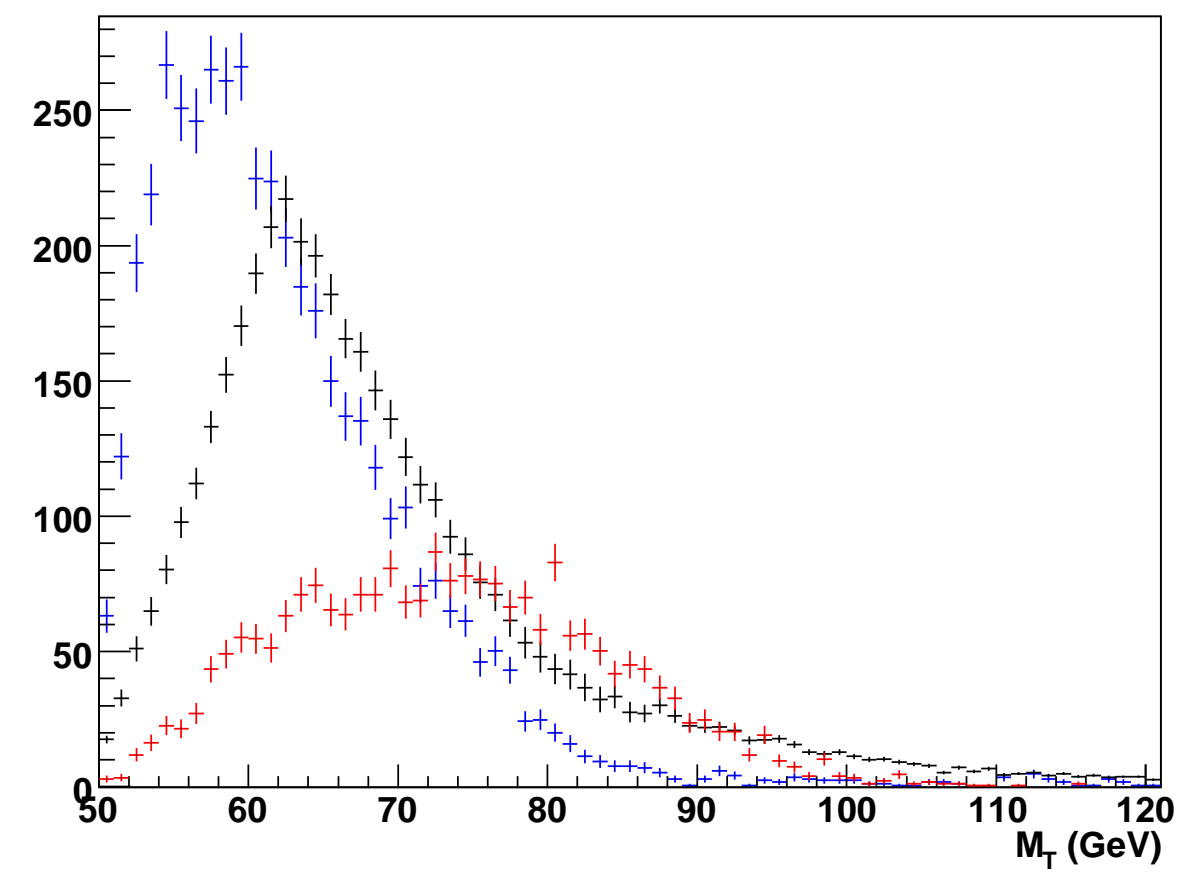

Figure 10.1: The three background shapes for the $M_{\mathrm{T}}$ distribution: QCD (black), $Z \rightarrow e e($ red), and $W \rightarrow \tau \nu$ (blue) 


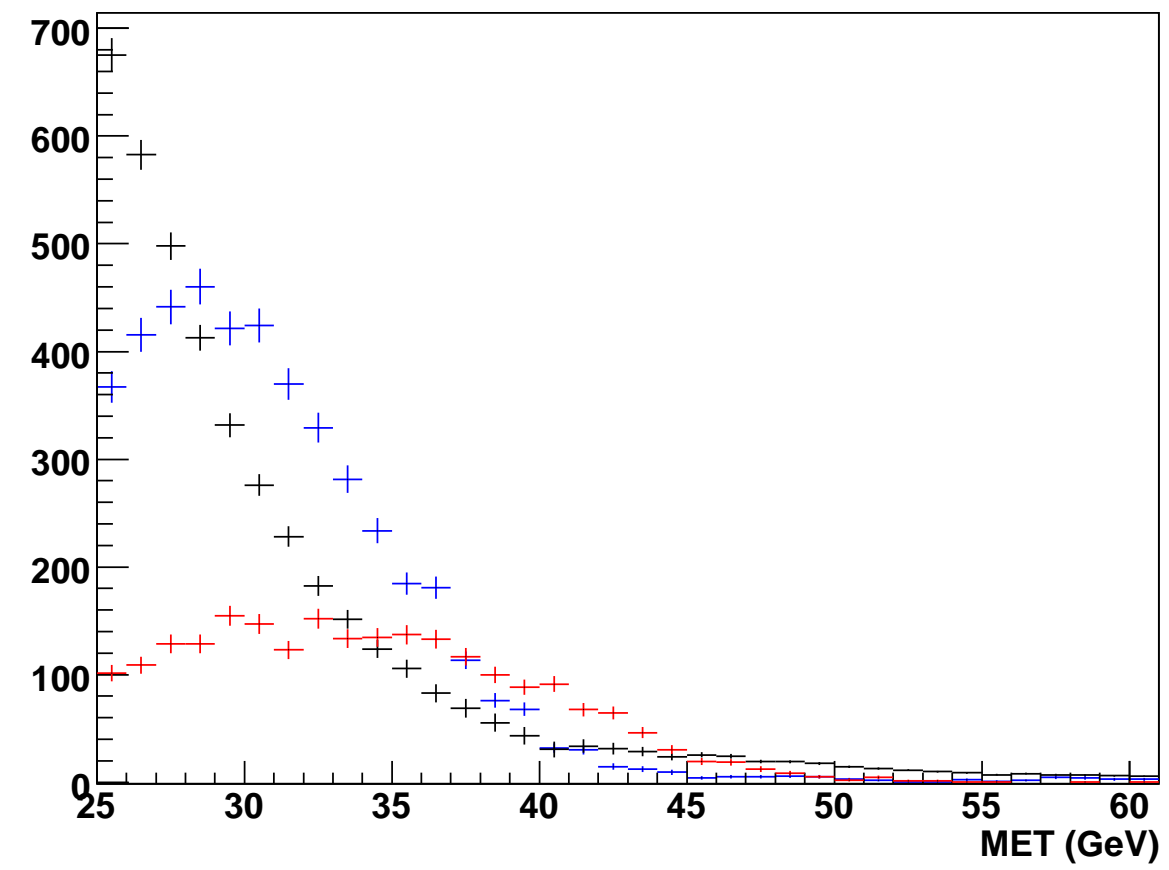

Figure 10.2: The three background shapes for the $E_{T}$ distribution: QCD (black), $Z \rightarrow e e$ (red), and $W \rightarrow \tau \nu$ (blue) 


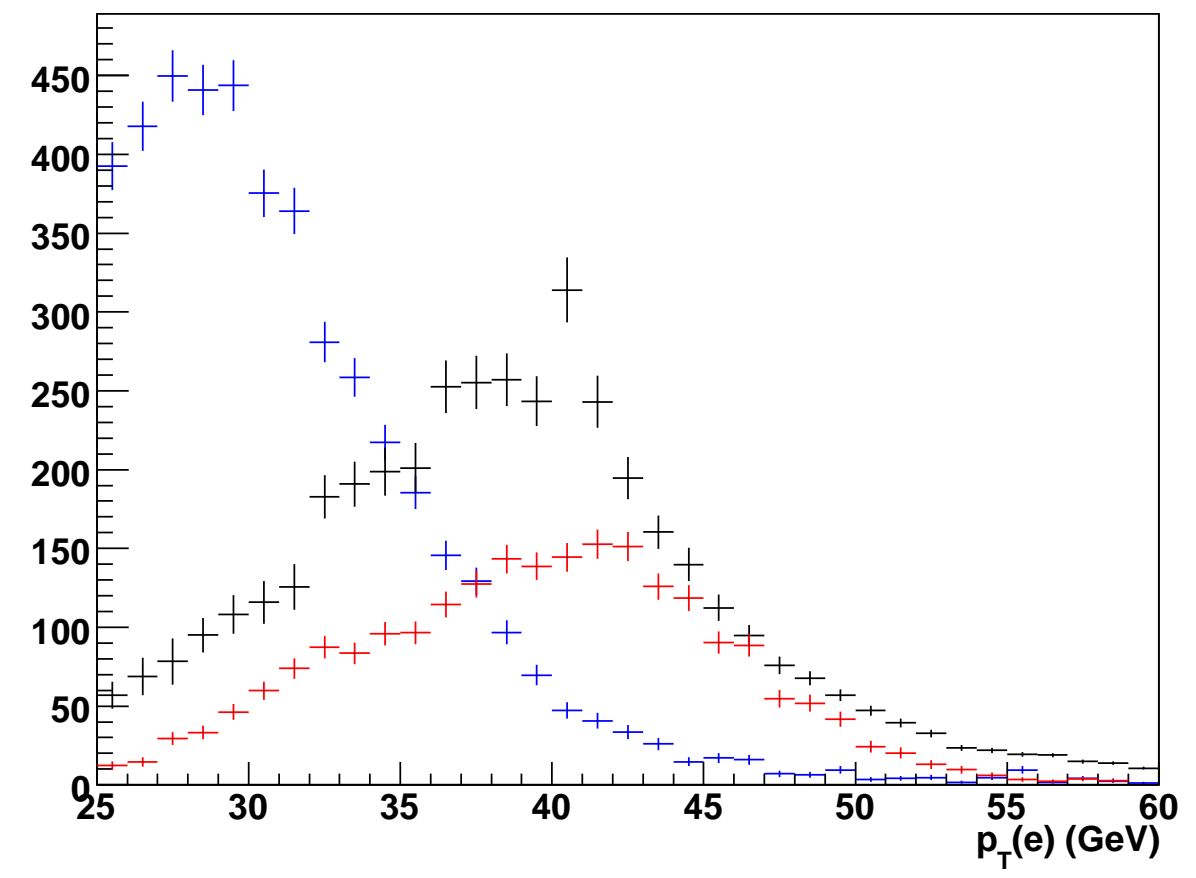

Figure 10.3: The three background shapes for the $p_{\mathrm{T}}(e)$ distribution: QCD (black), $Z \rightarrow e e$ (red), and $W \rightarrow \tau \nu$ (blue) 


\section{Chapter 11}

\section{Full Monte Carlo Closure Tests}

In addition to our parameterized simulations, we test the effectiveness of the recoil library method on a full detector simulation designed to resemble data as closely as possible. We apply the $Z$ recoil library method, using the full Monte Carlo $Z \rightarrow e e$ sample, to generate $W \rightarrow e \nu$ templates. The full Monte Carlo $W$ boson mass and width are extracted from fits using these templates, and compared with the true input values. After cuts, our full detector Monte Carlo $Z$ boson sample has the equivalent of roughly $6 \mathrm{fb}^{-1}$ in integrated luminosity, and our W sample has roughly $2.5 \mathrm{fb}^{-1}$. This closure test is similar to the full MC study described in [90].

Before determining the full MC mass and width, we test the accuracy of our model by comparing various full MC distributions to our fast $\mathrm{MC}$ model at the truth value of the $\mathrm{W}$ mass. Figure 11.1 shows comparisons between $W \rightarrow e \nu$ full Monte Carlo and fast Monte Carlo using the Recoil Library Method for the $M_{T}, p_{\mathrm{T}}(e)$, and $p_{\mathrm{T}}(\nu)$ distributions. Figure 11.2 shows comparison plots for the $\mathrm{Z}$ full and fast Monte Carlo. Additional control plots also give good agreement between fast MC and full MC.

Table 13.3 shows the final full Monte Carlo mass and width measurements, performed using the recoil library method. Approximated systematic uncertainties on the electron model, dominated by the uncertainty on the energy scale, are $15 \mathrm{MeV}$ for $M_{\mathrm{T}}$ and MET mass measurement, $12 \mathrm{MeV}$ for $p_{\mathrm{T}}(e)$ mass measurement, and $15 \mathrm{MeV}$ for the width. Systematic uncertainties on the hadronic model are taken from Chapter 9 of this paper. The results of our full Monte Carlo measurements agree with the full MC input mass of $80.450 \mathrm{GeV}$ and input width of $2.071 \mathrm{GeV}$ to within the uncertainties. These results are also consistent with the full MC measurements using the parameterized recoil model, given in Table 11.2. 

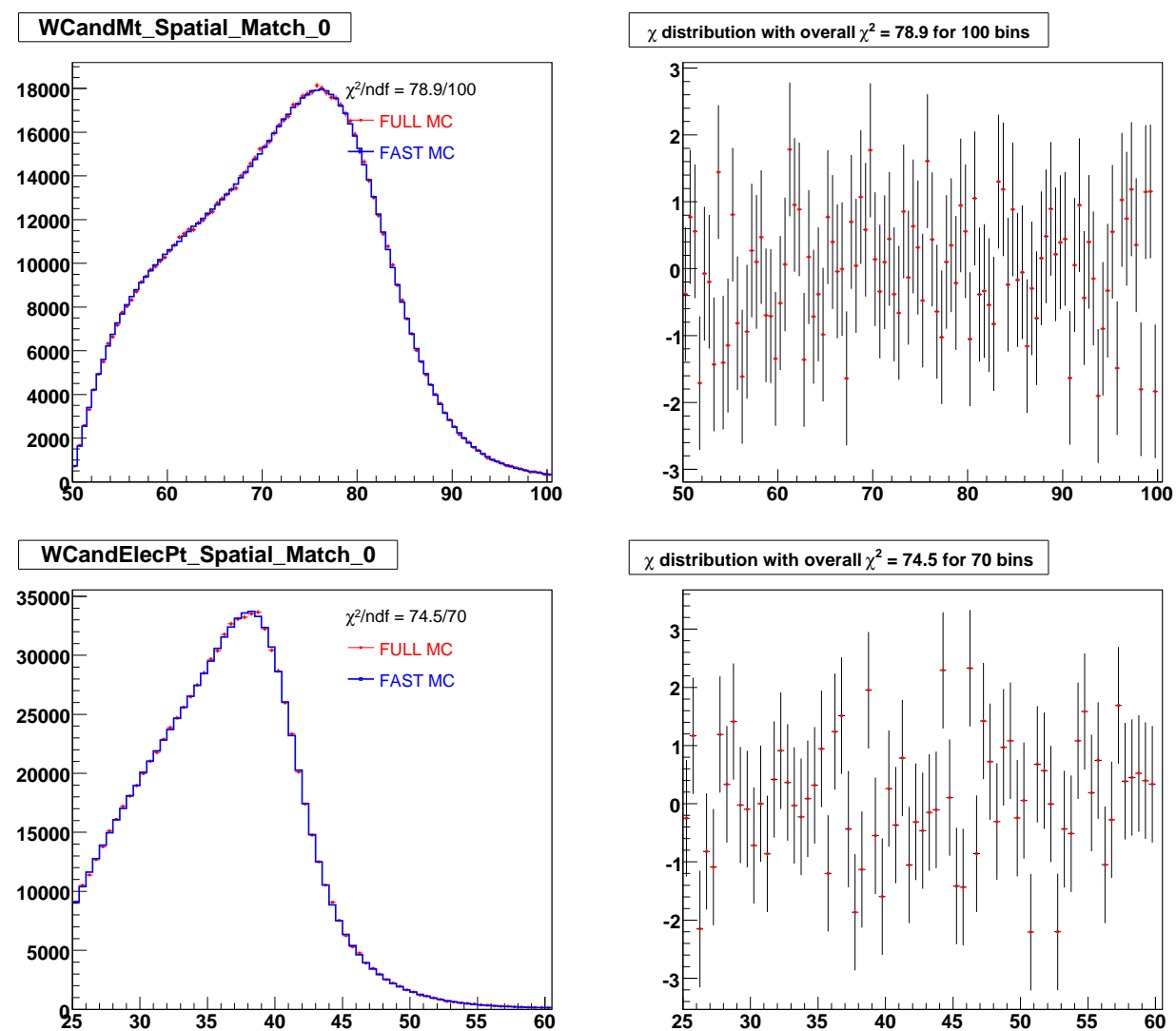

WCandMet_Spatial_Match_0
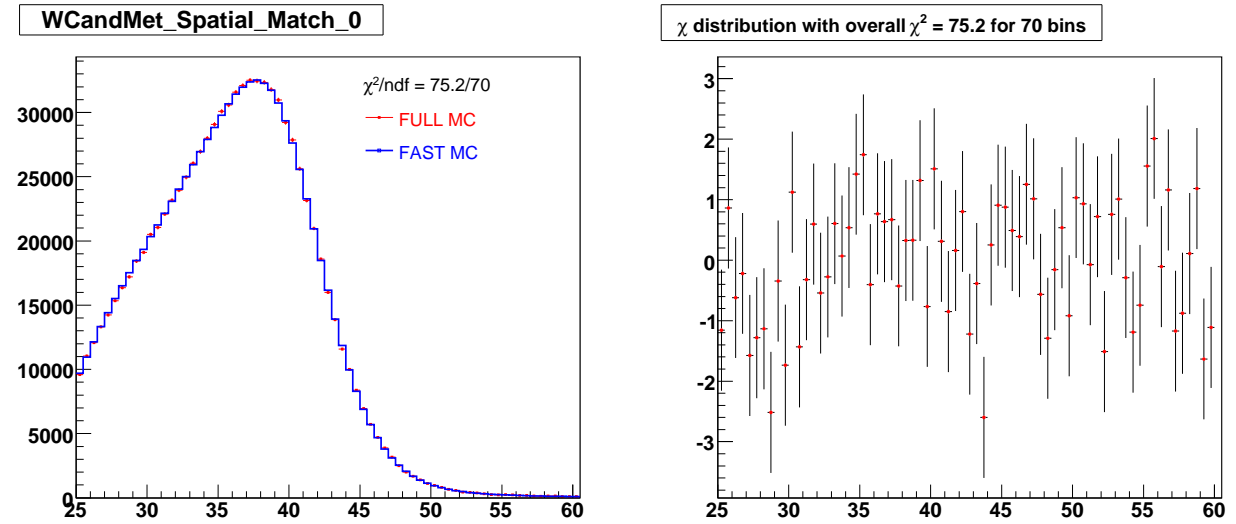

Figure 11.1: Comparison plots between full MC (points) and fast MC (lines) for the $\mathrm{W} M_{\mathrm{T}}$ distribution (top), W electron $p_{\mathrm{T}}$ distribution (middle), and MET distribution (bottom). Corresponding $\chi$ plots are shown on the right side. 
ZCandMass_CCCC_Trks

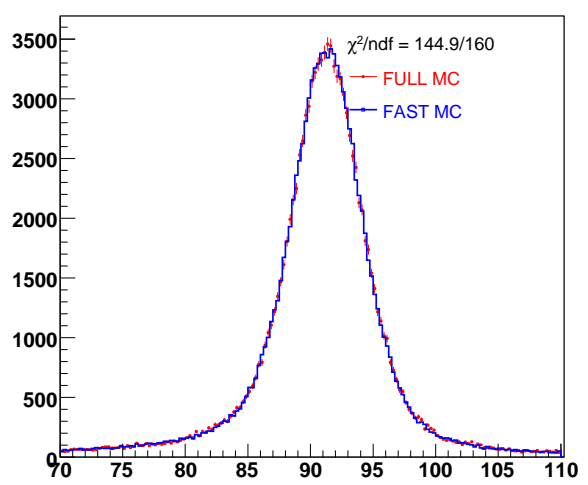

ZCandRecoilPt_0

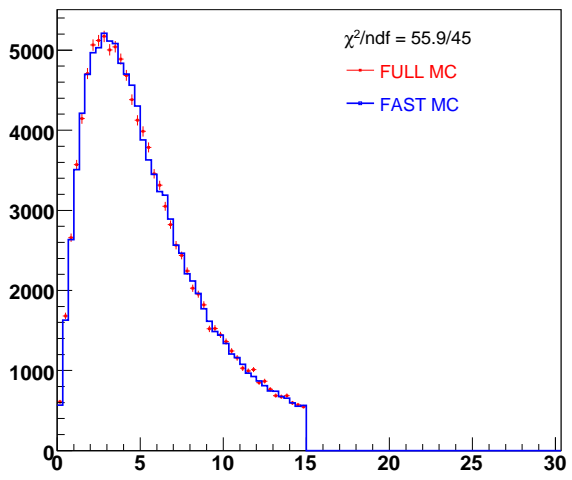

ZCandPt_0

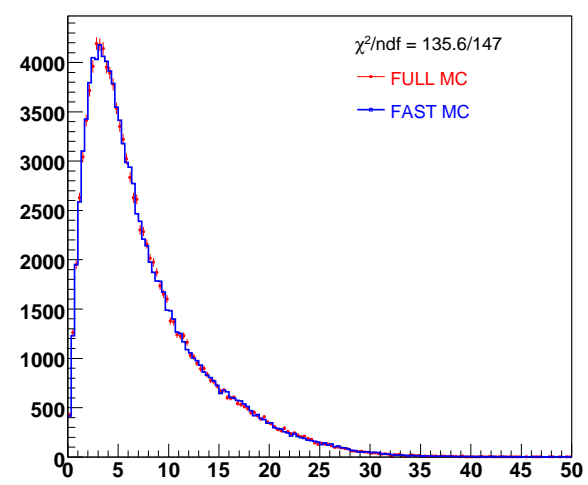

ZCandElecPt_0

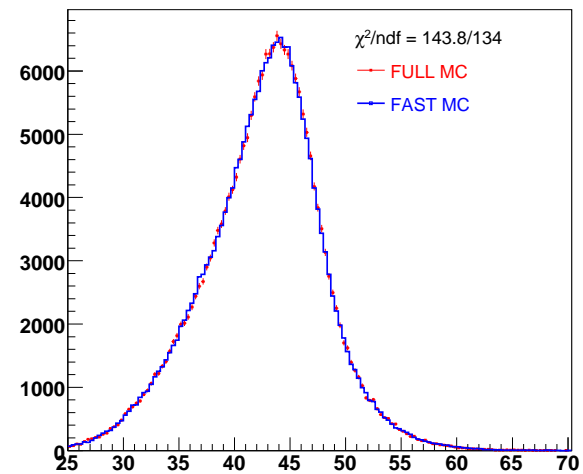

Figure 11.2: Comparison plots between full MC (points) and fast MC (lines). Top left: Z mass distribution. Top right: $\mathrm{Z} p_{\mathrm{T}}$ distribution, reconstructed from the di-electron $p_{\mathrm{T}}$. Lower left: $\mathrm{Z}$ Recoil $p_{\mathrm{T}}$ distribution. Lower right: $\mathrm{Z}$ electron $p_{\mathrm{T}}$ distribution.

\begin{tabular}{|l|l|}
\hline Variable & Fitted Mass $[\mathrm{GeV}]$ \\
\hline$M_{\mathrm{T}}$ & $80.456 \pm 0.015($ stat $) \pm 0.015$ (elec syst) \pm 0.008 (recoil syst) \\
$p_{\mathrm{T}}(e)$ & $80.455 \pm 0.019$ (stat) \pm 0.012 (elec syst) \pm 0.009 (recoil syst) \\
$M E T$ & $80.450 \pm 0.019$ (stat) \pm 0.015 (elec syst) \pm 0.011 (recoil syst) \\
\hline & Fitted Width $[\mathrm{GeV}]$ \\
\hline$M_{\mathrm{T}}$ & $2.066 \pm 0.027$ (stat) \pm 0.015 (elec syst) \pm 0.019 (recoil syst) \\
\hline
\end{tabular}

Table 11.1: Final result of the full Monte Carlo closure fits for the $\mathrm{W}$ mass and width, using the recoil library method and the equivalent of $2 f^{-1}$ of $\mathrm{W}$ data and $6 f b^{-1} \mathrm{Z}$ recoil data. The input $\mathrm{W}$ mass value is $80.450 \mathrm{GeV}$, and the width value is $2.071 \mathrm{GeV}$. 


\begin{tabular}{|l|l|}
\hline Variable & Fitted Mass $[\mathrm{GeV}]$ \\
\hline$M_{\mathrm{T}}$ & $80.441 \pm 0.015$ (stat) \pm 0.015 (elec syst) \pm 0.002 (recoil syst) \\
$p_{\mathrm{T}}(e)$ & $80.441 \pm 0.019$ (stat) \pm 0.012 (elec syst) \pm 0.003 (recoil syst) \\
$M E T$ & $80.429 \pm 0.019$ (stat) \pm 0.015 (elec syst) \pm 0.007 (recoil syst) \\
\hline & Fitted Width $[\mathrm{GeV}]$ \\
\hline$M_{\mathrm{T}}$ & $2.065 \pm 0.027$ (stat) \pm 0.015 (elec syst) \pm 0.016 (recoil syst) \\
\hline
\end{tabular}

Table 11.2: Final result of the full Monte Carlo closure fits for the $\mathrm{W}$ mass and width, using the parameterized recoil model and the equivalent of $2 \mathrm{fb}^{-1}$ of $\mathrm{W}$ data and $6 f b^{-1} \mathrm{Z}$ recoil data. The input $\mathrm{W}$ mass value is $80.450 \mathrm{GeV}$, and the width value is $2.071 \mathrm{GeV}$. 


\section{Chapter 12}

\section{Systematic Uncertainties}

In this chapter we present the systematic uncertainties connected with the $M_{W}$ and $\Gamma_{W}$ measurements. Pseudo-experiments are produced from fast Monte Carlo, where a given parameter is varied by $\pm 1 \sigma$ and $\pm 2 \sigma$, with all other parameters fixed at their default values. These pseudoexperiments are are fit with fast MC templates generated with all of the parameters set to their respective defaults. It is assumed that the variation of the $W$ boson mass and width is roughly linear for these small perturbations about the default values. For a given observable we then have the formula:

$$
\sigma_{O b s}^{2}=\left(\frac{\partial O b s}{\partial X}\right)^{2} \sigma_{X}^{2}
$$

where Obs is the observable in question $\left(M_{W}\right.$ or $\left.\Gamma_{W}\right), X$ is the parameter in question, and $\frac{\partial O b s}{\partial X}$ is the slope of the uncertainty due to $X$.

\subsection{Theoretical Uncertainties}

Several uncertainties in our boson production and decay models contribute to the final uncertainty on the $W$ boson mass and width measurements.

\subsubsection{PDF Uncertainties}

One important theoretical input into the theoretical boson production model used in this analysis is the set of parton distribution functions (PDF) that describe the distribution of fractional momenta carried by the quarks and gluons in the $p \bar{p}$ pair that produces the $W$. These PDF sets are experimentally derived and limited precision. The precision of the PDF model must be propagated to the $W$ boson mass and width fits, to determine its impact on these measurements. We use the CTEQ6.1 PDF set, produced by the CTEQ collaboration, is in our RESBOs generator. These 
sets are described by an orthogonalized set of twenty independent parameters. We can very these parameters and measure the variation of $M_{W}$ and $\Gamma_{W}$ to determine their respective errors. In addition to the nominal PDF set, we have twenty pairs of 40 PDF sets, each pair corresponding to a $\pm 90 \%$ confidence level variation on a particular parameter with all other parameters fixed at their default values. The mass and width shifts are measured for each pair of PDF sets to provide the variation of the $W$ boson mass for variations of each parameter at $90 \%$ confidence level. These individuals are combined according to the formula"

$$
\sigma_{M_{W}}=\frac{1}{1.6} \frac{1}{2} \sqrt{\sum_{i=1}^{N}\left(M_{i}^{(+)}-M_{i}^{(-)}\right)^{2}}
$$

where $i$ corresponds to a particular parameter, $M_{i}^{(+)}$and $M_{i}^{(-)}$represent the measured mass (or width) for positive and negative variations of the parameter at $90 \%$ confidence, and the factor of 1.6 scales the $90 \%$ confidence to a one- $\sigma$ variation.

\subsubsection{Boson $p_{\mathrm{T}}$ Model}

The theoretical model describing the boson $p_{\mathrm{T}}$ spectrum is found to be most sensitive to the $g_{2}$ parameter of the BLNY parameterization of the non-perturbative region of the spectrum. This $g_{2}$ value, also experimentally measured, has a finite precision that impacts the resulting spectrum and the fast MC templates. The fast MC model describe in this dissertation use the global fit value of $0.68 \pm 0.02$. To assess the uncertainty on the $W$ boson mass and width, toy data are produced for \pm 0.02 variations on $g_{2}$ and fit with default templates to obtain the error derivative on $M_{W}$ and $\Gamma_{W}$ due to the $p_{\mathrm{T}}$ model.

\subsubsection{QED Model}

The QED uncertainty in our model corresponds to several effects involving final and initial state photon radiation. First, RESBOS, neglects higher-order processes, such as ISR-FSR interference and $\mathrm{W}$ and $\mathrm{Z}$ self-energy box diagrams. In addition, the uncertainty depends on our electron-photon merging model (described in Chapter 7). Lastly, we might be sensitive to the 
$400 \mathrm{MeV}$ lower cut on FSR photons. Preliminary tests using WGRAD and ZGRAD showed that higher order processes were negligible. For the uncertainties due to FSR merging and the photon $p_{\mathrm{T}}$ cut we set conservative upper limits by varying the cone size and $p_{\mathrm{T}}$ cuts by large amounts and propagating those variations to the $W$ boson mass and width measurements. The systematic uncertainty from the QED model cited in this dissertation is the sum of those two upper limits in quadrature.

\subsubsection{W Mass}

In the $W$ boson mass measurement, the uncertainty due to the assumed width is negligible. The fit for $\Gamma_{W}$, however, does depend on the assumed value of $M_{W}$. We determine this systematic using toy pseudo-experiments generated at various values for the $W$ boson mass. We fit variations in $\Gamma_{W}$ versus $M_{W}$ using a straight-line approximation for $\partial \Gamma_{W} / \partial M_{W}$. We multiply this derivative by $\sigma_{M W}(25 \mathrm{MeV})$ and find the systematic to be small but non-negligible.

\subsection{Experimental Uncertainties}

\subsubsection{Electron Energy Scale}

The dominant uncertainty in the $W$ boson mass measurement, and one of the larger uncertainties in the width measurement is the uncertainty due to the limited precision of the electron energy scale and offset derived from the $Z \rightarrow e e$ resonance. These two parameters are highly negatively correlated, and as a consequence we cannot merely propagate the uncertainties of these two parameters independently, since the uncertainties largely cancel. Rather, we combine the errors, in a correlated fashion according to the formula:

$$
\sigma_{\text {tot }}=\sqrt{\sigma_{\text {scale }}^{2}+\sigma_{\text {offset }}^{2}+\rho \sigma_{\text {scale }} \sigma_{\text {offset }}}
$$

where $\rho$ is the correlation coefficient between the energy scale and offset. 


\subsubsection{Electron Energy Resolution}

The electron energy resolution at the energies of $W$ and $Z$ boson decay electrons is dominated by the sampling and constant terms in our resolution model, described in Chapter 7. The uncertainty on the sampling resolution can be determined by propagating a $1-\sigma$ variation on the uncertainty of the missing material in the full MC detector model. The constant term is highly correlated with the sampling term, since it essentially measures the residual resolution effects beyond the sampling and noise terms. We vary the sampling term and remeasure the constant term. We propagate these correlated variations through our $W \rightarrow e \nu$ model and determine the uncertainty on the mass and width.

\subsubsection{Non-Linearity}

The electron E-loss corrections used to linearize the electron response of the calorimeter are tuned to our special full MC sample with missing material reintroduced. The uncertainty on that missing material could impact the linearity of the energy response. To test this systematic effect, we vary the missing material by its uncertainty and, leaving the energy scale and offset fixed, remeasure the $W$ boson mass and width. To within the precision of our detector understanding, we find that the E-loss corrections and linear electron energy scale provide an accurate detector description. The upper limit this measurement placed on the non-linearity is negligible.

\subsubsection{Hadronic Model}

The uncertainty due to the Recoil Library Method are estimated in Chapter 9 of this thesis. While not large for the $W$ boson mass, it is the dominant uncertainty in the width measurement.

\subsubsection{Efficiencies}

The uncertainty due to our efficiency model is dominated by the $u_{\|}$and SET efficiencies, which introduce the strongest dependence on kinematic variables.

The $u_{\|}$efficiency uncertainty has two parameters which we vary independently by one sigma 
and propagate to the $W$ boson mass and width. While the $W$ boson mass is sensitive to these parameters, especially over the $p_{\mathrm{T}}(e)$ and $E_{T}$ fits, the effect on the width is less than $1 \mathrm{MeV}$.

For the overall uncertainty on the $p_{\mathrm{T}}$, SET, and $\eta$ dependence of the efficiency, we look at ratios between the full MC to which we tune our fast $\mathrm{MC}$ and the data. We plot the ratio of the efficiencies in two 2D histograms, one histogram of SET versus $p_{\mathrm{T}}(e)$ and one histogram of $\eta$ versus $p_{\mathrm{T}}(e)$. We fit them with a plane, noting the values, uncertainties, and correlations on the slopes. The $\chi^{2}$ shows that the ratio of the efficiencies are consistent with a fit to a plane with slopes $<10^{-4}$ We find that the slopes in the $\eta$ and SET directions are almost entirely uncorrelated with the slope in the $p_{\mathrm{T}}$ direction, so we can propagate the uncertainties on these slopes independently. We apply 1-sigma variations on the slope of the efficiency ratio to reweight sets of toy data, and extract the $W$ boson mass and width from these toys. Figure 12.1 shows a plot of fits to these ratios. It is observed that variations in the slope in the SET and $\eta$ directions have a less than 1 $\mathrm{MeV}$ effect on the mass and width. The effects of variation in the overall $p_{\mathrm{T}}$ dependence are not negligible. We add this in quadrature with the estimated uncertainty on the $u_{\|}$efficiency and cite this as the conservative uncertainty due to the efficiency model.

\subsubsection{Backgrounds}

The binned background shapes used in the $W$ boson fits, come with statistical error bars. We generate variations on the background shapes by independently varying the contents of each bin according to gaussians with central values set to the nominal bin value and RMS values equal to the 1-sigma error bars. The nominal background shape is applied to a fast MC sample. A template set is produced to fit the fast MC sample. We apply thousands of statistical variations on the backgrounds to the same template set and refit for the mass and width.

\subsection{Summary of Systematic Uncertainties}

Tables 12.1 and 12.2 show summaries of the systematic uncertainties for the Run IIa $W$ boson mass and width fits using the Recoil Library Method. The uncertainties are comparable 


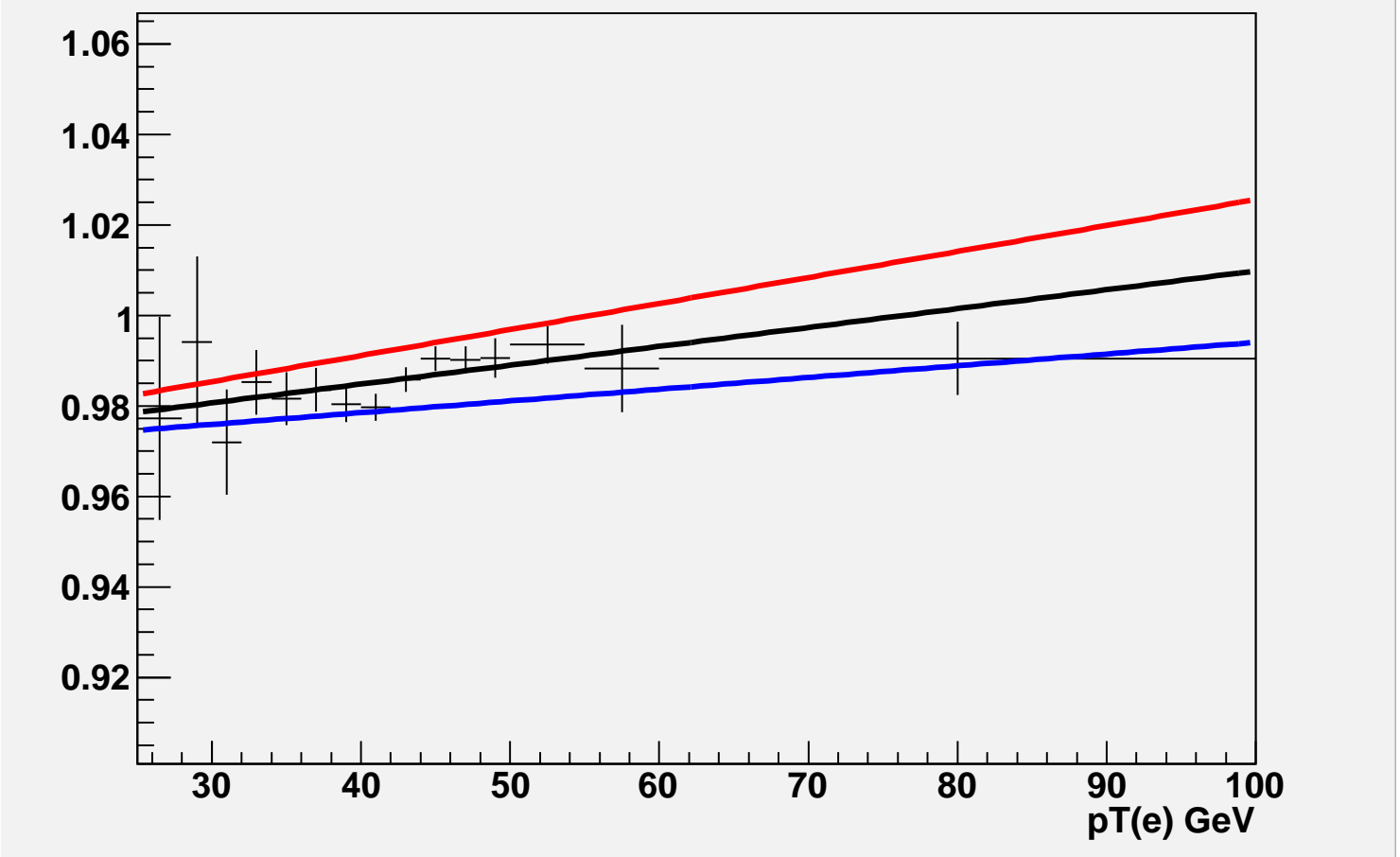

Figure 12.1: Ratio of the $p_{\mathrm{T}}$ dependence of the HMx efficiency between data and full MC (points), fit with the nominal fit function (black line) and with plus (red) and minus (blue) 1 sigma on the $p_{\mathrm{T}}$ dependent slope. 


\begin{tabular}{|l|c|c|c|}
\hline Source & $\sigma\left(M_{W}\right) \mathrm{MeV} p_{T}(e)$ & $\sigma\left(M_{W}\right) \mathrm{MeV} M_{T}$ & $\sigma\left(M_{W}\right) \mathrm{MeV} p_{T}(\nu)$ \\
\hline \hline Experimental & & & \\
\hline EM Scale & 34 & 34 & 34 \\
EM Resolution & 2 & 2 & 3 \\
Recoil Model & 9 & 12 & 19 \\
Efficiencies & 5 & 5 & 5 \\
Backgrounds & 37 & 35 & 41 \\
\hline Experimental Total & 11 & 9 & 14 \\
\hline W Model & 7 & 7 & 9 \\
\hline PDF & 5 & 2 & 17 \\
\hline QED & 14 & 38 & 43 \\
Boson $p_{T}$ & 39 & & 2 \\
\hline W model Total & & & \\
\hline Total & 5 & & \\
\hline
\end{tabular}

Table 12.1: Experimental and theoretical systematic uncertainties on the $\mathrm{W}$ boson mass measured with $1 \mathrm{fb}^{-1}$ in the $W \rightarrow e \nu$ channel.

to those using the Parameterized Recoil Model, and sufficiently low to guarantee a competitive 1 $\mathrm{fb}^{-1}$ measurement. 


\begin{tabular}{|c|c|c|c|}
\hline Fit Range & $\sigma\left(\Gamma_{W}\right) \mathrm{MeV}[90,200]$ & $\sigma\left(\Gamma_{W}\right) \mathrm{MeV}[100,200]$ & $\sigma\left(\Gamma_{W}\right) \mathrm{MeV}[110,200]$ \\
\hline \multicolumn{4}{|l|}{ Experimental } \\
\hline EM Scale & 44 & 33 & 25 \\
\hline EM Resolution & 23 & 10 & 4 \\
\hline Recoil Model & 43 & 41 & 35 \\
\hline Efficiencies & 21 & 19 & 19 \\
\hline Backgrounds & 5 & 6 & 9 \\
\hline Experimental Total & 69 & 58 & 48 \\
\hline \multicolumn{4}{|l|}{ W Model } \\
\hline $\mathrm{PDF}$ & 16 & 20 & 24 \\
\hline QED & 7 & 7 & 7 \\
\hline Boson $p_{T}$ & 3 & 1 & 0 \\
\hline$W_{M}$ & 13 & 5 & 2 \\
\hline W model Total & 22 & 22 & 25 \\
\hline Total & 72 & 61 & 54 \\
\hline
\end{tabular}

Table 12.2: Experimental and theoretical systematic uncertainties on the $\mathrm{W}$ boson width measured with $1 \mathrm{fb}^{-1}$ in the $W \rightarrow e \nu$ channel. Fits are performed over three different ranges in the tail of the $M_{\mathrm{T}}$ distribution. 


\section{Chapter 13}

\section{Results}

As discussed in Chapter 3, the measurements of the $W$ boson mass and width were blinded, to prevent bias. A series of cross-checks and comparison plots were produced. Only after we were sufficiently convinced by these checks, did we unblind the results. In this chapter, we present the final, unblinded measurements of the $W$ boson mass and width using the Recoil Library Method, and their respective cross-checks.

\subsection{Final Result for the $W$ Boson Mass}

The final, unblinded mass measurements of the $W$ boson mass, using the Recoil Library Method, are given in Table 13.2. These measurements all give good agreement with the current world average of $80.398 \pm 0.025 \mathrm{GeV}$. They also give good agreement with fits done using our fast MC model with a parameterized recoil model instead of the recoil library method. We present these comparisons as a cross-check in the following section.

\begin{tabular}{|c|c|c|}
\hline Variable & Fitted Mass $[\mathrm{GeV}]$ & Overall Uncertainty $[\mathrm{GeV}]$ \\
\hline$M_{\mathrm{T}}$ & $80.4035 \pm 0.024$ (stat) \pm 0.039 (syst) & 0.046 \\
$p_{\mathrm{T}}(e)$ & $80.4165 \pm 0.027$ (stat) \pm 0.038 (syst) & 0.047 \\
$M E T$ & $80.4025 \pm 0.023$ (stat) \pm 0.043 (syst) & 0.049 \\
\hline
\end{tabular}

Table 13.1: Final result of the data fits for the $\mathrm{W}$ mass, using the recoil library method and the 1 $\mathrm{fb}^{-1}$ of collider data.

\subsection{Cross-Checks and Comparison Plots for $M_{W}$}

Before fitting or even comparing $W \rightarrow e \nu$ observables, it is useful to compare the fast MC model with $Z \rightarrow e e$ data. Figures 13.1, 13.2, and 13.3 shows comparison plots for the invariant 
mass, electron $p_{\mathrm{T}}$ and recoil $p_{\mathrm{T}}$ distributions between $Z \rightarrow e e$ data and fast MC.

Figures 13.4, 13.6, 13.7 show the comparison plots between data and fast MC evaluated at the fit values, for $W \rightarrow e \nu$ observables. The model gives good $\chi^{2}$ comparison with data. Figure 13.5 shows an example of the negative log-likelihood versus $W$ boson mass for the fit to the $M_{\mathrm{T}}$ distribution.

In addition to performing fits on the whole data set, we broke the $W \rightarrow e \nu$ data into subsets with different instantaneous luminosities, run numbers, SET ranges, and $u_{\|}$values, as well as different $\eta_{\text {det }}, U_{\mathrm{T}}$, and $\phi$-mod cuts. For each of these data subsets, it would be proper to first remeasure the energy scale, and generate the fast $\mathrm{MC} W$ templates with the new energy scale. Because this is too time intensive, we proceed as follows. Since the energy scale in the $W$ boson mass measurement at $\mathrm{D} \varnothing$ is set by $Z \rightarrow e e$ data, we are really measuring the ratio of the $W$ boson mass to the already well-known $Z$-boson mass. Even without retuning our $\mathrm{MC}$ for the various data sets, we expect that the $M_{W} / Z_{W}$ should stay relatively constant, since effects like the energy scale will cancel. The relevant observable in our cross-check plots is thus the change in the $M_{W} / Z_{W}$ ratio, shown on the right side of Fig. 13.8-13.12. This is relatively stable and consistent over the data sub-sets with our fit value for the entire data set.

Finally, we compare $M_{W}$ measured using the Recoil Library Method, to the value extracted using the parameterized recoil model. While the recoil model is a small systematic for the $W$ boson mass measurement, we nonetheless lack a good first-principles description of the recoil system. The two methods therefore provide good cross-checks for unaccounted biases that could exist in either model. Table ?? shows the results using the parameterized approach. Both models give good agreement for all three observables. 

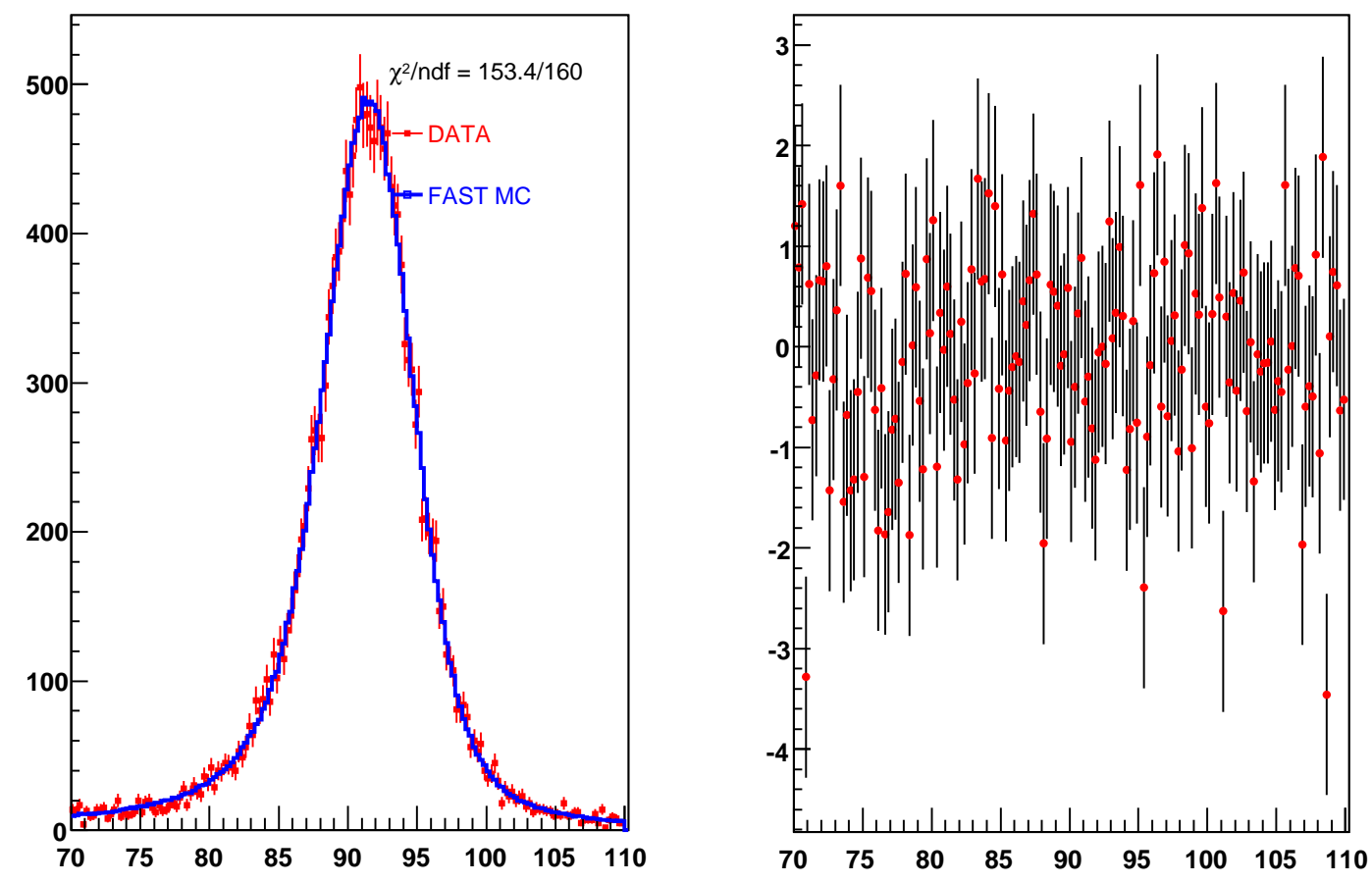

Figure 13.1: Comparison of $Z \rightarrow e e$ invariant mass spectrum between data and fast MC.

\begin{tabular}{|c|c|c|}
\hline Variable & Fitted Mass $[\mathrm{GeV}]$ & Overall Uncertainty $[\mathrm{GeV}]$ \\
\hline$M_{\mathrm{T}}$ & $80.4011 \pm 0.024$ (stat) \pm 0.038 (syst) & 0.043 \\
$p_{\mathrm{T}}(e)$ & $80.4010 \pm 0.027$ (stat) \pm 0.041 (syst) & 0.048 \\
$M E T$ & $80.4012 \pm 0.023$ (stat) \pm 0.044 (syst) & 0.050 \\
\hline
\end{tabular}

Table 13.2: Final result of the data fits for the $\mathrm{W}$ mass, using the parameterized recoil method and the $1 \mathrm{fb}^{-1}$ of collider data. 

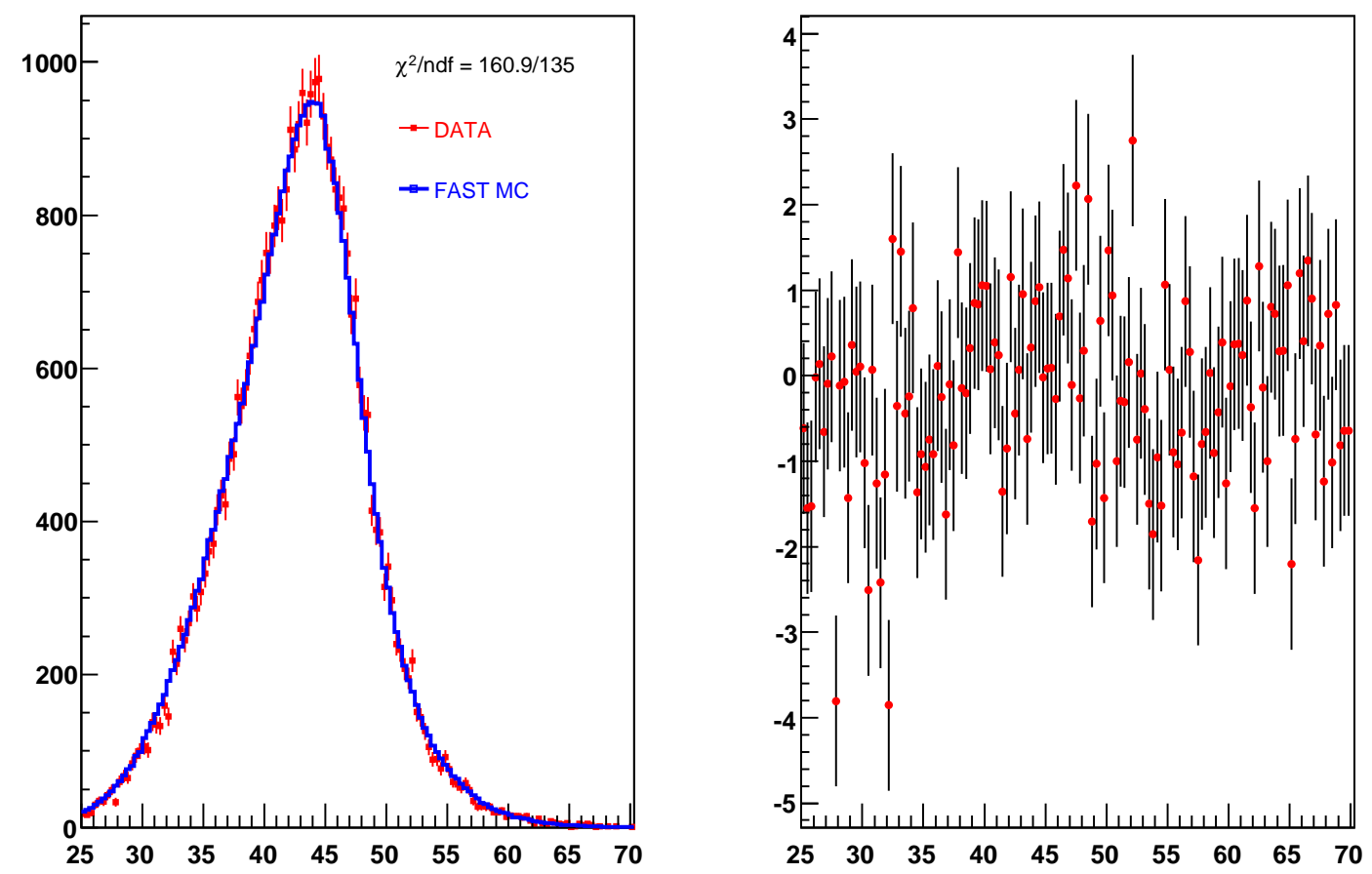

Figure 13.2: Comparison of $Z \rightarrow e e p_{\mathrm{T}}(e)$ spectrum between data and fast MC. 

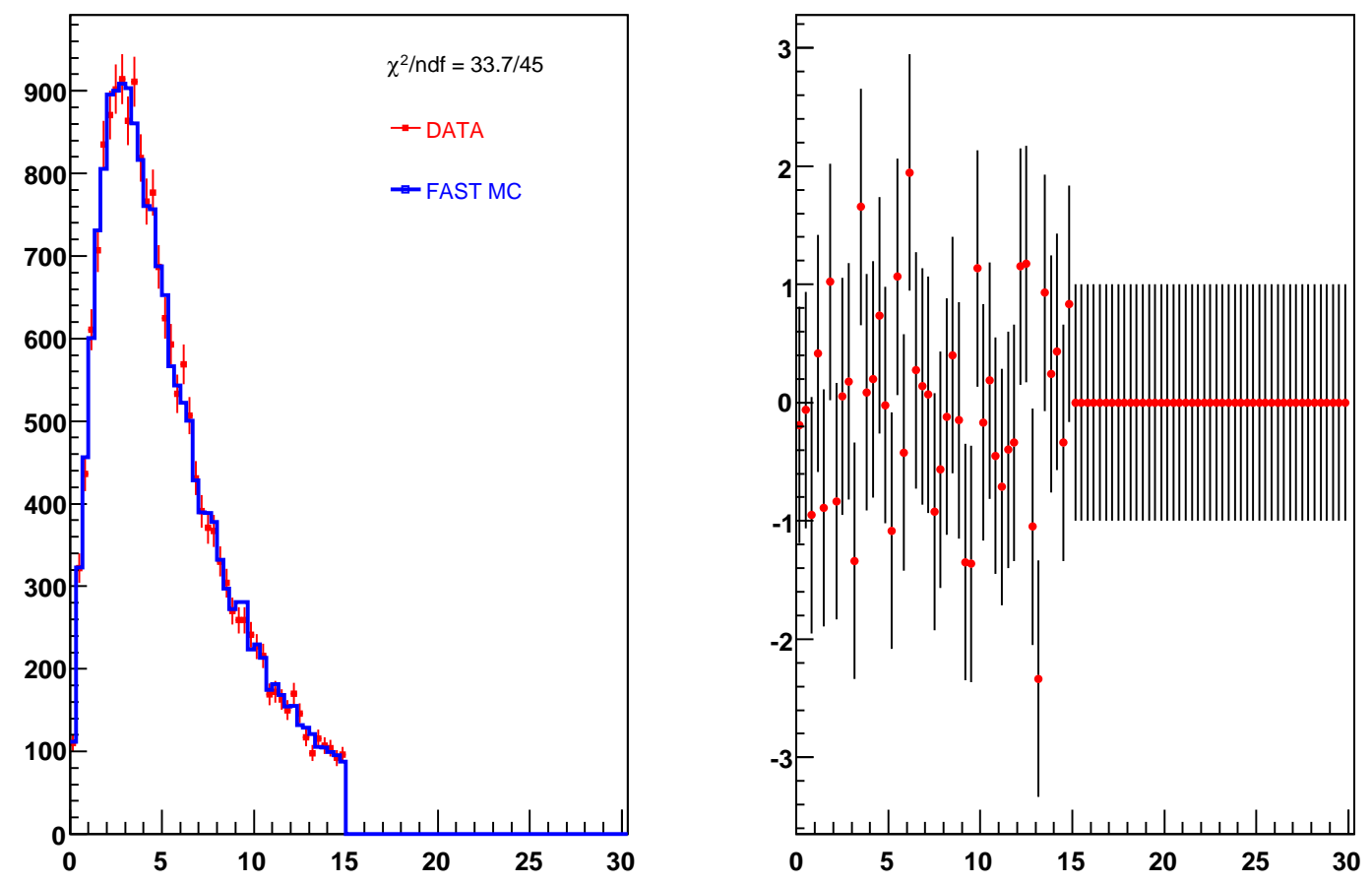

Figure 13.3: Comparison of $Z \rightarrow e e$ recoil $p_{\mathrm{T}}$ spectrum between data and fast MC. 

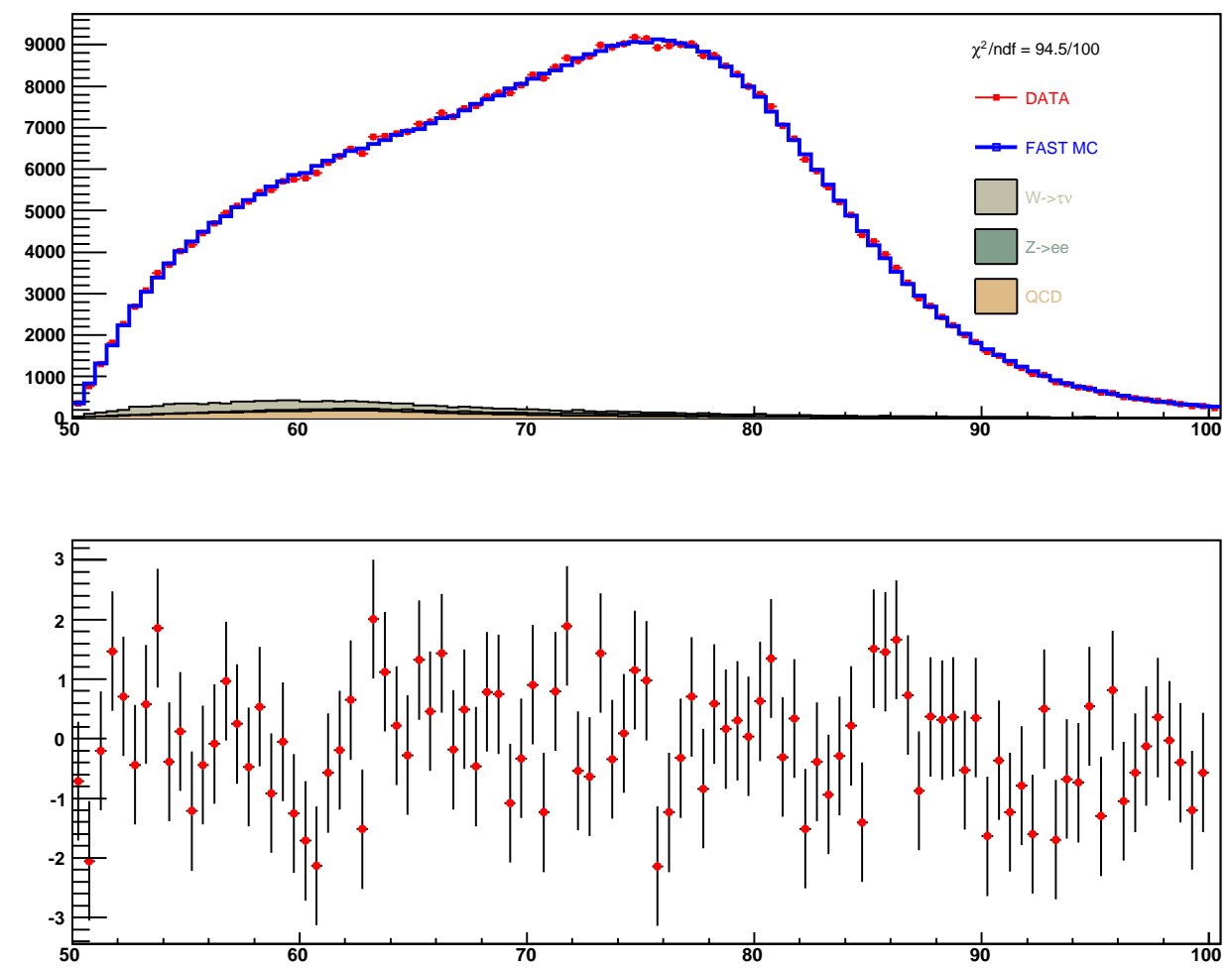

Figure 13.4: Comparison plots between data (points) and fast MC (lines) for the $\mathrm{W} M_{\mathrm{T}}$ distribution. 


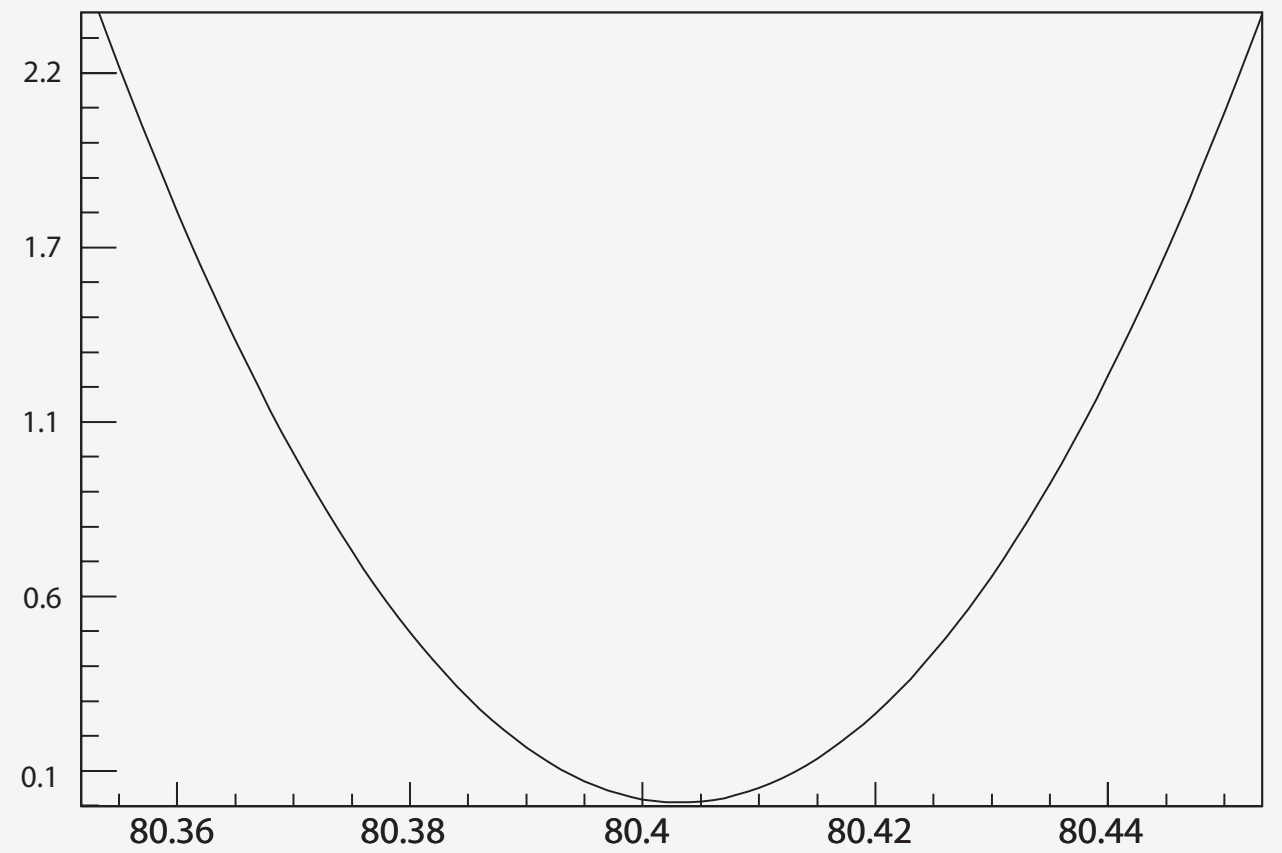

Figure 13.5: Negative log-likelihood plot for the $W$ boson mass fit to the $M_{\mathrm{T}}$ observable. 

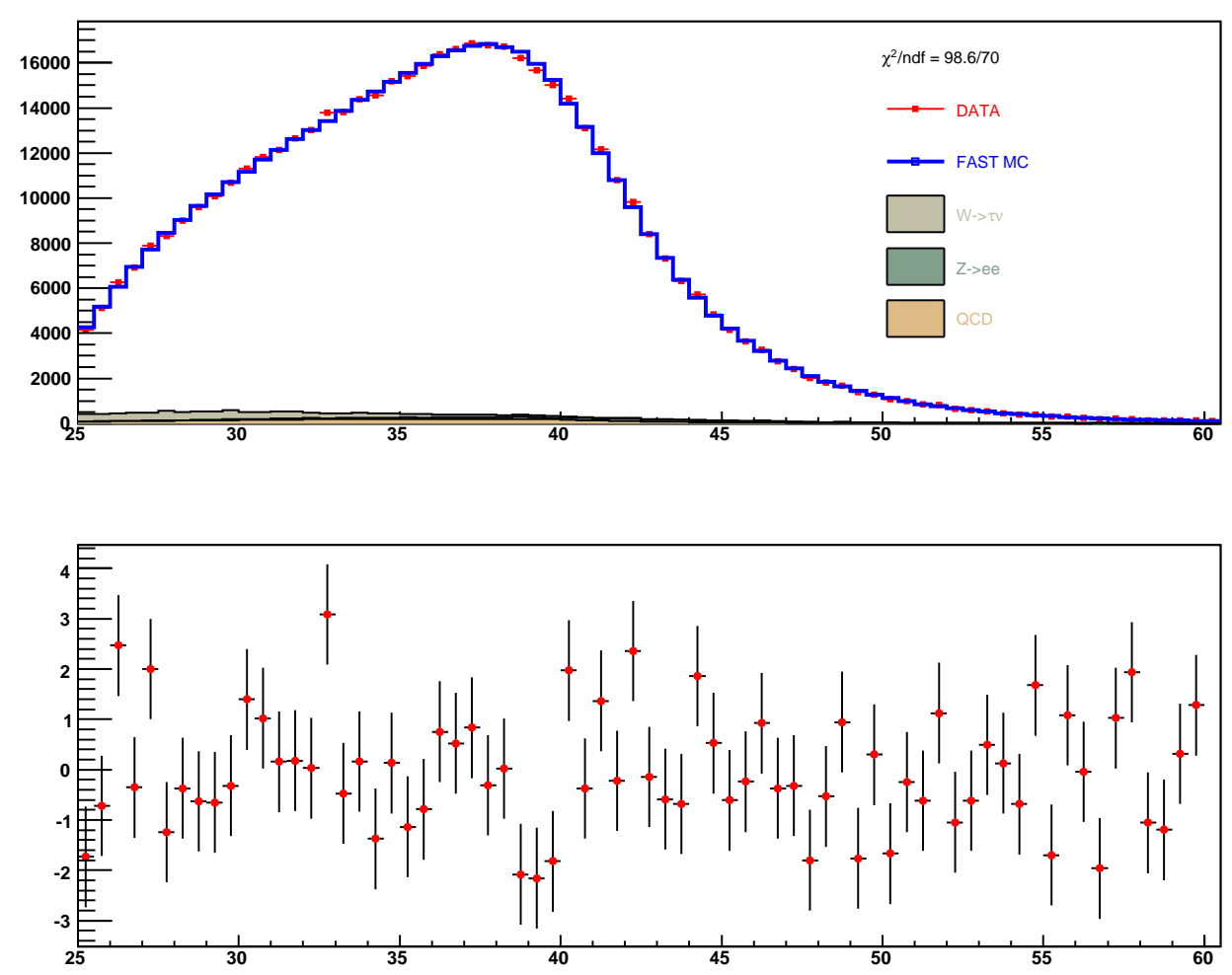

Figure 13.6: Comparison plots between data (points) and fast $\mathrm{MC}$ (lines) for the $p_{\mathrm{T}}(e)$ distribution. 

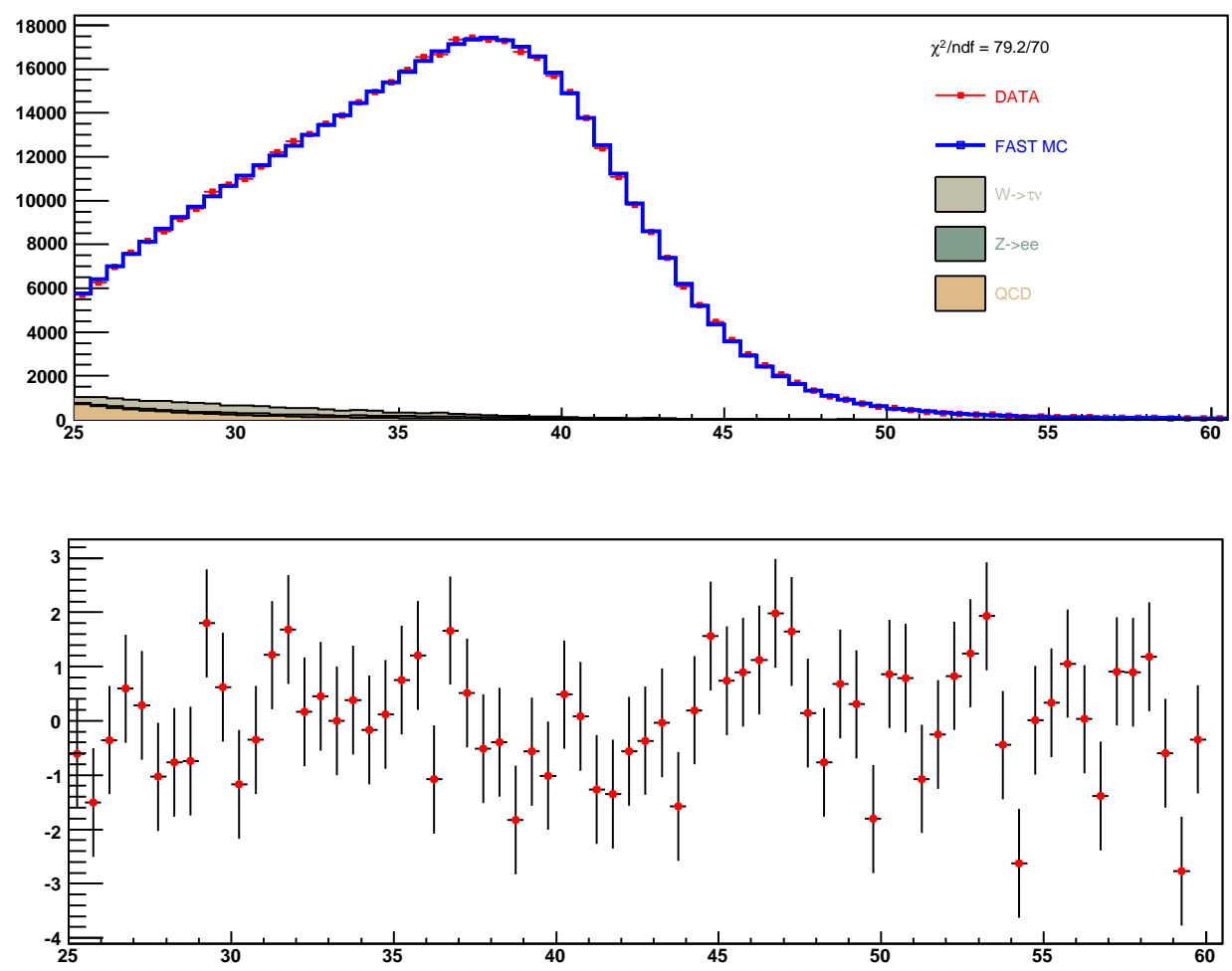

Figure 13.7: Comparison plots between data (points) and fast MC (lines) for the $E_{T}$ distribution.
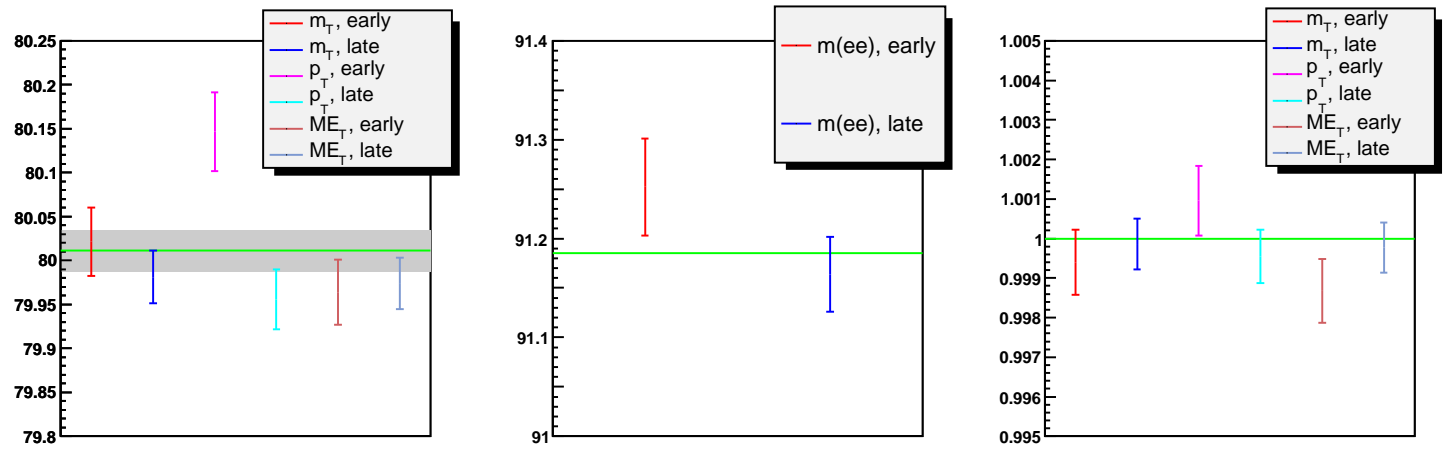

Figure 13.8: Left: $\mathrm{W}$ mass as measured from $M_{\mathrm{T}}, p_{\mathrm{T}}(e)$, and $E_{T}$ observables for two independent run periods. Middle: The equivalent fits for the $\mathrm{Z}$ mass from the di-electron invariant mass spectrum. Right: The fractional change in the $\mathrm{W} / \mathrm{Z}$ mass ratio measured from $M_{\mathrm{T}}, p_{\mathrm{T}}(e)$, and $E_{T}$ observables. The green line represents the nominal value. 

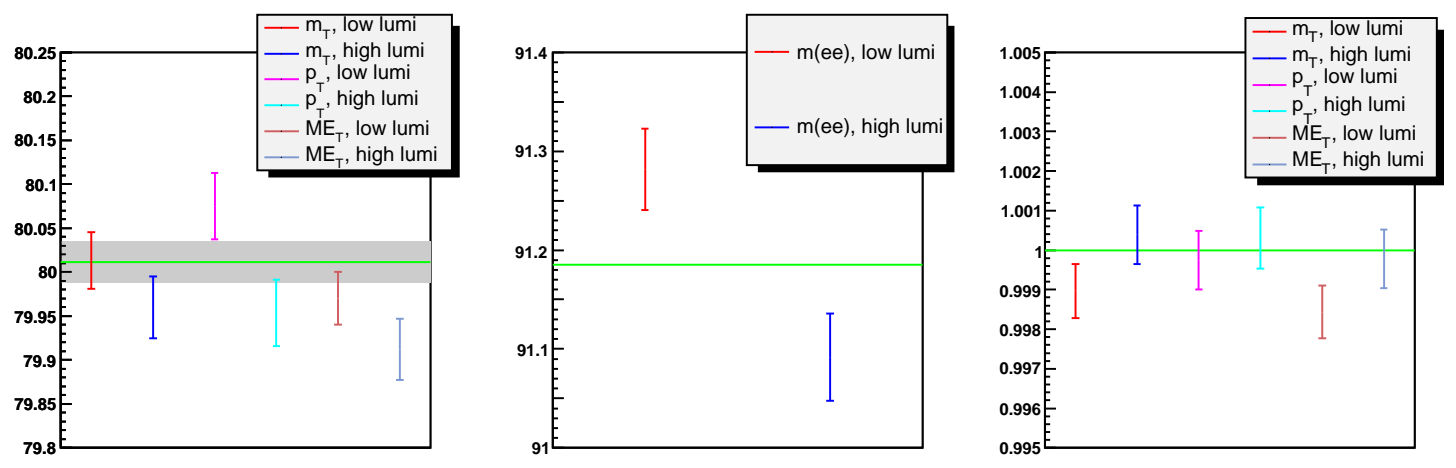

Figure 13.9: Left: $\mathrm{W}$ mass as measured from $M_{\mathrm{T}}, p_{\mathrm{T}}(e)$, and $E_{T}$ observables for two data subsets, corresponding to different luminosities. Middle: The equivalent fits for the $\mathrm{Z}$ mass from the dielectron invariant mass spectrum. Right: The fractional change in the $\mathrm{W} / \mathrm{Z}$ mass ratio measured from $M_{\mathrm{T}}, p_{\mathrm{T}}(e)$, and $E_{T}$ observables. The green line represents the nominal value.
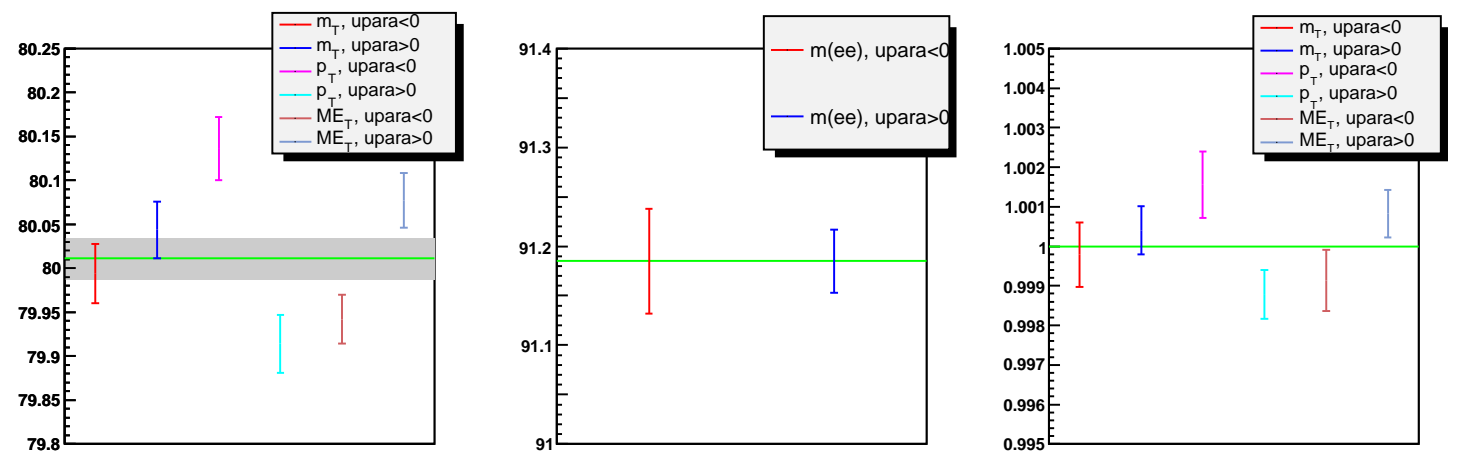

Figure 13.10: Left: $\mathrm{W}$ mass as measured from $M_{\mathrm{T}}, p_{\mathrm{T}}(e)$, and $E_{T}$ observables for two data subsets, corresponding to positive and negative $u_{\|}$. Middle: The equivalent fits for the $\mathrm{Z}$ mass from the dielectron invariant mass spectrum. Right: The fractional change in the $\mathrm{W} / \mathrm{Z}$ mass ratio measured from $M_{\mathrm{T}}, p_{\mathrm{T}}(e)$, and $E_{T}$ observables. The green line represents the nominal value. 

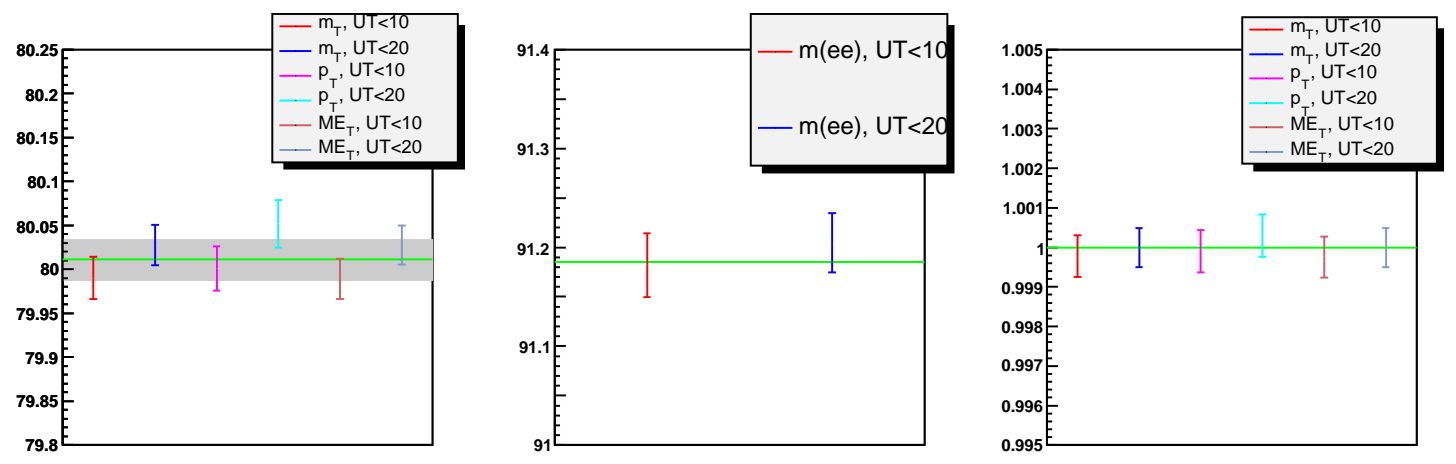

Figure 13.11: Left: $\mathrm{W}$ mass as measured from $M_{\mathrm{T}}, p_{\mathrm{T}}(e)$, and $E_{T}$ observables for two different $U_{T}$ cuts. Middle: The equivalent fits for the $\mathrm{Z}$ mass from the di-electron invariant mass spectrum. Right: The fractional change in the $\mathrm{W} / \mathrm{Z}$ mass ratio measured from $M_{\mathrm{T}}, p_{\mathrm{T}}(e)$, and $E_{T}$ observables. The green line represents the nominal value.
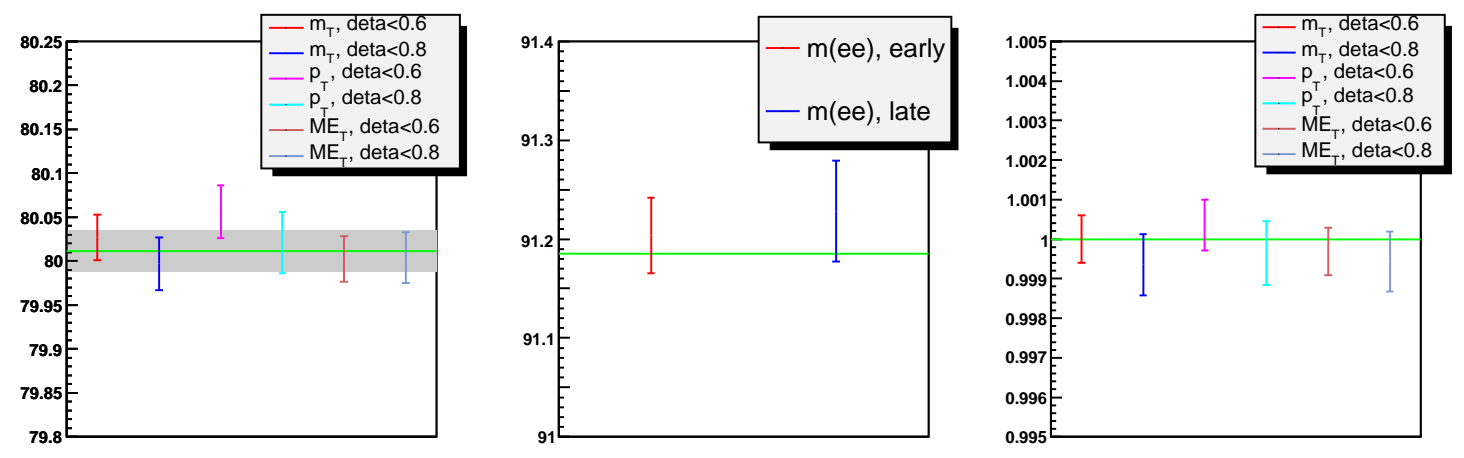

Figure 13.12: Left: $\mathrm{W}$ mass as measured from $M_{\mathrm{T}}, p_{\mathrm{T}}(e)$, and $E_{T}$ observables for two different $\eta_{\text {det }}$ cuts. Middle: The equivalent fits for the $\mathrm{Z}$ mass from the di-electron invariant mass spectrum. Right: The fractional change in the $\mathrm{W} / \mathrm{Z}$ mass ratio measured from $M_{\mathrm{T}}, p_{\mathrm{T}}(e)$, and $E_{T}$ observables. The green line represents the nominal value. 


\subsection{Final Result for the $W$ Boson Decay Width}

The final measurement of the $W$ boson width over the tail of the $M_{\mathrm{T}}$ spectrum, using the Recoil Library Method is $\Gamma_{W}=2.025 \pm 0.039$ (stat) \pm 0.061 (syst). This value is in good agreement with the standard model prediction of $\Gamma_{W}=2.091 \pm 0.002 \mathrm{GeV}$ and the world average of $\Gamma_{W}=2.098 \pm 0.048$

\begin{tabular}{|c|c|c|}
\hline Variable & Fitted Width $[\mathrm{GeV}]$ & Overall Uncertainty $[\mathrm{GeV}]$ \\
\hline$M_{\mathrm{T}}$ & $2.025 \pm 0.039$ (stat) \pm 0.061 (syst) & 0.072 \\
\hline
\end{tabular}

Table 13.3: Final result of data fits for the $\mathrm{W}$ width, using the recoil library method and $1 f b^{-1}$ of collider data.

\subsection{Cross-Checks and Comparison Plots for $\Gamma_{W}$}

Figure 13.13 shows the comparison between the data and fast $\mathrm{MC} M_{\mathrm{T}}$ distribution, evaluated at the fit value for the $W$ boson width. Figure 13.14 shows the same comparison in the tail region used as our fit range $([100,200] \mathrm{GeV})$. Our model generally gives good $\chi^{2}$ agreement with this data observable. Likewise, Fig. 13.15 and 13.16 show comparisons in the tail region of the $p_{\mathrm{T}}(e)$ and $E_{T}$ distributions, respectively. The large $\chi^{2}$ per degree of freedom in the tail of the $E_{T}$ plot is explained by a single bin in the data at $75 \mathrm{GeV}$ that, when removed, brings the $\chi^{2}$ per degree of freedom close to one.

We also fit for $\Gamma_{W}$ in the $p_{\mathrm{T}}(e)$ and $E_{T}$ distributions as a cross-check. Our results are shown in Table 13.4 and agree with the $M_{\mathrm{T}}$ fit to within statistical uncertainties.

The rest of the following consistency checks will be presented in relation to the blinded fit value for the width, which is $2.2736 \mathrm{GeV}$. The blinding offset, now known to be $0.298 \mathrm{GeV}$, is applied to all of the data points in these checks.

As with the $W$ boson mass measurement, we measure the width over various sub-sets of the data. Figure 13.17 show the blinded result of these various measurements drawn with statistical error bars. These measurements over different data sub-sets are consistent with each other and 


\begin{tabular}{|l|l|}
\hline$\Gamma_{W}\left(p_{\mathrm{T}}(e)\right)[\mathrm{GeV}]$ & $\Gamma_{W}\left(E_{T}\right)[\mathrm{GeV}]$ \\
\hline $2.010 \pm 0.046$ (stat) & $2.056 \pm 0.036$ (stat) \\
\hline
\end{tabular}

Table 13.4: Result of fits for $\Gamma_{W}$ to the $p_{\mathrm{T}}(e)$ and $E_{T}$ observables.
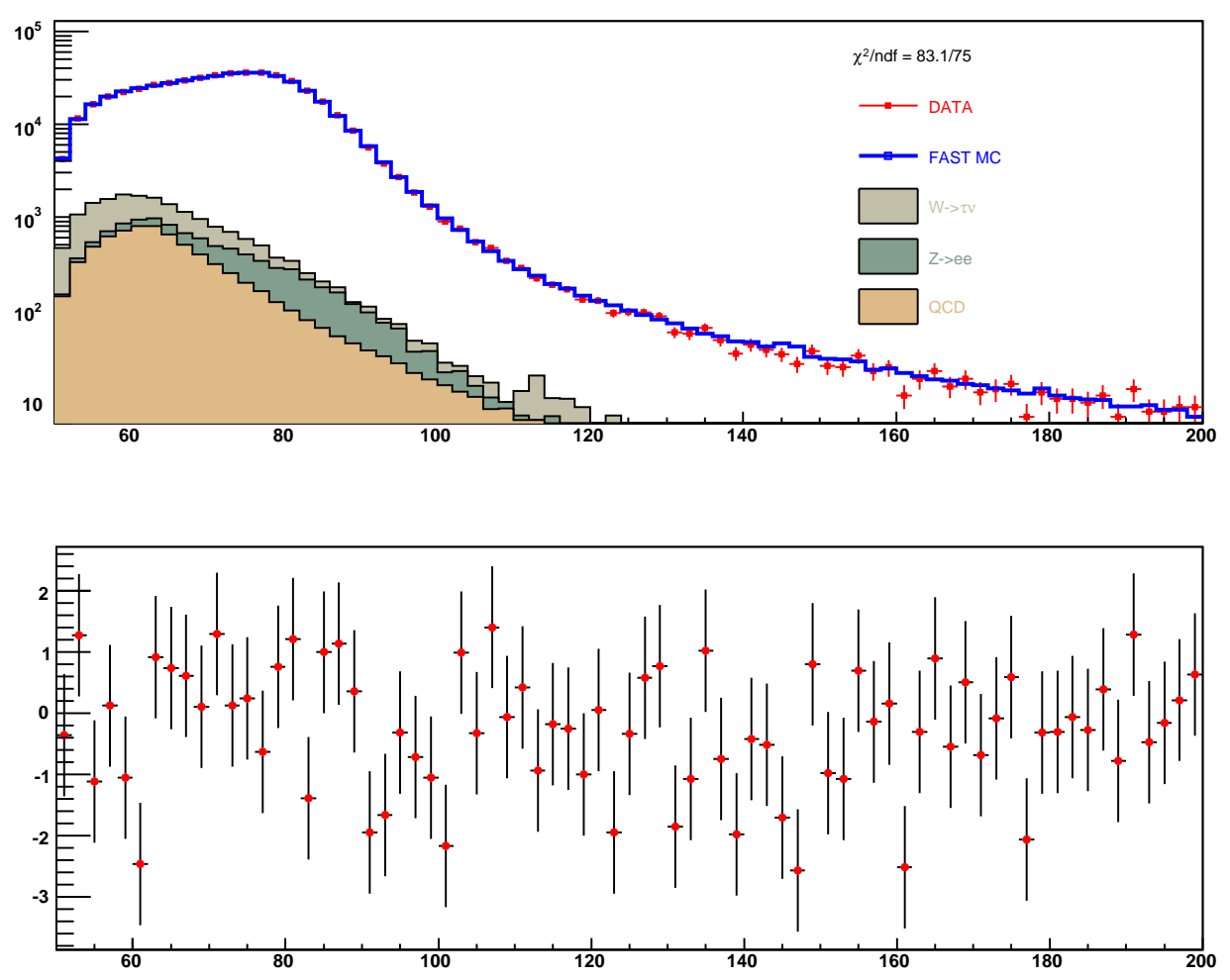

Figure 13.13: Comparison plots between data (points) and fast $\mathrm{MC}$ (lines) for the $\mathrm{W} M_{\mathrm{T}}$ distribution on a $\log$ scale. 

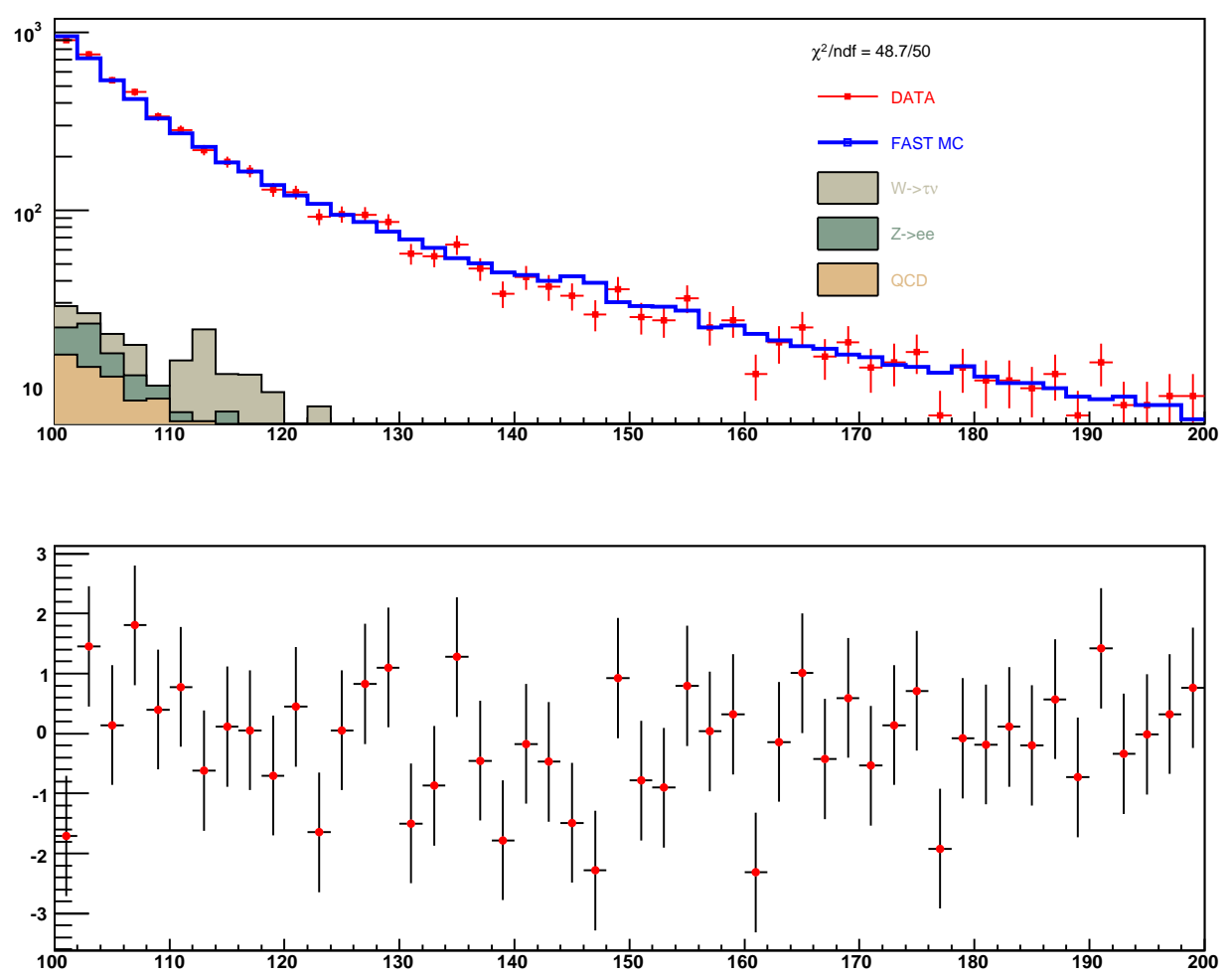

Figure 13.14: Comparison plots between data (points) and fast MC (lines) in the tail of the W $M_{\mathrm{T}}$ distribution on a log scale. 

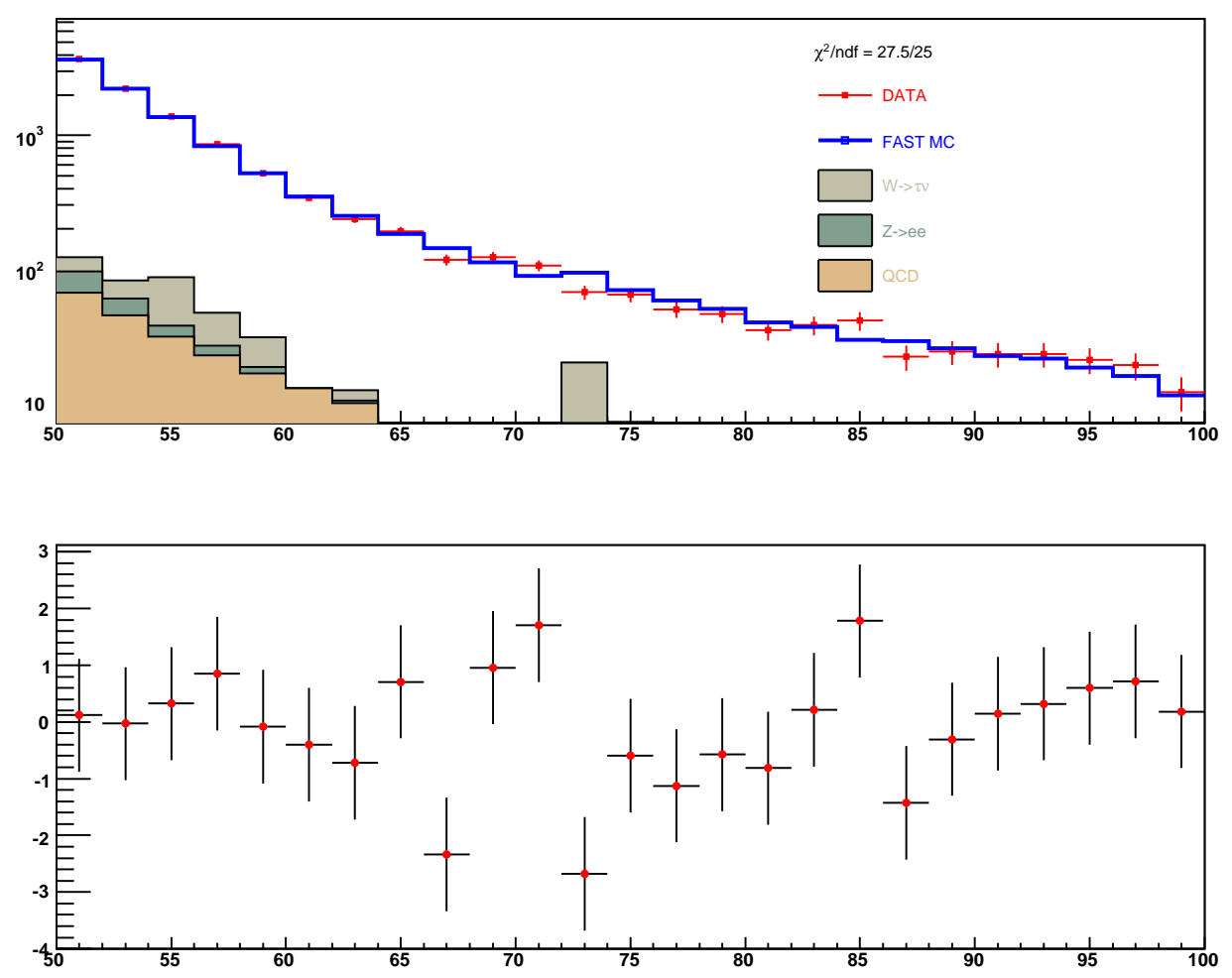

Figure 13.15: Comparison plots between data (points) and fast MC (lines) in the tail of the W $p_{\mathrm{T}}(e)$ distribution on a log scale. 

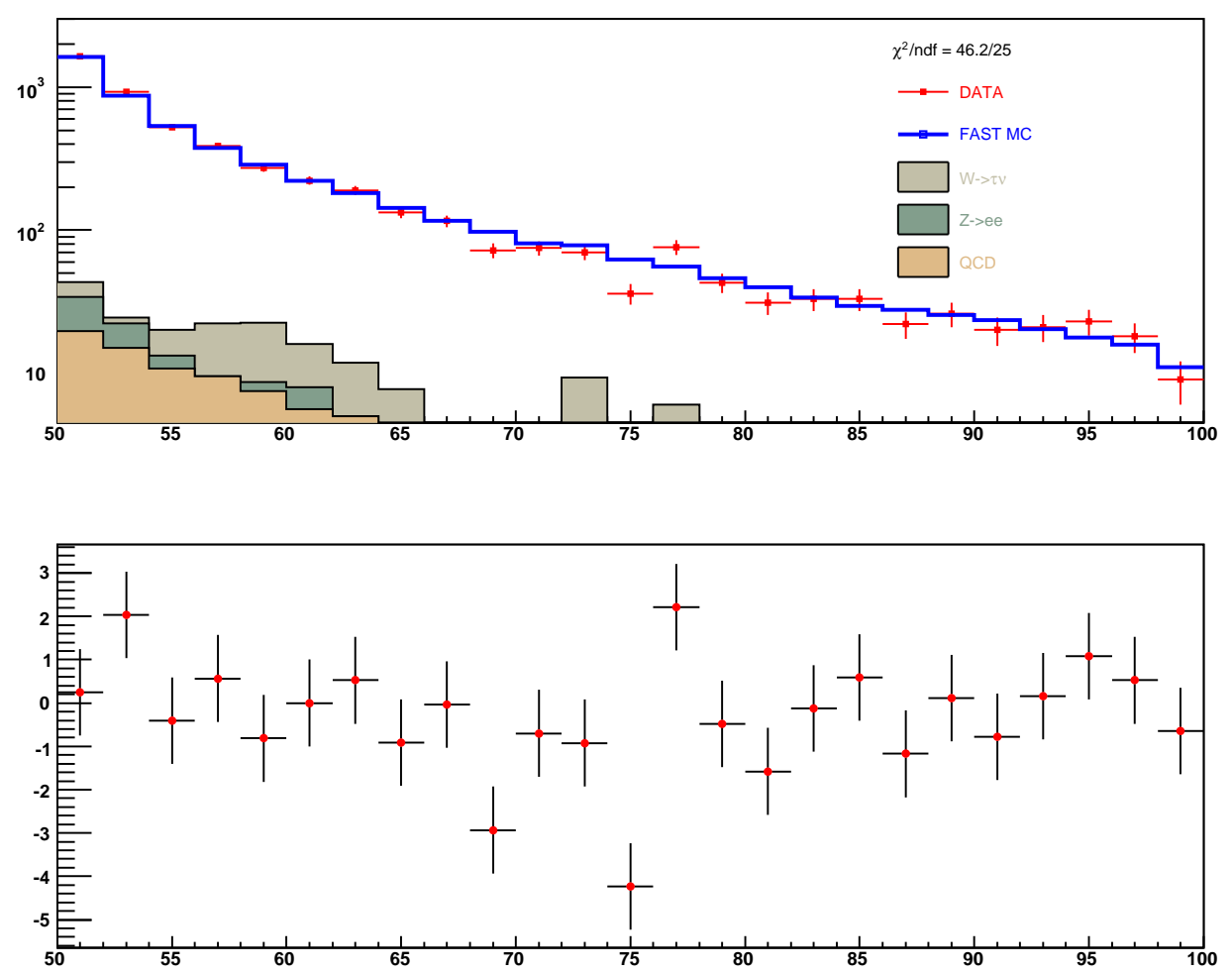

Figure 13.16: Comparison plots between data (points) and fast MC (lines) in the tail of the W $E_{T}$ distribution on a $\log$ scale. 
with the nominal blinded fit value. Since the $W$ boson width is less sensitive to shifts in the $Z$ mass, we do not need to correct for variations on the $\mathrm{Z}$ peak in these cross-check plots.

We also vary the lower and upper limits of the $M_{\mathrm{T}}$ fit. In Fig 13.18 we present the blinded fit results for these variations, with their statistical uncertainties represented by yellow bands. The results are reasonably stable over the different fit ranges.

The tail of the $W M_{\mathrm{T}}$ distribution contains only weak shape information. Consequently, the $W$ boson width can be extracted by comparing the fraction of events in the $M_{\mathrm{T}}$ tail between template distributions and data, with little loss in statistical power. This cross-check gives us a consistent result with that of the negative log-likelihood fit. The blinded results of this simple fit are shown in Fig 13.19

Finally, we compare $\Gamma_{W}$ measured using the Recoil Library Method, to the value extracted using the parameterized recoil model. Since the recoil system contributes the largest systematic uncertainty on the $W$ boson width, this is a very important cross-check. The blinded measurement of $\Gamma_{W}$ extracted from templates made using the parameterized recoil model is $\Gamma_{W}=2.2886 \pm$ 0.038 (stat) \pm 0.062 (syst) $\mathrm{GeV}$, which differs from the Recoil Library Method by only $15 \mathrm{MeV}$. Figure 13.20 shows fit results for different fit ranges for the Recoil Library Method (yellow band), overlaid with the fit results for the parameterized recoil model (red points). The two models give good agreement. 

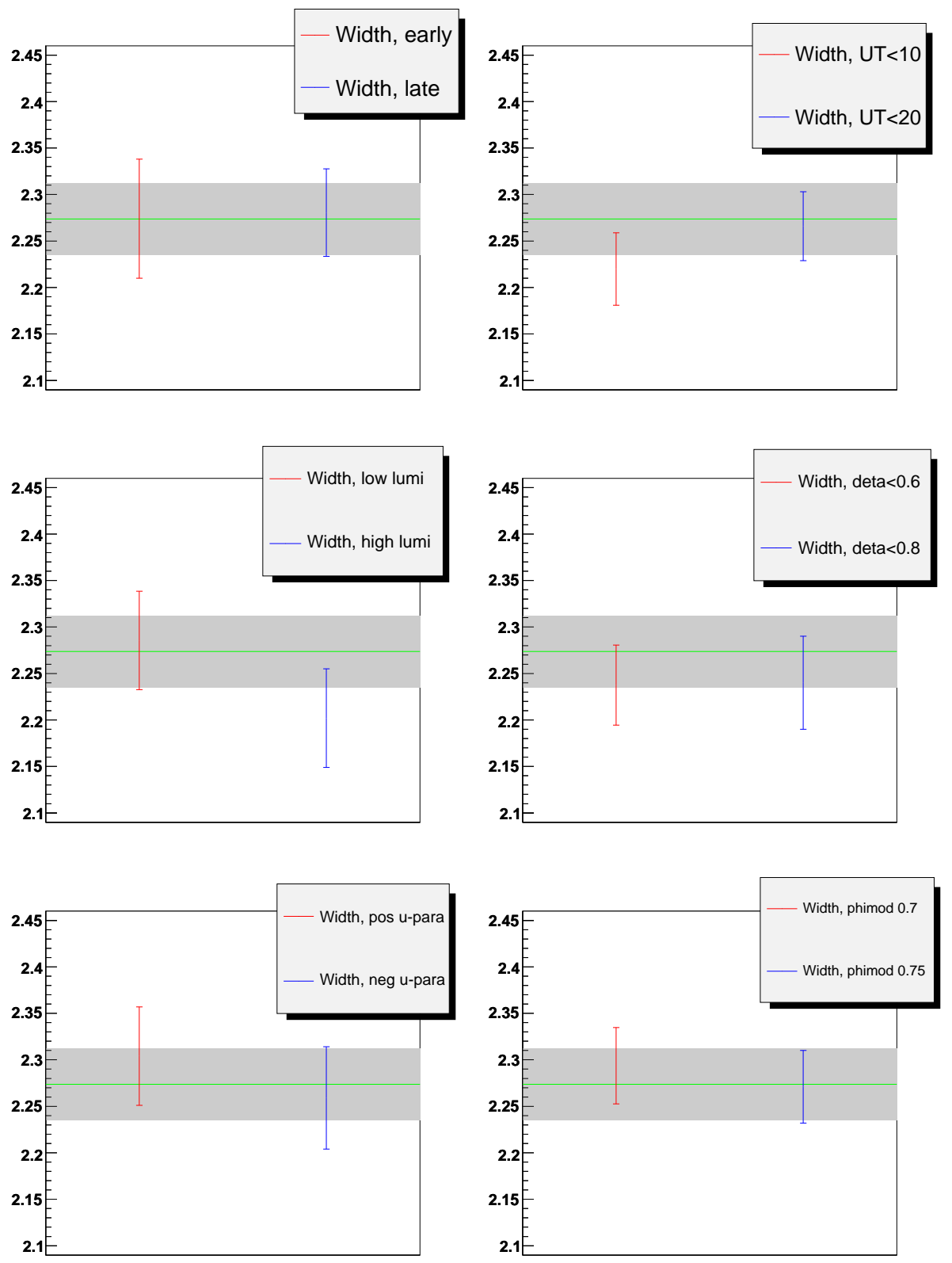

Figure 13.17: Plots showing the blinded $W$ boson width measurements for various sub-sets of the data: separated into independent run-ranges (upper left), for different $U_{T}$ cuts (upper right), separated into independent sets of instantaneous luminosity (middle left), for different $\eta_{\text {det }}$ cuts (middle right), separated into positive and negative $u_{\|}$(lower left), and for different $\phi_{\bmod }$ cuts (lower right). 


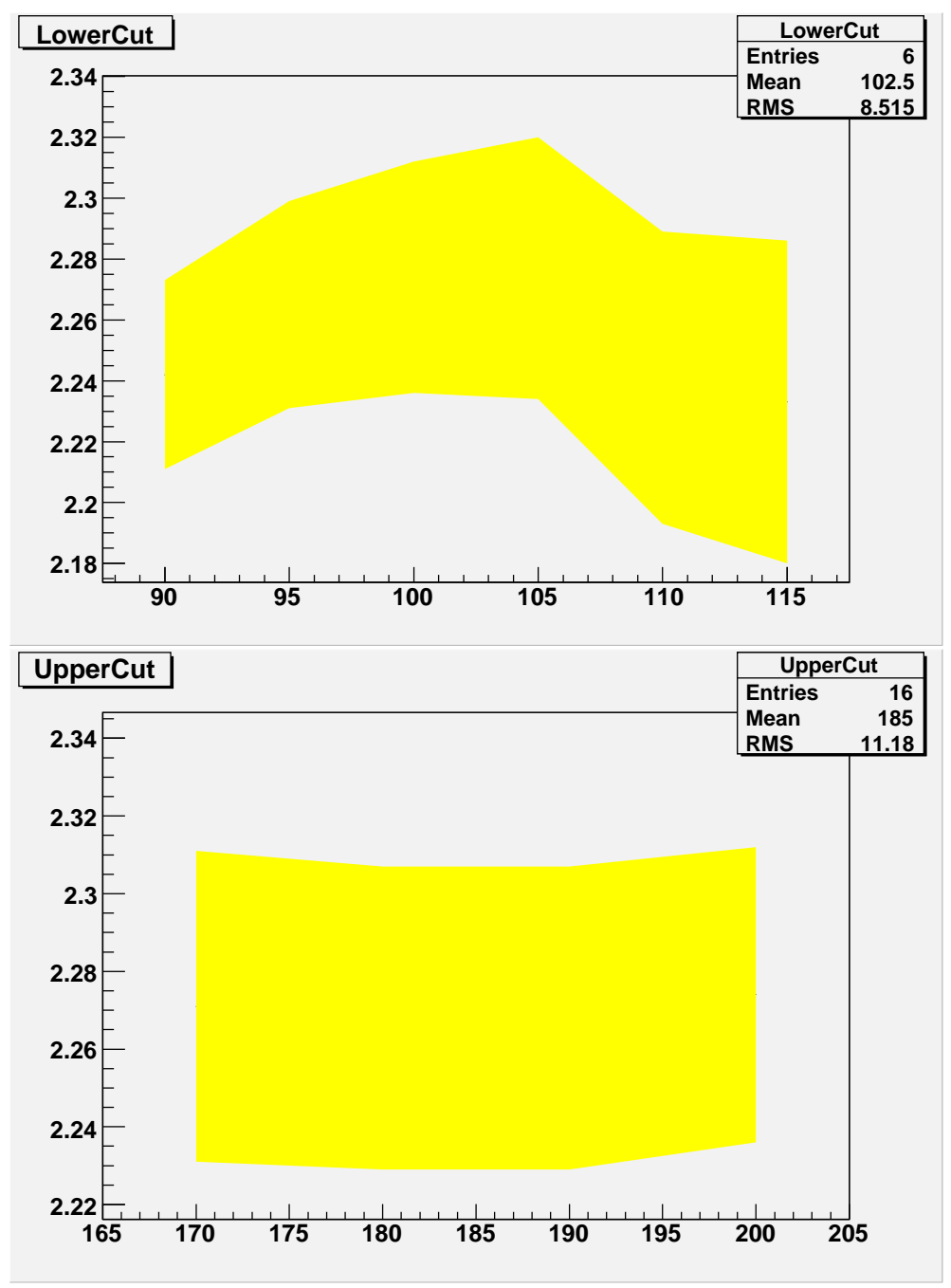

Figure 13.18: Plots showing the variations of the measured $W$ boson width for different values for the lower range of the fit (top) and different values of the upper range (bottom) with statistical error-bars. The yellow bands correspond to the fits using the Recoil Library Method, and the red points correspond to the Parameterized Recoil Model. 


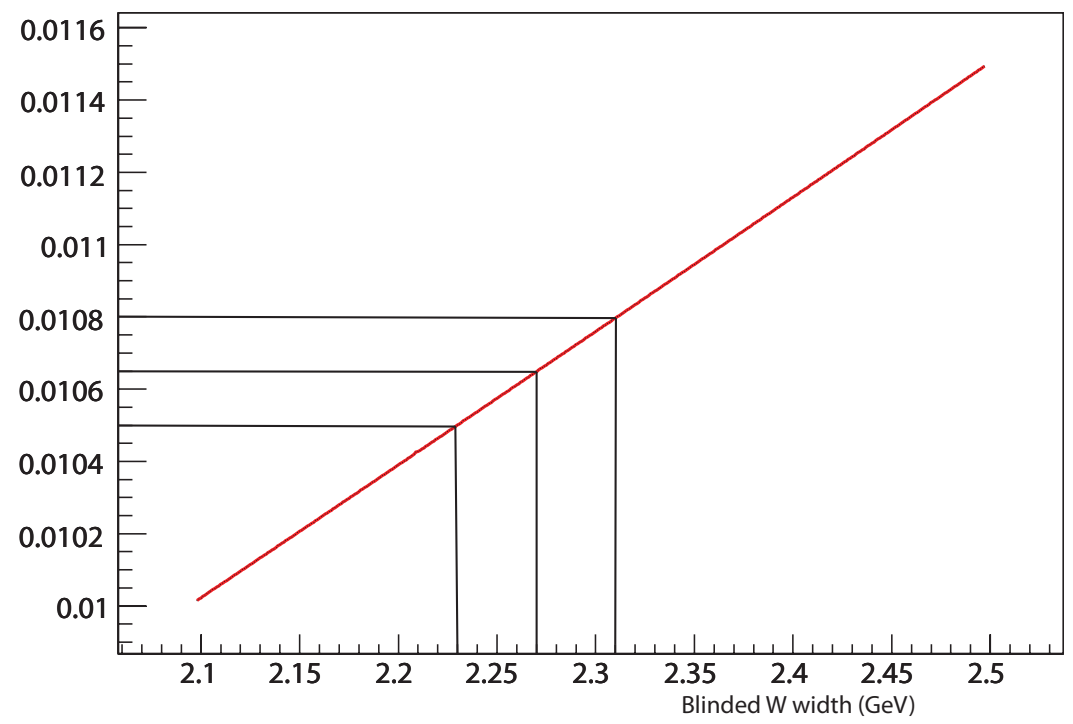

Figure 13.19: Fraction of events in the tail region $[100,200]$ to events in the body $[0,100]$ of the $W$ boson $M_{\mathrm{T}}$ distribution versus blinded $W$ boson width for fast $\mathrm{MC}$ templates. The black lines represent the known tail-to-body ratio for the data, and $\pm 1 \sigma$ of that ratio. The data line intersects with the graph at roughly $2.27 \mathrm{GeV}$, which agrees with the blinded width value obtained through negative log-likelihood fits. 


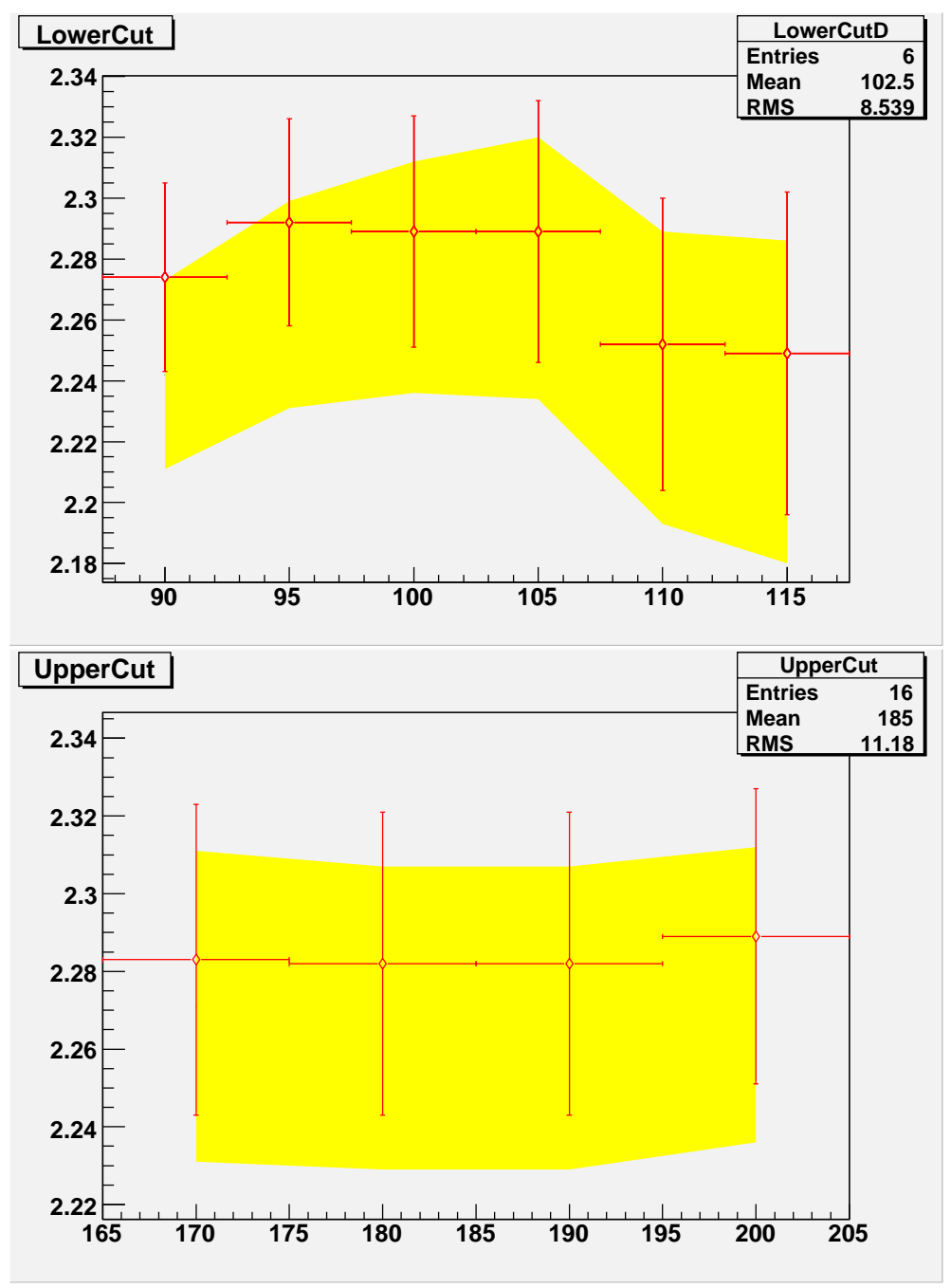

Figure 13.20: Plots showing the variations of the measured $W$ boson width for different values for the lower range of the fit (top) and different values of the upper range (bottom) with statistical error-bars. The yellow bands correspond to the fits using the Recoil Library Method, and the red points correspond to the Parameterized Recoil Model. 


\section{Chapter 14}

\section{Conclusions and Future Prospects}

In this dissertation, we presented measurements of the $W$ boson mass and decay width in 1 $\mathrm{fb}^{-1}$ of data, using a novel method to model the hadronic recoil system in $W$ events using a $Z \rightarrow e e$ data recoil library. The analysis with this Recoil Library Method gave comparable systematic and statistical uncertainties to the parameterized approach for both $M_{W}$ and $\Gamma_{W}$. Our $W$ boson mass and width fits to full MC $W \rightarrow e \nu$ samples gave excellent agreement with the input parameters, to within statistical errors, and provided confidence in the methodology. Our data measurements of both parameters were consistent over various run periods, luminosities, kinematic cuts, and other observables, as well as for different fit ranges. Results for both the $W$ boson mass and width using the parameterized and recoil library approaches were within $15 \mathrm{MeV}$ of each other and smaller than the statistical uncertainties.

These measurements using either recoil method represent the most precise single measurements of $M_{W}$ and $\Gamma_{W}$ to date. They will most certainly reduce the uncertainty on the world averages, which remain stable after including these measurements. These results promise to further restrict the expected mass range of the Standard Model Higgs boson. Figures 14.1 and 14.2 show the measurements made in this thesis compared with the world average and other experimental results for the $W$ boson mass and width, respectively. Figure 14.3 shows the ever more restricted region where the mass of the Higgs boson is allowed by Standard Model calculations.

The method presented in this paper has many advantages. It accurately describes the highly complicated hadronic response and resolution for $W$ recoils in a given calorimeter. It includes complex correlations between the "hard" and "soft" components of the recoil, and scales the recoil appropriately with luminosity. It requires few assumptions, no first-principles description of the recoil system, and no adjustable parameters. As hadron collider experiments such as the Run II Tevatron and the LHC operate at ever higher luminosities, this type of heuristic approach to 


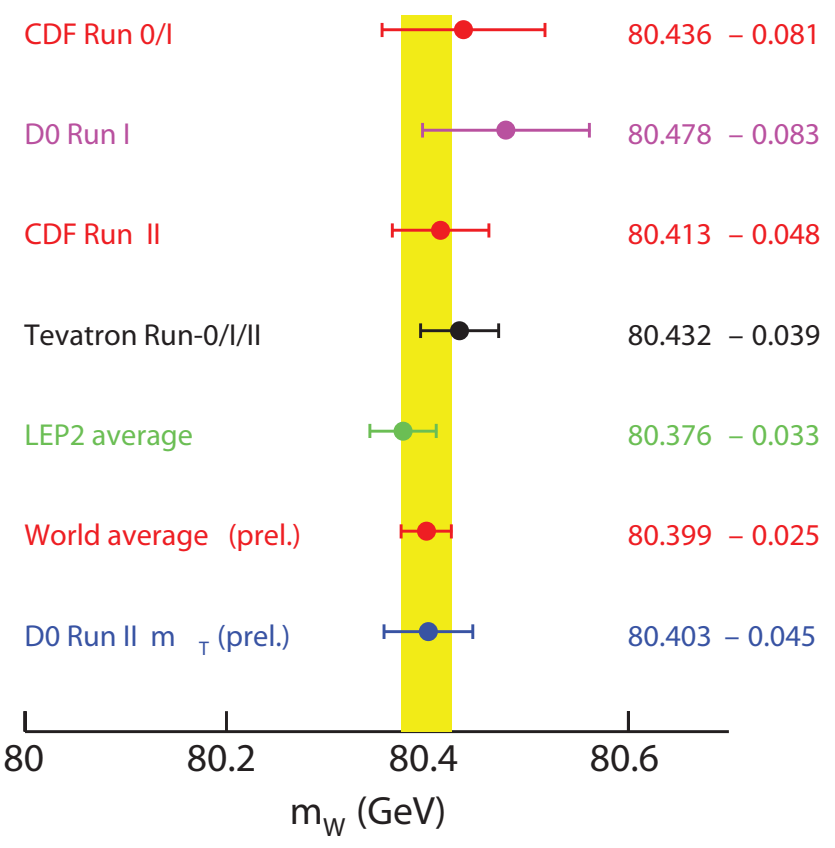

Figure 14.1: The new D0 $W$ boson mass measurement compared with various other measurements and the updated world average (yellow band). 


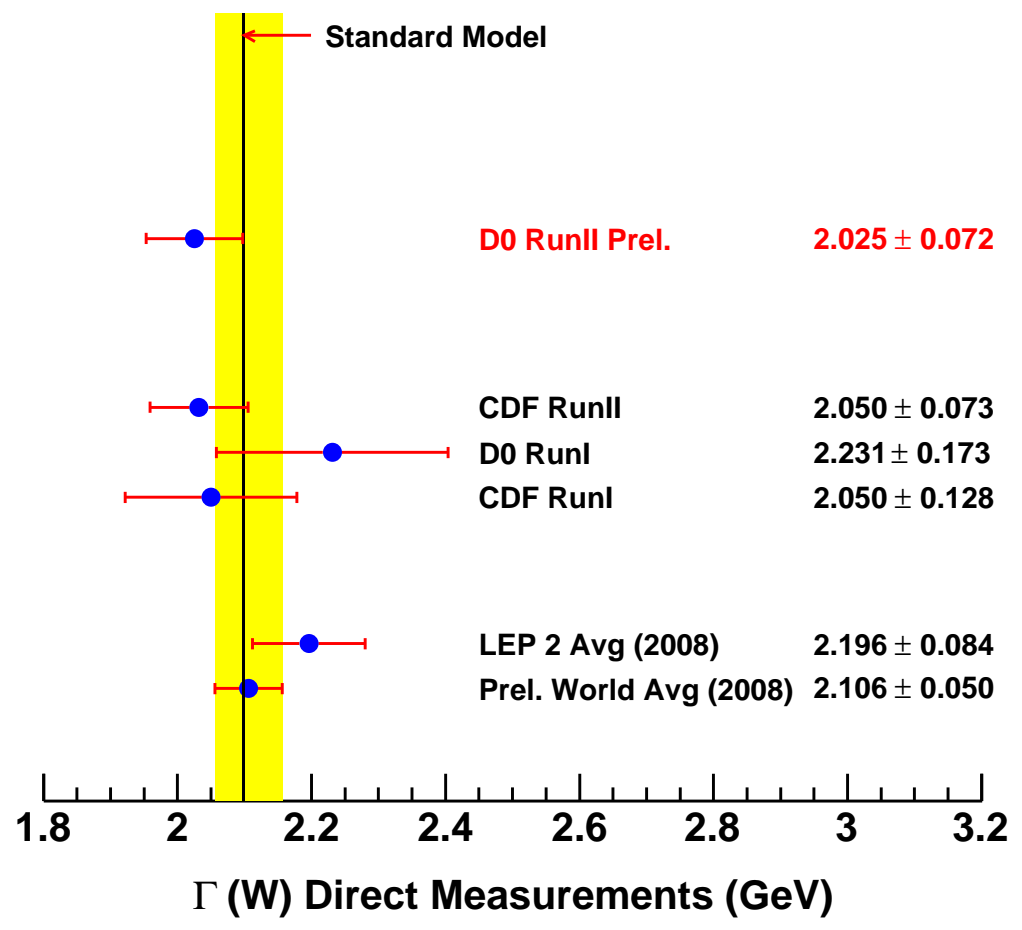

Figure 14.2: The new D0 $W$ boson width measurement compared with various other measurements and the current world average (yellow band). 


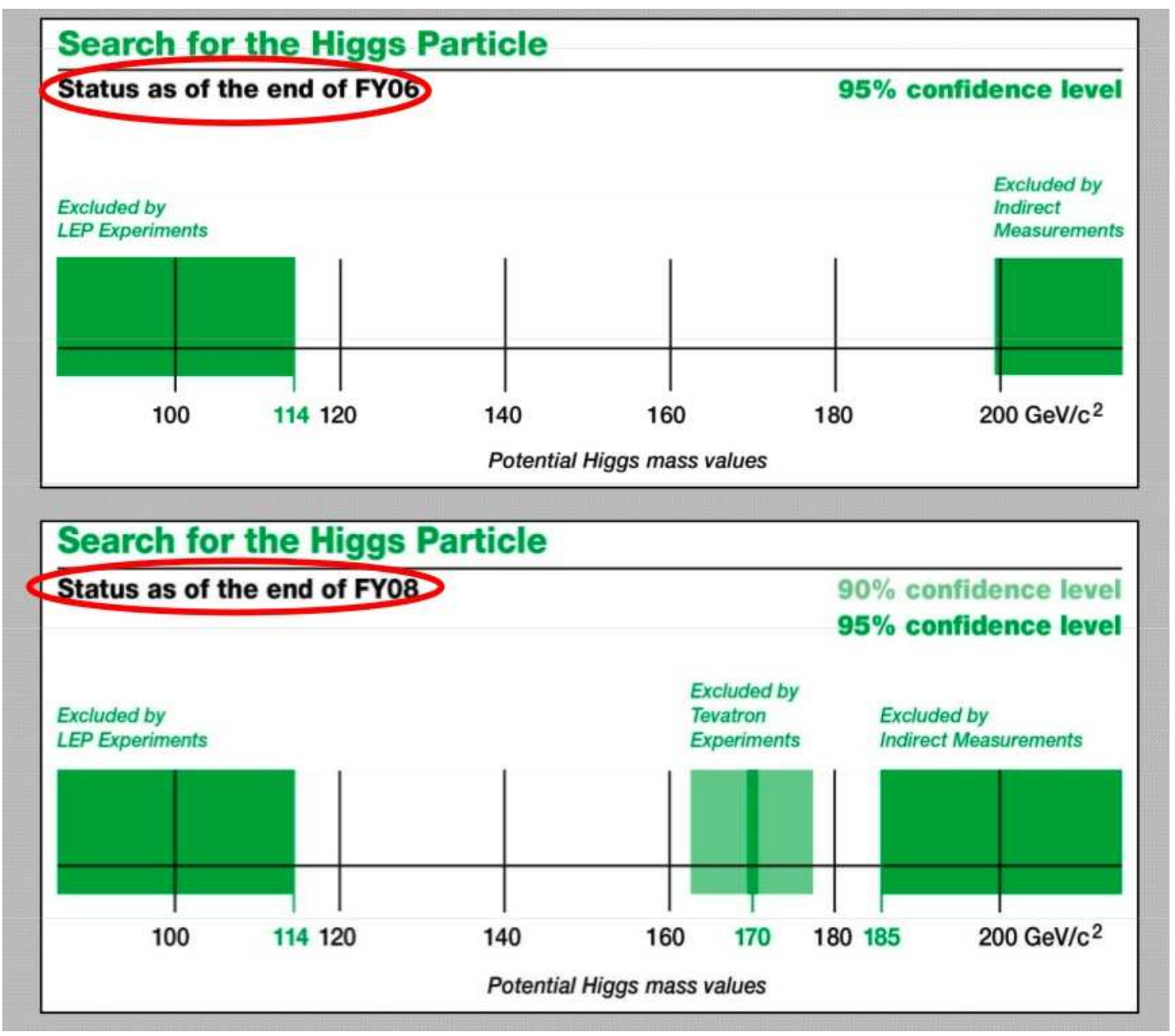

Figure 14.3: Plots showing the restricted regions where the Higgs mass is expected. 
modeling the recoil system will serve as a useful cross-check and alternative to more traditional parametric approaches.

Perhaps most exciting about this analysis are the future prospects. The dominant systematic uncertainties on the $W$ boson mass and width -the electron energy scale and recoil model- are mainly limited by the statistics of the $Z \rightarrow e e$ sample. In the combined $5 \mathrm{fb}^{-1}$ of Run IIb data, these errors will likely shrink by a factor of $\sqrt{5}$, putting the prospect of a $W$ boson mass measurement with $25 \mathrm{MeV}$ total uncertainty well within sight. Comparable results from CDF could, when combined with DØ bring the uncertainty of the Tevatron measurement down to 15 $\mathrm{MeV}$ on the $W$ boson mass, where theoretical uncertainties begin to dominate and constraints on both the Higgs boson and on physics beyond the Standard Model become very interesting. 


\section{Appendix A}

\section{Propagating the Effects of FSR in the Recoil File to $W \rightarrow e \nu$ Templates}

As we have described in Chapter 9, roughly $6 \%$ of the events in the data recoil file contain FSR photons that have not been merged with the corresponding electron and are falsely attributed to the recoil energy. We address this by tightening the lower cut on the $Z$ boson masses for the recoil file construction to $85 \mathrm{GeV}$, thereby removing some of the events with the most energetic photons and reducing the total number of unmerged FSR events by more than $25 \%$. Our final bias due to FSR is measured to be small $(<10 \mathrm{MeV})$. Nonetheless, the remaining events in the recoil file contain some events with unmerged final-state photons at high transverse momenta $(\mathcal{O}(10)$ $\mathrm{GeV})$. It is not intuitive that the final FSR bias on our method should be small. In this appendix we explore why the FSR bias is so low.

To first order, we can approximate the bias on the $W$ boson mass due to FSR in terms of a shift in average $u_{\|}$due to the unmerged photons. However, we cannot attribute this bias to the shift in the $u_{\|}$distribution of the $Z$ recoil file itself, since we care about the effects on the $u_{\|}$distribution of the modeled $W \rightarrow e \nu$ events. These generated $W$ events decay independently

of the $Z$ events from which the recoils are extracted. Instead, we generate $W u_{\|}$distributions, using recoil files with and without FSR photons. We produce these toy distributions by taking a sample of generator-level $W$ events, choosing a random recoil from the recoil file that matches the $W p_{\mathrm{T}}$, and projecting that recoil on to the direction of the generator level electron. The observed shift in the mean values of the $u_{\|}$distributions made from a recoil library with and without FSR photons is consistent with zero, to within the $1.8 \mathrm{MeV}$ error on the means of the distributions. As large as the $p_{T}$ of the unmerged photons can be, the direction of these photons relative to the independently generated $W$ electron is relatively uniform, so the effect cancels on average.

We can also study the observed impact of the unmerged photons on the hadronic energy scale of the recoil file. For low $Z p_{\mathrm{T}}$ we expect little correlation between the direction of the boson 
and the decay electrons. Likewise, we expect little correlation between the radiated photons and $Z$ direction. Thus, at low transverse momenta, the relative directions of FSR photons should cancel and have no net effect on the recoil $p_{\mathrm{T}}$. As $Z p_{\mathrm{T}}$ increases, the electrons and final state photons will be closer to the $Z$ direction. We can plot the projection of the final-state photon $p_{\mathrm{T}}$ along the $Z$ boson direction as a function of $Z p_{\mathrm{T}}$, as shown in Fig A.1. We see that the fitted slope of the graph is $1.2 \%$, reducing the recoil response for those events with unmerged final-state photons. Since those events, after the $85 \mathrm{GeV} Z$ boson mass cut, comprise only $4 \%$ of the total events in the recoil file, we expect the bias on the overall recoil response to be approximately $1.2 \% \times 4 \%=0.05 \%$. For the parameterized recoil model, the derivative of the $M_{\mathrm{T}} W$ boson mass fit with respect to the hadronic energy scale has a slope of $-482 \mathrm{MeV}$. This would give a bias of less than one MeV for a shift in the energy scale of 0.0005 .

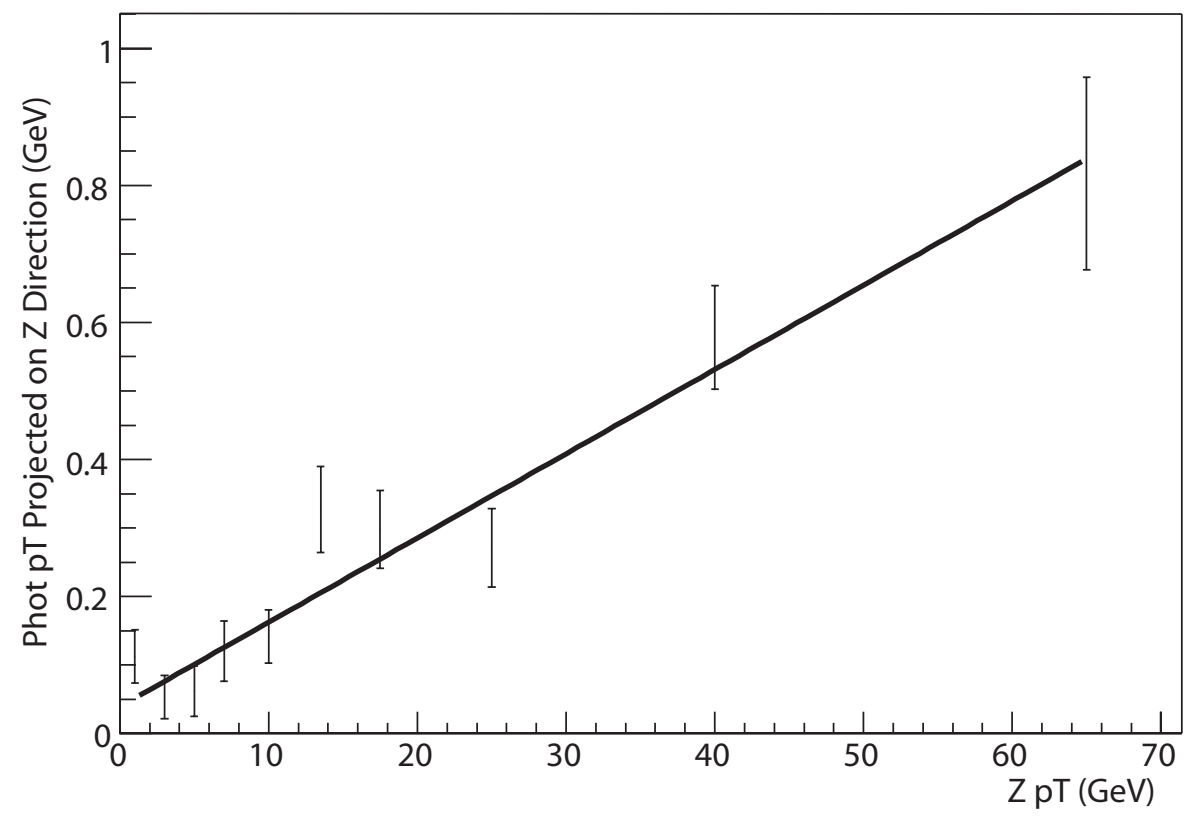

Figure A.1: The projection of unmerged photon $p_{T}$ along the direction of the $Z$ boson, versus the $p_{T}$ of the $Z$. 


\section{A.1 Statistical Power and the Continuity Assumption}

The recoil library method described in this paper assigns each generated $W$ candidate a recoil chosen from a discrete bin in boson $p_{T}$. One would expect the effective recoil response of each bin to fluctuate randomly due to the finite statistics particular to that bin. In contrast, the parameterized recoil model fits all of the data with a single, continuous function. The uncertainty at each point of this smooth function is determined by the uncertainties on the fit parameters which are constrained by the statistics of the whole data set, not just the statistics at that particular point. One might expect that by forgoting this continuity assumption, the recoil library method sacrifices a significant measure of statistical power due to bin-by-bin fluctuations. However, our uncertainty analysis described in Section 9.5.1 does not find this to be the case. To within the statistical precision of our study, the uncertainties are indistinguishable from those of the parameterized model. In this appendix we construct a simple toy study and find that the loss of statistical power from dropping the continuity assumption is small in comparison with the finite hadronic resolution of the D0 detector.

For this study we generate sets of $18 \mathrm{k} Z \rightarrow$ ee recoils according to $1 \mathrm{fb}^{-1}$ of data using a simple toy model. The $p_{T}^{Z}$ spectrum is generated according to a Landau distribution with a mean of $3 \mathrm{GeV}$ and an RMS of $1.5 \mathrm{GeV}$. The maximum boson $p_{T}$ is restricted to $20 \mathrm{GeV}$. The magnitude of the recoil system is smeared according to a Gaussian with a sampling resolution of $0.8 \sqrt{\mathrm{GeV}}$, which roughly agrees with the hadronic resolution of the D0 detector for low $p_{T}$ jets. The hadronic response is assumed to be 1 . The $Z$ recoil information is stored in a histogram with the true $p_{T}^{Z}$ on the $x$-axis and the average measured $u_{T}$ on the $y$-axis. We either use this histogram as a discrete look-up table to simulate the hadronic response (binned approach) or we fit the histogram with a linear function and use the fitted function to simulate the hadronic response (parameterized approach). The two different approaches are similar to the recoil library method and the parameterized recoil method respectively.

We produce 1000 toy recoil histograms using $18 \mathrm{M} Z$ events with $18 \mathrm{k}$ events per toy. For each of the 1000 recoil histograms, we generate $4 \mathrm{M} W$ events with the same $p_{T}$ spectrum as the 
toy recoils described above. Each $W$ boson is assumed to have a mass of $80 \mathrm{GeV}$ and rapidity less than 1.1. We decay the boson into two massless leptons, with a uniform $\phi$ distribution in the rest frame. The two leptons are then boost by the boson $p_{T}$ to the lab frame.

The recoil response for each of the 1000 independent $W$ samples is modeled using either the binned approach or the parameterized approach. We repeat this entire study twice, once without applying a hadronic resolution, and once with an $0.8 \sqrt{\mathrm{GeV}}$ hadronic sampling resolution.

For each toy $W$ sample we construct an " $\eta$-imbalance" distribution, defined as the difference between magnitudes of the boson $p_{T}$ and the projection of the recoil $u_{T}$ along the boson direction. The mean and RMS of the $\eta$-imbalance distribution represents the hadronic response and resolution respectively. When the parameterized approach is used, the $\eta$-imbalance distribution from one $W$ toy is essentially a $\delta$ function with negligible width. If we use the binned approach, the RMS of the $\eta$-imbalance distribution is significantly higher due to both the fluctuation of the mean recoil response averaged over all bins and from the fluctuations of each individual bin around that average response. The effect of these bin-by-bin fluctuations is an over-smearing of the recoil, degrading the statistical power of our model.

The mean value of the $\eta$-imbalance distribution for one toy can fluctuate from zero due to finite $Z$ statistics, over many such toys these fluctuations should cancel. Indeed, in Fig A.2 we see that the average mean of the mean $\eta$-imbalances is consistent with zero for both the parameterized and binned approaches. The RMS of the mean $\eta$-imbalances represents the uncertainty on the average recoil response due to finite $Z$ statistics. We see that the RMS of the mean $\eta$-imbalances over 1000 toys is the same for both approaches. This indicates that both approaches describe the average recoil response function with equivalent statistical power.

It is not obvious that the uncertainty on the mean response should be the same for both approaches. Indeed, the $Z$ recoil library is a special case. The uncertainty on the mean response in the recoil model is equivalent to the average uncertainty on each bin, weighted by the frequency with which recoils are taken from that $p_{T}$ bin. Because the $Z$ and $W$ bosons have very similar $p_{T}$ spectra, the most frequently chosen $W$ recoils correspond to the bins with the most $Z$ recoil 

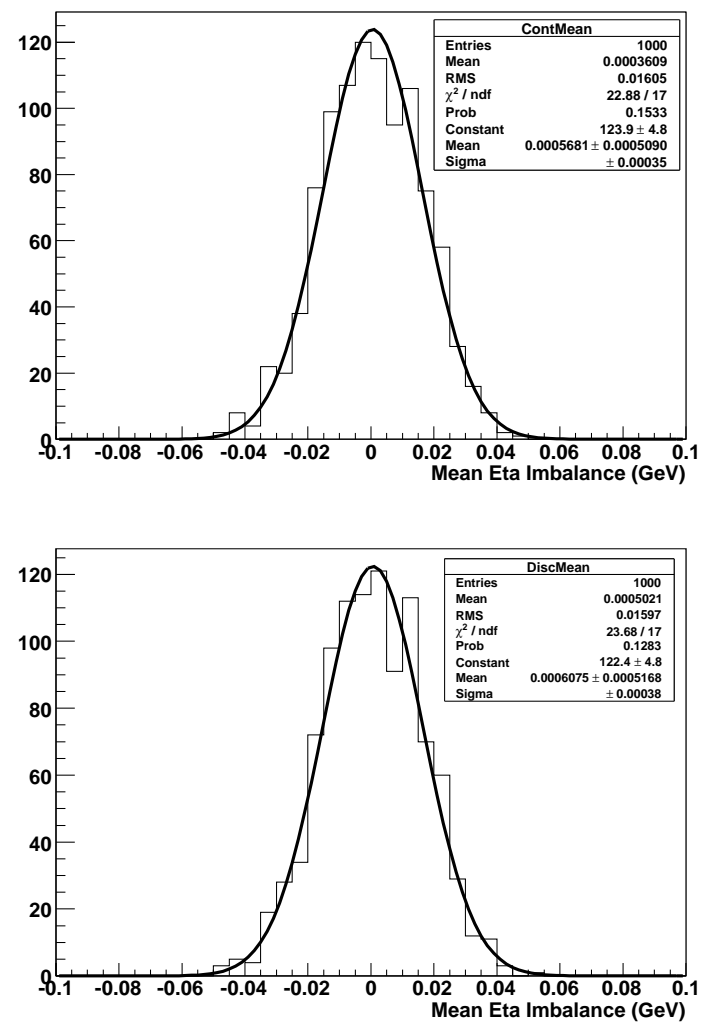

Figure A.2: Distribution of the means of $\eta$-imbalance distributions for 1000 toy $W$ sets, each generated from an independent recoil histogram containing 18k entries, with no hadronic smearing using the parameterized approach (a) and using the binned approach (b). Note how the RMSs of these two distributions are roughly equal. Since fluctuations in the mean $\eta$-imbalance represent fluctuations in the mean response for a given recoil file, the parameterized and binned approaches both model the mean response with the same precision. 
data. In the toy simulations we generate identical $p_{T}$ spectra for the $Z$ and $W$ bosons. We find, that in toy studies where the $p_{T}$ distributions are very different, the parameterized approach has a statistical advantage. Fortunately, $W$ and $Z$ boson $p_{T}$ spectra in data differ only slightly. When we model those differences in these toy studies, we find that the difference between the two approaches is negligible. It would require differences in the $p_{T}$ spectra much larger than those seen in data to even see a $10 \%$ statistical advantage in the parameterized approach.

Given the similar transverse momentum profiles of the $Z$ and $W$ bosons, the effects of bin-by-bin fluctuations in the recoil library method show up, not in the precision of the mean $\eta$-imbalance for a given toy, but in the RMS of the $\eta$-imbalance distributions. Figure A.3 shows the RMS's of the $1000 \eta$-imbalance distributions for both approaches. The average RMS of the eta imbalance for a given toy $W$ sample with recoil modeled using the binned approach is roughly 170 $\mathrm{MeV}$, whereas that of the parameterized approach is merely $17 \mathrm{MeV}$. The effect of the bin-by-bin fluctuations is to degrade the effective hadronic resolution by $\mathcal{O}(100) \mathrm{MeV}$.

So far, we have only examined the case where we do not apply any recoil resolution to the toy $W$ samples. However, the fluctuations in the toy recoil histograms also come from the hadronic resolution. If we smear the recoil system with a hadronic sampling resolution of $0.8 \sqrt{\mathrm{GeV}}$, we find that the average RMS of a typical $\eta$-imbalance distribution is around $2.137 \mathrm{GeV}$. The $\mathcal{O}(100)$ $\mathrm{MeV}$ oversmearing due to bin-by-bin fluctuations in the recoil histogram becomes insignificant when combined in quadrature with a finite resolution that is a full order of magnitude larger. Similar plot as Fig. A.2 was made with hadronic resolution turned on, and we found the RMS values of these two distributions are roughly the same as for the case with no hadronic resolution.

We can summarize as follows. In the parameterized approach, statistical fluctuations in the $Z$ recoil sample show up as fluctuations in the fitted shape of the response curve. In the binned approach, using a recoil library fluctuations in the $Z$ sample show up in two ways: as fluctuations in the mean response function over the whole look-up table, and bin-by-bin fluctuations around that mean shape, which effectively oversmear the hadronic resolution. At realistic detector resolutions, the hadronic oversmearing due to these bin-by-bin fluctuations becomes negligible, 


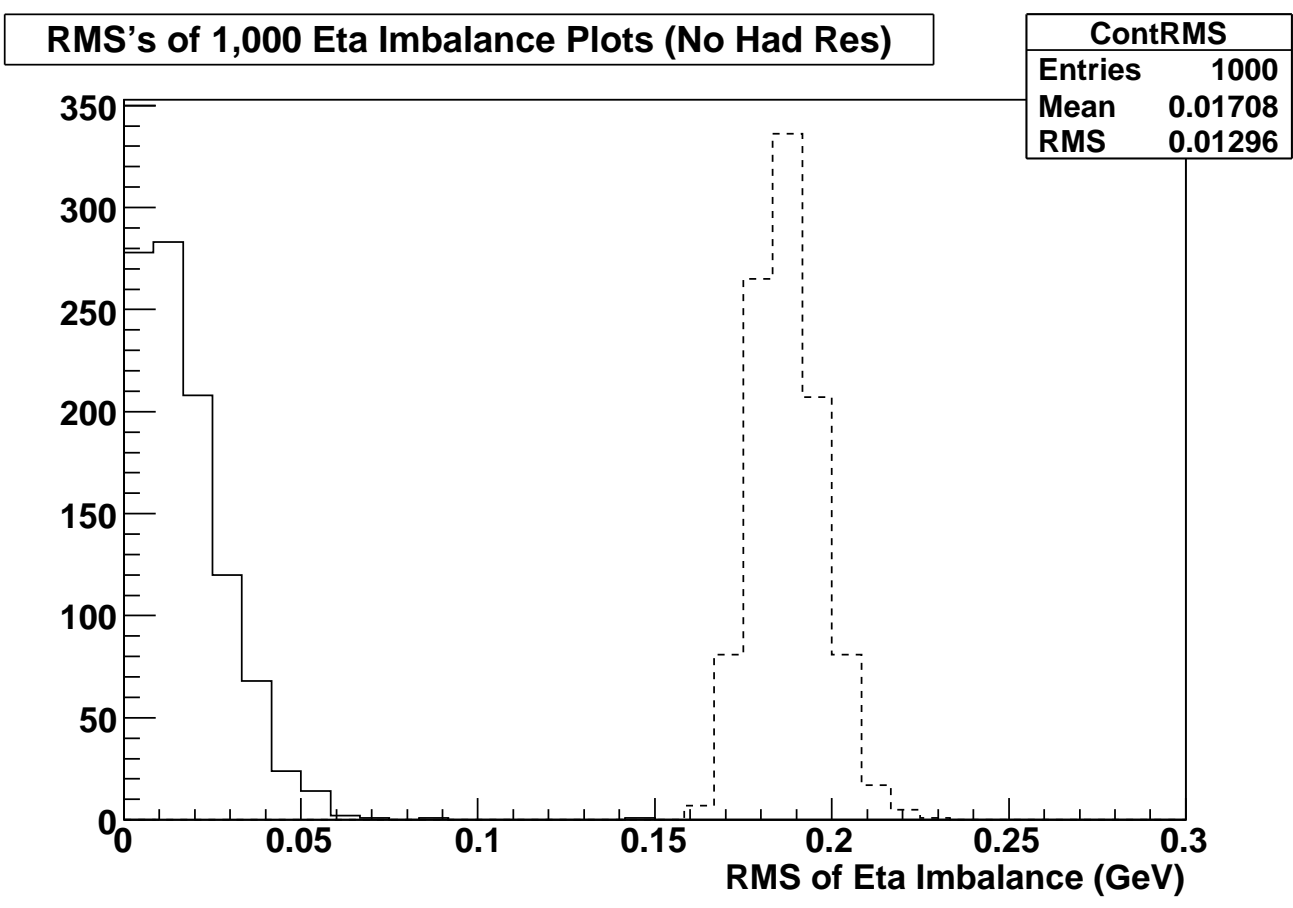

Figure A.3: The RMS's of the of $\eta$-imbalance distributions for 1000 toy $W$ sets, each generated from and independent recoil histogram containing $18 \mathrm{k}$ events, and no hadronic resolution. The solid line corresponds to the RMSs of the $\eta$-imbalance distributions using the parameterized approach. The dashed line shows the RMSs using the binned approach. Note that the average RMS of the $\eta$-imbalance distribution for the binned approach is ten times larger than that of the parameterized approach. 
whereas fluctuations in the mean recoil response remain. The statistical power of both methods to describe the mean recoil response is equivalent because the $p_{T}$ distributions are the nearly the same for both bosons. That being the case, we would expect equivalent uncertainties for both methods due to the finite $Z$ sample. This is indeed what we observe. 


\title{
Appendix B
}

$\mathrm{D} \varnothing$ Author List

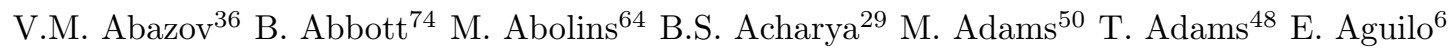

M. Ahsan ${ }^{58}$ G.D. Alexeev ${ }^{36}$ G. Alkhazov ${ }^{40}$ A. Alton ${ }^{64, a}$ G. Alverson ${ }^{62}$ G.A. Alves ${ }^{2}$ L.S. Ancu ${ }^{35}$

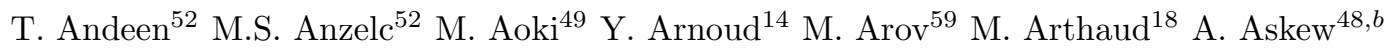

B. Åsman ${ }^{41}$ O. Atramentov ${ }^{48, b}$ C. Avila ${ }^{8}$ J. BackusMayes ${ }^{81}$ F. Badaud ${ }^{13}$ L. Bagby ${ }^{49}$ B. Baldin ${ }^{49}$

D.V. Bandurin ${ }^{58}$ P. Banerjee ${ }^{29}$ S. Banerjee ${ }^{29}$ E. Barberis ${ }^{62}$ A.-F. Barfuss ${ }^{15}$ P. Bargassa ${ }^{79}$

P. Baringer ${ }^{57}$ J. Barreto ${ }^{2}$ J.F. Bartlett ${ }^{49}$ U. Bassler ${ }^{18}$ D. Bauer ${ }^{43}$ S. Beale ${ }^{6}$ A. Bean ${ }^{57}$ M. Begalli ${ }^{3}$

M. Begel ${ }^{72}$ C. Belanger-Champagne ${ }^{41}$ L. Bellantoni ${ }^{49}$ A. Bellavance ${ }^{49}$ J.A. Benitez ${ }^{64}$ S.B. Beri ${ }^{27}$

G. Bernardi ${ }^{17}$ R. Bernhard ${ }^{23}$ I. Bertram ${ }^{42}$ M. Besançon ${ }^{18}$ R. Beuselinck ${ }^{43}$ V.A. Bezzubov ${ }^{39}$

P.C. Bhat ${ }^{49}$ V. Bhatnagar ${ }^{27}$ G. Blazey ${ }^{51}$ S. Blessing ${ }^{48}$ K. Bloom ${ }^{66}$ A. Boehnlein ${ }^{49}$ D. Boline ${ }^{61}$

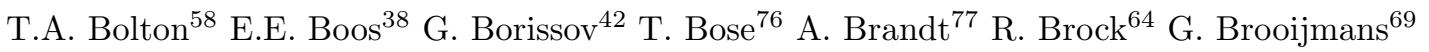

A. Bross $^{49}$ D. Brown ${ }^{19}$ X.B. Bu ${ }^{7}$ N.J. Buchanan ${ }^{48}$ D. Buchholz ${ }^{52}$ M. Buehler ${ }^{80}$ V. Buescher ${ }^{22}$

V. Bunichev ${ }^{38}$ S. Burdin ${ }^{42, c}$ T.H. Burnett ${ }^{81}$ C.P. Buszello ${ }^{43}$ P. Calfayan ${ }^{25}$ B. Calpas ${ }^{15}$

S. Calvet ${ }^{16}$ J. Cammin ${ }^{70}$ M.A. Carrasco-Lizarraga ${ }^{33}$ E. Carrera ${ }^{48}$ W. Carvalho ${ }^{3}$ B.C.K. Casey ${ }^{49}$

H. Castilla-Valdez ${ }^{33}$ S. Chakrabarti ${ }^{71}$ D. Chakraborty ${ }^{51}$ K.M. Chan ${ }^{54}$ A. Chandra ${ }^{47}$ E. Cheu ${ }^{45}$

D.K. Cho ${ }^{61}$ S. Choi ${ }^{32}$ B. Choudhary ${ }^{28}$ L. Christofek ${ }^{76}$ T. Christoudias ${ }^{43}$ S. Cihangir ${ }^{49}$ D. Claes ${ }^{66}$

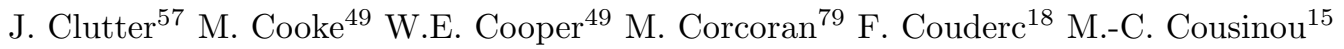

S. Crépé-Renaudin ${ }^{14}$ V. Cuplov ${ }^{58}$ D. Cutts $^{76}$ M. Ćwiok ${ }^{30}$ A. Das ${ }^{45}$ G. Davies ${ }^{43}$ K. De ${ }^{77}$

S.J. de Jong ${ }^{35}$ E. De La Cruz-Burelo ${ }^{33}$ K. DeVaughan ${ }^{66}$ F. Déliot ${ }^{18}$ M. Demarteau ${ }^{49}$

R. Demina ${ }^{70}$ D. Denisov ${ }^{49}$ S.P. Denisov ${ }^{39}$ S. Desai ${ }^{49}$ H.T. Diehl ${ }^{49}$ M. Diesburg ${ }^{49}$ A. Dominguez ${ }^{66}$

T. Dorland ${ }^{81}$ A. Dubey ${ }^{28}$ L.V. Dudko ${ }^{38}$ L. Duflot ${ }^{16}$ D. Duggan ${ }^{48}$ A. Duperrin ${ }^{15}$ S. Dutt ${ }^{27}$

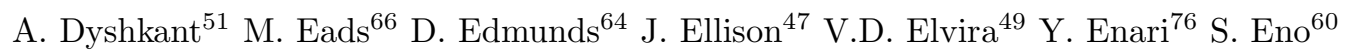

P. Ermolov ${ }^{38, \ddagger}$ M. Escalier ${ }^{15}$ H. Evans ${ }^{53}$ A. Evdokimov ${ }^{72}$ V.N. Evdokimov ${ }^{39}$ A.V. Ferapontov ${ }^{58}$

T. Ferbel ${ }^{61,70}$ F. Fiedler ${ }^{24}$ F. Filthaut ${ }^{35}$ W. Fisher ${ }^{49}$ H.E. Fisk ${ }^{49}$ M. Fortner ${ }^{51}$ H. Fox ${ }^{42}$ S. Fu ${ }^{49}$ 


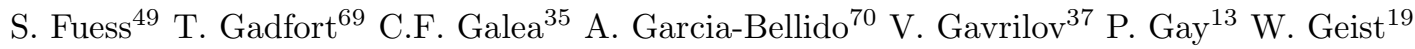

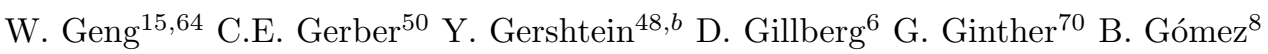

A. Goussiou ${ }^{81}$ P.D. Grannis ${ }^{71}$ S. Greder ${ }^{19}$ H. Greenlee ${ }^{49}$ Z.D. Greenwood ${ }^{59}$ E.M. Gregores ${ }^{4}$

G. Grenier ${ }^{20}$ Ph. Gris ${ }^{13}$ J.-F. Grivaz ${ }^{16}$ A. Grohsjean ${ }^{25}$ S. Grünendahl ${ }^{49}$ M.W. Grünewald ${ }^{30}$

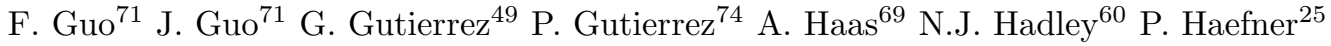

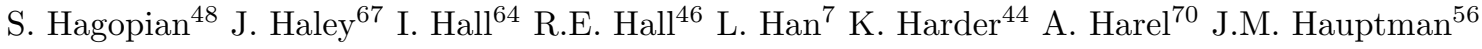
J. Hays ${ }^{43}$ T. Hebbeker ${ }^{21}$ D. Hedin ${ }^{51}$ J.G. Hegeman ${ }^{34}$ A.P. Heinson ${ }^{47}$ U. Heintz ${ }^{61}$ C. Hensel ${ }^{22, d}$

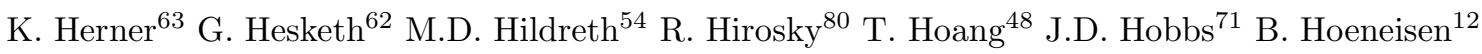

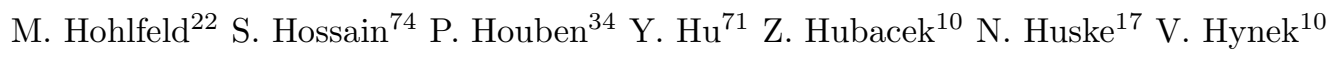

I. Iashvili ${ }^{68}$ R. Illingworth ${ }^{49}$ A.S. Ito ${ }^{49}$ S. Jabeen ${ }^{61}$ M. Jaffré ${ }^{16}$ S. Jain ${ }^{74}$ K. Jakobs ${ }^{23}$ D. Jamin ${ }^{15}$

C. Jarvis ${ }^{60}$ R. Jesik ${ }^{43}$ K. Johns ${ }^{45}$ C. Johnson ${ }^{69}$ M. Johnson ${ }^{49}$ D. Johnston ${ }^{66}$ A. Jonckheere ${ }^{49}$

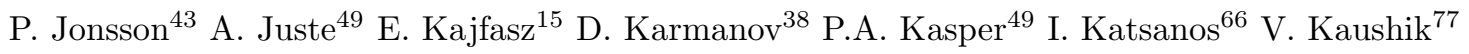

R. Kehoe ${ }^{78}$ S. Kermiche ${ }^{15}$ N. Khalatyan ${ }^{49}$ A. Khanov ${ }^{75}$ A. Kharchilava ${ }^{68}$ Y.N. Kharzheev ${ }^{36}$

D. Khatidze ${ }^{69}$ T.J. Kim ${ }^{31}$ M.H. Kirby ${ }^{52}$ M. Kirsch ${ }^{21}$ B. Klima ${ }^{49}$ J.M. Kohli ${ }^{27}$ J.-P. Konrath ${ }^{23}$

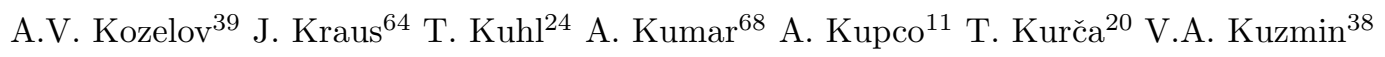

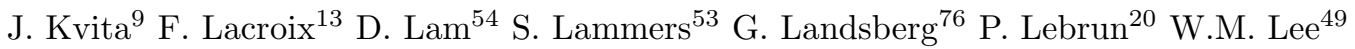

A. Leflat ${ }^{38}$ J. Lellouch ${ }^{17}$ J. Li ${ }^{77, \ddagger}$ L. Li ${ }^{47}$ Q.Z. Li $^{49}$ S.M. Lietti ${ }^{5}$ J.K. Lim ${ }^{31}$ D. Lincoln ${ }^{49}$

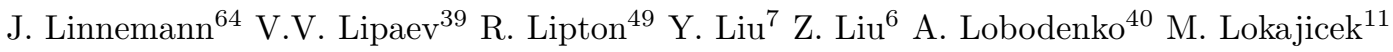

P. Love ${ }^{42}$ H.J. Lubatti ${ }^{81}$ R. Luna-Garcia ${ }^{33, e}$ A.L. Lyon ${ }^{49}$ A.K.A. Maciel ${ }^{2}$ D. Mackin ${ }^{79}$

P. Mättig ${ }^{26}$ A. Magerkurth ${ }^{63}$ P.K. Mal ${ }^{81}$ H.B. Malbouisson ${ }^{3}$ S. Malik ${ }^{66}$ V.L. Malyshev ${ }^{36}$

Y. Maravin ${ }^{58}$ B. Martin ${ }^{14}$ R. McCarthy ${ }^{71}$ C.L. McGivern ${ }^{57}$ M.M. Meijer ${ }^{35}$ A. Melnitchouk ${ }^{65}$

L. Mendoza ${ }^{8}$ P.G. Mercadante ${ }^{5}$ M. Merkin ${ }^{38}$ K.W. Merritt ${ }^{49}$ A. Meyer ${ }^{21}$ J. Meyer ${ }^{22, d}$

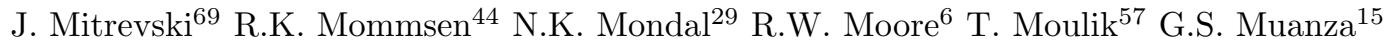

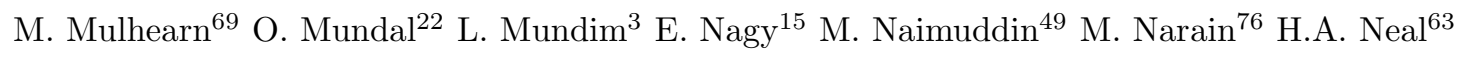

J.P. Negret ${ }^{8}$ P. Neustroev ${ }^{40}$ H. Nilsen ${ }^{23}$ H. Nogima ${ }^{3}$ S.F. Novaes ${ }^{5}$ T. Nunnemann ${ }^{25}$ D.C. O'Neil ${ }^{6}$

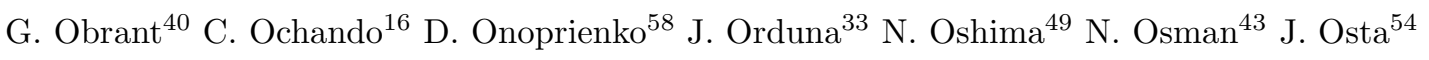

R. Otec ${ }^{10}$ G.J. Otero y Garzón ${ }^{1}$ M. Owen ${ }^{44}$ M. Padilla ${ }^{47}$ P. Padley ${ }^{79}$ M. Pangilinan ${ }^{76}$ 


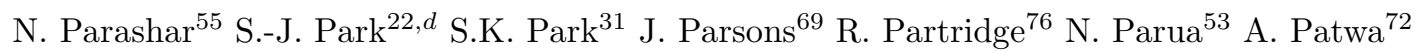

G. Pawloski ${ }^{79}$ B. Penning ${ }^{23}$ M. Perfilov ${ }^{38}$ K. Peters ${ }^{44}$ Y. Peters ${ }^{44}$ P. Pétroff ${ }^{16}$ R. Piegaia ${ }^{1}$

J. Piper ${ }^{64}$ M.-A. Pleier ${ }^{22}$ P.L.M. Podesta-Lerma ${ }^{33, f}$ V.M. Podstavkov ${ }^{49}$ Y. Pogorelov ${ }^{54}$

M.-E. Pol ${ }^{2}$ P. Polozov ${ }^{37}$ A.V. Popov ${ }^{39}$ C. Potter $^{6}$ W.L. Prado da Silva ${ }^{3}$ S. Protopopescu ${ }^{72}$

J. Qian ${ }^{63}$ A. Quadt ${ }^{22, d}$ B. Quinn ${ }^{65}$ A. Rakitine ${ }^{42}$ M.S. Rangel ${ }^{16}$ K. Ranjan ${ }^{28}$ P.N. Ratoff ${ }^{42}$

P. Renkel ${ }^{78}$ P. Rich ${ }^{44}$ M. Rijssenbeek ${ }^{71}$ I. Ripp-Baudot ${ }^{19}$ F. Rizatdinova ${ }^{75}$ S. Robinson ${ }^{43}$

R.F. Rodrigues ${ }^{3}$ M. Rominsky ${ }^{74}$ C. Royon $^{18}$ P. Rubinov ${ }^{49}$ R. Ruchti ${ }^{54}$ G. Safronov $^{37}$ G. Sajot ${ }^{14}$

A. Sánchez-Hernández ${ }^{33}$ M.P. Sanders ${ }^{17}$ B. Sanghi ${ }^{49}$ G. Savage ${ }^{49}$ L. Sawyer ${ }^{59}$ T. Scanlon ${ }^{43}$

D. Schaile ${ }^{25}$ R.D. Schamberger ${ }^{71}$ Y. Scheglov ${ }^{40}$ H. Schellman ${ }^{52}$ T. Schliephake ${ }^{26}$ S. Schlobohm ${ }^{81}$

C. Schwanenberger ${ }^{44}$ R. Schwienhorst ${ }^{64}$ J. Sekaric ${ }^{48}$ H. Severini ${ }^{74}$ E. Shabalina ${ }^{50}$ M. Shamim ${ }^{58}$

V. Shary ${ }^{18}$ A.A. Shchukin ${ }^{39}$ R.K. Shivpuri ${ }^{28}$ V. Siccardi ${ }^{19}$ V. Simak ${ }^{10}$ V. Sirotenko ${ }^{49}$ P. Skubic ${ }^{74}$

P. Slattery ${ }^{70}$ D. Smirnov ${ }^{54}$ G.R. Snow ${ }^{66}$ J. Snow ${ }^{73}$ S. Snyder ${ }^{72}$ S. Söldner-Rembold ${ }^{44}$

L. Sonnenschein ${ }^{21}$ A. Sopczak ${ }^{42}$ M. Sosebee ${ }^{77}$ K. Soustruznik ${ }^{9}$ B. Spurlock ${ }^{77}$ J. Stark ${ }^{14}$

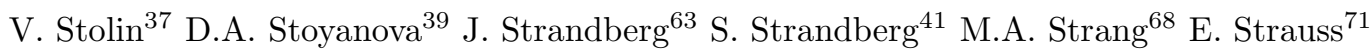

M. Strauss ${ }^{74}$ R. Ströhmer ${ }^{25}$ D. Strom ${ }^{52}$ L. Stutte $^{49}$ S. Sumowidagdo ${ }^{48}$ P. Svoisky ${ }^{35}$

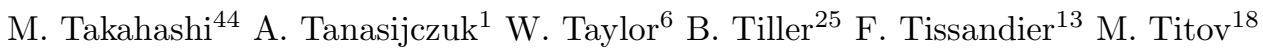

V.V. Tokmenin ${ }^{36}$ I. Torchiani ${ }^{23}$ D. Tsybychev ${ }^{71}$ B. Tuchming ${ }^{18}$ C. Tully ${ }^{67}$ P.M. Tuts $^{69}$

R. Unalan ${ }^{64}$ L. Uvarov ${ }^{40}$ S. Uvarov ${ }^{40}$ S. Uzunyan ${ }^{51}$ B. Vachon ${ }^{6}$ P.J. van den Berg ${ }^{34}$

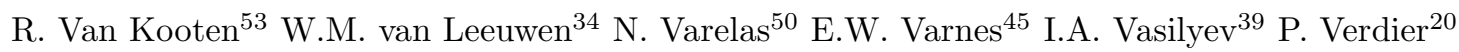

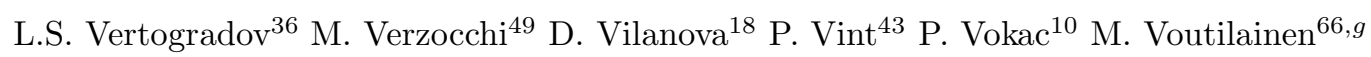

R. Wagner ${ }^{67}$ H.D. Wahl ${ }^{48}$ M.H.L.S. Wang ${ }^{49}$ J. Warchol ${ }^{54}$ G. Watts ${ }^{81}$ M. Wayne ${ }^{54}$ G. Weber ${ }^{24}$

M. Weber ${ }^{49, h}$ L. Welty-Rieger ${ }^{53}$ A. Wenger ${ }^{23, i}$ M. Wetstein ${ }^{60}$ A. White ${ }^{77}$ D. Wicke ${ }^{26}$

M.R.J. Williams ${ }^{42}$ G.W. Wilson ${ }^{57}$ S.J. Wimpenny ${ }^{47}$ M. Wobisch ${ }^{59}$ D.R. Wood ${ }^{62}$ T.R. Wyatt ${ }^{44}$

Y. Xie ${ }^{76}$ C. $\mathrm{Xu}^{63}$ S. Yacoob ${ }^{52}$ R. Yamada ${ }^{49}$ W.-C. Yang ${ }^{44}$ T. Yasuda ${ }^{49}$ Y.A. Yatsunenko ${ }^{36}$

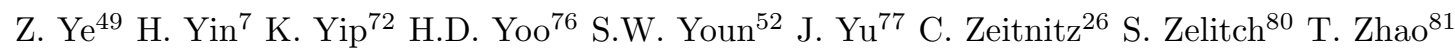

B. Zhou ${ }^{63}$ J. Zhu ${ }^{71}$ M. Zielinski ${ }^{70}$ D. Zieminska ${ }^{53}$ L. Zivkovic ${ }^{69}$ V. Zutshi ${ }^{51}$ E.G. Zverev ${ }^{38}$

(The DØ Collaboration) 
${ }^{1}$ Universidad de Buenos Aires, Buenos Aires, Argentina

${ }^{2}$ LAFEX, Centro Brasileiro de Pesquisas Físicas, Rio de Janeiro, Brazil

${ }^{3}$ Universidade do Estado do Rio de Janeiro, Rio de Janeiro, Brazil

${ }^{4}$ Universidade Federal do ABC, Santo André, Brazil

${ }^{5}$ Instituto de Física Teórica, Universidade Estadual Paulista, São Paulo, Brazil

${ }^{6}$ University of Alberta, Edmonton, Alberta, Canada; Simon Fraser University, Burnaby,

British Columbia, Canada; York University, Toronto, Ontario, Canada

and McGill University, Montreal, Quebec, Canada

${ }^{7}$ University of Science and Technology of China, Hefei, People's Republic of China

${ }^{8}$ Universidad de los Andes, Bogotá, Colombia

${ }^{9}$ Center for Particle Physics, Charles University, Faculty of Mathematics and Physics,

Prague, Czech Republic

${ }^{10}$ Czech Technical University in Prague, Prague, Czech Republic

${ }^{11}$ Center for Particle Physics, Institute of Physics, Academy of Sciences of the Czech Republic, Prague, Czech Republic

${ }^{12}$ Universidad San Francisco de Quito, Quito, Ecuador

${ }^{13}$ LPC, Université Blaise Pascal, CNRS/IN2P3, Clermont, France

${ }^{14}$ LPSC, Université Joseph Fourier Grenoble 1, CNRS/IN2P3, Institut National Polytechnique de Grenoble, Grenoble, France

${ }^{15}$ CPPM, Aix-Marseille Université, CNRS/IN2P3, Marseille, France

${ }^{16}$ LAL, Université Paris-Sud, IN2P3/CNRS, Orsay, France

${ }^{17}$ LPNHE, IN2P3/CNRS, Universités Paris VI and VII, Paris, France

${ }^{18} \mathrm{CEA}$, Irfu, SPP, Saclay, France

${ }^{19}$ IPHC, Université de Strasbourg, CNRS/IN2P3, Strasbourg, France

${ }^{20}$ IPNL, Université Lyon 1, CNRS/IN2P3, Villeurbanne, France and Université de Lyon, Lyon, France

${ }^{21}$ III. Physikalisches Institut A, RWTH Aachen University, Aachen, Germany

${ }^{22}$ Physikalisches Institut, Universität Bonn, Bonn, Germany

${ }^{23}$ Physikalisches Institut, Universität Freiburg, Freiburg, Germany

${ }^{24}$ Institut für Physik, Universität Mainz, Mainz, Germany

${ }^{25}$ Ludwig-Maximilians-Universität München, München, Germany 
${ }^{26}$ Fachbereich Physik, University of Wuppertal, Wuppertal, Germany

${ }^{27}$ Panjab University, Chandigarh, India

${ }^{28}$ Delhi University, Delhi, India

${ }^{29}$ Tata Institute of Fundamental Research, Mumbai, India

${ }^{30}$ University College Dublin, Dublin, Ireland

${ }^{31}$ Korea Detector Laboratory, Korea University, Seoul, Korea

${ }^{32}$ SungKyunKwan University, Suwon, Korea

${ }^{33}$ CINVESTAV, Mexico City, Mexico

${ }^{34}$ FOM-Institute NIKHEF and University of Amsterdam/NIKHEF, Amsterdam, The Netherlands

${ }^{35}$ Radboud University Nijmegen/NIKHEF, Nijmegen, The Netherlands

${ }^{36}$ Joint Institute for Nuclear Research, Dubna, Russia

${ }^{37}$ Institute for Theoretical and Experimental Physics, Moscow, Russia

${ }^{38}$ Moscow State University, Moscow, Russia

${ }^{39}$ Institute for High Energy Physics, Protvino, Russia

${ }^{40}$ Petersburg Nuclear Physics Institute, St. Petersburg, Russia

${ }^{41}$ Stockholm University, Stockholm, Sweden, and Uppsala University, Uppsala, Sweden

${ }^{42}$ Lancaster University, Lancaster, United Kingdom

${ }^{43}$ Imperial College, London, United Kingdom

${ }^{44}$ University of Manchester, Manchester, United Kingdom

${ }^{45}$ University of Arizona, Tucson, Arizona 85721, USA

${ }^{46}$ California State University, Fresno, California 93740, USA

${ }^{47}$ University of California, Riverside, California 92521, USA

${ }^{48}$ Florida State University, Tallahassee, Florida 32306, USA

${ }^{49}$ Fermi National Accelerator Laboratory, Batavia, Illinois 60510, USA

${ }^{50}$ University of Illinois at Chicago, Chicago, Illinois 60607, USA

${ }^{51}$ Northern Illinois University, DeKalb, Illinois 60115, USA

${ }^{52}$ Northwestern University, Evanston, Illinois 60208, USA

${ }^{53}$ Indiana University, Bloomington, Indiana 47405, USA

${ }^{54}$ University of Notre Dame, Notre Dame, Indiana 46556, USA

${ }^{55}$ Purdue University Calumet, Hammond, Indiana 46323, USA 
${ }^{56}$ Iowa State University, Ames, Iowa 50011, USA

${ }^{57}$ University of Kansas, Lawrence, Kansas 66045, USA

${ }^{58}$ Kansas State University, Manhattan, Kansas 66506, USA

${ }^{59}$ Louisiana Tech University, Ruston, Louisiana 71272, USA

${ }^{60}$ University of Maryland, College Park, Maryland 20742, USA

${ }^{61}$ Boston University, Boston, Massachusetts 02215, USA

${ }^{62}$ Northeastern University, Boston, Massachusetts 02115, USA

${ }^{63}$ University of Michigan, Ann Arbor, Michigan 48109, USA

${ }^{64}$ Michigan State University, East Lansing, Michigan 48824, USA

${ }^{65}$ University of Mississippi, University, Mississippi 38677, USA

${ }^{66}$ University of Nebraska, Lincoln, Nebraska 68588, USA

${ }^{67}$ Princeton University, Princeton, New Jersey 08544, USA

${ }^{68}$ State University of New York, Buffalo, New York 14260, USA

${ }^{69}$ Columbia University, New York, New York 10027, USA

${ }^{70}$ University of Rochester, Rochester, New York 14627, USA

${ }^{71}$ State University of New York, Stony Brook, New York 11794, USA

${ }^{72}$ Brookhaven National Laboratory, Upton, New York 11973, USA

${ }^{73}$ Langston University, Langston, Oklahoma 73050, USA

${ }^{74}$ University of Oklahoma, Norman, Oklahoma 73019, USA

${ }^{75}$ Oklahoma State University, Stillwater, Oklahoma 74078, USA

${ }^{76}$ Brown University, Providence, Rhode Island 02912, USA

${ }^{77}$ University of Texas, Arlington, Texas 76019, USA

${ }^{78}$ Southern Methodist University, Dallas, Texas 75275, USA

${ }^{79}$ Rice University, Houston, Texas 77005, USA

${ }^{80}$ University of Virginia, Charlottesville, Virginia 22901, USA

${ }^{81}$ University of Washington, Seattle, Washington 98195, USA 


\section{BIBLIOGRAPHY}

[1] D.P. Saltzberg A Measurement of the W Mass, CDF PhD Thesis, Enrico Fermi Institute, University of Chicago (1994).

[2] D. Griffiths, Introduction to Elementary Particles, Heppenheim, Germany: Wiley-VCH, (2004).

[3] I. Aitchison, A. Hey, Gauge Theories in Particle Physics, Vols. 1 and 2, Philadelphia: IOP Publishing (2004)

[4] D. Perkins, Introduction to High Energy Physics.

[5] P. Higgs, Broken Symmetries and the Masses of Gauge Bosons. Phys. Rev. Lett. 13508 (1964)

[6] L.H. Ryder, Quantum Field Theory, Cambridge University Press (1996)

[7] A. Zee, Quantum Field Theory In a Nutshell, Princeton University Press (2003)

[8] S.L. Glashow, Nucl. Phys. B22 579 (1961);

A. Salam and J.C. Ward, Phys. Rev. Lett. 13168 (1994);

S. Weinberg, Phys. Rev. Lett. 191264 (1967).

[9] G. Arnison et al. (UA1 Collaboration), Phys. Lett. B122 102 (1983);

M. Banner et al. (UA2 Collaboration), Phys. Lett. B122 476 (1983).

[10] CERN-PPE/95-172, LEP Electroweak Working Group (1995).

[11] W. M. Yao et al., Review of Particle Physics J. Phys. G 33, 1 (2006)

[12] L. Wang, Measurement of $Z$ Boson Momentum in Proton-Antiproton Collisions at $\sqrt{s}=1.96$ $T e V$, D $\varnothing$ Ph.D. Thesis, University of Maryland (2007).

[13] P. Nadolsky, Theory of $W$ and $Z$ Boson Production, AIP Conf. Proc., 753, 158, (2005).

[14] UA5 Collaboration, Charged Particle Multiplicity Distributions at 200 and 900 GeV C.M. Energy, Zeitschrift fur Physik C Particles and Fields, Volume 43, Issue 3, pp.357-374 (1987).

[15] J. Collins, D. Soper, and G. Sterman, Nucl. Phys. B250, 199 (1985);

J. Collins and D. Soper, Nucl. Phys. B193, 381 (1981); Nucl. Phys. B197, 446 (1982); Nucl. Phys. B213, 545(E) (1983).

[16] W. J. Marciano, Phys. Rev. D20, 274 (1979);

F. Antonelli, M. Consoli, and G. Corbo, Phys. Lett. B 91, 90(1980);

M. Veltman, Phys. Lett. B 91, 95 (1980);

[17] The LEP Electroweak Working Group, R. Strőhmer, Review of the Properties of the $W$ Boson at LEP, and the Precision Determination of its Mass, hep-ph/0401146.

[18] D. Green, Lectures in Particle Physics;

[19] K. Hagiwara et al., Phys. Rev. D66 010001 (2002).

[20] R. Rosner, Phys. Rev. D49, 1363 (1994))

[21] B. Abbott et al. (DØ Collaboration), Phys. Rev. Lett. 80 (1998);

B. Abbott et al. (DØ Collaboration), Phys. Rev. D58 (1998);

B. Abbott et al. (DØ Collaboration), Phys. Rev. Lett. 84 (2000);

B. Abbott et al. (DØ Collaboration), Phys. Rev. D62 (2000);

V. Abazov et al. (DØ Collaboration), Phys. Rev. D66 (2002). 
[22] T. Affolder et al. (CDF Collaboration), Phys. Rev. D 64, 052001 (2001).

[23] T. Aaltonen et al. (CDF Collaboration), Phys. Rev. Lett. 99, 151801 (2007);// T. Aaltonen et al. (CDF Collaboration), Phys. Rev. D 77, 112001 (2008).

[24] Tim Andeen, Measurement of the W Boson Mass with the DØ Run II Detector Using the Electron Et Spectrum Ph.D. thesis, Northwestern University, (2008).

[25] Ian Adam, Measurement of the W Boson Mass with the DØ Detector Using the Electron Et Spectrum, Ph.D. thesis, Columbia University, (1997).

[26] T. Affolder et al. (CDF Collaboration), Phys. Rev. Lett. 85, 3347 (2000).

[27] J. Zhu, Direct Measurement of the W Boson Decay Width in Proton-Anitproton Collisions at sqrts $=1.96 \mathrm{TeV}, \mathrm{D} \varnothing \mathrm{PhD}$ Thesis, University of Maryland (2004).

[28] V. M. Abozov et al. (DØ Collboration), Phys. Rev. D66, 032008 (2002).

[29] C. Balazs and C.P. Yuan, Phys.Rev. D56 5558-5583

[30] E. Barbiero and Z. Was, PHOTOS: A Universal Monte Carlo for QED radiative corrections. Version 2.0 Comput. Phys. Commun 79,291 (1994)

[31] T. Sjöstrand, L. Lőnnblad, S. Mrenna and P.

[32] Monte Carlo Generators for W Mass Analysis, J. Zhu, D0 Note xxxx August 29, 2005

[33] Application Software Group, GEANT: Detector Description and Simulation Tool, CERN Program Library Long Writeup W5013, http://wwwasd.web.cern.ch/wwwasd/geant/.

[34] B. P. Flannery et al., Numerical Recipes in C, Cambridge University Press(1992), http://www. numerical-recipes.com/.

[35] M. Wetstein, Fitting Package for W Mass Measurement, DØ Note 5663 (2007).

[36] F. James and M. Roos, Minuit: A System For Function Minimization And Analysis Of The Parameter Errors And Correlations, Comput. Phys. Commun. 10, 343 (1975).

[37] J. Stark and P. Pétroff, DØ Note 5388, 2007.

[38] T. Andeen, M. Cwiok, S. Eno, M. Grunewald, F. Guo, J. Guo, M. Hildreth, J. Hobbs, R. McCarthy, A. Meniltchouk, J. Osta, P. Petroff, M. Rijssenbeek, H. Schellman, J. Stark, M. Wetstein, S. Yacoob and J. Zhu Measurement of the $W$ boson mass using RunIIa Data DØ Note 5868

[39] J. Thompson, Introduction to Colliding Beams at Fermilab, FERMILAB-TM-1909 (1994); Run II Handbook, http://www-bd.fnal.gov/runII/; Accelerator Concepts, V3.0 (2002).

[40] DØ Collaboration, S. Abachi et al., The DØ Detector, Nucl. Instrum. Meth. A338, 185 (1994).

[41] DØ Collaboration, V.M. Abazov et al., The Upgraded DØ Detector, hep-physics/0507191, submitted to Nucl. Instrum. Meth. A (2005).

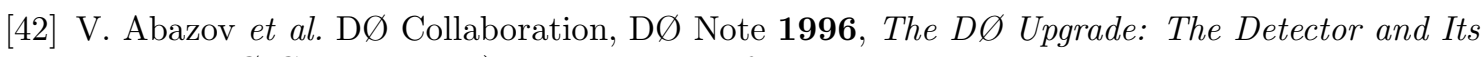
Physics; DØ Collaboration), in preparation for submission to Nucl. Instr. and Methods.

[43] DØ Upgrade Collaboration, DØ Silicon Tracker Technical Design Report, http://wwwd0.fnal.gov/trigger/stt/smt/smt_tdr.ps.

[44] DØ Collaboration, Central Fiber Tracker Technical Design Report, DØ Note 4164. 


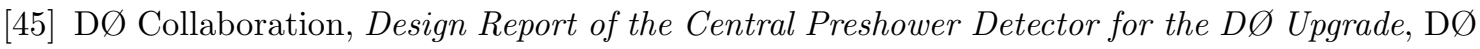
Note 3014 .

[46] DØ Collaboration, The DØ Upgrade: Forward Preshower, Muon System and Level 2 Trigger, DØ Note 2894.

[47] D. Alton and J. Konrath Energy Measurements With the Central Preshower Detector, DØ Note 4665 (2004).

[48] S. Abachi et al. (DØ Collaboration), Nucl. Instr. and Methods, A324, 53 (1993).

[49] R. Wigmans, EM Calorimetry - Energy measurements in particle physics, Oxford Science Publications, Clarendon Press, Oxford (2000).

[50] K.M. Chan, D.K. Cho, M. Zielinski, V.V. Zutshi New Sets of Calorimeter Layer Weights for preco04 and p05, dzero Note 3835 (2001).

[51] K.M. Chan, D.K. Cho, M. Zielinski, V.V. Zutshi Electron and Photon Energy Resolution in the Central Calorimeter for Run 2, dzero Note 3535 (1998).

[52] Calorimeter electronics upgrade for Run II, technical design report version 1.7, http://www- d0.fnal.gov/hardware/cal/intro/tdr/tdrformat.htm (1998).

[53] DØ Collaboration, Calorimeter Electronics, http://www-d0.fnal.gov/hardware/cal.

[54] A. Kumar, K. Ranjan and R. Zitoun, Calibration pulser system: linearity ramp studies, presentation at the calorimeter operations meeting on Nov. $6^{\text {th }}, 2003$.

[55] J. Stark, Performance of the p17 electronics calibration, DØ note in preparation, and presentation at the Calorimeter Operations meeting on Feb. $17^{\text {th }}, 2005$.

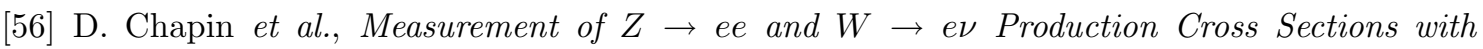
$|\eta|<2.3$.

[57] J. Stark and M. Verzocchi, Parasitic data-taking for phi intercalibration after the shutdown, presentation at the CALGO meeting on Sept. $20^{t h}, 2004$.

[58] Paul Grannis Calibrations Using Collider Events, DØ Note 796 (1989).

[59] Qiang Zhu Measurement of the W Boson Mass in Proton-Antiproton Collisions at sqrt(s)=1.8 $T e V \mathrm{D} \varnothing \mathrm{PhD}$ Thesis, New York University (1994).

[60] P.A. Delsart, J. Donini, P. Lebrun Phi-intercalibration of the D0 calorimeter at Run II, DØ Note 4299 (2003).

[61] M. Wetstein, J. Stark, M. Verzocchi Gain Calibration for the EM Calorimeter in Run II D Note 5004 .

[62] J.A. Nelder, R. Mead A Simplex Method for Function Minimization, Computer Journal 7308 (1965).

[63] G. Grindhammer, M. Rudowicz, and S. Peters The Fast Simulation of Electromagnetic and Hadronic Showers, Nucl. Instrum. Meth. A 290, 469 (1990).

[64] J. Stark, The calorimeter, dead material, and a precise simulation thereof, presentation at the All DØMeeting on June $9^{\text {th }}, 2006$.

Slides are available on the Agenda Server.

[65] T.C. Heuring, Ph.D. thesis, State University of New York at Stony Brook, 1993, Electrons in the DØ Calorimeter: A Study of the Systematic Biases in the Measurement of the W Mass (unpublished). 
[66] M. Wetstein Determination of the Sampling Resolution for the CC Region of the EM Calorimeter in Run II DØ Note 5512.

[67] DØ Integrated Luminosity: http://www-d0.fnal.gov/runcoor/runplans/runplan.html\#plots; Tevatron Integrated Luminosity: http://www-d0.fnal.gov/runcoor/RUN/run2_lumi.html.

[68] http://www-d0.fnal.gov/computing/algorithms/calgo/jet_met/runsel.html.

[69] Jet Met Group, Run Selection version 3.0, http://www-d0.fnal.gov/d0upgrad/d0_private/software/jetid/jetid-certif.html.

[70] DØ Collaboration, DØ Run II Level 1 Trigger Framework Technical Design Report, http://www.pa.msu.edu/hep/d0/ftp/11/framework/11fw_tdr_05june98.txt.

[71] D. Edmunds et al, Technical Design Report for the Level 2 Global Processor, DØ Note 3402.

[72] DØ Collaboration, Description of DØ L3 Trigger Software, DØ Note 3266.

[73] M. Adams et. al., Level 2 Calorimeter Preprocessor Technical Design Report, DØ Note 3651.

[74] D. Adams, DØ Note 2958, Finding Tracks, DØ Note 4403.

[75] H. Greenlee, DØ Note 4303, The DØ Kalman Track Fit. http://www-d0.fnal.gov/computing/algorithms/\#intro.

[76] A. Khanov, DØ Note 3778, HTF: histogramming method for finding tracks: The algorithm description.

G. Hesketh, DØ Note 4079, Central Track Extrapolation Through the DØ Detector.

H. Greenlee, DØ Note 4180, Motion of a Charged Particle in a Magnetic Field.

H. Greenlee, DØ Note 4293, The DØ Interacting Propagator.

[77] A. Schwartzman and M. Narain, Primary Vertex Selection, DØ Note 3907.

[78] Tim Andeen and Alex Melnitchouk, D0 Note 5662 http://www-d0.hef.kun.nl/fullAgenda.php?ida=a081142 electron energy response fit

[79] Determination of the constant term of the electron resolution, A. Melnitchouk, M. Wetstein, DØ Note 5879, Feb. 2009.

[80] F. Guo, J. Hobbs, R. Mccarthy, R. Rijssenbeek, J. Zhu, P. Petroff, and J. Stark Study of Energies Below Electron Window for W Mass Measurement D $\varnothing$ Note 5661 (2008).

[81] F. Guo, J. Hobbs, R. Mccarthy, R. Rijssenbeek, J. Zhu, P. Petroff, and J. Stark Study of Zero Suppression and Cluster Window Size on the Energy Measurement of Single Electrons $\mathrm{D} \emptyset$ Not e $\mathbf{5 6 6 0}$ (2008).

[82] Studies of EM PhiMod efficiency and shift for the $W$ mass measurement J. Hobbs, J. Osta, H. Schellman, J. Stark, J. Zhu DØ Note 5914.

[83] H. Schellman Run IIb Longitudinal Beam Shape, DØ Note 5547 (2007).

[84] J. Hays, J. Mitrevski, C. Schwanenberger, T. Toole, D0 note 5105.

[85] T. Andeen, F. Guo, J. Guo, and J. Zhu, Effect of Recoil on Electron Identification Efficiency, DØ Note 5686 (2008)

[86] V. Abazov et al. (DØ Collaboration), A Novel Method for Modelling the Recoil in W Events at Hadron Colliders, Nucl. Inst. and Meth., paper in review.

[87] G. D'Agostini A Multidimensional Unfolding Method Based on Bayes' Theorem Nucl. Instrum. and Meth. A 362, 487-498. (1995) 
[88] J. Osta, M. Cwiok, J. Zhu, J. Stark, and T. Andeen Modelling the Hadronic Recoil in the W Mass Measurement at Run II DØ Note 5668 (2008).

[89] J. Guo, J. Zhu, J. Hobbs and R. Mccarthy, D0 note 5665.

Full MC Note

[90] T. Andeen et al, $W$ mass measurement in Full Geant Monte Carlo, DØ Note 5659.

[91] PDF Uncertainty for W Mass Measurement, J. Guo, J. Zhu, J. Hobbs and R. Mccarthy, D0 Note nnnn April 3, 2006

[92] F. Landry, R.Brock, P. Nadosky, C.P. Yuan, Tevatron Run-1 Z boson data and Collins-SoperSterman resummation formalism, Physical review D, 67, 073016, 2003 